\title{
Molecular Recognition and Co-Crystallization of Methylated and Halogenated Fragments of Danicalipin A by Enantiopure Alleno- Acetylenic Cage Receptors
}

\author{
Supporting Information \\ Cornelius Gropp ${ }^{\dagger}$, Stefan Fischer ${ }^{\dagger}$, Tamara Husch ${ }^{\ddagger}$, Nils Trapp ${ }^{\dagger}$, Erick M. Carreira*, ${ }^{*}$ and \\ François Diederich*,†
}




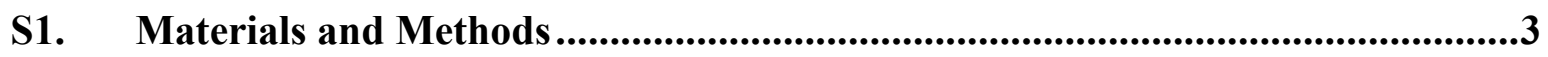

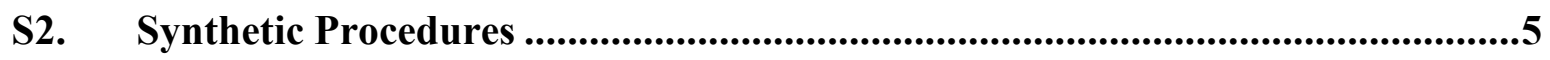

S3. Methods for Solution Binding Studies....................................................13

S4. Titration Data and Binding Isotherms Obtained by ECD Spectroscopic

Titrations ...................................................................................................14

S5. Titration Data and Binding Isotherms Obtained by ITC..............................28

S6. Summary of Association Constants Obtained from ECD Spectroscopic and

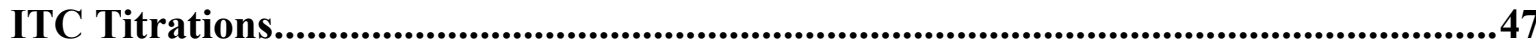

S7. Comparison of the Gibbs Binding Energies for Selected Methyl- and Bromo-

Containing Alcohols .........................................................................................49

S8. NMR Spectra of $(P)_{4}$-Configured AAC with Guest Molecules.........................49

S9. Splitting of the Host-OH-Bonding Array upon Complexation of Guests .........84

S10. J-Based Conformational Analysis of Guests 7 and 8 in Acetonitrile ................84

S11. Structures Obtained from Single Crystal X-ray Diffraction.............................89

S12. NMR Spectra of $(P)_{4}$-Configured AAC and Guest Compounds ....................107

Theoretical Section ................................................................................120

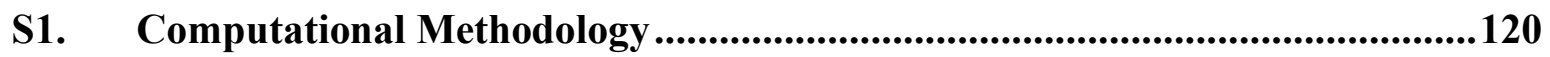

S2. Conformational Analysis ...........................................................................120

S3. Effect of Computational Methodology ...............................................................123

S4. Determination of Packing Coefficients.........................................................125

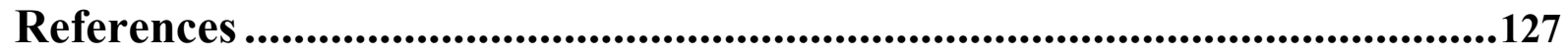




\section{Experimental Section}

\section{S1. Materials and Methods}

Chemicals: Chemicals for guest binding studies and for crystallization experiments were used without prior purification (ABCR, Acros, Sigma Aldrich, Fluka, TCI). AACs $(P)_{4-}$ and $(M)_{4-}$ 1 were synthesized following the reported procedure. ${ }^{[1]}$ Corresponding ${ }^{1} \mathrm{H},{ }^{13} \mathrm{C}$, and ${ }^{19} \mathrm{~F}$ NMR spectra are attached for $\mathrm{AAC}(P)_{4}-\mathbf{1}$ and the synthesized guest molecules. All compounds were purchased in their racemic form or in their enantiomerically pure form, where indicated.

${ }^{1}$ H NMR spectra were recorded on a Gemini $400\left(400 \mathrm{MHz},{ }^{1} \mathrm{H}\right)$ instrument at $298 \mathrm{~K}$. Chemical shifts $\left(\delta_{\mathrm{H}}\right)$ are quoted in parts per million $(\mathrm{ppm})$, referenced to the residual solvent peak $\left(\mathrm{CDCl}_{3}\right.$, $\delta_{\mathrm{H}}$ 7.226). Coupling constants $(J)$ are reported to the nearest $0.5 \mathrm{~Hz}$. Multiplicities are reported as follows: $\mathrm{s}=$ singlet, $\mathrm{d}=$ doublet, $\mathrm{t}=$ triplet, $\mathrm{q}=$ quartet, $\mathrm{m}=$ multiplet, or as a combination of them. Coupling constants $J$ are given in $\operatorname{Hertz}(\mathrm{Hz})$.

${ }^{13}$ C NMR spectra were recorded on a Bruker DRX $400\left(100 \mathrm{MHz},{ }^{13} \mathrm{C}\right)$ spectrometer. Chemical shifts $\left(\delta_{\mathrm{C}}\right)$ are quoted in ppm referenced to the appropriate solvent peak $\left(\mathrm{CDCl}_{3}, \delta 77.0 \mathrm{ppm}\right)$.

ID and 2D NMR spectra of the host-guest complexes were recorded on a Bruker AVIII 600 Ultra Shell spectrometer equipped with a Prodigy Cryo Probe $\left(600 \mathrm{MHz}\right.$ for ${ }^{1} \mathrm{H} ; 150 \mathrm{MHz}$ for $\left.{ }^{13} \mathrm{C}\right)$.

Isothermal Titration Calorimetry (ITC) was performed on a MicroCal VP-ITC calorimeter. 25 Portions of $10 \mu \mathrm{L}$ "guest"-solution were added to a "host"-solution at intervals of $240 \mathrm{~s}$. The power $(P)$ that was consumed to keep the sample temperature at $303 \mathrm{~K}$ was monitored. The heat of dilution of "guest"-solution added to pure solvent (n-octane) was measured and subtracted. The MicroCal Origin 5.0 software was used for data treatment. ${ }^{[2]}$

Electronic Circular Dichroism (ECD) spectra were recorded on a JASCO Corp. J-715, Rev. 1.00 instrument. The spectra were measured in a quartz cuvette $(1 \mathrm{~cm})$ at $293 \mathrm{~K}$. The absorption wavelength is reported in $\mathrm{nm}$ with the molar extinction coefficient $\Delta \varepsilon\left(\mathrm{dm}^{3} \mathrm{~mol}^{-1}\right.$ $\left.\mathrm{cm}^{-1}\right)$.

Infrared spectra (IR) were recorded on a Perkin-Elmer 1600 FT-IR spectrometer (ATR, Golden State). Only selected absorbances $\left(v_{\max }\right)$ are reported. The samples are reported as absorption 
maxima in $\mathrm{cm}^{-1}$ with corresponding relative intensities described as sh (shoulder), $\mathrm{s}$ (strong), m (medium), and w (weak).

Optical Rotation was recorded on a Perkin-Elmer 1600 FT-IR spectrometer 241 polarimeter.

High Resolution Mass spectra (HR-MS) measurements were performed by the MS service at the Laboratory of Organic Chemistry of ETH Zurich. EI-MS: Waters Micromass AutoSpeUltima spectrometer; ESI-MS: Bruker maXis spectrometer. HR-MALDI: Varian IonSpec FT ICR; Masses are reported in $m / z$ units as the molecule ion $\mathrm{M}+,[M+\mathrm{H}]^{+},[M+\mathrm{Na}]^{+},[M+\mathrm{K}]^{+}$, with the corresponding intensities in $\%$.

X-ray Intensity Data were measured on a Rigaku XtaLAB Synergy diffractometer equipped with a Dectris Pilatus $300 \mathrm{~K}$ hybrid pixel array detector, using microfocus sealed-tube $\mathrm{Cu}-\mathrm{K \alpha}$ radiation with mirror optics $(\lambda=1.54184 \AA)$ at the given temperature. Samples were mounted on MiTeGen Micromount Kapton sample holders in perfluoroalkylether oil. Collected data were processed with the CrysAlisPro ${ }^{[3]}$ software package and corrected for absorption effects using the multi-scan or Gaussian method, or a combination thereof. Structures were solved and refined using the OLEX2 $2^{[4]}$ and SHELX ${ }^{[5]}$ software packages. Generally, wherever relative occupancies (in \%) within disordered regions are discussed, they are directly taken from the refined free variables. The authors are aware that this method can be rather unreliable due to a variety of reasons and hence for all purposes of discussion we assumed an error margin of $+/-$ 5\%. Additionally, care was taken not to over-restrain disorder of the guest molecules. Only soft similar distance restraints (SADI) and enhanced rigid bond restraints (RIGU) were applied in this region. Their usage was kept at the practicable minimum.

Supplementary data for the obtained X-ray co-crystal structures (CCDC 1914836-1914843) can be obtained free of charge from The Cambridge Crystallographic Data Centre, 12 Union Road, Cambridge CB2 1EZ, UK (fax: +44(1223)-336-033; e-mail: deposit@ccdc.cam.ac.uk), or online via https://www.ccdc.cam.ac.uk/getstructures. HKL data and refinement instructions, as well as applied restraints, are included with the deposited files. 


\section{S2. Synthetic Procedures}<smiles>CCCCO</smiles>

2<smiles>CC(C)=C([15NH])CO</smiles><smiles>C[C@@H](Br)[C@H](Br)CO</smiles>

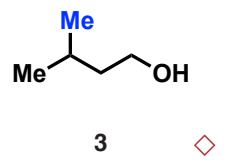<smiles>C[C@H](Cl)[C@@H](CO)[Se-][TeH]</smiles><smiles>CC(C)C(C)(C)CO</smiles>

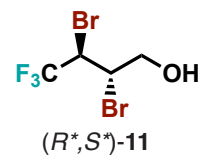

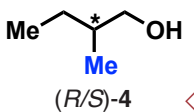

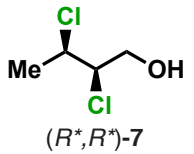<smiles>CC(Br)[C@](Br)(CO)[C@@](O)(Br)CO</smiles><smiles>[N+][C@](Br)(CO)[C@@H](Br)C(F)(F)F</smiles>

$\left(R^{*}, S^{*}\right)-12$

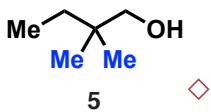<smiles>C[C@@H](Br)[C@H](Br)CO</smiles><smiles>CC(Br)C(Br)(CO)CO</smiles>

Figure S1. Guest molecules selected for complexation studies with enantiopure AACs $(P)_{4-1}$ and $(M)_{4}-1$. Structures designated with a red square $(\diamond)$ are commercially available. All other molecules were prepared in a racemic fashion following the procedures below.

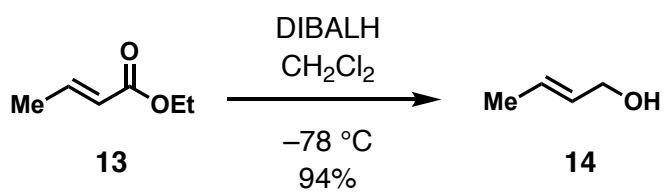

(E)-But-2-en-1-ol (14). Ethyl enoate 13 (5.1 mL, 48 mmol, 1.0 equiv) was dissolved in $\mathrm{CH}_{2} \mathrm{Cl}_{2}$ (43 mL) and cooled to $0{ }^{\circ} \mathrm{C}$. DIBALH ( $1 \mathrm{M}$ in $\mathrm{CH}_{2} \mathrm{Cl}_{2}, 100 \mathrm{~mL}, 100 \mathrm{mmol}, 2.1$ equiv.) was added, and the mixture was stirred at $0{ }^{\circ} \mathrm{C}$ for $1 \mathrm{~h}$. The mixture was quenched with the addition of $\mathrm{MeOH}(5 \mathrm{~mL})$ at $0{ }^{\circ} \mathrm{C}$ and allowed to reach ambient temperature. After the addition of sat. aq. $\mathrm{NaCl}$ solution $(50 \mathrm{~mL})$ and $\mathrm{Et}_{2} \mathrm{O}(50 \mathrm{~mL})$, the mixture was filtered through a plug of Celite. The layers were separated, dried over $\mathrm{Na}_{2} \mathrm{SO}_{4}$ and concentrated under reduced pressure. Purification by flash column chromatography $\left(\mathrm{SiO}_{2}, \mathrm{Et} 2 \mathrm{O} /\right.$ pentane 1:1) afforded allylic alcohol 3 (3.2 g, $45 \mathrm{mmol}, 94 \%)$ as a colorless liquid.

Reference spectrum: Chem. Eur. J. 2015, 21, 7408-7412.

${ }^{1} \underline{\mathrm{H} N M R}\left(400 \mathrm{MHz}, \mathrm{CDCl}_{3}\right): \delta 5.81-5.58(\mathrm{~m}, 2 \mathrm{H}), 4.13-4.00(\mathrm{~m}, 2 \mathrm{H}), 1.71(\mathrm{dt}, J=4.7,1.1 \mathrm{~Hz}$, 3H) $\mathrm{ppm}$. 


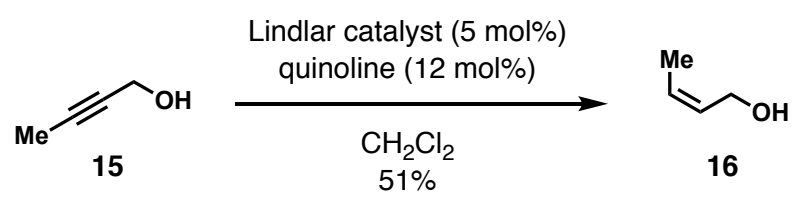

(Z)-But-2-en-1-ol (16). A mixture of alkyne 15 (2.9 g, $41 \mathrm{mmol}, 1.0$ equiv), quinoline (0.58 mL, $4.9 \mathrm{mmol}, 0.12$ equiv), and LindLAR catalyst (5 wt\% $\mathrm{Pd}, 4.3 \mathrm{~g}, 2.0 \mathrm{mmol}$, 0.05 equiv) in $\mathrm{CH}_{2} \mathrm{Cl}_{2}(50 \mathrm{~mL})$ was stirred under a $\mathrm{H}_{2}$ atmosphere for 1 week. The mixture was filtered through a plug of Celite and then concentrated under reduced pressure. Distillation $\left(110^{\circ} \mathrm{C}, 1 \mathrm{~atm}\right)$ afforded olefin $16(1.5 \mathrm{~g}, 21 \mathrm{mmol}, 51 \%)$ as a colorless liquid.

Reference spectrum: Chem. Commun. 2013, 49, 7037-7039.

${ }^{1} \underline{\mathrm{H} \mathrm{NMR}}\left(400 \mathrm{MHz}, \mathrm{CDCl}_{3}\right): \delta 5.68-5.57(\mathrm{~m}, 2 \mathrm{H}), 4.26-4.17(\mathrm{~m}, 2 \mathrm{H}), 1.73-1.61(\mathrm{~m}, 3 \mathrm{H}) \mathrm{ppm}$.

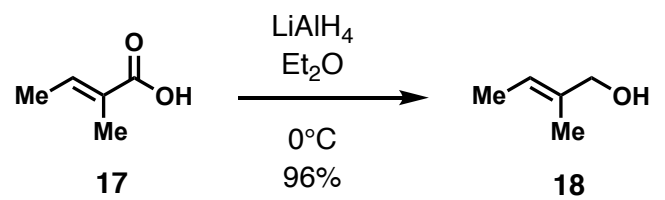

(E)-2-Methylbut-2-en-1-ol (18). Tiglic acid $17(5.0 \mathrm{~g}, 50 \mathrm{mmol}, 1.0$ equiv) was added dropwise to a suspension of $\mathrm{LiAlH}_{4}\left(3.8 \mathrm{~g}, 100 \mathrm{mmol}, 2.0\right.$ equiv) in $\mathrm{Et}_{2} \mathrm{O}(20 \mathrm{~mL})$ at $0{ }^{\circ} \mathrm{C}$. The suspension was allowed to reach ambient temperature over $16 \mathrm{~h}$. After slow addition of $\mathrm{NaOH}$ $(3 \mathrm{M}, 20 \mathrm{~mL})$ at $0{ }^{\circ} \mathrm{C}$ and $\mathrm{Et}_{2} \mathrm{O}(100 \mathrm{~mL})$, the mixture was filtered and concentrated under reduced pressure. Purification by flash column chromatography $\left(\mathrm{SiO}_{2}, \mathrm{Et}_{2} \mathrm{O} /\right.$ pentane 1:1) afforded alcohol 7 (4.1 g, $48 \mathrm{mmol}, 96 \%)$ as a colorless liquid.

Reference spectrum: Tetrahedron 2012, 68, 7280-7287.

${ }^{1} \underline{\mathrm{H} \mathrm{NMR}}\left(400 \mathrm{MHz}, \mathrm{CDCl}_{3}\right): \delta 5.49$ (qq, $\left.J=6.7,1.4 \mathrm{~Hz}, 1 \mathrm{H}\right), 3.99(\mathrm{t}, J=1.2 \mathrm{~Hz}, 2 \mathrm{H}), 1.67$ (t, $J=1.3 \mathrm{~Hz}, 3 \mathrm{H}), 1.62(\mathrm{dq}, J=6.7,1.2 \mathrm{~Hz}, 3 \mathrm{H}) \mathrm{ppm}$.

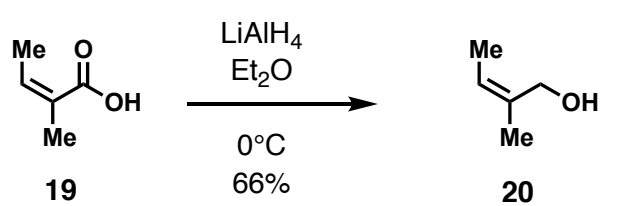

(Z)-2-Methylbut-2-en-1-ol (20). Angelic acid 19 (1.4 g, 14 mmol, 1.0 equiv) in $\mathrm{Et}_{2} \mathrm{O}(5.8 \mathrm{~mL})$ was added dropwise to a suspension of $\mathrm{LiAlH}_{4}\left(1.4 \mathrm{~g}, 38 \mathrm{mmol}, 2.7\right.$ equiv) in $\mathrm{Et}_{2} \mathrm{O}(17 \mathrm{~mL})$ at ambient temperature. After $2 \mathrm{~h}, \mathrm{NaOH}(3 \mathrm{M}, 20 \mathrm{~mL})$ and $\mathrm{Et}_{2} \mathrm{O}(20 \mathrm{~mL})$ were added. Extraction with $\mathrm{Et}_{2} \mathrm{O}(3 \times 40 \mathrm{~mL})$, drying over $\mathrm{Na}_{2} \mathrm{SO}_{4}$, filtration, and concentration under reduced pressure afforded the crude product. Purification by flash column chromatography $\left(\mathrm{SiO}_{2}\right.$, $\mathrm{Et}_{2} \mathrm{O} /$ pentane 1:1) afforded alcohol $20(790 \mathrm{mg}, 9.2 \mathrm{mmol}, 66 \%)$ as a colorless liquid.

Reference spectrum: Org. Lett. 2017, 19, 3703-3706. 
${ }^{1} \underline{\mathrm{H} \mathrm{NMR}}\left(400 \mathrm{MHz}, \mathrm{CDCl}_{3}\right): \delta 5.43-5.32(\mathrm{~m}, 1 \mathrm{H}), 4.15(\mathrm{~d}, J=0.7 \mathrm{~Hz}, 2 \mathrm{H}), 1.78(\mathrm{p}, J=1.5 \mathrm{~Hz}$, $3 \mathrm{H}), 1.68-1.60(\mathrm{~m}, 3 \mathrm{H}) \mathrm{ppm}$.

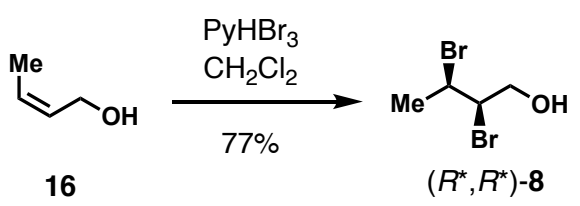

$\left(2 \boldsymbol{R}^{*}, \mathbf{3} \boldsymbol{R}^{*}\right)$-2,3-Dibromobutan-1-ol $(( \pm)-8)$. Pyridinium tribromide (910 mg, $2.8 \mathrm{mmol}$, 1.2 equiv) was added to a solution of olefin 16 (170 mg, $2.4 \mathrm{mmol}, 1.0$ equiv) in $\mathrm{CH}_{2} \mathrm{Cl}_{2}$ $(7 \mathrm{~mL})$, and the mixture was stirred at ambient temperature for $20 \mathrm{~min}$. After addition of $\mathrm{Et}_{2} \mathrm{O}$ $(5 \mathrm{~mL})$ and sat. aq. $\mathrm{Na}_{2} \mathrm{~S}_{2} \mathrm{O}_{3}$ solution $(10 \mathrm{~mL})$, the layers were separated. The organic layer was washed with $\mathrm{HCl}(1 \mathrm{M})$, dried over $\mathrm{Na}_{2} \mathrm{SO}_{4}$, filtered, and concentrated under reduced pressure (30 min at $40{ }^{\circ} \mathrm{C} / 250 \mathrm{mbar}$ ) to afford dibromide $( \pm)-8(420 \mathrm{mg}, 1.8 \mathrm{mmol}, 77 \%$ ) as a brown liquid. Separation of the enantiomers was performed by normal-phase HPLC. The product (200 mg) was dissolved (hexane/EtOH 97:3, $2 \mathrm{~mL}$ ) and then divided into 4 sequential runs on a CHIRALPAK IB column (flow: $18 \mathrm{~mL} / \mathrm{min}$, eluent: hexane/EtOH 97:3). The runs were monitored at a wavelength of $220 \mathrm{~nm}$. The fractions were collected and concentrated under reduced pressure $\left(30 \mathrm{~min}\right.$ at $\left.40{ }^{\circ} \mathrm{C} / 100 \mathrm{mbar}\right)$ to afford $(+)-8\left(64 \mathrm{mg}, t_{\mathrm{R}}=16.07 \mathrm{~min}\right.$, e.r. $=99: 1$, white solid $)$ and $(-)-8\left(70 \mathrm{mg}, t_{\mathrm{R}}=18.65 \mathrm{~min}\right.$, e.r. $=99: 1$, white solid $)$.

TLC: $R_{f}=0.59($ EtOAc/hexane $3: 7) ;{ }^{1}$ H NMR $\left(400 \mathrm{MHz}, \mathrm{CDCl}_{3}\right): \delta 4.49(\mathrm{qd}, J=6.8,3.0 \mathrm{~Hz}$, $1 \mathrm{H}), 4.25$ (ddd, $J=7.1,5.5,3.0 \mathrm{~Hz}, 1 \mathrm{H}), 4.03(\mathrm{dd}, J=12.1,5.5 \mathrm{~Hz}, 1 \mathrm{H}), 3.95(\mathrm{dd}, J=12.1$, $7.1 \mathrm{~Hz}, 1 \mathrm{H}), 1.98-1.89(\mathrm{~m}, 1 \mathrm{H}), 1.81(\mathrm{~d}, J=6.8 \mathrm{~Hz}, 3 \mathrm{H}) \mathrm{ppm} ;{ }^{13} \mathrm{C} \mathrm{NMR}\left(101 \mathrm{MHz}, \mathrm{CDCl}_{3}\right)$ : $\delta$ 65.3, 60.8, 49.0, 23.4 ppm; IR (UATR): v 3369, 2926, 1449, 1380, 1232, 1054, 978, 844, 704, $552 \mathrm{~cm}^{-1}$; Optical Rotation $(+)-8: \quad \alpha_{D}^{23}+41.4 \quad\left(\mathrm{c}=0.50, \quad \mathrm{CHCl}_{3}\right)$; Optical Rotation $(-)-8: \alpha_{D}^{23}-41.7\left(\mathrm{c}=0.50, \mathrm{CHCl}_{3}\right)$; $\underline{\text { HRMS }}(\mathrm{EI})$ : exact mass calculated for $\mathrm{C}_{4} \mathrm{H}_{8} \mathrm{BrO}^{+}\left[\left(M_{-}\right.\right.$ $\left.\mathrm{Br})^{+}\right]$150.9753; found 150.9753 .

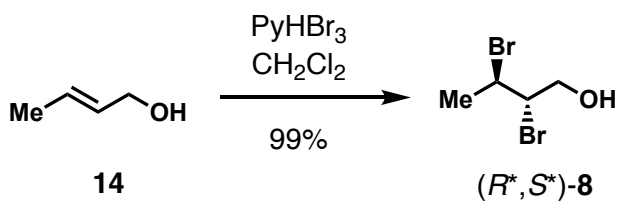

$\left(2 S^{*}, 3 R^{*}\right)$-2,3-Dibromobutan-1-ol $(( \pm)-8)$. Pyridinium tribromide $\quad(2.0 \mathrm{~g}, \quad 6.2 \mathrm{mmol}$, 1.2 equiv) was added to a solution of olefin 3 (370 mg, $5.2 \mathrm{mmol}, 1.0$ equiv) in $\mathrm{CH}_{2} \mathrm{Cl}_{2}$ $(15 \mathrm{~mL})$, and the mixture was stirred at ambient temperature for $20 \mathrm{~min}$. After addition of $\mathrm{Et}_{2} \mathrm{O}$ $(5 \mathrm{~mL})$ and sat. aq. $\mathrm{Na}_{2} \mathrm{~S}_{2} \mathrm{O}_{3}$ solution $(10 \mathrm{~mL})$, the layers were separated. The organic layer was washed with $\mathrm{HCl}(1 \mathrm{M})$, dried over $\mathrm{Na}_{2} \mathrm{SO}_{4}$, filtered, and concentrated under reduced pressure 
(30 min at $40{ }^{\circ} \mathrm{C} / 250 \mathrm{mbar}$ ) to afford dibromide $( \pm)-8(1.2 \mathrm{~g}, 5.1 \mathrm{mmol}, 99 \%$ ) as a brown liquid. Separation of the enantiomers was performed by normal-phase HPLC. The product (200 mg) was dissolved (hexane/EtOH 97:3, $2 \mathrm{~mL}$ ) and then divided into 4 sequential runs on a CHIRALPAK IB column (flow: $18 \mathrm{~mL} / \mathrm{min}$, eluent: hexane/EtOH 97:3). The runs were monitored at a wavelength of $220 \mathrm{~nm}$. The fractions were collected and concentrated under reduced pressure $\left(30 \mathrm{~min}\right.$ at $\left.40{ }^{\circ} \mathrm{C} / 100 \mathrm{mbar}\right)$ to afford $(-)-8\left(49 \mathrm{mg}, t_{\mathrm{R}}=17.85 \mathrm{~min}\right.$, e.r. $=99: 1$, white solid $)$ and $(+)-8\left(37 \mathrm{mg}, t_{\mathrm{R}}=19.48 \mathrm{~min}\right.$, e.r. $=99: 1$, white solid $)$.

TLC: $R_{f}=0.59$ (EtOAc/hexane 3:7); ${ }^{1} \mathrm{H}$ NMR $\left(400 \mathrm{MHz}, \mathrm{CDCl}_{3}\right): \delta 4.38(\mathrm{dq}, J=9.4,6.6 \mathrm{~Hz}$, $1 \mathrm{H}), 4.25$ (ddd, $J=9.4,4.6,3.6 \mathrm{~Hz}, 1 \mathrm{H}), 4.15-4.02(\mathrm{~m}, 2 \mathrm{H}), 1.98$ (t, $J=6.9 \mathrm{~Hz}, 1 \mathrm{H}), 1.91$ (d, $J=6.6 \mathrm{~Hz}, 3 \mathrm{H}) \mathrm{ppm} ;{ }^{13} \mathrm{C} \mathrm{NMR}\left(101 \mathrm{MHz}, \mathrm{CDCl}_{3}\right): \delta 66.3,62.5,47.8,25.7 \mathrm{ppm}$; $\underline{\text { IR (UATR) }}$ $v$ 3370, 2932, 1451,1380,1202, 1155, 1101, 1065, 1009, 936, $545 \mathrm{~cm}^{-1}$; Optical Rotation $(-)-8: \alpha_{D}^{23}-3.0\left(\mathrm{c}=0.50, \mathrm{CHCl}_{3}\right)$; Optical Rotation $(+)-8: \alpha_{D}^{23}+2.7\left(\mathrm{c}=0.50, \mathrm{CHCl}_{3}\right)$; $\underline{\mathrm{HRMS}}$ (EI): exact mass calculated for $\mathrm{C}_{4} \mathrm{H}_{7} \mathrm{Br}_{2}{ }^{+}\left[(M-\mathrm{OH})^{+}\right] 212.8909$; found 212.8909 .

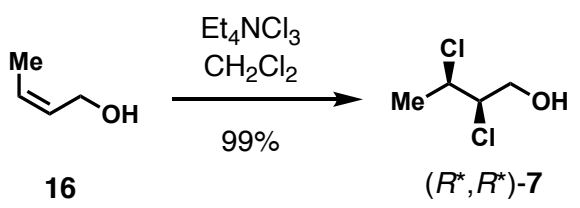

$\left(2 R^{*}, 3 R^{*}\right)-2,3-D i c h l o r o b u t a n-1-o l ~(( \pm)-7) . \quad$ MIOSKOWSKI's reagent $\quad(2.6 \mathrm{~g}, 11 \mathrm{mmol}$, 1.2 equiv) was added to a solution of olefin 16 (400 mg, $5.6 \mathrm{mmol}, 1.0$ equiv) in $\mathrm{CH}_{2} \mathrm{Cl}_{2}$ $(14 \mathrm{~mL})$, and the mixture was stirred at ambient temperature for $20 \mathrm{~min}$. After addition of sat. aq. $\mathrm{NaHCO}_{3}$ solution $(5 \mathrm{~mL})$ and sat. aq. $\mathrm{Na}_{2} \mathrm{~S}_{2} \mathrm{O}_{3}$ solution $(5 \mathrm{~mL})$, the layers were separated and the crude product extracted with pentane $(3 \times 10 \mathrm{~mL})$. The organic layer was dried over $\mathrm{Na}_{2} \mathrm{SO}_{4}$, filtered, and concentrated under reduced pressure $\left(30 \mathrm{~min}\right.$ at $40{ }^{\circ} \mathrm{C} / 250 \mathrm{mbar}$ ) to afford dichloride ( \pm )-13 (780 mg, $5.5 \mathrm{mmol}, 99 \%$ ) as a yellow liquid. Purification was performed by normal-phase HPLC. The product (120 mg) was dissolved (hexane/EtOH 20:1, $1 \mathrm{~mL}$ ) and then divided into 3 sequential runs on a CHIRALPAK IB column (flow: $18 \mathrm{~mL} / \mathrm{min}$, eluent: hexane/EtOH 20:1). The fractions were collected, monitored by thin-layer chromatography $\left(\mathrm{SiO}_{2}, \mathrm{EtOAc} /\right.$ hexane 3:7, $\left.\mathrm{KMnO}_{4}\right)$, and concentrated under reduced pressure $(30 \mathrm{~min}$ at $\left.40{ }^{\circ} \mathrm{C} / 120 \mathrm{mbar}\right)$ to afford dichloride $( \pm)-7(30 \mathrm{mg})$.

TLC: $R_{f}=0.54($ EtOAc/hexane $3: 7) ;{ }^{1} \mathrm{H} \mathrm{NMR}\left(400 \mathrm{MHz}, \mathrm{CDCl}_{3}\right): \delta 4.41(\mathrm{qd}, J=6.7,3.1 \mathrm{~Hz}$, $1 \mathrm{H}), 4.12(\mathrm{ddd}, J=6.9,5.5,3.2 \mathrm{~Hz}, 1 \mathrm{H}), 3.96(\mathrm{dd}, J=11.9,5.5 \mathrm{~Hz}, 1 \mathrm{H}), 3.88(\mathrm{dd}, J=11.8$, $6.9 \mathrm{~Hz}, 1 \mathrm{H}), 1.63(\mathrm{~d}, J=6.7 \mathrm{~Hz}, 3 \mathrm{H}) \mathrm{ppm} ;{ }^{13} \mathrm{C} \mathrm{NMR}\left(101 \mathrm{MHz}, \mathrm{CDCl}_{3}\right): \delta 66.7,64.4,57.0$, 
21.9 ppm; IR (UATR): v 3369, 2934, 1445, 1381, 1262, 1060, 982, 857, 755, 708, 656, 622, $518 \mathrm{~cm}^{-1}$.

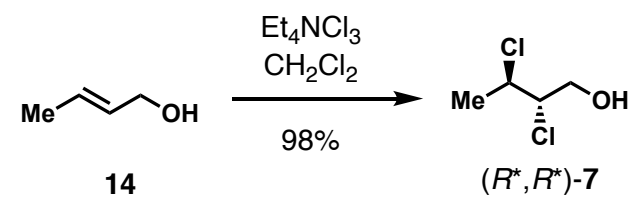

$\left(2 S^{*}, 3 R^{*}\right)$-2,3-Dichlorobutan-1-ol $(( \pm)-7)$. MiOSKOWSKI's reagent $\quad(2.6 \mathrm{~g}, 11 \mathrm{mmol}$, 1.2 equiv) was added to a solution of olefin 14 (390 mg, $5.5 \mathrm{mmol}, 1.0$ equiv) in $\mathrm{CH}_{2} \mathrm{Cl}_{2}$ $(14 \mathrm{~mL})$, and the mixture was stirred at ambient temperature for $20 \mathrm{~min}$. After addition of sat. aq. $\mathrm{NaHCO}_{3}$ solution $(5 \mathrm{~mL})$ and sat. aq. $\mathrm{Na}_{2} \mathrm{~S}_{2} \mathrm{O}_{3}$ solution $(5 \mathrm{~mL})$, the layers were separated and the crude product extracted with pentane $(3 \times 10 \mathrm{~mL})$. The organic layer was dried over $\mathrm{Na}_{2} \mathrm{SO}_{4}$, filtered, and concentrated under reduced pressure $\left(30 \mathrm{~min}\right.$ at $40{ }^{\circ} \mathrm{C} / 250 \mathrm{mbar}$ ) to afford dichloride $( \pm)-7$ (760 mg, $5.3 \mathrm{mmol}, 98 \%)$ as a yellow liquid. Purification was performed by normal-phase HPLC. The product (120 mg) was dissolved (hexane/EtOH 20:1, $1 \mathrm{~mL}$ ) and then divided into 3 sequential runs on a CHIRALPAK IB column (flow: $18 \mathrm{~mL} / \mathrm{min}$, eluent: hexane/EtOH 20:1). The fractions were collected, monitored by thin-layer chromatography $\left(\mathrm{SiO}_{2}, \mathrm{EtOAc} /\right.$ hexane 3:7, $\left.\mathrm{KMnO}_{4}\right)$, and concentrated under reduced pressure $(30 \mathrm{~min}$ at $\left.40{ }^{\circ} \mathrm{C} / 120 \mathrm{mbar}\right)$ to afford dichloride $( \pm)-7(76 \mathrm{mg})$.

TLC: $R_{f}=0.54($ EtOAc/hexane $3: 7) ;{ }^{1} \mathrm{H} \mathrm{NMR}\left(400 \mathrm{MHz}, \mathrm{CDCl}_{3}\right): \delta 4.30-4.18(\mathrm{~m}, 1 \mathrm{H}), 4.07-$ $3.97(\mathrm{~m}, 3 \mathrm{H}), 2.09-1.94(\mathrm{~m}, 1 \mathrm{H}) \mathrm{ppm}, 1.67(\mathrm{~d}, J=6.6 \mathrm{~Hz}, 3 \mathrm{H}) ;{ }^{13} \mathrm{C} \mathrm{NMR}\left(101 \mathrm{MHz}, \mathrm{CDCl}_{3}\right)$ : $\delta$ 68.0, 64.6, 56.3, 22.7 ppm; IR (UATR): v 3369, 2938, 1451, 1382, 1196, 1106, 1071, 1017 , $948,854,751,656 \mathrm{~cm}^{-1}$.

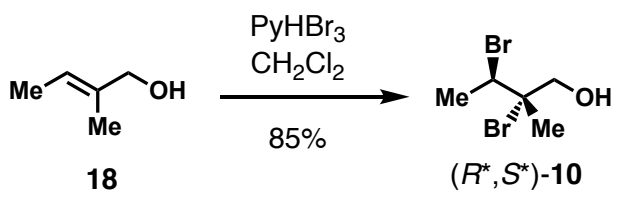

$\left(2 S^{*}, 3 R^{*}\right)$-2,3-Dibromo-2-methylbutan-1-ol $\quad(( \pm)-10)$. Pyridinium tribromide $(2.2 \mathrm{~g}$, $7.0 \mathrm{mmol}, 1.2$ equiv) was added to a solution of olefin 18 (500 mg, $5.8 \mathrm{mmol}, 1.0$ equiv) in $\mathrm{CH}_{2} \mathrm{Cl}_{2}(17 \mathrm{~mL})$, and the mixture was stirred at ambient temperature for $20 \mathrm{~min}$. After addition of $\mathrm{Et}_{2} \mathrm{O}(5 \mathrm{~mL})$ and sat. aq. $\mathrm{Na}_{2} \mathrm{~S}_{2} \mathrm{O}_{3}$ solution $(10 \mathrm{~mL})$, the layers were separated. The organic layer was washed with $\mathrm{HCl}(1 \mathrm{M})$, dried over $\mathrm{Na}_{2} \mathrm{SO}_{4}$, filtered, and concentrated under reduced pressure (30 $\mathrm{min}$ at $40{ }^{\circ} \mathrm{C} / 250 \mathrm{mbar}$ ) to afford the crude product. Purification by flash column chromatography $\left(\mathrm{SiO}_{2}, \mathrm{Et}_{2} \mathrm{O} /\right.$ pentane $\left.1: 1\right)$ was followed by dilution of the product with $\mathrm{MeCN}$ 
$(2 \mathrm{~mL})$, and filtration through a syringe filter and concentration under reduced pressure (20 min at $\left.40{ }^{\circ} \mathrm{C} / 120 \mathrm{mbar}\right)$ afforded $( \pm)-\mathbf{1 0}(1.2 \mathrm{~g}, 4.9 \mathrm{mmol}, 85 \%)$ as a grey solid. Further purification was performed by normal-phase HPLC. The product $(100 \mathrm{mg})$ was dissolved (hexane/EtOH 97:3, $1 \mathrm{~mL}$ ) and then divided into 2 sequential runs on a CHIRALPAK IB column (flow: $18 \mathrm{~mL} / \mathrm{min}$, eluent: hexane/EtOH 97:3). The runs were monitored at a wavelength of $220 \mathrm{~nm}$. The fractions were collected and concentrated under reduced pressure $(20 \mathrm{~min}$ at $\left.40{ }^{\circ} \mathrm{C} / 120 \mathrm{mbar}\right)$ to afford $( \pm)-\mathbf{1 0}\left(75 \mathrm{mg}, t_{\mathrm{R}}=10.7 \mathrm{~min}\right)$.

${ }^{1} \mathrm{H} \mathrm{NMR}\left(400 \mathrm{MHz}, \mathrm{CDCl}_{3}\right): \delta 4.68(\mathrm{q}, J=6.8 \mathrm{~Hz}, 1 \mathrm{H}), 3.94(\mathrm{~d}, J=12.7 \mathrm{~Hz}, 1 \mathrm{H}), 3.82(\mathrm{~d}$, $J=12.7 \mathrm{~Hz}, 1 \mathrm{H}), 1.92(\mathrm{~d}, J=6.8 \mathrm{~Hz}, 3 \mathrm{H}), 1.77(\mathrm{~s}, 3 \mathrm{H}) \mathrm{ppm} ;{ }^{13} \mathrm{C} \mathrm{NMR}\left(101 \mathrm{MHz}, \mathrm{CDCl}_{3}\right)$ : $\delta$ 76.0, 72.0, 53.1, 22.9, 21.8 ppm; IR (UATR): v 3378, 2979, 2934, 2872, 1451, 1379, 1214, 1160, 1063, 1025, 980, 963, 908, 803, 688, 615, 574, $537 \mathrm{~cm}^{-1}$; $\underline{\text { HRMS }}$ (EI): exact mass calculated for $\mathrm{C}_{5} \mathrm{H}_{10} \mathrm{BrO}^{+}\left[(M-\mathrm{Br})^{+}\right]$164.9910; found 164.9910.

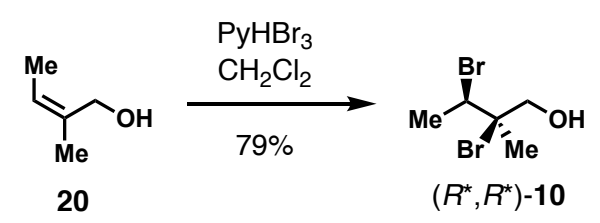

$\left(2 R^{*}, 3 R^{*}\right)-2,3-D i b r o m o-2-m e t h y l b u t a n-1-o l ~(( \pm)-10)$. Pyridinium tribromide $(890 \mathrm{mg}$, $2.8 \mathrm{mmol}, 1.2$ equiv) was added to a solution of olefin 20 (200 mg, $2.3 \mathrm{mmol}, 1.0$ equiv) in $\mathrm{CH}_{2} \mathrm{Cl}_{2}(6.6 \mathrm{~mL})$, and the mixture was stirred at ambient temperature for $20 \mathrm{~min}$. After addition of $\mathrm{Et}_{2} \mathrm{O}(5 \mathrm{~mL})$ and sat. aq. $\mathrm{Na}_{2} \mathrm{~S}_{2} \mathrm{O}_{3}$ solution $(10 \mathrm{~mL})$, the layers were separated. The organic layer was washed with $\mathrm{HCl}(1 \mathrm{M})$, dried over $\mathrm{Na}_{2} \mathrm{SO}_{4}$, filtered, and concentrated under reduced pressure (30 min at $40{ }^{\circ} \mathrm{C} / 250$ mbar) to afford the crude product. Purification by flash column chromatography $\left(\mathrm{SiO}_{2}, \mathrm{Et}_{2} \mathrm{O} /\right.$ pentane 1:4, then 1:1) afforded $( \pm)-\mathbf{1 0}(450 \mathrm{mg}, 1.8 \mathrm{mmol}, 79 \%)$ as a white solid. Further purification and separation of the enantiomers was performed by normal-phase HPLC. The product (200 mg) was dissolved (hexane/EtOH 97:3, $2 \mathrm{~mL}$ ) and then divided into 4 sequential runs on a CHIRALPAK IB column (flow: $18 \mathrm{~mL} / \mathrm{min}$, eluent: hexane/EtOH 97:3). The runs were monitored at a wavelength of $220 \mathrm{~nm}$. Two runs were performed to collect the racemate $(80 \mathrm{mg})$ and two runs were used to separate the enantiomers: $(+)-10\left(38 \mathrm{mg}, t_{\mathrm{R}}=15.94 \mathrm{~min}\right.$, e.r. $=99: 1$, white solid) and (-)-10 (34 mg, $t_{\mathrm{R}}=17.79 \mathrm{~min}$, e.r. $=99: 1$, white solid).

${ }^{1}$ H NMR $\left(400 \mathrm{MHz}, \mathrm{CDCl}_{3}\right): \delta 4.44(\mathrm{q}, J=6.8 \mathrm{~Hz}, 1 \mathrm{H}), 3.95-3.85(\mathrm{~m}, 2 \mathrm{H}), 1.99(\mathrm{td}, J=7.1$, $0.8 \mathrm{~Hz}, 1 \mathrm{H}), 1.91-1.83(\mathrm{~m}, 6 \mathrm{H}) \mathrm{ppm} ;{ }^{13} \mathrm{C} \mathrm{NMR}\left(101 \mathrm{MHz}, \mathrm{CDCl}_{3}\right): \delta 74.5,70.1,56.1,27.1$, 23.0 ppm; IR (UATR): v 3391, 2979, 2935, 2872, 1442, 1380, 1308, 1205, 1145, 1090, 1068, 
1024, 976, 959, 908, 806, 733, 668, 637, 593, 562, 536, $514 \mathrm{~cm}^{-1}$; Optical Rotation (+)-10: $\alpha_{D}^{23}$ $+4.8\left(\mathrm{c}=0.50, \mathrm{CHCl}_{3}\right)$; Optical Rotation $(-)-10: \alpha_{D}^{23}-4.9\left(\mathrm{c}=0.50, \mathrm{CHCl}_{3}\right)$; $\underline{\text { HRMS }}(\mathrm{EI})$ : exact mass calculated for $\mathrm{C}_{5} \mathrm{H}_{10} \mathrm{BrO}^{+}\left[(\mathrm{M}-\mathrm{Br})^{+}\right]$164.9910; found 164.9910.

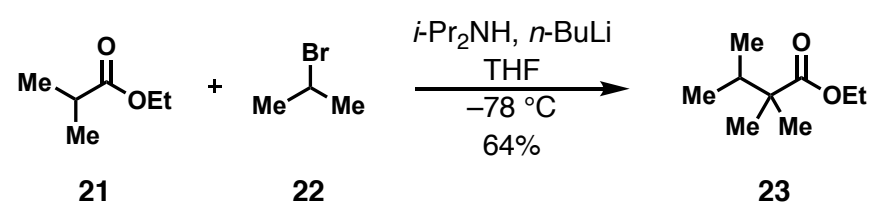

Ethyl 2,2,3-trimethylbutanoate (23). $n$-BuLi (1.6 M in hexane, $30 \mathrm{~mL}, 48 \mathrm{mmol}, 1.3$ equiv) was added at $0{ }^{\circ} \mathrm{C}$ to a solution of diisopropylamine $(8.0 \mathrm{~mL}, 56 \mathrm{mmol}, 1.5$ equiv) in $\mathrm{THF}$ $(45 \mathrm{~mL})$, and the mixture was stirred at that temperature for $30 \mathrm{~min}$. Ethyl isobutyrate 21 (5.0 mL, $37 \mathrm{mmol}, 1.0$ equiv) was added dropwise at $-78^{\circ} \mathrm{C}$. The mixture was stirred at that temperature for $1 \mathrm{~h}$. Subsequently, 2-bromopropane $22(11 \mathrm{~mL}, 110 \mathrm{mmol}, 3.0$ equiv) was added dropwise to the mixture. The reaction mixture was allowed to reach ambient temperature over $48 \mathrm{~h}$. After dilution with $\mathrm{Et}_{2} \mathrm{O}(50 \mathrm{~mL})$ and addition of sat. aq. $\mathrm{NH}_{4} \mathrm{Cl}$ solution $(30 \mathrm{~mL})$, the layers were separated. The crude product was extracted with $\mathrm{Et}_{2} \mathrm{O}(3 \times 50 \mathrm{~mL})$, dried over $\mathrm{Na}_{2} \mathrm{SO}_{4}$, filtered, and concentrated under reduced pressure. Distillation $\left(155^{\circ} \mathrm{C}, 1 \mathrm{~atm}\right)$ afforded product 23 (3.7 g, $24 \mathrm{mmol}, 64 \%$ ) as a colorless liquid.

${ }^{1} \underline{\mathrm{H} \mathrm{NMR}}\left(400 \mathrm{MHz}, \mathrm{CDCl}_{3}\right): \delta 4.12(\mathrm{q}, J=7.1 \mathrm{~Hz}, 2 \mathrm{H}), 1.97$ (hept, $\left.J=6.9 \mathrm{~Hz}, 1 \mathrm{H}\right), 1.24$ (t, $J=7.1 \mathrm{~Hz}, 3 \mathrm{H}), 1.08(\mathrm{~s}, 6 \mathrm{H}), 0.84(\mathrm{~d}, J=6.9 \mathrm{~Hz}, 6 \mathrm{H}) \mathrm{ppm} ;{ }^{13} \mathrm{C} \mathrm{NMR}\left(101 \mathrm{MHz}, \mathrm{CDCl}_{3}\right)$ : $\delta 178.4,60.2,45.7,35.0,21.6$ (2C), 17.7 (2C), 14.4 ppm; IR (UATR): v 2967, 2879, 1727, 1465, 1383, 1371, 1334, 1264, 1221, 1158, 1131, 1097, 1065, 1027, 909, 857, 797, 775, 616, $517 \mathrm{~cm}^{-1}$; $\underline{\text { HRMS }}$ (EI): exact mass calculated for $\mathrm{C}_{6} \mathrm{H}_{12} \mathrm{O}_{2}{ }^{+}\left[\left(M-\mathrm{C}_{3} \mathrm{H}_{6}\right)^{+}\right] 116.0837$; found 116.0832 .

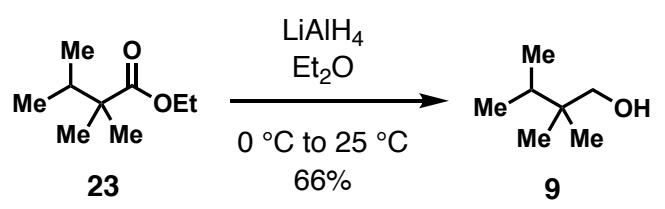

2,2,3-Trimethylbutan-1-ol (9). Ethyl enoate 23 (3.4 g, 21 mmol, 1.0 equiv) in $\mathrm{Et}_{2} \mathrm{O}(4 \mathrm{~mL})$ was added at $0{ }^{\circ} \mathrm{C}$ to a suspension of $\mathrm{LiAlH}_{4}\left(1.8 \mathrm{~g}, 47 \mathrm{mmol}, 2.2\right.$ equiv) in $\mathrm{Et}_{2} \mathrm{O}$ (42 mL), and the mixture was allowed to reach ambient temperature over $18 \mathrm{~h}$. After addition of $\mathrm{NaOH}(3 \mathrm{M}$, $10 \mathrm{~mL})$ and Rochelle'salt solution $(20 \mathrm{~mL})$, the mixture was further diluted with $\mathrm{Et}_{2} \mathrm{O}(20 \mathrm{~mL})$. After stirring for $30 \mathrm{~min}$, the layers were separated. The organic layer was dried over $\mathrm{Na}_{2} \mathrm{SO}_{4}$, 
filtered, and concentrated under reduced pressure. Distillation $\left(160^{\circ} \mathrm{C}, 1 \mathrm{~atm}\right)$ afforded product $9(1.6 \mathrm{~g}, 14 \mathrm{mmol}, 66 \%)$ as a colorless liquid.

${ }^{1} \mathrm{H} \mathrm{NMR}\left(400 \mathrm{MHz}, \mathrm{CDCl}_{3}\right): \delta 3.38$ (s, 2H), 1.63 (hept, $\left.J=6.9 \mathrm{~Hz}, 1 \mathrm{H}\right), 1.34$ (s, 1H), 0.86 (d, $J=6.9 \mathrm{~Hz}, 6 \mathrm{H}), 0.82(\mathrm{~s}, 6 \mathrm{H}) \mathrm{ppm} ;{ }^{13} \mathrm{C} \mathrm{NMR}\left(101 \mathrm{MHz}, \mathrm{CDCl}_{3}\right): \delta 71.2,37.5,32.7,21.1(2 \mathrm{C})$, 17.5 (2C) ppm; IR (UATR): v 3350, 2960, 2876, 1467, 1392, 1379, 1367, 1272, 1206, 1176, $1151,1102,1029,967,903,844,826,571 \mathrm{~cm}^{-1}$; $\underline{\mathrm{HRMS}}$ (EI): exact mass calculated for $\mathrm{C}_{6} \mathrm{H}_{13}{ }^{+}$ $\left[\left(M-\mathrm{CH}_{3} \mathrm{O}\right)^{+}\right]$85.1012; found 85.1012.

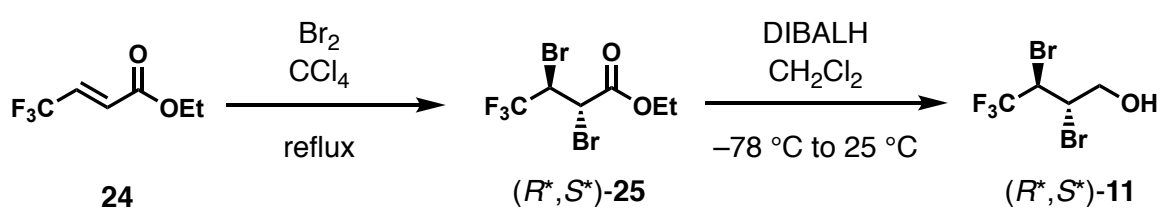

(2S*,3R*)-2,3-Dibromo-4,4,4-trifluorobutan-1-ol $\quad(( \pm)-11) . \quad \mathrm{Br}_{2} \quad(0.57 \mathrm{~mL}, 11 \mathrm{mmol}$, 1.1 equiv) was added to a solution of olefin $24\left(1.69 \mathrm{~g}, 10.0 \mathrm{mmol}, 1.0\right.$ equiv) in $\mathrm{CCl}_{4}(30 \mathrm{~mL})$, and the mixture was heated to reflux for $5 \mathrm{~h}$. The solvent was removed under reduced pressure. The crude dibromide $( \pm)-25$ was then dissolved in $\mathrm{CH}_{2} \mathrm{Cl}_{2}(20 \mathrm{~mL})$ and cooled to $-78^{\circ} \mathrm{C}$. DIBALH ( $1 \mathrm{M}$ in $\mathrm{CH}_{2} \mathrm{Cl}_{2}, 12 \mathrm{~mL}, 1.2 \mathrm{mmol}, 1.2$ equiv) was added and the reaction mixture was stirred at $0{ }^{\circ} \mathrm{C}$ for $40 \mathrm{~min}$. The mixture was quenched with sat. aq. $\mathrm{Na}_{2} \mathrm{~S}_{2} \mathrm{O}_{3}$ solution and stirred for $2 \mathrm{~h}$ resulting in the crude product of $( \pm)-11(2.62 \mathrm{~g})$. Separation was performed by normal-phase HPLC. The product (500 mg) was dissolved (hexane/EtOH 97:3, $5 \mathrm{~mL}$ ) and then divided into several sequential runs on a CHIRALPAK IB column (flow: $18 \mathrm{~mL} / \mathrm{min}$, eluent: hexane/EtOH 97:3). The runs were monitored at a wavelength of $220 \mathrm{~nm}$. The fractions were collected and concentrated under reduced pressure $\left(1 \mathrm{~h}\right.$ at $\left.30^{\circ} \mathrm{C} / 80-90 \mathrm{mbar}\right)$ to afford $233 \mathrm{mg}$. TLC: $R_{f}=0.45$ (EtOAc/hexane 3:7); ${ }^{1} \underline{\mathrm{H} \text { NMR }}\left(400 \mathrm{MHz}, \mathrm{CDCl}_{3}\right): \delta 4.67-4.60(\mathrm{~m}, 1 \mathrm{H}), 4.39$ $(\mathrm{td}, J=6.1,3.7 \mathrm{~Hz}, 1 \mathrm{H}), 4.14-4.03(\mathrm{~m}, 2 \mathrm{H}) \mathrm{ppm} ;{ }^{13} \underline{\mathrm{C} \mathrm{NMR}}\left(101 \mathrm{MHz}, \mathrm{CDCl}_{3}\right): \delta 65.6,50.3$, 48.7 (q, $J=32.1)$ ppm; ${ }^{19} \underline{\mathrm{F} \mathrm{NMR}}\left(377 \mathrm{MHz}, \mathrm{CDCl}_{3}\right):-66.8 \mathrm{ppm}$.

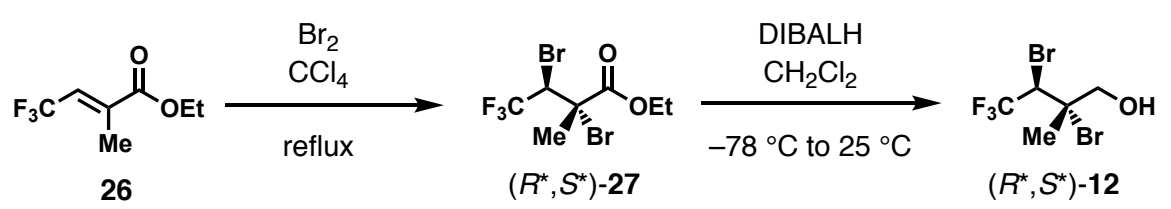

$\left(\left(2 R^{*}, 3 R^{*}\right)-2,3-D i b r o m o-4,4,4-t r i f l u o r o-2-m e t h y l b u t a n-1-o l ~(( \pm)-12) . \quad \mathrm{Br}_{2} \quad(0.62 \mathrm{~mL}\right.$, $12 \mathrm{mmol}, 2.2$ equiv) was added to a solution of olefin 26 (1.0 g, $5.49 \mathrm{mmol}, 1.0$ equiv) in $\mathrm{CCl}_{4}$ $(17 \mathrm{~mL})$, and the mixture was heated to reflux for $5 \mathrm{~h}$. The solvent was removed under reduced pressure. The crude dibromide $( \pm)-27$ was then dissolved in $\mathrm{CH}_{2} \mathrm{Cl}_{2}(11 \mathrm{~mL})$ and cooled to $0{ }^{\circ} \mathrm{C}$. DIBALH ( $1 \mathrm{M}$ in $\mathrm{CH}_{2} \mathrm{Cl}_{2}, 8.2 \mathrm{~mL}, 8.2 \mathrm{mmol}, 1.5$ equiv) was added and the reaction 
mixture was stirred at $0{ }^{\circ} \mathrm{C}$ for $30 \mathrm{~min}$. The mixture was quenched with sat. aq. $\mathrm{Na}_{2} \mathrm{~S}_{2} \mathrm{O}_{3}$ solution and stirred for $2 \mathrm{~h}$ resulting in the crude product of $( \pm)-12(1.6 \mathrm{~g})$. Purification was performed by normal-phase HPLC. The product (500 mg) was dissolved (hexane/EtOH 96:4, $5 \mathrm{~mL}$ ) and then divided into several sequential runs on a CHIRALPAK IB column (flow: $18 \mathrm{~mL} / \mathrm{min}$, eluent: hexane/EtOH 96:4). The runs were monitored at a wavelength of $220 \mathrm{~nm}$. The combined fractions were collected and concentrated under reduced pressure to afford $149 \mathrm{mg}$.

TLC: $R_{f}=0.48$ (EtOAc/hexane 3:7); ${ }^{1} \underline{\mathrm{H} N M R}\left(400 \mathrm{MHz}, \mathrm{CDCl}_{3}\right): \delta 4.85$ (q, $\left.J=7.1,1 \mathrm{H}\right), 3.91$ (dd, $J=100.5,12.6 \mathrm{~Hz}, 1 \mathrm{H}), 1.85$ (br. s, $3 \mathrm{H}) \mathrm{ppm} ;{ }^{13} \mathrm{C} \mathrm{NMR}\left(101 \mathrm{MHz}, \mathrm{CDCl}_{3}\right): \delta 72.4,65.1$, 52.1 (q, $J=30.4 \mathrm{~Hz}), 23.4 \mathrm{ppm} ;{ }^{19} \mathrm{~F} \mathrm{NMR}\left(377 \mathrm{MHz}, \mathrm{CDCl}_{3}\right):-62.2 \mathrm{ppm}$.

\section{S3. Methods for Solution Binding Studies}

Preparation of solutions for ECD titrations: A solution of the AAC $(P)_{4}$ or $(M)_{4}-\mathbf{1}$, referred to as "host", was prepared gravimetrically $(\sim 10 \mu \mathrm{M})$ in $n$-octane. The parent solution was used to prepare the stock solution of the varied component, referred to as "guest" (2-12). All studies were carried out under air atmosphere without drying of the solvents prior to use. Portions of the guest solution were added stepwise, and the ECD spectrum was recorded after each addition. During titration, the change in $\Delta \Delta \varepsilon\left(\mathrm{M}^{-1} \mathrm{~cm}^{-1}\right)$ was followed at the maximum of the highest wavelength (302-306 nm).

Determination of the association constant: During ECD titrations, the change in $\Delta \varepsilon$ $\left(\mathrm{M}^{-1} \mathrm{~cm}^{-1}\right)$ was followed at the maximum intensity between $302-306 \mathrm{~nm}$. The change in $\Delta \varepsilon$ $\left(\mathrm{M}^{-1} \mathrm{~cm}^{-1}\right)$ was plotted against the guest concentration and curve-fitted to a $1: 1$ binding isotherm.

The data was curve-fitted using the software IGOR Pro V6.12 according to the following equation. ${ }^{[6]}$

$$
\Delta \delta=\frac{\Delta \delta_{\text {sat }}}{2}\left[\left(\frac{[G]_{0}}{[H]_{0}}+1+\frac{1}{K_{a}[H]_{0}}\right)-\sqrt{\left.\left(\frac{[G]_{0}}{[H]_{0}}+1+\frac{1}{K_{a}[H]_{0}}\right)^{2}+\frac{[G]_{0}}{[H]_{0}}\right]}\right.
$$

with $\Delta \delta=\left(\delta_{s a t}-\delta_{0}\right) \frac{[C]}{[H]_{0}}$

where the following parameters were applied:

$\Delta \delta \quad$ Change in relative $\operatorname{shift}(\Delta \Delta \varepsilon)$ to the free host

$\Delta \delta_{\text {sat }} \quad$ Calculated change in chemical shift at saturation binding $\left(\Delta \Delta \varepsilon_{\text {sat }}\right)$ 


$\begin{array}{ll}{[H]_{0}} & \text { Constant host concentration }(\mathrm{M}) \\ {[G]} & \text { Guest concentration }(\mathrm{M}) \\ K_{\mathrm{a}} & \text { Association constant }\left(\mathrm{M}^{-1}\right)\end{array}$

The calculated fitting error for all measurements is given below each binding isotherm. All fitting errors are well within $\pm 15 \%\left(K_{\mathrm{a}}\right)$. Due to the preparation process of the solutions the actual error of the measurement was estimated to be higher than the actual fitting errors. The overall error in $K_{\mathrm{a}}$ is therefore estimated to be $\pm 20 \%$. The authors are well aware that the error value is likely to be overestimated.

Preparation of solutions for ITC titrations: A solution of the AAC $(P)_{4}-\mathbf{1}$ or $(M)_{4}-\mathbf{1}$, referred to as "host", was prepared gravimetrically $(\sim 0.1 \mathrm{mM})$ in $n$-octane. The "guest" solution was prepared in concentrations between 1-10 $\mathrm{mM}$ depending on the order of magnitude of the expected binding constant. All studies were carried out under air atmosphere without drying of the solvents prior to use at $303 \mathrm{~K}$.

From ITC experiments, the thermodynamic quantities of $K_{\mathrm{a}}, \Delta G, \Delta H$, and $\Delta S$ were obtained.

\section{S4. Titration Data and Binding Isotherms Obtained by ECD Spectroscopic Titrations}

\section{Butan-1-ol 2}

Table S1. Experimental values for the determination of the association constant $K_{\mathrm{a}}$ of AAC $(P)_{4}-\mathbf{1}$ with butan-1ol 2 in $n$-octane at $293 \mathrm{~K}$.

\begin{tabular}{ccccccc}
\hline & $\begin{array}{c}K_{\mathrm{a}} \\
\mathrm{M}^{-1}\end{array}$ & $\begin{array}{c}\mathrm{AAC}(P)_{4}-\mathbf{1} \\
(\mu \mathrm{M})\end{array}$ & $\begin{array}{c}\text { [guest }]_{\max } \\
(\mathrm{mM})\end{array}$ & $\begin{array}{c}\text { guest } \\
\text { equiv.max }\end{array}$ & $\begin{array}{c}\Delta \Delta \varepsilon_{\max } \\
\left(\mathrm{M}^{-1} \mathrm{~cm}^{-1}\right)\end{array}$ & $\begin{array}{c}\Delta \Delta \varepsilon_{\text {sat }} \\
\left(\mathrm{M}^{-1} \mathrm{~cm}^{-1}\right)\end{array}$ \\
\hline $\mathrm{ECD}$ & - & 8.8 & 5.4 & 611.6 & - & - \\
\hline
\end{tabular}

\section{3-Methylbutan-1-ol 3}

Table S2. Experimental values for the determination of the association constant $K_{\mathrm{a}}$ of AAC $(P)_{4}-1$ with 3methylbutan-1-ol 3 in $n$-octane at $293 \mathrm{~K}$.

\begin{tabular}{ccccccc}
\hline $\begin{array}{c}K_{\mathrm{a}} \\
\mathrm{M}^{-1}\end{array}$ & $\begin{array}{c}\mathrm{AAC}(P)_{4}-\mathbf{1} \\
(\mu \mathrm{M})\end{array}$ & $\begin{array}{c}\text { [guest }]_{\max } \\
(\mathrm{mM})\end{array}$ & $\begin{array}{c}\text { guest } \\
\text { equiv.max }\end{array}$ & $\begin{array}{c}\Delta \Delta \varepsilon_{\max } \\
\left(\mathrm{M}^{-1} \mathrm{~cm}^{-1}\right)\end{array}$ & $\begin{array}{c}\Delta \Delta \varepsilon_{\text {sat }} \\
\left(\mathrm{M}^{-1} \mathrm{~cm}^{-1}\right)\end{array}$ \\
\hline ECD & $1.5 \cdot 10^{3}$ & 8.8 & 3.3 & 376.3 & 83.8 & 102.9 \\
\hline
\end{tabular}




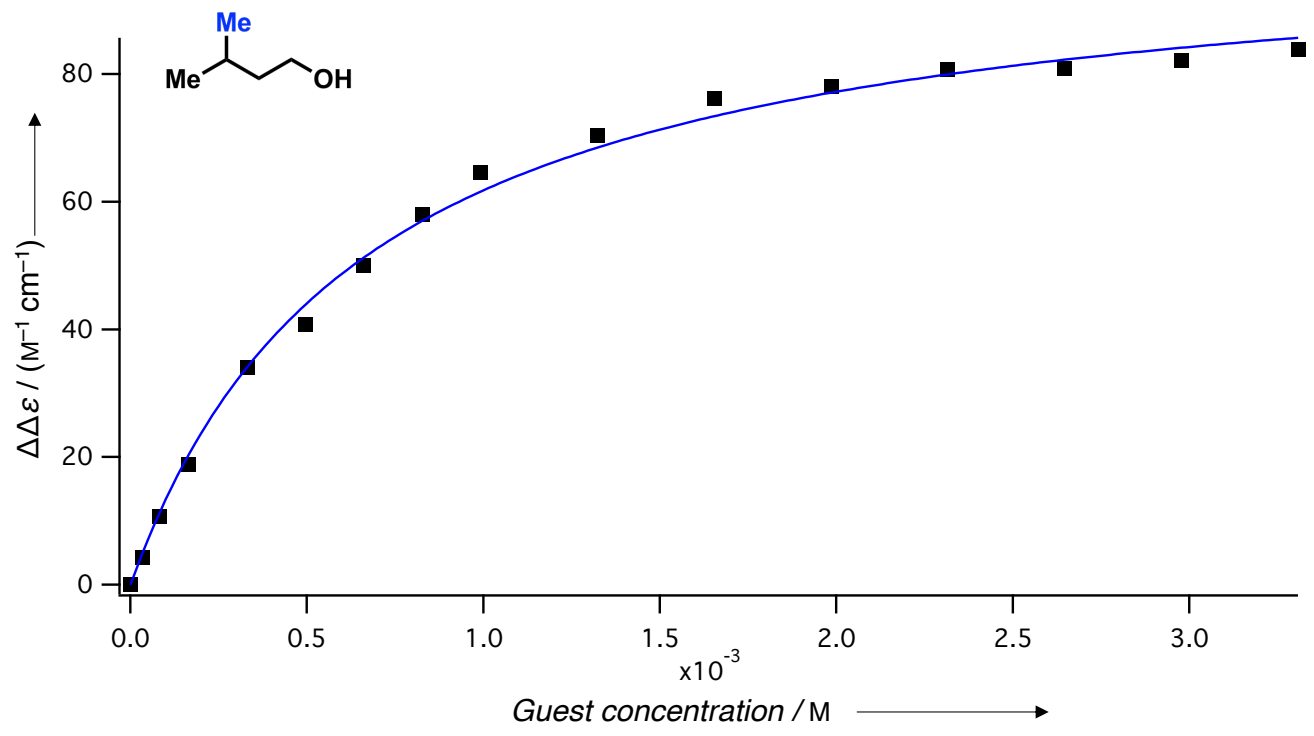

Figure S2. ECD binding isotherm of AAC $(P)_{4}-\mathbf{1}$ with 3-methylbutan-1-ol 3 in $n$-octane at $293 \mathrm{~K} . K_{\mathrm{a}}=1.51 \cdot 10^{3}$ $\pm 0.09 \cdot 10^{3}, \Delta \Delta \varepsilon_{\max }=83.8 \mathrm{M}^{-1} \mathrm{~cm}^{-1}, \Delta \Delta \varepsilon_{\mathrm{sat}}=102.9 \pm 1.9 \mathrm{M}^{-1} \mathrm{~cm}^{-1}$.

\section{( \pm )-2-Methylbutan-1-ol $(R / S)-4$}

Table S3. Experimental values for the determination of the association constant $K_{\mathrm{a}}$ of AAC $(P)_{4}-1$ with $( \pm)$-2methylbutan-1-ol $(R / S)-4$ in $n$-octane at $293 \mathrm{~K}$.

\begin{tabular}{ccccccc}
\hline $\begin{array}{c}K_{\mathrm{a}} \\
\mathrm{M}^{-1}\end{array}$ & $\begin{array}{c}\mathrm{AAC}(P)_{4}-\mathbf{1} \\
(\mu \mathrm{M})\end{array}$ & $\begin{array}{c}\text { [guest }]_{\max } \\
(\mathrm{mM})\end{array}$ & $\begin{array}{c}\text { guest } \\
\text { equiv.max }\end{array}$ & $\begin{array}{c}\Delta \Delta \varepsilon_{\max } \\
\left(\mathrm{M}^{-1} \mathrm{~cm}^{-1}\right)\end{array}$ & $\begin{array}{c}\Delta \Delta \varepsilon_{\text {sat }} \\
\left(\mathrm{M}^{-1} \mathrm{~cm}^{-1}\right)\end{array}$ \\
\hline ECD & $2.3 \cdot 10^{3}$ & 9.6 & 3.1 & 326.7 & 64.1 & 76.4 \\
\hline
\end{tabular}

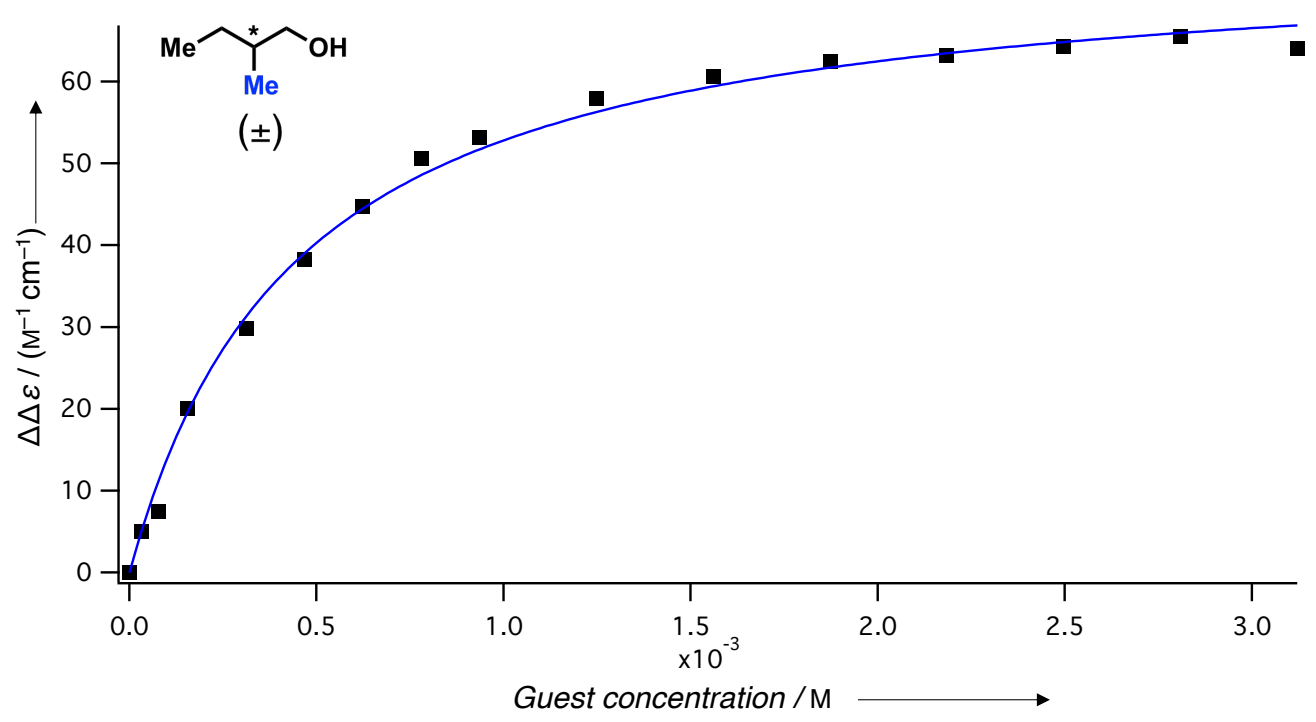

Figure S3. ECD binding isotherm of AAC $(P)_{4}-1$ with $( \pm)$-2-methylbutan-1-ol $(R / S)-4$ in $n$-octane at $293 \mathrm{~K} . K_{\mathrm{a}}$ $=2.26 \cdot 10^{3} \pm 0.14 \cdot 10^{3}, \Delta \Delta \varepsilon_{\max }=64.1 \mathrm{M}^{-1} \mathrm{~cm}^{-1}, \Delta \Delta \varepsilon_{\mathrm{sat}}=76.4 \pm 1.3 \mathrm{M}^{-1} \mathrm{~cm}^{-1}$. 


\section{2,2-Dimethylbutan-1-ol 5}

Table S4. Experimental values for the determination of the association constant $K_{\mathrm{a}}$ of AAC $(P)_{4}-1$ with 2,2dimethylbutan-1-ol 5 in $n$-octane at $293 \mathrm{~K}$.

\begin{tabular}{ccccccc}
\hline $\begin{array}{c}K_{\mathrm{a}} \\
\mathrm{M}^{-1}\end{array}$ & $\begin{array}{c}\mathrm{AAC}(P)_{4}-\mathbf{1} \\
(\mu \mathrm{M})\end{array}$ & $\begin{array}{c}\text { [guest }]_{\max } \\
(\mathrm{mM})\end{array}$ & $\begin{array}{c}\text { guest } \\
\text { equiv.max }\end{array}$ & $\begin{array}{c}\Delta \Delta \varepsilon_{\max } \\
\left(\mathrm{M}^{-1} \mathrm{~cm}^{-1}\right)\end{array}$ & $\begin{array}{c}\Delta \Delta \varepsilon_{\text {sat }} \\
\left(\mathrm{M}^{-1} \mathrm{~cm}^{-1}\right)\end{array}$ \\
\hline $\mathrm{ECD}$ & $2.0 \cdot 10^{4}$ & 8.9 & 2.6 & 293.1 & 102.0 & 106.1 \\
\hline
\end{tabular}

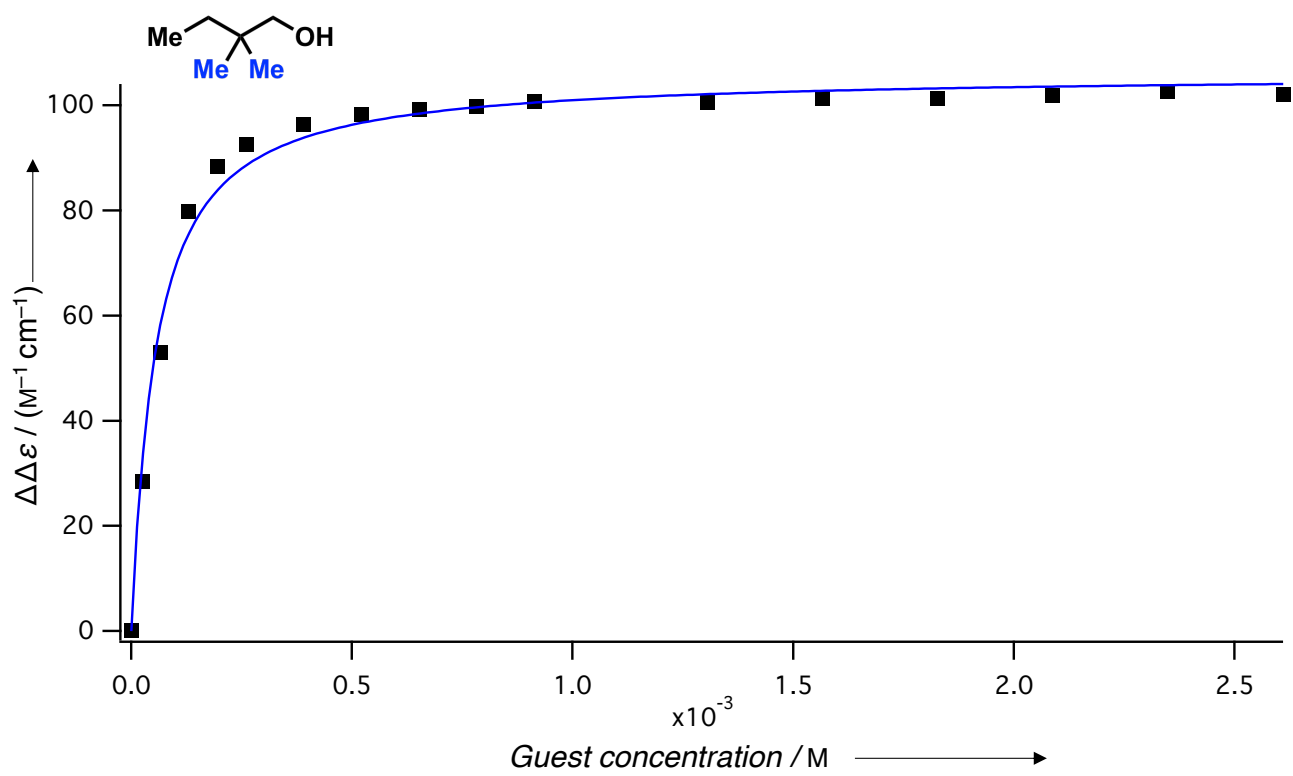

Figure S4. ECD binding isotherm of AAC $(P)_{4}-1$ with 2,2-dimethylbutan-1-ol 5 in $n$-octane at $293 \mathrm{~K} . K_{\mathrm{a}}=$ $2.01 \cdot 10^{4} \pm 0.16 \cdot 10^{4}, \Delta \Delta \varepsilon_{\max }=102.0 \mathrm{M}^{-1} \mathrm{~cm}^{-1}, \Delta \Delta \varepsilon_{\mathrm{sat}}=106.1 \pm 1.1 \mathrm{M}^{-1} \mathrm{~cm}^{-1}$.

\section{( \pm )-2,3-Methylbutan-1-ol $(R / S)$-6}

Table S5. Experimental values for the determination of the association constant $K_{\mathrm{a}}$ of AAC $(P)_{4}-\mathbf{1}$ with $( \pm)-2,3-$ dimethylbutan-1-ol $(R / S)$-6 in $n$-octane at $293 \mathrm{~K}$.

\begin{tabular}{ccccccc}
\hline$K_{\mathrm{a}}$ & $\mathrm{AAC}(P)_{4}-\mathbf{1}$ \\
$\mathrm{M}^{-1}$ & $(\mu \mathrm{M})$ & $\begin{array}{c}\text { [guest }]_{\max } \\
(\mathrm{mM})\end{array}$ & $\begin{array}{c}\text { guest } \\
\text { equiv.max }\end{array}$ & $\begin{array}{c}\Delta \Delta \varepsilon_{\max } \\
\left(\mathrm{M}^{-1} \mathrm{~cm}^{-1}\right)\end{array}$ & $\begin{array}{c}\Delta \Delta \varepsilon_{\text {sat }} \\
\left(\mathrm{M}^{-1} \mathrm{~cm}^{-1}\right)\end{array}$ \\
\hline ECD & $9.9 \cdot 10^{3}$ & 9.1 & 1.4 & 156.1 & 59.4 & 65.5 \\
\hline
\end{tabular}




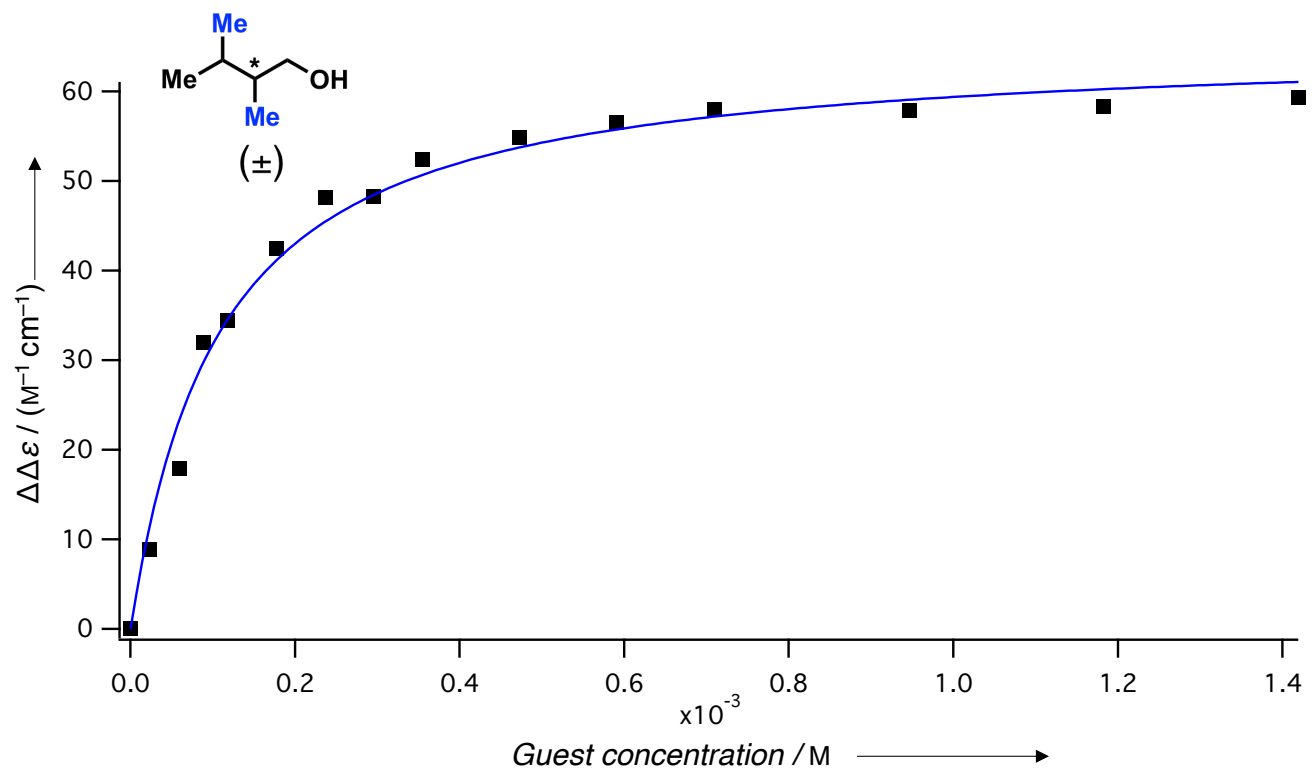

Figure S5. ECD binding isotherm of AAC $(P)_{4}-\mathbf{1}$ with $( \pm)$-2,3-dimethylbutan-1-ol $(R / S)-6$ in $n$-octane at $293 \mathrm{~K}$. $K_{\mathrm{a}}=9.88 \cdot 10^{3} \pm 0.90 \cdot 10^{3}, \Delta \Delta \varepsilon_{\max }=59.4 \mathrm{M}^{-1} \mathrm{~cm}^{-1}, \Delta \Delta \varepsilon_{\mathrm{sat}}=65.5 \pm 1.4 \mathrm{M}^{-1} \mathrm{~cm}^{-1}$.

\section{2,2,3-Trimethylbutan-1-ol 9}

Table S6. Experimental values for the determination of the association constant $K_{\mathrm{a}}$ of AAC $(P)_{4}-1$ with 2,2,3trimethylbutan-1-ol 9 in $n$-octane at $293 \mathrm{~K}$.

\begin{tabular}{ccccccc}
\hline $\begin{array}{c}K_{\mathrm{a}} \\
\mathrm{M}^{-1}\end{array}$ & $\begin{array}{c}\mathrm{AAC}(P)_{4}-\mathbf{1} \\
(\mu \mathrm{M})\end{array}$ & $\begin{array}{c}\text { [guest }]_{\max } \\
(\mathrm{mM})\end{array}$ & $\begin{array}{c}\text { guest } \\
\text { equiv.max }\end{array}$ & $\begin{array}{c}\Delta \Delta \varepsilon_{\max } \\
\left(\mathrm{M}^{-1} \mathrm{~cm}^{-1}\right)\end{array}$ & $\begin{array}{c}\Delta \Delta \varepsilon_{\text {sat }} \\
\left(\mathrm{M}^{-1} \mathrm{~cm}^{-1}\right)\end{array}$ \\
\hline $\mathrm{ECD}$ & $6.9 \cdot 10^{3}$ & 8.8 & 1.4 & 161.7 & 81.3 & 93.5 \\
\hline
\end{tabular}

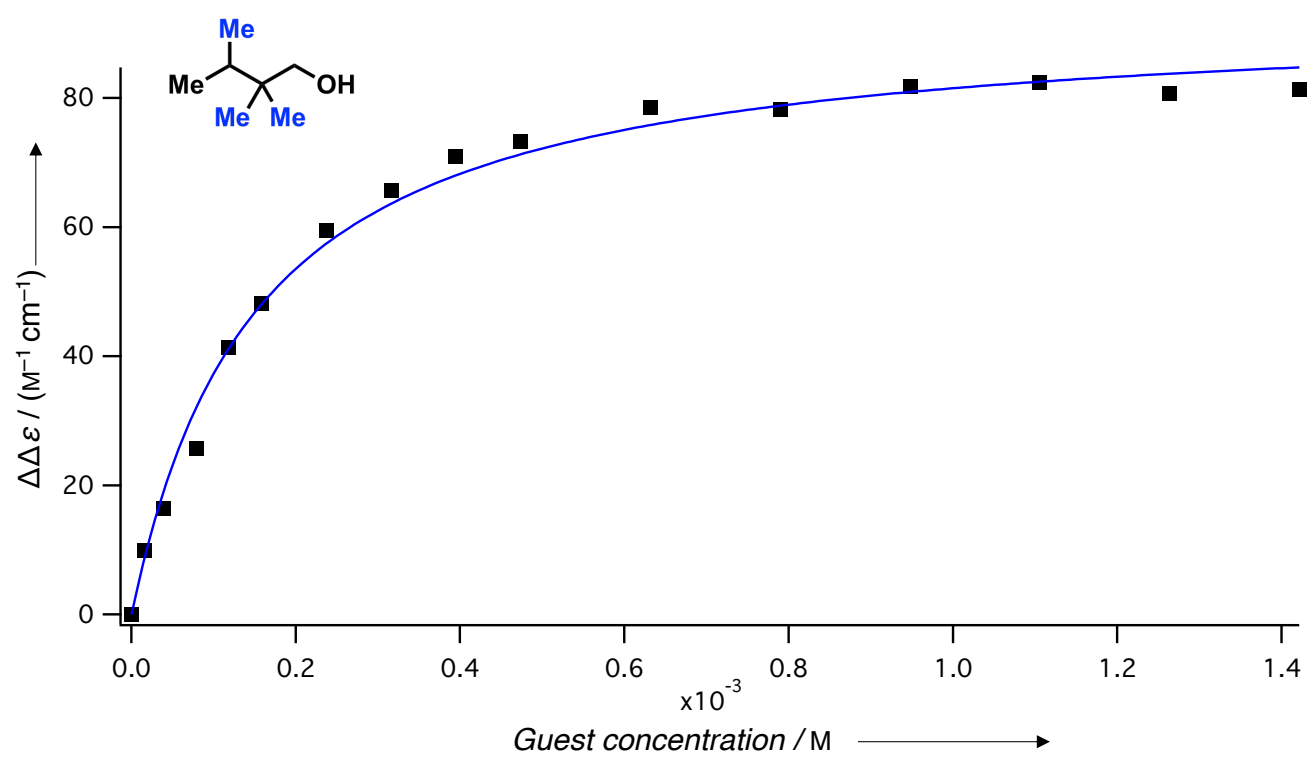

Figure S6. ECD binding isotherm of AAC $(P)_{4}-1$ with 2,2,3-trimethylbutan-1-ol 9 in $n$-octane at $293 \mathrm{~K} . K_{\mathrm{a}}=$ $6.88 \cdot 10^{3} \pm 0.54 \cdot 10^{3}, \Delta \Delta \varepsilon_{\max }=81.3 \mathrm{M}^{-1} \mathrm{~cm}^{-1}, \Delta \Delta \varepsilon_{\text {sat }}=93.5 \pm 1.9 \mathrm{M}^{-1} \mathrm{~cm}^{-1}$. 
( \pm )-(2S*,3R*)-2,3-Dichlorobutan-1-ol $\left(R^{*}, S^{*}\right)-7$

Table S7. Experimental values for the determination of the association constant $K_{\mathrm{a}}$ of AAC $(P)_{4}-\mathbf{1}$ with $( \pm)$ $\left(2 S^{*}, 3 R^{*}\right)$-2,3-dichlorobutan-1-ol $\left(R^{*}, S^{*}\right)-7$ in $n$-octane at $293 \mathrm{~K}$.

\begin{tabular}{ccccccc}
\hline $\begin{array}{c}K_{\mathrm{a}} \\
\mathrm{M}^{-1}\end{array}$ & $\begin{array}{c}\mathrm{AAC}(P)_{4}-\mathbf{1} \\
(\mu \mathrm{M})\end{array}$ & $\begin{array}{c}\text { [guest }]_{\max } \\
(\mathrm{mM})\end{array}$ & $\begin{array}{c}\text { guest } \\
\text { equiv.max }\end{array}$ & $\begin{array}{c}\Delta \Delta \varepsilon_{\max } \\
\left(\mathrm{M}^{-1} \mathrm{~cm}^{-1}\right)\end{array}$ & $\begin{array}{c}\Delta \Delta \varepsilon_{\text {sat }} \\
\left(\mathrm{M}^{-1} \mathrm{~cm}^{-1}\right)\end{array}$ \\
\hline $\mathrm{ECD}$ & $1.5 \cdot 10^{4}$ & 8.8 & 0.7 & 79.4 & 65.6 & 74.8 \\
\hline
\end{tabular}

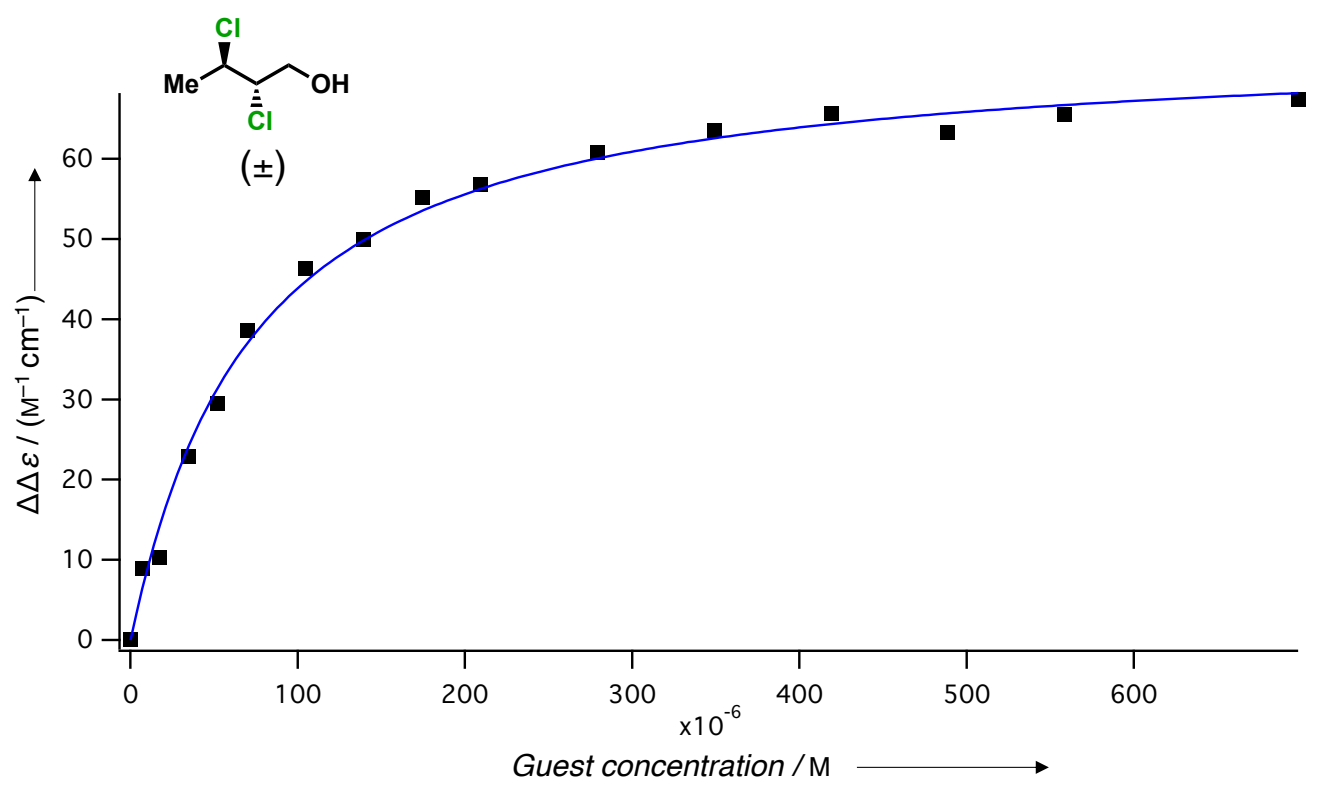

Figure S7. ECD binding isotherm of AAC $(P)_{4}-1$ with $( \pm)$ - $\left(2 S^{*}, 3 R^{*}\right)-2,3$-dichlorobutan-1-ol $\left(R^{*}, S^{*}\right)-7$ in $n$ octane at $293 \mathrm{~K} . K_{\mathrm{a}}=1.50 \cdot 10^{4} \pm 0.10 \cdot 10^{4}, \Delta \Delta \varepsilon_{\max }=65.6 \mathrm{M}^{-1} \mathrm{~cm}^{-1}, \Delta \Delta \varepsilon_{\text {sat }}=74.8 \pm 1.3 \mathrm{M}^{-1} \mathrm{~cm}^{-1}$.

\section{$( \pm)-\left(2 R^{*}, 3 R^{*}\right)-2,3-D i c h l o r o b u t a n-1-o l ~\left(R^{*}, R^{*}\right)-7$}

Table S8. Experimental values for the determination of the association constant $K_{\mathrm{a}}$ of AAC $(P)_{4}-\mathbf{1}$ with $( \pm)$ $\left(2 R^{*}, 3 R^{*}\right)$-2,3-dichlorobutan-1-ol $\left(R^{*}, R^{*}\right)-7$ in $n$-octane at $293 \mathrm{~K}$.

\begin{tabular}{|c|c|c|c|c|c|c|}
\hline & $\begin{array}{c}K_{\mathrm{a}} \\
\mathrm{M}^{-1}\end{array}$ & $\begin{array}{c}\text { AAC }(P)_{4-1} \\
(\mu \mathrm{M})\end{array}$ & $\begin{array}{c}\text { [guest] }]_{\max } \\
(\mathrm{mM})\end{array}$ & $\begin{array}{c}\text { guest } \\
\text { equiv.max }\end{array}$ & $\begin{array}{c}\Delta \Delta \varepsilon_{\max } \\
\left(\mathrm{M}^{-1} \mathrm{~cm}^{-1}\right)\end{array}$ & $\begin{array}{c}\Delta \Delta \varepsilon_{\mathrm{sat}} \\
\left(\mathrm{M}^{-1} \mathrm{~cm}^{-1}\right)\end{array}$ \\
\hline ECD & $7.0 \cdot 10^{4}$ & 8.8 & 0.7 & 46.4 & 82.3 & 86.7 \\
\hline
\end{tabular}




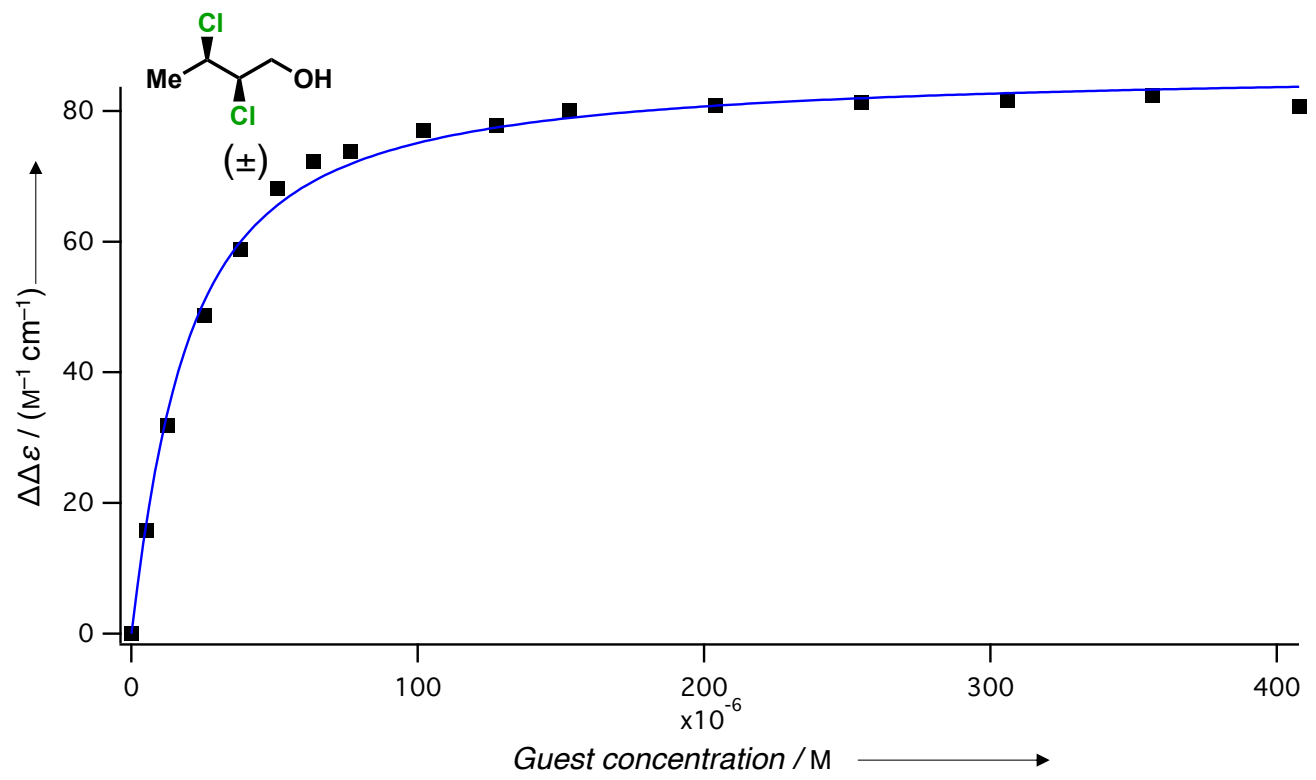

Figure S8. ECD binding isotherm of AAC $(P)_{4}-1$ with $( \pm)-\left(2 R^{*}, 3 R^{*}\right)-2,3$-dichlorobutan-1-ol $\left(R^{*}, R^{*}\right)-7$ in $n$ octane at $293 \mathrm{~K} . K_{\mathrm{a}}=7.02 \cdot 10^{4} \pm 0.46 \cdot 10^{4}, \Delta \Delta \varepsilon_{\max }=82.3 \mathrm{M}^{-1} \mathrm{~cm}^{-1}, \Delta \Delta \varepsilon_{\mathrm{sat}}=86.7 \pm 0.9 \mathrm{M}^{-1} \mathrm{~cm}^{-1}$.

\section{$( \pm)-\left(2 S^{*}, 3 R^{*}\right)-2,3-$ Dibromobutan-1-ol $\left(R^{*}, S^{*}\right)-8$}

Table S9. Experimental values for the determination of the association constant $K_{\mathrm{a}}$ of AAC $(P)_{4}-\mathbf{1}$ with $( \pm)$ $\left(2 S^{*}, 3 R^{*}\right)$-2,3-dibromobutan-1-ol $\left(R^{*}, S^{*}\right)-8$ in $n$-octane at $293 \mathrm{~K}$.

\begin{tabular}{ccccccc}
\hline$K_{\mathrm{a}}$ & $\mathrm{AAC}(P)_{4}-\mathbf{1}$ \\
$\mathrm{M}^{-1}$ & $(\mu \mathrm{M})$ & $\begin{array}{c}\text { [guest }]_{\max } \\
(\mathrm{mM})\end{array}$ & $\begin{array}{c}\text { guest } \\
\text { equiv.max }\end{array}$ & $\begin{array}{c}\Delta \Delta \varepsilon_{\max } \\
\left(\mathrm{M}^{-1} \mathrm{~cm}^{-1}\right)\end{array}$ & $\begin{array}{c}\Delta \Delta \varepsilon_{\text {sat }} \\
\left(\mathrm{M}^{-1} \mathrm{~cm}^{-1}\right)\end{array}$ \\
\hline ECD & $7.6 \cdot 10^{4}$ & 9.7 & 0.5 & 53.0 & 102.6 & 107.0 \\
\hline
\end{tabular}




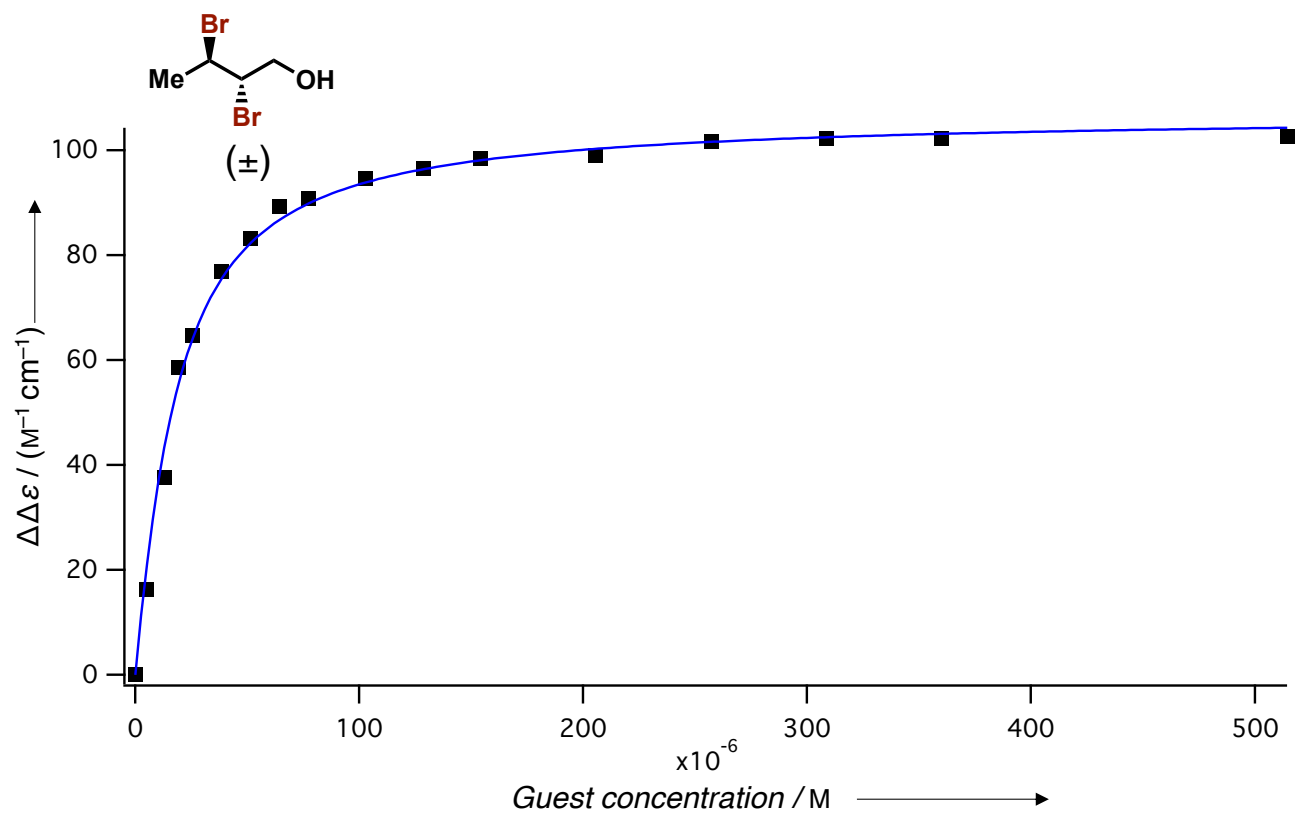

Figure S9. ECD binding isotherm of AAC $(P)_{4}-1$ with $( \pm)-\left(2 R^{*}, 3 R^{*}\right)-2,3$-dibromobutan-1-ol $\left(R^{*}, S^{*}\right)-8$ in $n$ octane at $293 \mathrm{~K} . K_{\mathrm{a}}=7.56 \cdot 10^{4} \pm 0.47 \cdot 10^{4}, \Delta \Delta \varepsilon_{\max }=102.6 \mathrm{M}^{-1} \mathrm{~cm}^{-1}, \Delta \Delta \varepsilon_{\text {sat }}=107.0 \pm 1.1 \mathrm{M}^{-1} \mathrm{~cm}^{-1}$.

\section{(-)-(2R,3S)-2,3-Dibromobutan-1-ol $(R, S)-8$}

Table S10. Experimental values for the determination of the association constant $K_{\mathrm{a}}$ of AAC $(P)_{4}-\mathbf{1}$ with (-)$(2 R, 3 S)$-2,3-dibromobutan-1-ol $(R, S)$-8 in $n$-octane at $293 \mathrm{~K}$.

\begin{tabular}{ccccccc}
\hline $\begin{array}{c}K_{\mathrm{a}} \\
\mathrm{M}^{-1}\end{array}$ & $\begin{array}{c}\mathrm{AAC}(P)_{4}-\mathbf{1} \\
(\mu \mathrm{M})\end{array}$ & $\begin{array}{c}\text { [guest }]_{\max } \\
(\mathrm{mM})\end{array}$ & $\begin{array}{c}\text { guest } \\
\text { equiv.max }\end{array}$ & $\begin{array}{c}\Delta \Delta \varepsilon_{\max } \\
\left(\mathrm{M}^{-1} \mathrm{~cm}^{-1}\right)\end{array}$ & $\begin{array}{c}\Delta \Delta \varepsilon_{\text {sat }} \\
\left(\mathrm{M}^{-1} \mathrm{~cm}^{-1}\right)\end{array}$ \\
\hline $\mathrm{ECD}$ & $6.1 \cdot 10^{4}$ & 9.5 & 0.5 & 36.1 & 80.6 & 86.3 \\
\hline
\end{tabular}

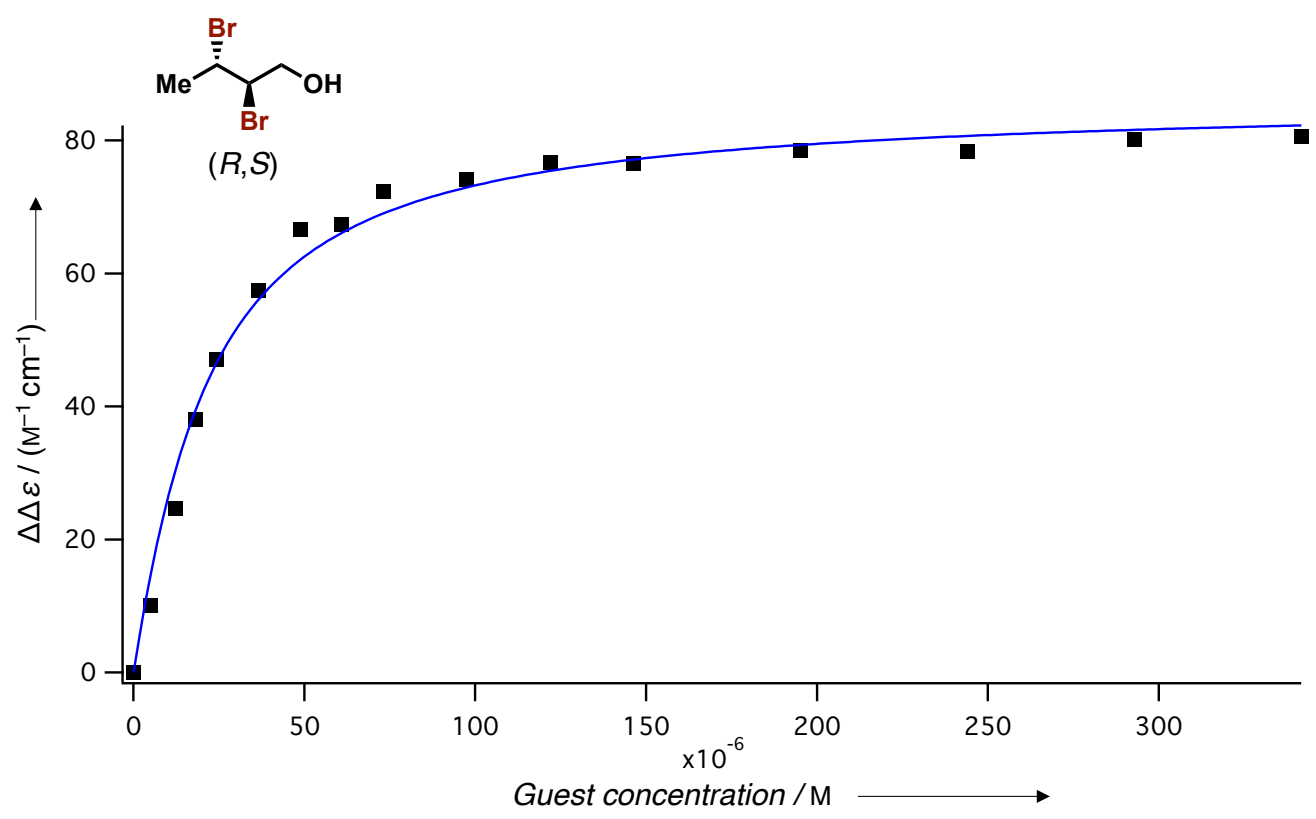

Figure S10. ECD binding isotherm of AAC $(P)_{4}-\mathbf{1}$ with (-)-(2R,3S)-2,3-dibromobutan-1-ol $(R, S)-\mathbf{8}$ in $n$-octane at $293 \mathrm{~K} . K_{\mathrm{a}}=6.1 \cdot 10^{4} \pm 0.56 \cdot 10^{4}, \Delta \Delta \varepsilon_{\max }=80.6 \mathrm{M}^{-1} \mathrm{~cm}^{-1}, \Delta \Delta \varepsilon_{\mathrm{sat}}=86.3 \pm 1.5 \mathrm{M}^{-1} \mathrm{~cm}^{-1}$. 


\section{(+)-(2S,3R)-2,3-Dibromobutan-1-ol $(S, R)-8$}

Table S11. Experimental values for the determination of the association constant $K_{\mathrm{a}}$ of AAC $(P)_{4}-\mathbf{1}$ with (+)$(2 S, 3 R)$-2,3-dibromobutan-1-ol $(S, R)-8$ in $n$-octane at $293 \mathrm{~K}$.

\begin{tabular}{ccccccc}
\hline $\begin{array}{c}K_{\mathrm{a}} \\
\mathrm{M}^{-1}\end{array}$ & $\begin{array}{c}\mathrm{AAC}(P)_{4}-\mathbf{1} \\
(\mu \mathrm{M})\end{array}$ & $\begin{array}{c}\text { [guest }]_{\max } \\
(\mathrm{mM})\end{array}$ & $\begin{array}{c}\text { guest } \\
\text { equiv.max }\end{array}$ & $\begin{array}{c}\Delta \Delta \varepsilon_{\max } \\
\left(\mathrm{M}^{-1} \mathrm{~cm}^{-1}\right)\end{array}$ & $\begin{array}{c}\Delta \Delta \varepsilon_{\text {sat }} \\
\left(\mathrm{M}^{-1} \mathrm{~cm}^{-1}\right)\end{array}$ \\
\hline $\mathrm{ECD}$ & $6.0 \cdot 10^{4}$ & 9.5 & 0.4 & 38.2 & 74.7 & 76.5 \\
\hline
\end{tabular}

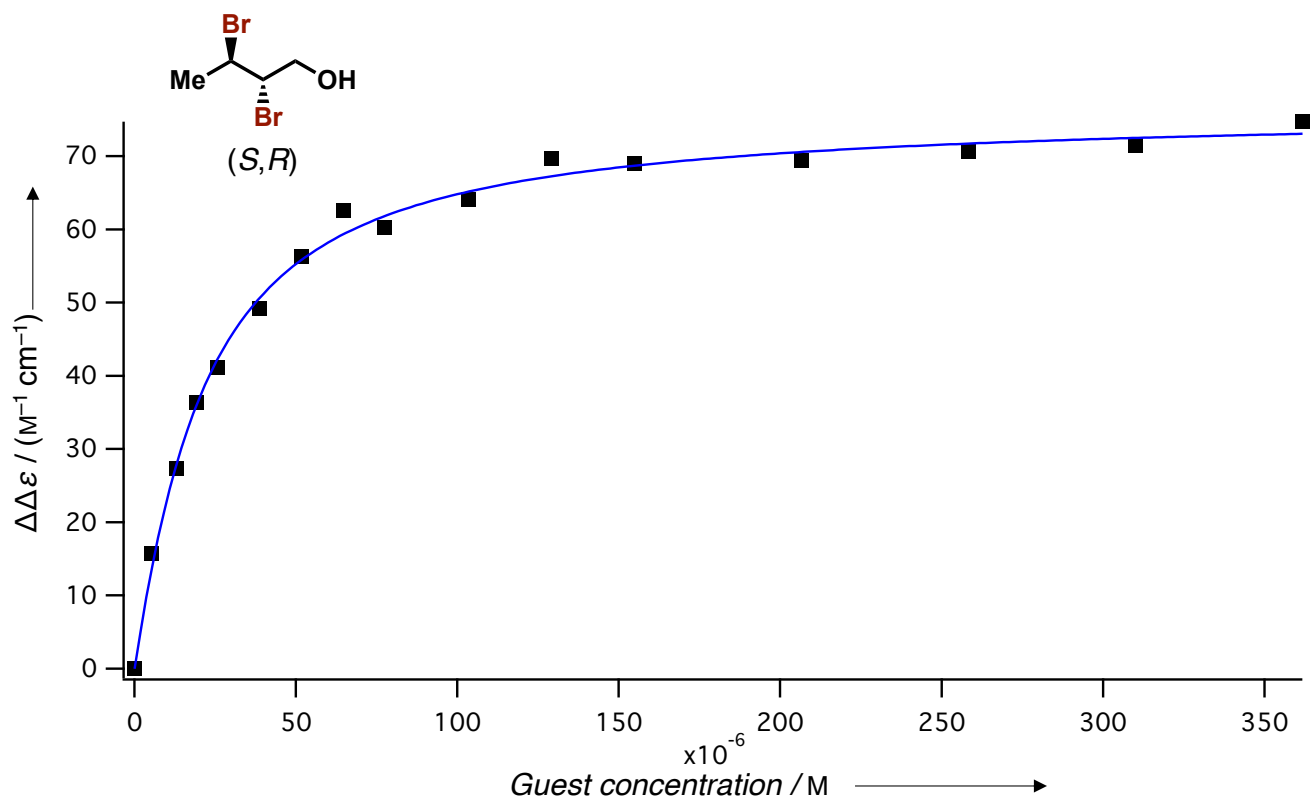

Figure S11. ECD binding isotherm of AAC $(P)_{4}-\mathbf{1}$ with $(+)-(2 S, 3 R)-2,3$-dibromobutan-1-ol $(S, R)$-8 in $n$-octane at $293 \mathrm{~K} . K_{\mathrm{a}}=6.04 \cdot 10^{4} \pm 0.37 \cdot 10^{4}, \Delta \Delta \varepsilon_{\max }=74.7 \mathrm{M}^{-1} \mathrm{~cm}^{-1}, \Delta \Delta \varepsilon_{\mathrm{sat}}=76.5 \pm 0.9 \mathrm{M}^{-1} \mathrm{~cm}^{-1}$.

\section{$( \pm)-\left(2 R^{*}, 3 R^{*}\right)-2,3-D i b r o m o b u t a n-1-o l ~\left(R^{*}, R^{*}\right)-8$}

Table S12. Experimental values for the determination of the association constant $K_{\mathrm{a}}$ of AAC $(P)_{4}-1$ with $( \pm)$ $\left(2 R^{*}, 3 R^{*}\right)$-2,3-dibromobutan-1-ol $\left(R^{*}, R^{*}\right)-8$ in $n$-octane at $293 \mathrm{~K}$.

\begin{tabular}{ccccccc}
\hline $\begin{array}{c}K_{\mathrm{a}} \\
\mathrm{M}^{-1}\end{array}$ & $\begin{array}{c}\mathrm{AAC}(P)_{4}-\mathbf{1} \\
(\mu \mathrm{M})\end{array}$ & $\begin{array}{c}\text { [guest }]_{\max } \\
(\mathrm{mM})\end{array}$ & $\begin{array}{c}\text { guest } \\
\text { equiv.max }\end{array}$ & $\begin{array}{c}\Delta \Delta \varepsilon_{\max } \\
\left(\mathrm{M}^{-1} \mathrm{~cm}^{-1}\right)\end{array}$ & $\begin{array}{c}\Delta \Delta \varepsilon_{\text {sat }} \\
\left(\mathrm{M}^{-1} \mathrm{~cm}^{-1}\right)\end{array}$ \\
\hline $\mathrm{ECD}$ & $3.8 \cdot 10^{5}$ & 8.6 & 0.2 & 24.0 & 88.7 & 89.4 \\
\hline
\end{tabular}




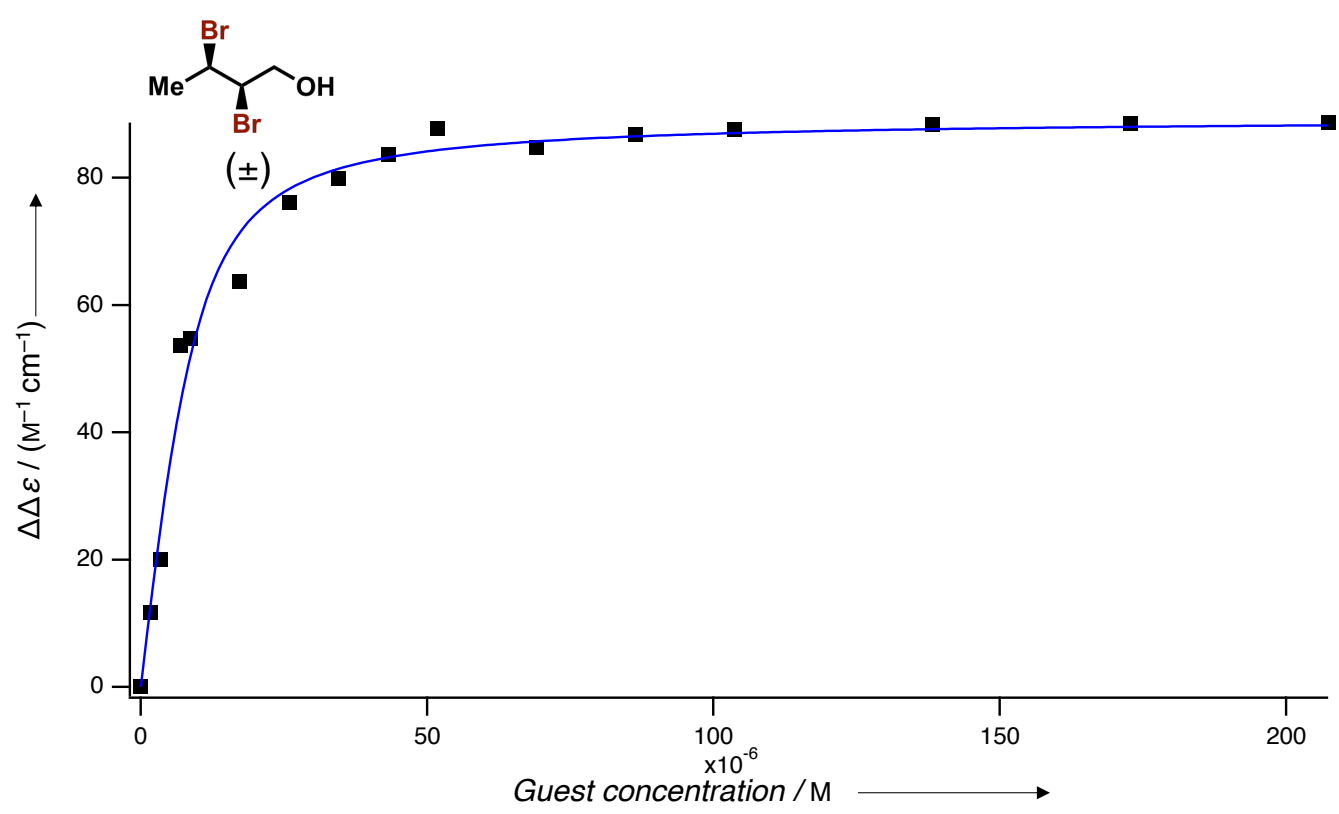

Figure S12. ECD binding isotherm of AAC $(P)_{4}-\mathbf{1}$ with $( \pm)-\left(2 R^{*}, 3 R^{*}\right)-2,3$-dibromobutan-1-ol $\left(R^{*}, R^{*}\right)-8$ in $n$ octane at $293 \mathrm{~K} . K_{\mathrm{a}}=3.82 \cdot 10^{5} \pm 0.73 \cdot 10^{5}, \Delta \Delta \varepsilon_{\max }=88.7 \mathrm{M}^{-1} \mathrm{~cm}^{-1}, \Delta \Delta \varepsilon_{\mathrm{sat}}=89.4 \pm 1.6 \mathrm{M}^{-1} \mathrm{~cm}^{-1}$.

\section{(+)-(2R,3R)-2,3-Dibromobutan-1-ol $(R, R)-8$}

Table S13. Experimental values for the determination of the association constant $K_{\mathrm{a}}$ of AAC $(P)_{4}-1$ with (+)$(2 R, 3 R)$-2,3-dibromobutan-1-ol $(R, R)-8$ in $n$-octane at $293 \mathrm{~K}$.

\begin{tabular}{ccccccc}
\hline & $\begin{array}{c}K_{\mathrm{a}} \\
\mathrm{M}^{-1}\end{array}$ & $\begin{array}{c}\mathrm{AAC}(P)_{4}-\mathbf{1} \\
(\mu \mathrm{M})\end{array}$ & $\begin{array}{c}{[\text { guest }]_{\max }} \\
(\mathrm{mM})\end{array}$ & $\begin{array}{c}\text { guest } \\
\text { equiv.max }\end{array}$ & $\begin{array}{c}\Delta \Delta \varepsilon_{\max } \\
\left(\mathrm{M}^{-1} \mathrm{~cm}^{-1}\right)\end{array}$ & $\begin{array}{c}\Delta \Delta \varepsilon_{\text {sat }} \\
\left(\mathrm{M}^{-1} \mathrm{~cm}^{-1}\right)\end{array}$ \\
\hline $\mathrm{ECD}$ & $1.2 \cdot 10^{6}$ & 9.0 & 0.04 & 4.0 & 81.5 & 83.0 \\
\hline
\end{tabular}




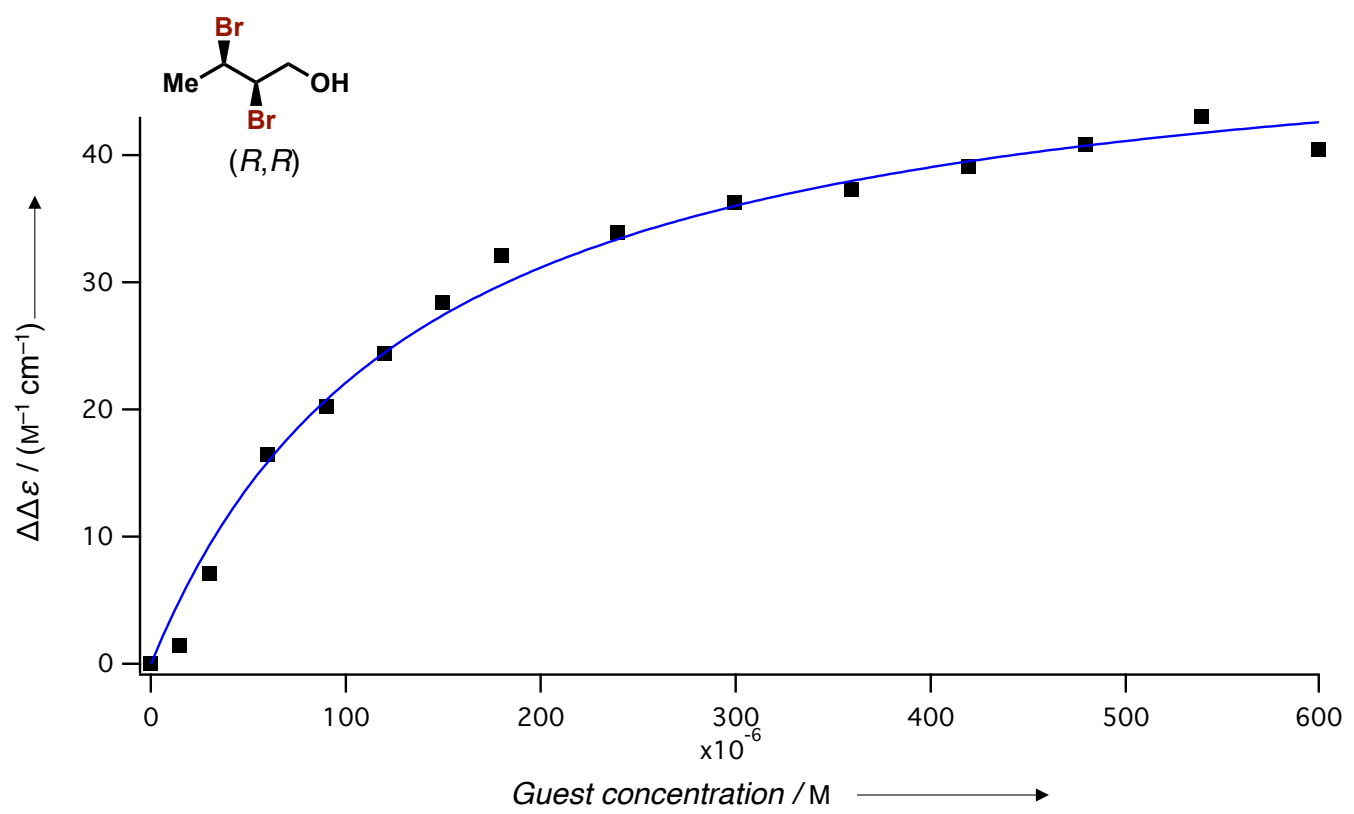

Figure S13. ECD binding isotherm of AAC $(P)_{4}-\mathbf{1}$ with $(+)-(2 R, 3 R)-2,3$-dibromobutan-1-ol $(R, R)-8$ in $n$-octane at $293 \mathrm{~K} . K_{\mathrm{a}}=1.17 \cdot 10^{6} \pm 0.18 \cdot 10^{6}, \Delta \Delta \varepsilon_{\max }=81.5 \mathrm{M}^{-1} \mathrm{~cm}^{-1}, \Delta \Delta \varepsilon_{\mathrm{sat}}=83.0 \pm 1.2 \mathrm{M}^{-1} \mathrm{~cm}^{-1}$.

\section{(-)-(2S,3S)-2,3-Dibromobutan-1-ol $(S, S)-8$}

Table S14. Experimental values for the determination of the association constant $K_{\mathrm{a}}$ of AAC $(P)_{4}-1$ with $(-)$ $(2 S, 3 S)$-2,3-dibromobutan-1-ol $(S, S)-8$ in $n$-octane at $293 \mathrm{~K}$.

\begin{tabular}{ccccccc}
\hline$K_{\mathrm{a}}$ & $\mathrm{AAC}(P)_{4-1}$ \\
$\mathrm{M}^{-1}$ & $(\mu \mathrm{M})$ & $\begin{array}{c}\text { [guest }]_{\max } \\
(\mathrm{mM})\end{array}$ & $\begin{array}{c}\text { guest } \\
\text { equiv.max }\end{array}$ & $\begin{array}{c}\Delta \Delta \varepsilon_{\max } \\
\left(\mathrm{M}^{-1} \mathrm{~cm}^{-1}\right)\end{array}$ & $\begin{array}{c}\Delta \Delta \varepsilon_{\text {sat }} \\
\left(\mathrm{M}^{-1} \mathrm{~cm}^{-1}\right)\end{array}$ \\
\hline $\mathrm{ECD}$ & $2.8 \cdot 10^{5}$ & 9.0 & 0.03 & 3.7 & 65.3 & 74.9 \\
\hline
\end{tabular}

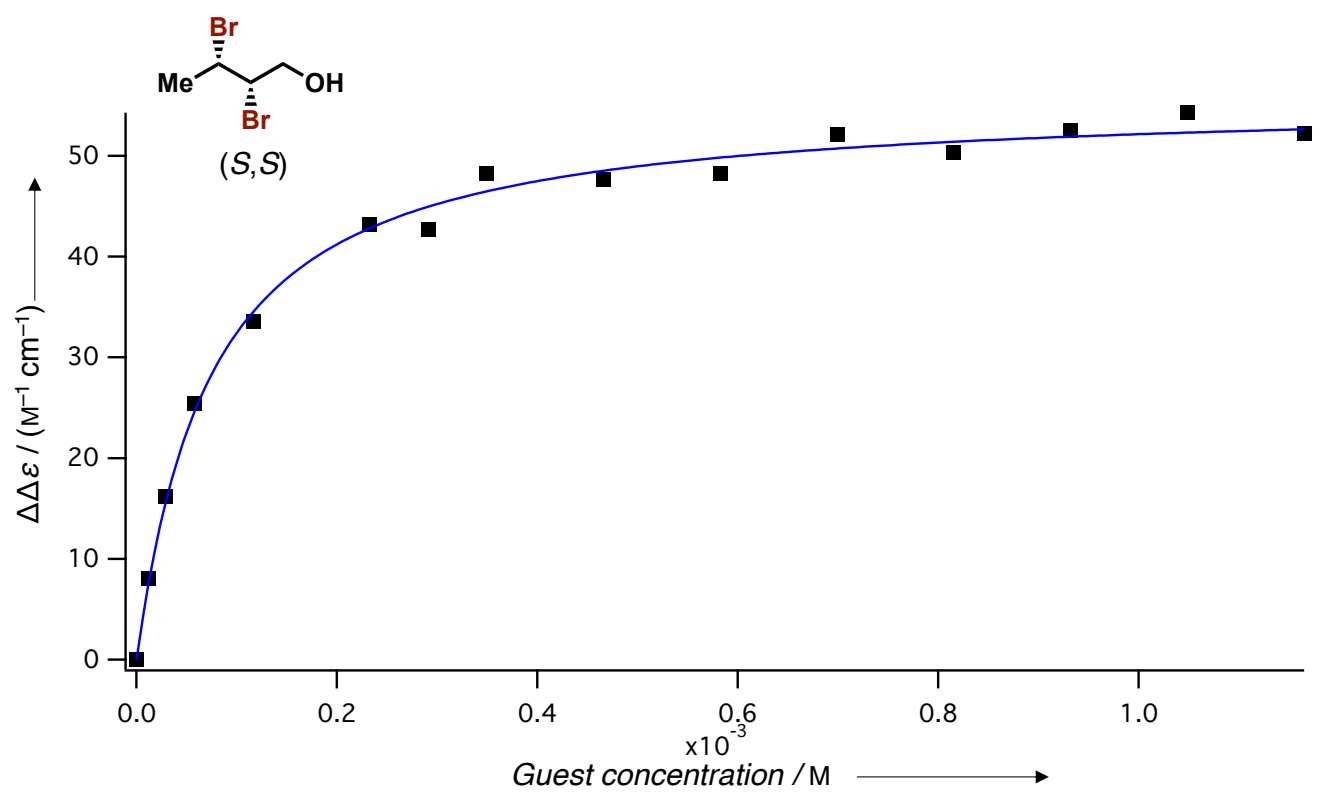

Figure S14. ECD binding isotherm of AAC $(P)_{4}-\mathbf{1}$ with (-)-(2S,3S)-2,3-dibromobutan-1-ol $(S, S)-8$ in $n$-octane at $293 \mathrm{~K} . K_{\mathrm{a}}=2.80 \cdot 10^{5} \pm 0.37 \cdot 10^{5}, \Delta \Delta \varepsilon_{\max }=65.3 \mathrm{M}^{-1} \mathrm{~cm}^{-1}, \Delta \Delta \varepsilon_{\mathrm{sat}}=74.9 \pm 2.0 \mathrm{M}^{-1} \mathrm{~cm}^{-1}$. 
$( \pm)-\left(2 S^{*}, 3 R^{*}\right)-2,3-D i b r o m o-2-m e t h y l b u t a n-1-o l ~\left(R^{*}, S^{*}\right)-10$

Table S15. Experimental values for the determination of the association constant $K_{\mathrm{a}}$ of AAC $(P)_{4}-1$ with $( \pm)$ $\left(2 S^{*}, 3 R^{*}\right)$-2,3-dibromo-2-methylbutan-1-ol $\left(R^{*}, S^{*}\right)$-10 in $n$-octane at $293 \mathrm{~K}$.

\begin{tabular}{ccccccc}
\hline & $\begin{array}{c}K_{\mathrm{a}} \\
\mathrm{M}^{-1}\end{array}$ & $\begin{array}{c}\mathrm{AAC}(P)_{4}-\mathbf{1} \\
(\mu \mathrm{M})\end{array}$ & $\begin{array}{c}\text { [guest }]_{\max } \\
(\mathrm{mM})\end{array}$ & $\begin{array}{c}\text { guest } \\
\text { equiv.max }\end{array}$ & $\begin{array}{c}\Delta \Delta \varepsilon_{\max } \\
\left(\mathrm{M}^{-1} \mathrm{~cm}^{-1}\right)\end{array}$ & $\begin{array}{c}\Delta \Delta \varepsilon_{\text {sat }} \\
\left(\mathrm{M}^{-1} \mathrm{~cm}^{-1}\right)\end{array}$ \\
\hline $\mathrm{ECD}$ & $1.2 \cdot 10^{5}$ & 9.7 & 0.03 & 26.2 & 87.2 & 92.2 \\
\hline
\end{tabular}

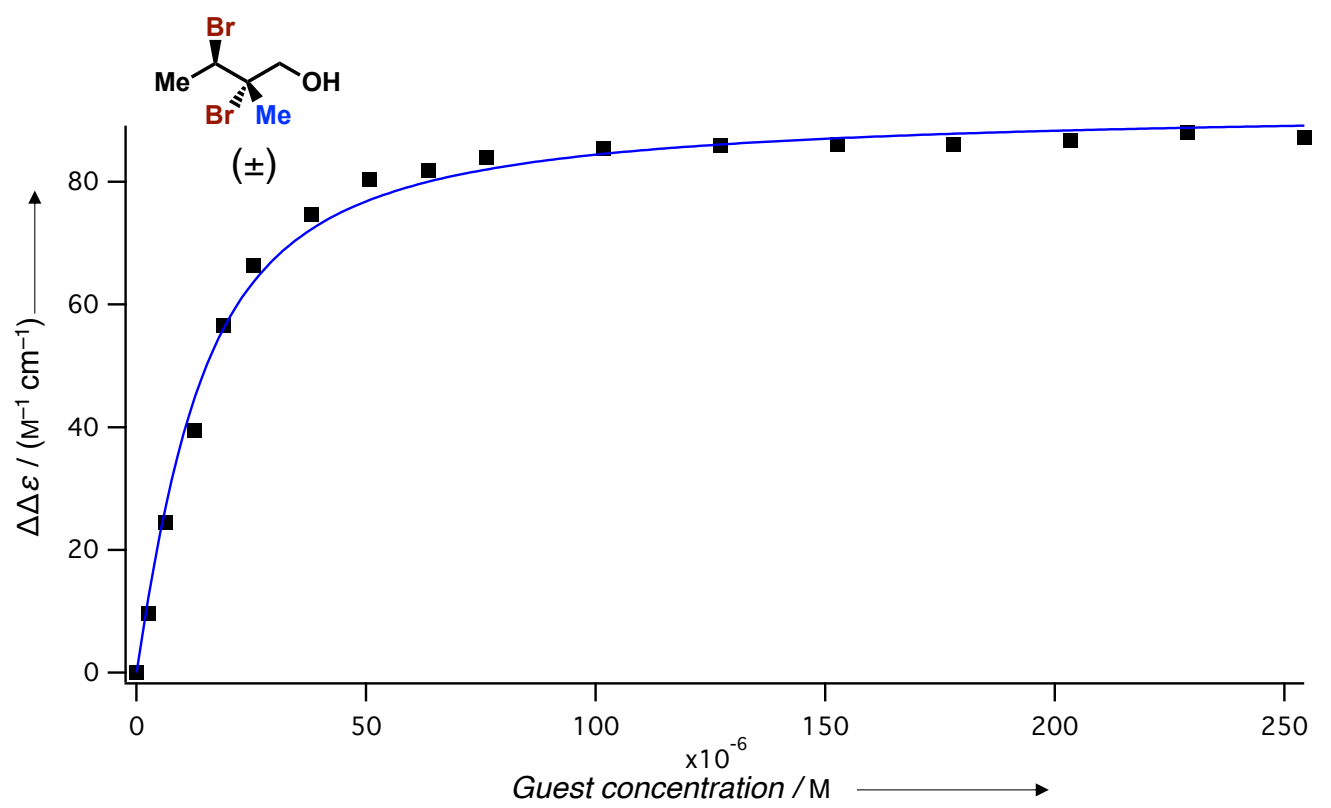

Figure S15. ECD binding isotherm of AAC $(P)_{4}-1$ with $( \pm)-\left(2 S^{*}, 3 R^{*}\right)$-2,3-dibromo-2-methylbutan-1-ol $\left(R^{*}, S^{*}\right)$ 10 in $n$-octane at $293 \mathrm{~K} . K_{\mathrm{a}}=1.19 \cdot 10^{5} \pm 0.10 \cdot 10^{5}, \Delta \Delta \varepsilon_{\max }=87.2 \mathrm{M}^{-1} \mathrm{~cm}^{-1}, \Delta \Delta \varepsilon_{\text {sat }}=92.2 \pm 1.1 \mathrm{M}^{-1} \mathrm{~cm}^{-1}$.

\section{$( \pm)-\left(2 R^{*}, 3 R^{*}\right)-2,3-D i b r o m o-2-m e t h y l b u t a n-1-o l ~\left(R^{*}, R^{*}\right)-10$}

Table S16. Experimental values for the determination of the association constant $K_{\mathrm{a}}$ of AAC $(P)_{4}-1$ with $( \pm)$ $\left(2 R^{*}, 3 R^{*}\right)$-2,3-dibromo-2-methylbutan-1-ol $\left(R^{*}, R^{*}\right)$-10 in $n$-octane at $293 \mathrm{~K}$.

\begin{tabular}{|c|c|c|c|c|c|c|}
\hline & $\begin{array}{c}K_{\mathrm{a}} \\
\mathrm{M}^{-1}\end{array}$ & $\begin{array}{c}\text { AAC }(P)_{4-1} \\
(\mu \mathrm{M})\end{array}$ & $\begin{array}{c}\text { [guest }]_{\max } \\
(\mathrm{mM})\end{array}$ & $\begin{array}{c}\text { guest } \\
\text { equiv.max }\end{array}$ & $\begin{array}{c}\Delta \Delta \varepsilon_{\max } \\
\left(\mathrm{M}^{-1} \mathrm{~cm}^{-1}\right)\end{array}$ & $\begin{array}{c}\Delta \Delta \varepsilon_{\mathrm{sat}} \\
\left(\mathrm{M}^{-1} \mathrm{~cm}^{-1}\right)\end{array}$ \\
\hline ECD & $3.8 \cdot 10^{5}$ & 4.9 & 0.02 & 4.8 & 94.9 & 92.3 \\
\hline
\end{tabular}




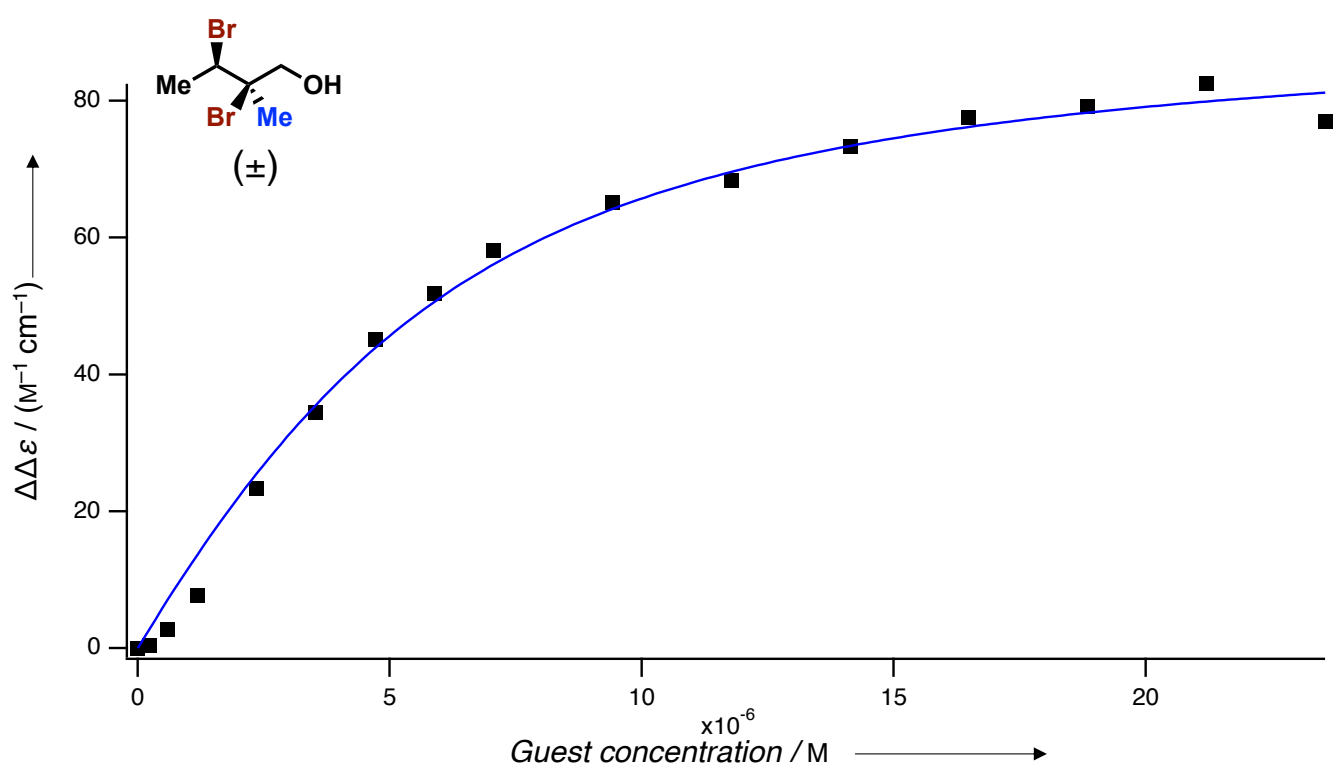

Figure S16. ECD binding isotherm of AAC $(P)_{4}-1$ with $( \pm)-\left(2 R^{*}, 3 R^{*}\right)$-2,3-dibromo-2-methylbutan-1-ol $\left(R^{*}, R^{*}\right)$ 10 in $n$-octane at $293 \mathrm{~K} . K_{\mathrm{a}}=3.79 \cdot 10^{5} \pm 0.59 \cdot 10^{5}, \Delta \Delta \varepsilon_{\max }=94.9 \mathrm{M}^{-1} \mathrm{~cm}^{-1}, \Delta \Delta \varepsilon_{\text {sat }}=92.3 \pm 3.1 \mathrm{M}^{-1} \mathrm{~cm}^{-1}$.

\section{(+)-(2R,3R)-2,3-Dibromo-2-methylbutan-1-ol $(R, R)-10$}

Table S17. Experimental values for the determination of the association constant $K_{\mathrm{a}}$ of AAC $(P)_{4}-1$ with (+)$(2 R, 3 R)$-2,3-dibromo-2-methylbutan-1-ol $(R, R)-10$ in $n$-octane at $293 \mathrm{~K}$.

\begin{tabular}{ccccccc}
\hline & $\begin{array}{c}K_{\mathrm{a}} \\
\mathrm{M}^{-1}\end{array}$ & $\begin{array}{c}\mathrm{AAC}(P)_{4}-\mathbf{1} \\
(\mu \mathrm{M})\end{array}$ & $\begin{array}{c}\text { [guest }]_{\max } \\
(\mathrm{mM})\end{array}$ & $\begin{array}{c}\text { guest } \\
\text { equiv.max }\end{array}$ & $\begin{array}{c}\Delta \Delta \varepsilon_{\max } \\
\left(\mathrm{M}^{-1} \mathrm{~cm}^{-1}\right)\end{array}$ & $\begin{array}{c}\Delta \Delta \varepsilon_{\text {sat }} \\
\left(\mathrm{M}^{-1} \mathrm{~cm}^{-1}\right)\end{array}$ \\
\hline $\mathrm{ECD}$ & $1.0 \cdot 10^{6}$ & 4.7 & 0.03 & 5.3 & 83.0 & 87.0 \\
\hline
\end{tabular}

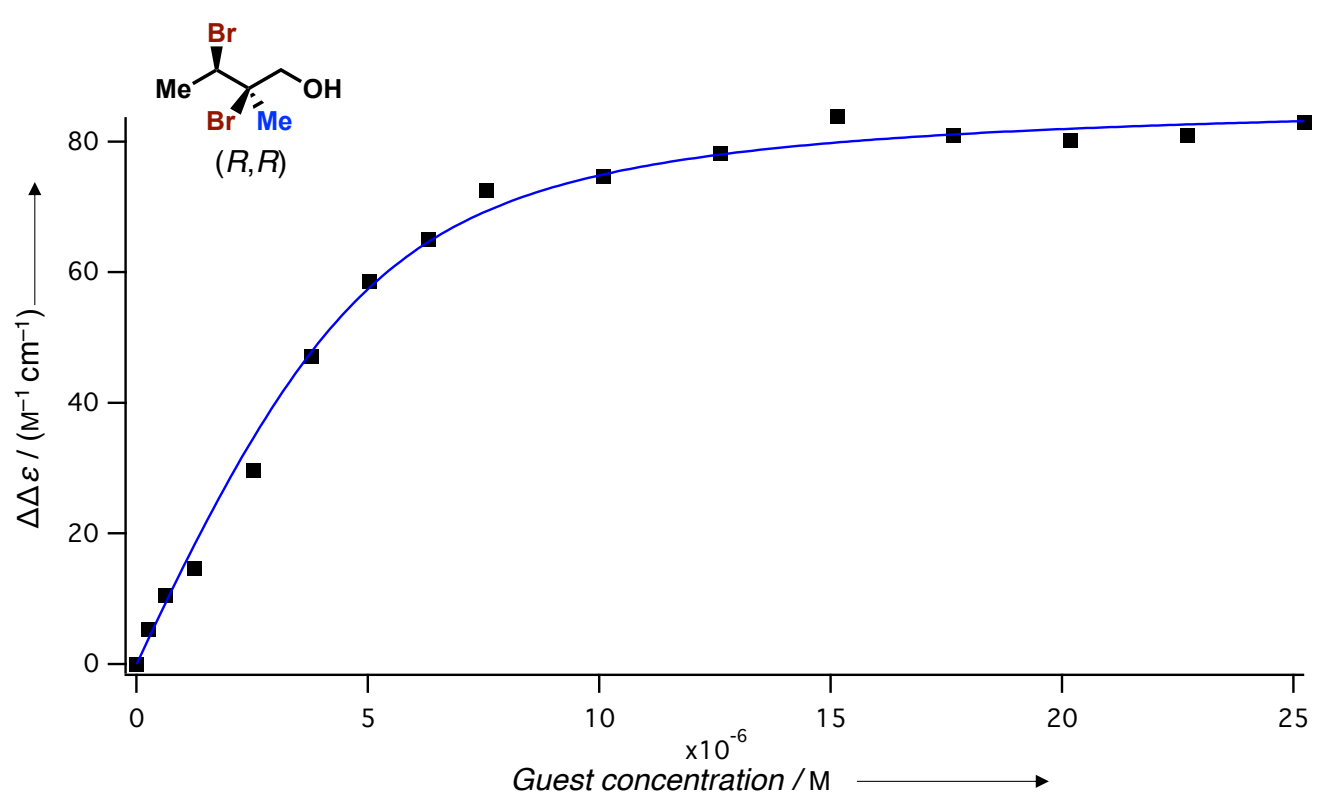

Figure S17. ECD binding isotherm of AAC $(P)_{4}-1$ with $(+)-(2 R, 3 R)-2,3$-dibromo-2-methylbutan-1 $(R, R)-10$ in $n$ octane at $293 \mathrm{~K} . K_{\mathrm{a}}=1.04 \cdot 10^{6} \pm 0.16 \cdot 10^{6}, \Delta \Delta \varepsilon_{\max }=83.0 \mathrm{M}^{-1} \mathrm{~cm}^{-1}, \Delta \Delta \varepsilon_{\text {sat }}=87.0 \pm 1.6 \mathrm{M}^{-1} \mathrm{~cm}^{-1}$. 
(-)-(2S,3S)-2,3-Dibromo-2-methylbutan-1-ol $(S, S)-10$

Table S18. Experimental values for the determination of the association constant $K_{\mathrm{a}}$ of AAC $(P)_{4}-1$ with (-)$(2 S, 3 S)$-2,3-dibromo-2-methylbutan-1-ol $(S, S)-\mathbf{1 0}$ in $n$-octane at $293 \mathrm{~K}$.

\begin{tabular}{ccccccc}
\hline & $\begin{array}{c}K_{\mathrm{a}} \\
\mathrm{M}^{-1}\end{array}$ & $\begin{array}{c}\mathrm{AAC}(P)_{4}-\mathbf{1} \\
(\mu \mathrm{M})\end{array}$ & $\begin{array}{c}\text { [guest }]_{\max } \\
(\mathrm{mM})\end{array}$ & $\begin{array}{c}\text { guest } \\
\text { equiv.max }\end{array}$ & $\begin{array}{c}\Delta \Delta \varepsilon_{\max } \\
\left(\mathrm{M}^{-1} \mathrm{~cm}^{-1}\right)\end{array}$ & $\begin{array}{c}\Delta \Delta \varepsilon_{\text {sat }} \\
\left(\mathrm{M}^{-1} \mathrm{~cm}^{-1}\right)\end{array}$ \\
\hline ECD & $1.3 \cdot 10^{6}$ & 9.6 & 0.1 & 13.6 & 64.3 & 64.0 \\
\hline
\end{tabular}

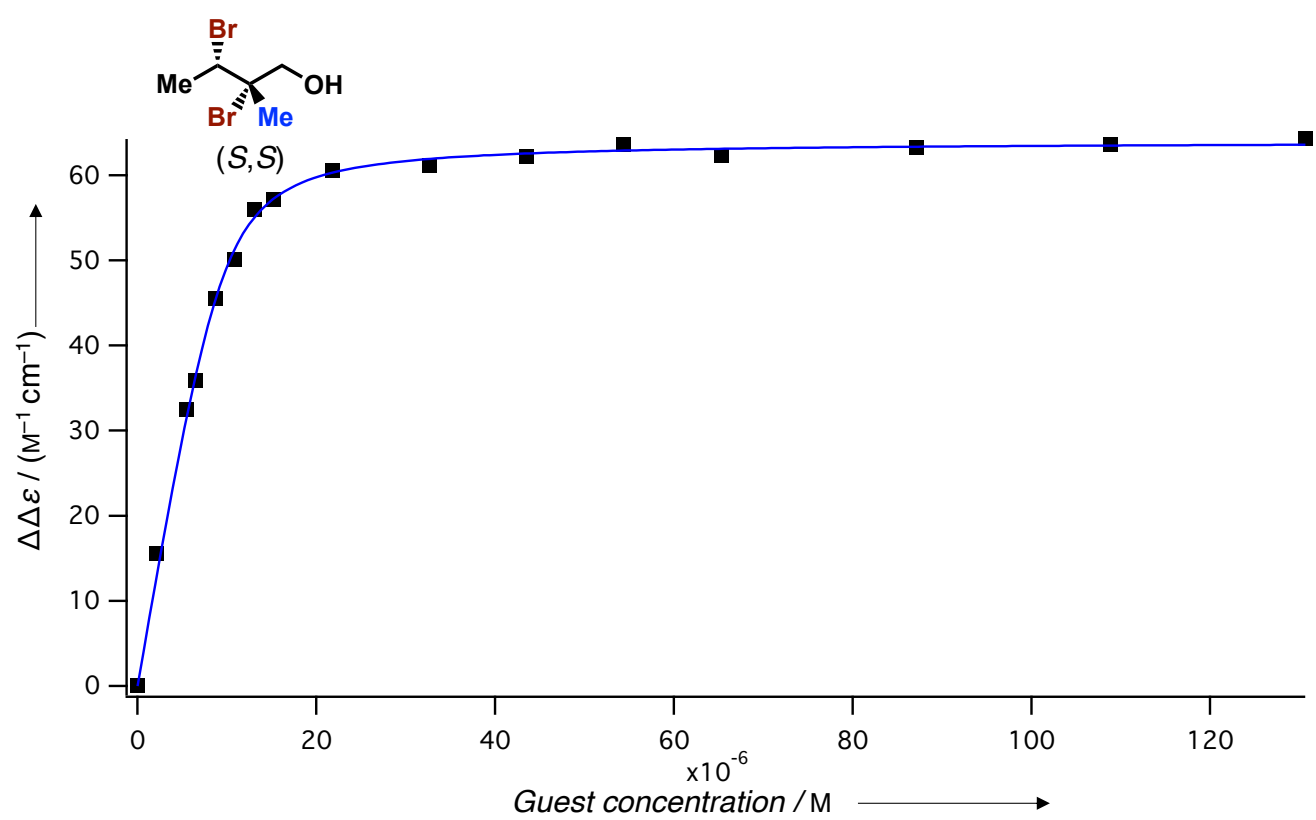

Figure S18. ECD binding isotherm of AAC $(P)_{4}-1$ with (-)-(2S,3S)-2,3-dibromo-2-methylbutan-1-ol $(S, S)$-10 in $n$-octane at $293 \mathrm{~K} . K_{\mathrm{a}}=1.28 \cdot 10^{6} \pm 0.12 \cdot 10^{6}, \Delta \Delta \varepsilon_{\max }=64.3 \mathrm{M}^{-1} \mathrm{~cm}^{-1}, \Delta \Delta \varepsilon_{\text {sat }}=64.0 \pm 0.4 \mathrm{M}^{-1} \mathrm{~cm}^{-1}$.

\section{( \pm )-(2S*,3R*)-2,3-Dibromo-4,4,4-trifluorobutan-1-ol $\left(R^{*}, S^{*}\right)-11$}

Table S19. Experimental values for the determination of the association constant $K_{\mathrm{a}}$ of AAC $(P)_{4}-1$ with $( \pm)$ $\left(2 S^{*}, 3 R^{*}\right)$-2,3-dibromo-4,4,4-trifluorobutan-1-ol $\left(R^{*}, S^{*}\right)-11$ in $n$-octane at $293 \mathrm{~K}$.

\begin{tabular}{ccccccc}
\hline $\begin{array}{c}K_{\mathrm{a}} \\
\mathrm{M}^{-1}\end{array}$ & $\begin{array}{c}\mathrm{AAC}(P)_{4}-\mathbf{1} \\
(\mu \mathrm{M})\end{array}$ & $\begin{array}{c}\text { [guest }]_{\max } \\
(\mathrm{mM})\end{array}$ & $\begin{array}{c}\text { guest } \\
\text { equiv.max }\end{array}$ & $\begin{array}{c}\Delta \Delta \varepsilon_{\max } \\
\left(\mathrm{M}^{-1} \mathrm{~cm}^{-1}\right)\end{array}$ & $\begin{array}{c}\Delta \Delta \varepsilon_{\text {sat }} \\
\left(\mathrm{M}^{-1} \mathrm{~cm}^{-1}\right)\end{array}$ \\
\hline ECD & $1.3 \cdot 10^{4}$ & 8.6 & 0.7 & 82.7 & 57.4 & 59.3 \\
\hline
\end{tabular}




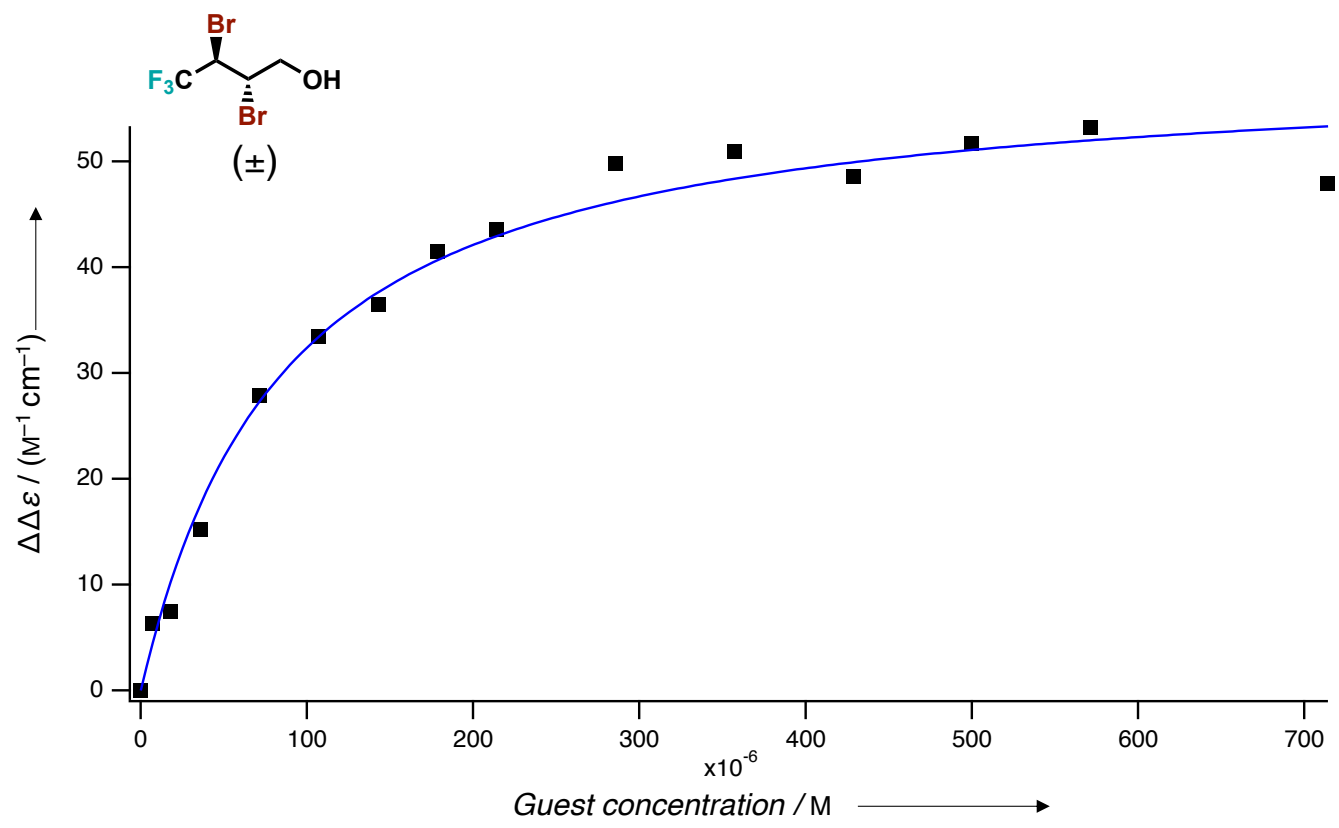

Figure S19. ECD binding isotherm of AAC $(P)_{4}-1$ with $( \pm)-\left(2 S^{*}, 3 R^{*}\right)$-2,3-dibromo-4,4,4-trifluorobutan-1-ol $\left(R^{*}, S^{*}\right)-11$ in $n$-octane at $293 \mathrm{~K} . K_{\mathrm{a}}=1.27 \cdot 10^{4} \pm 0.16 \cdot 10^{4}, \Delta \Delta \varepsilon_{\max }=57.4 \mathrm{M}^{-1} \mathrm{~cm}^{-1}, \Delta \Delta \varepsilon_{\text {sat }}=59.3 \pm 1.9 \mathrm{M}^{-1} \mathrm{~cm}^{-1}$.

\section{$( \pm)-\left(\left(2 R^{*}, 3 R^{*}\right)-2,3-D i b r o m o-4,4,4-t r i f l u o r o-2-m e t h y l b u t a n-1-o l ~\left(R^{*}, S^{*}\right)-12\right.$}

Table S20. Experimental values for the determination of the association constant $K_{\mathrm{a}}$ of AAC $(P)_{4}-\mathbf{1}$ with $( \pm)$ $\left(2 R^{*}, 3 R^{*}\right)$-2,3-Dibromo-4,4,4-trifluoro-2-methylbutan-1-ol $\left(R^{*}, S^{*}\right)$-12 in $n$-octane at $293 \mathrm{~K}$.

\begin{tabular}{ccccccc}
\hline$K_{\mathrm{a}}$ & $\mathrm{AAC}(P)_{4-1}$ \\
$\mathrm{M}^{-1}$ & $(\mu \mathrm{M})$ & $\begin{array}{c}\text { [guest }]_{\max } \\
(\mathrm{mM})\end{array}$ & $\begin{array}{c}\text { guest } \\
\text { equiv.max }\end{array}$ & $\begin{array}{c}\Delta \Delta \varepsilon_{\max } \\
\left(\mathrm{M}^{-1} \mathrm{~cm}^{-1}\right)\end{array}$ & $\begin{array}{c}\Delta \Delta \varepsilon_{\text {sat }} \\
\left(\mathrm{M}^{-1} \mathrm{~cm}^{-1}\right)\end{array}$ \\
\hline $\mathrm{ECD}$ & $1.2 \cdot 10^{4}$ & 9.1 & 0.8 & 87.7 & 71.1 & 78.5 \\
\hline
\end{tabular}

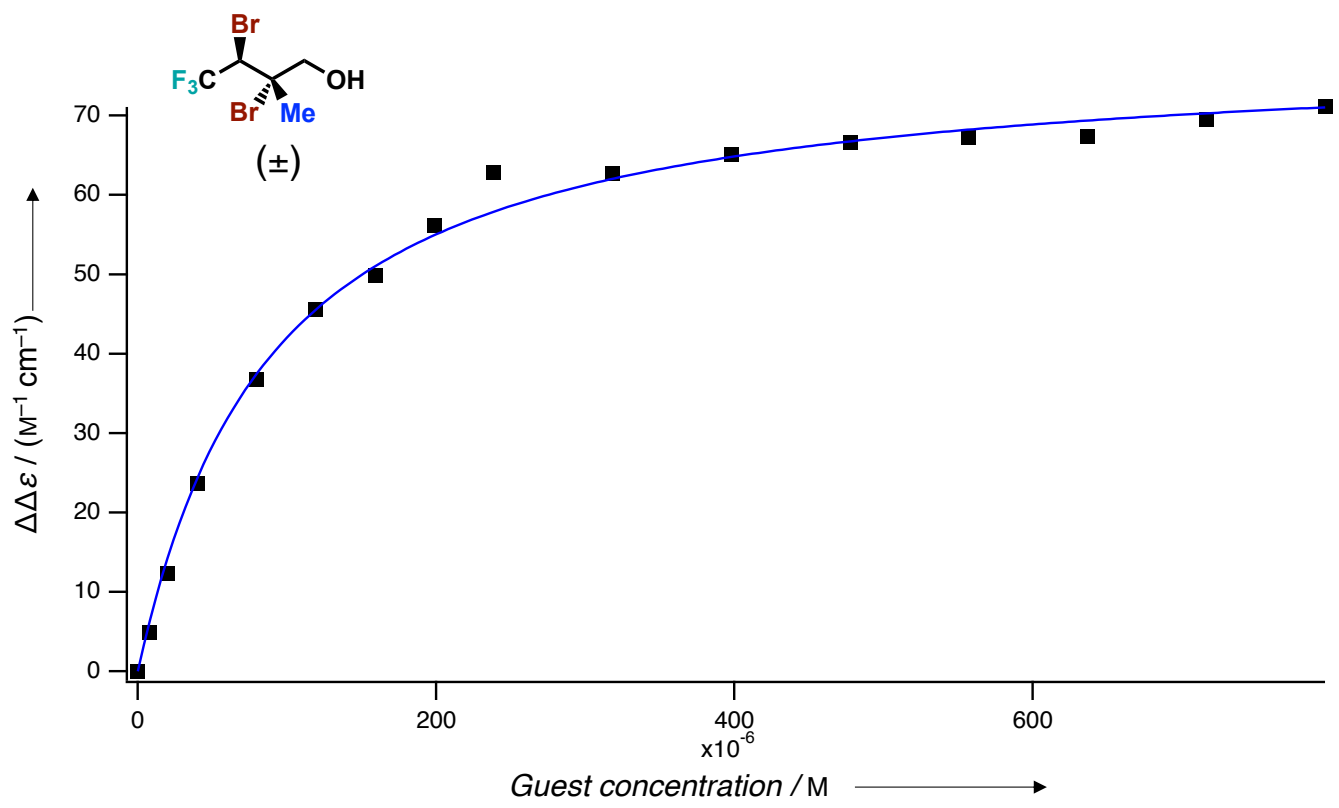

Figure S20. ECD binding isotherm of $\mathrm{AAC}(P)_{4}-1$ with $( \pm)-\left(2 R^{*}, 3 R^{*}\right)-2,3-$-Dibromo-4,4,4-trifluoro-2methylbutan-1-ol in $\left(R^{*}, S^{*}\right)-\mathbf{1 2} n$-octane at $293 \mathrm{~K} . K_{\mathrm{a}}=1.21 \cdot 10^{4} \pm 0.08 \cdot 10^{4}, \Delta \Delta \varepsilon$ max $=71.1 \mathrm{M}^{-1} \mathrm{~cm}^{-1}, \Delta \Delta \varepsilon_{\text {sat }}=78.5$ $\pm 1.2 \mathrm{M}^{-1} \mathrm{~cm}^{-1}$. 


\section{S5. Titration Data and Binding Isotherms Obtained by ITC}

\section{3-Methylbutan-1-ol 3}

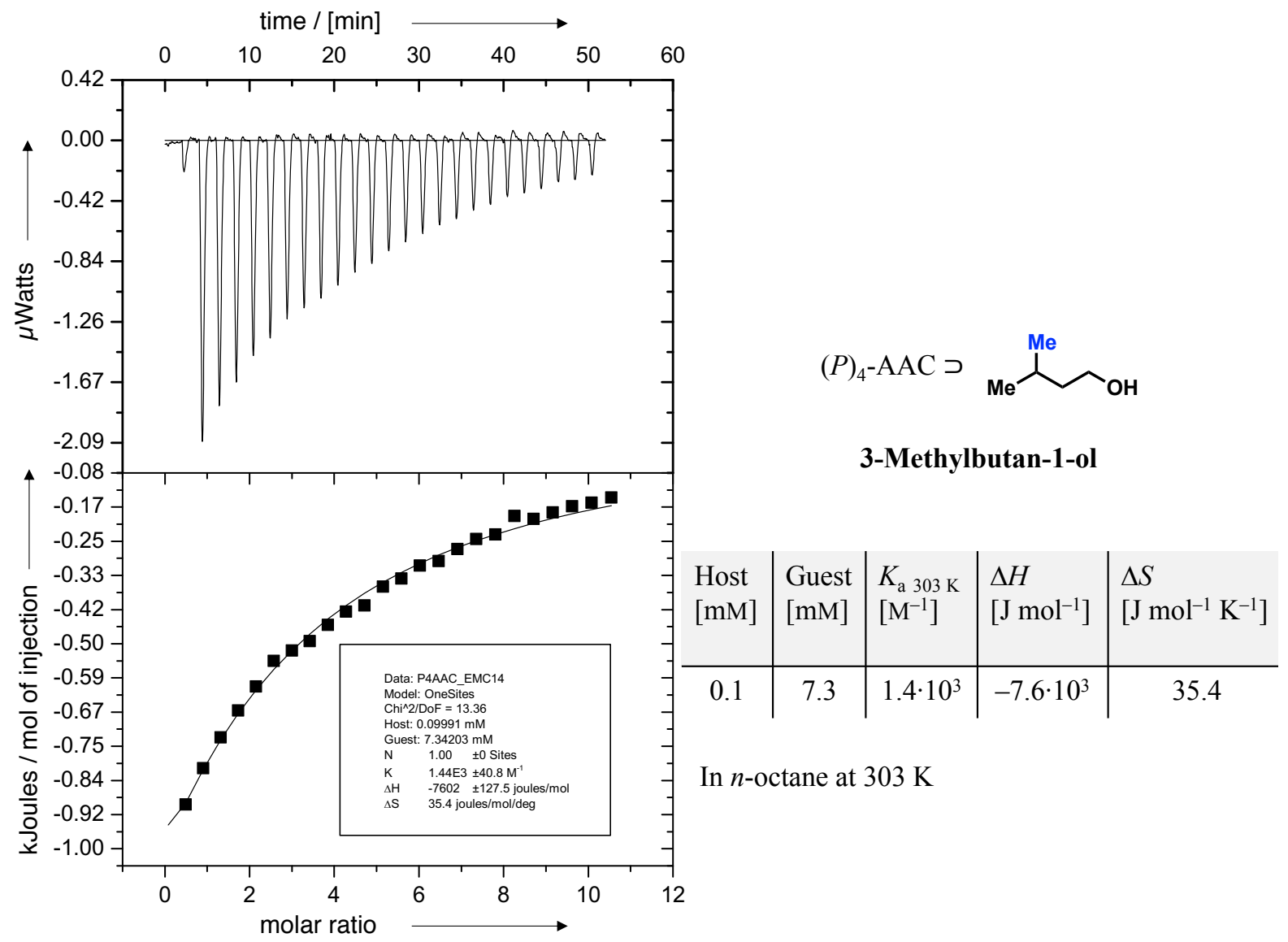

Figure S21. ITC binding isotherm of AAC $(P)_{4}-1$ with 3-methylbutan-1-ol 3 in $n$-octane at $303 \mathrm{~K}$. $K_{\mathrm{a}}=1.44 \cdot 10^{3} \pm 0.04 \cdot 10^{3} \mathrm{M}^{-1}$.

Table S21. Experimental values obtained from ITC titrations of AAC $(P)_{4-1}$ with 3-methylbutan-1-ol 3 in $n$ octane calculated for $293 \mathrm{~K}$.

\begin{tabular}{cccc}
\hline $\begin{array}{c}K_{\mathrm{a}} \\
\mathrm{M}^{-1}\end{array}$ & $\begin{array}{c}\Delta G_{293 \mathrm{~K}} \\
{\left[\mathrm{kcal} \mathrm{mol}^{-1}\right]}\end{array}$ & $\begin{array}{c}\Delta H \\
{\left[\mathrm{kcal} \mathrm{mol}^{-1}\right]}\end{array}$ & $\begin{array}{c}-\mathrm{T} \Delta S_{293 \mathrm{~K}} \\
{\left[\mathrm{kcal} \mathrm{mol}^{-1}\right]}\end{array}$ \\
\hline $1.6 \cdot 10^{3}$ & -4.3 & -1.8 & -2.5 \\
\hline
\end{tabular}


( \pm )-2-Methylbutan-1-ol $(R / S)-4$

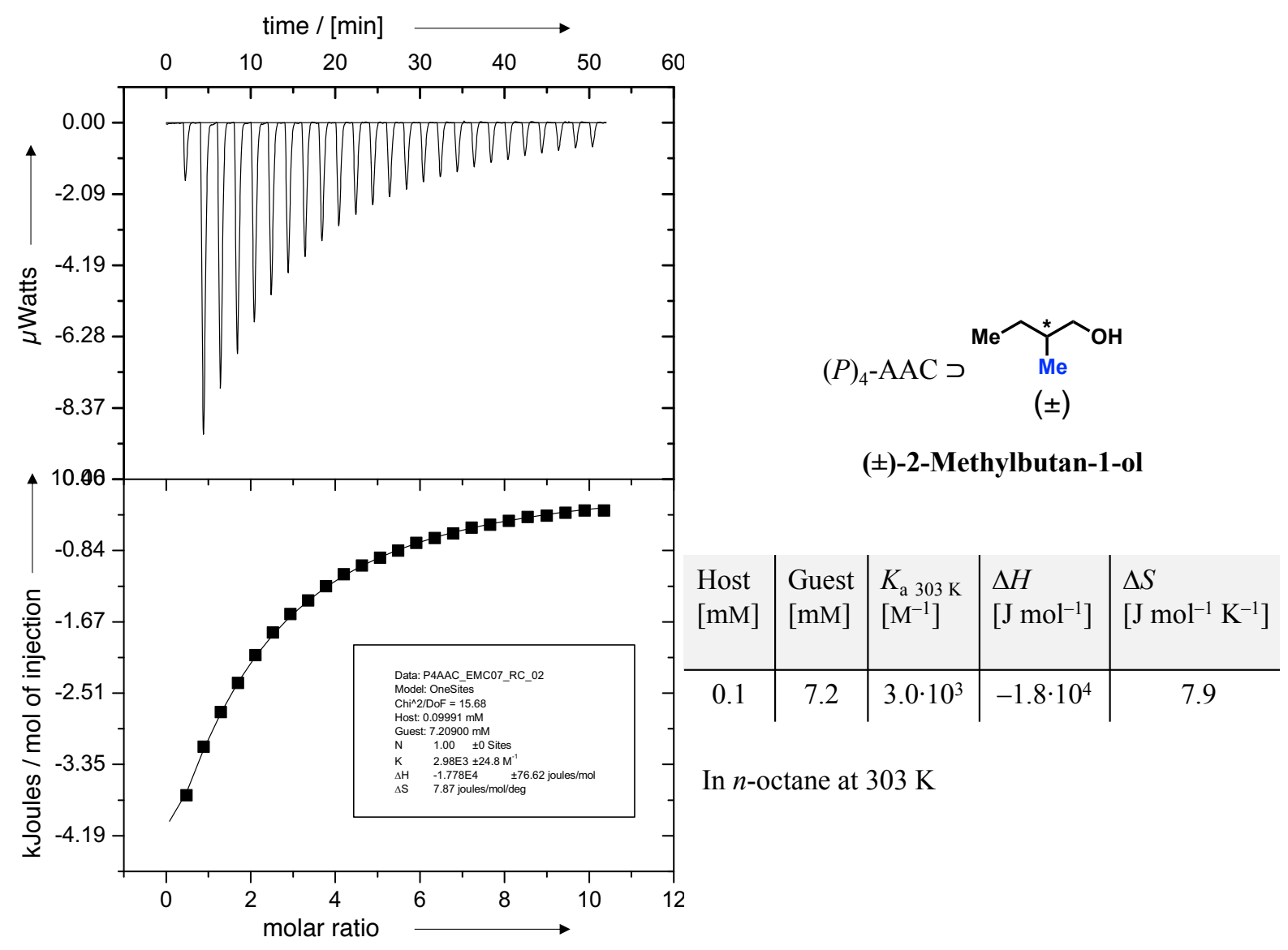

Figure S22. ITC binding isotherm of AAC $(P)_{4}-1$ with ( \pm )-2-methylbutan-1-ol $(R / S)$-4 in $n$-octane at $303 \mathrm{~K}$. $K_{\mathrm{a}}=2.98 \cdot 10^{3} \pm 0.03 \cdot 10^{3} \mathrm{M}^{-1}$.

Table S22. Experimental values obtained from ITC titrations of AAC $(P)_{4}-\mathbf{1}$ with $( \pm)$-2-methylbutan-1-ol $(R / S)$ 4 in $n$-octane calculated for $293 \mathrm{~K}$.

\begin{tabular}{cccc}
\hline $\begin{array}{c}K_{\mathrm{a}} \\
\mathrm{M}^{-1}\end{array}$ & $\begin{array}{c}\Delta G_{293 \mathrm{~K}} \\
{\left[\mathrm{kcal} \mathrm{mol}^{-1}\right]}\end{array}$ & $\begin{array}{c}\Delta H \\
{\left[\mathrm{kcal} \mathrm{mol}^{-1}\right]}\end{array}$ & $\begin{array}{c}-\mathrm{T} \Delta S_{293 \mathrm{~K}} \\
{\left[\mathrm{kcal} \mathrm{mol}^{-1}\right]}\end{array}$ \\
\hline $4.6 \cdot 10^{3}$ & -4.90 & -4.3 & -0.6 \\
\hline
\end{tabular}




\section{2,2-Dimethylbutan-1-ol 5}

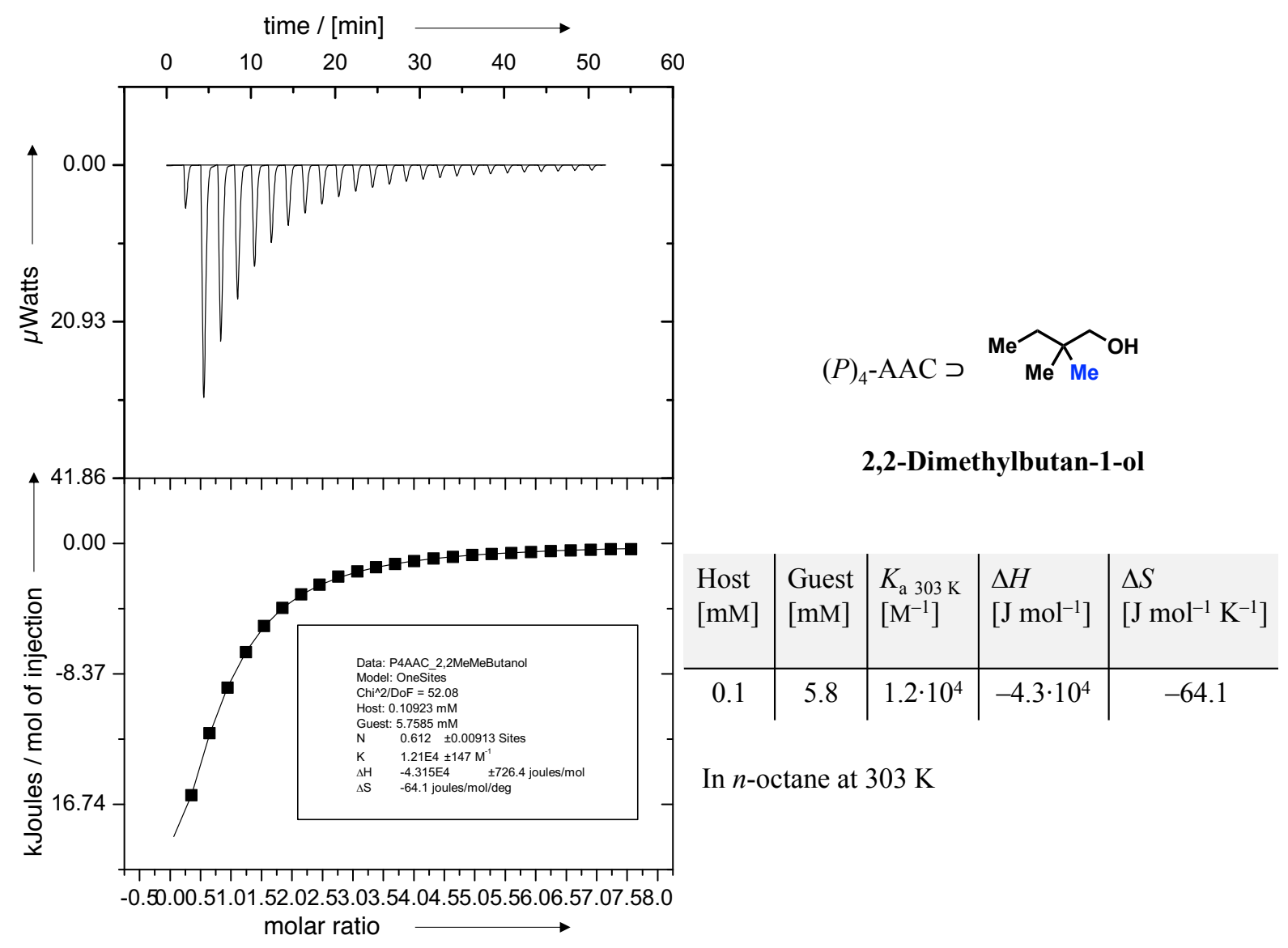

Figure S23. ITC binding isotherm of AAC $(P)_{4}-\mathbf{1}$ with 2,2-dimethylbutan-1-ol $\mathbf{5}$ in $n$-octane at $303 \mathrm{~K}$. $K_{\mathrm{a}}=1.21 \cdot 10^{4} \pm 0.02 \cdot 10^{4} \mathrm{M}^{-1}$.

Table S23. Experimental values obtained from ITC titrations of AAC $(P)_{4}$-1 with 2,2-dimethylbutan-1-ol $\mathbf{5}$ in $n$ octane calculated for $293 \mathrm{~K}$.

\begin{tabular}{cccc}
\hline $\begin{array}{c}K_{\mathrm{a}} \\
\mathrm{M}^{-1}\end{array}$ & $\begin{array}{c}\Delta G_{293 \mathrm{~K}} \\
{\left[\mathrm{kcal} \mathrm{mol}^{-1}\right]}\end{array}$ & $\begin{array}{c}\Delta H \\
{\left[\mathrm{kcal} \mathrm{mol}^{-1}\right]}\end{array}$ & $\begin{array}{c}-\mathrm{T} \Delta S_{293 \mathrm{~K}} \\
{\left[\mathrm{kcal} \mathrm{mol}^{-1}\right]}\end{array}$ \\
\hline $1.7 \cdot 10^{4}$ & -5.8 & -10.3 & +4.5 \\
\hline
\end{tabular}


( \pm )-2,3-Methylbutan-1-ol $(R / S)-6$

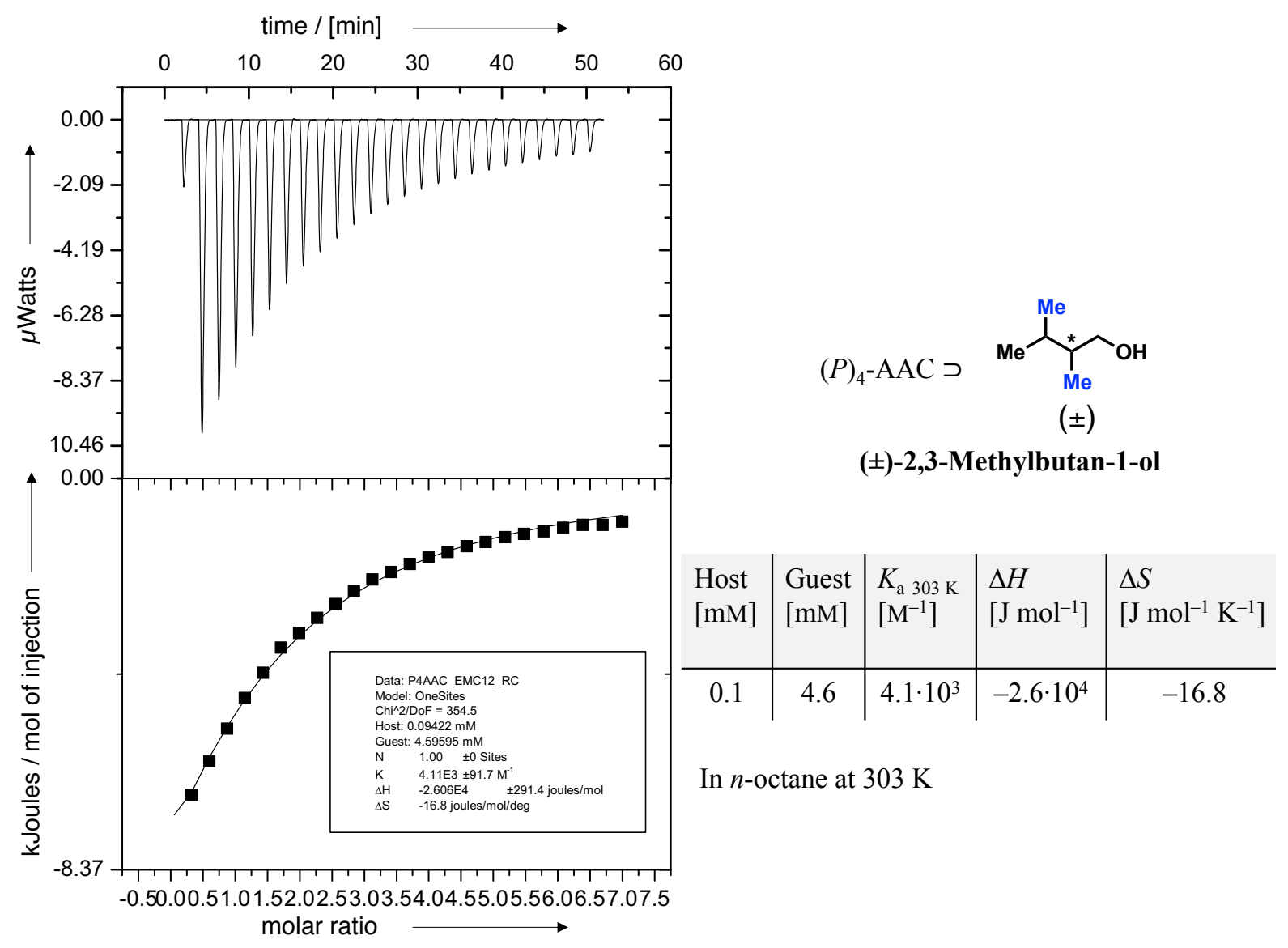

Figure S24. ITC binding isotherm of AAC $(P)_{4}-\mathbf{1}$ with ( \pm )-2,3-methylbutan-1-ol $(R / S)$-6 in $n$-octane at $303 \mathrm{~K}$. $K_{\mathrm{a}}=4.11 \cdot 10^{3} \pm 0.09 \cdot 10^{3} \mathrm{M}^{-1}$.

Table S24. Experimental values obtained from ITC titrations of AAC $(P)_{4}-\mathbf{1}$ with $( \pm)$-2,3-Methylbutan-1-ol $(R / S)$ 6 in $n$-octane calculated for $293 \mathrm{~K}$.

\begin{tabular}{cccc}
\hline $\begin{array}{c}K_{\mathrm{a}} \\
\mathrm{M}^{-1}\end{array}$ & $\begin{array}{c}\Delta G_{293 \mathrm{~K}} \\
{\left[\mathrm{kcal} \mathrm{mol}^{-1}\right]}\end{array}$ & $\begin{array}{c}\Delta H \\
{\left[\mathrm{kcal} \mathrm{mol}^{-1}\right]}\end{array}$ & $\begin{array}{c}-\mathrm{T} \Delta S_{293 \mathrm{~K}} \\
{\left[\mathrm{kcal} \mathrm{mol}^{-1}\right]}\end{array}$ \\
\hline $5.9 \cdot 10^{3}$ & -5.1 & -6.2 & +1.1 \\
\hline
\end{tabular}




\section{2,2,3-Trimethylbutan-1-ol 9}

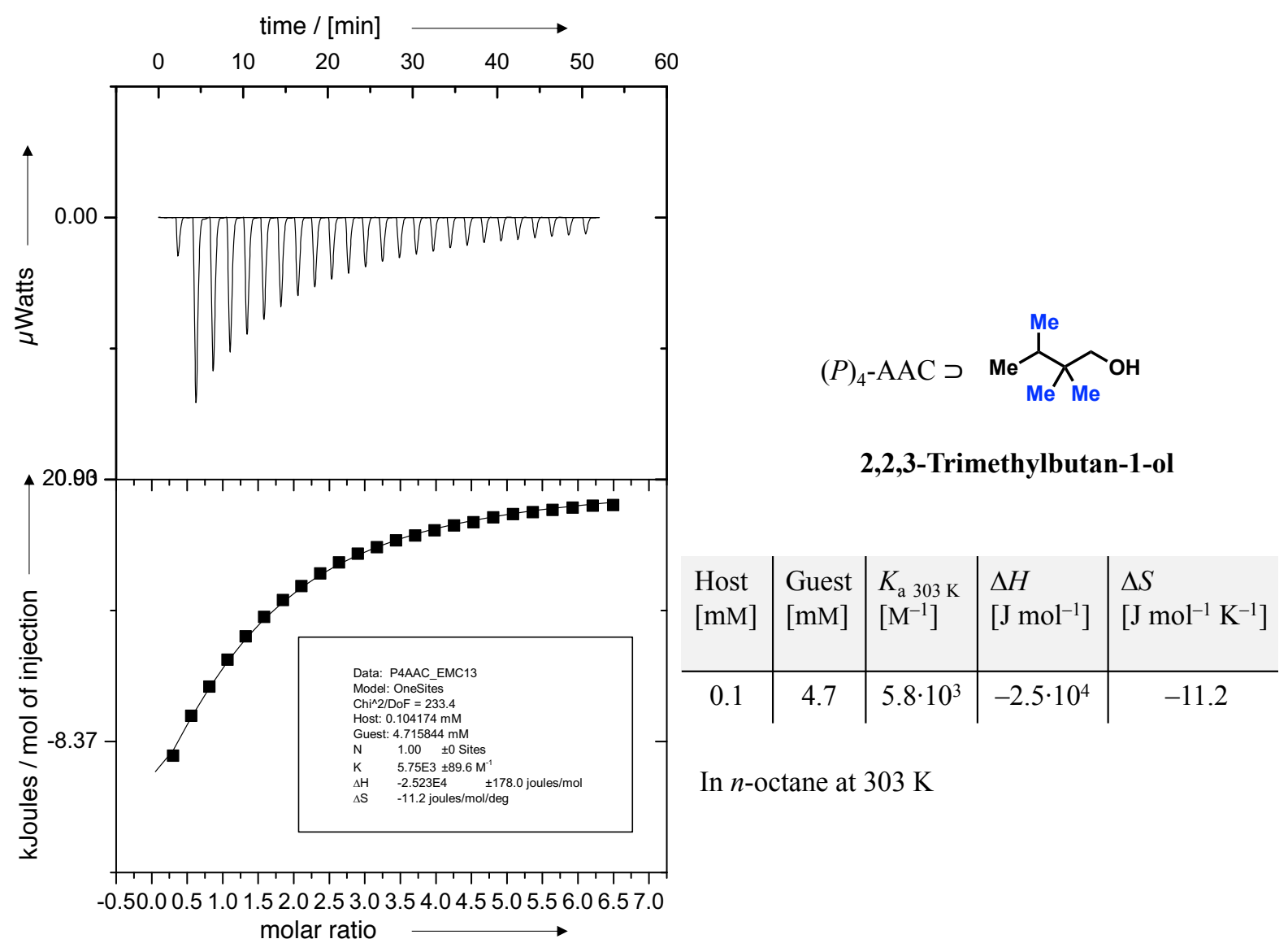

Figure S25. ITC binding isotherm of AAC $(P)_{4}-\mathbf{1}$ with 2,2,3-trimethylbutan-1-ol 9 in $n$-octane at $303 \mathrm{~K}$. $K_{\mathrm{a}}=5.75 \cdot 10^{3} \pm 0.09 \cdot 10^{3} \mathrm{M}^{-1}$.

Table S25. Experimental values obtained from ITC titrations of AAC $(P)_{4}-\mathbf{1}$ with 2,2,3-trimethylbutan-1-ol 9 in $n$-octane calculated for $293 \mathrm{~K}$.

\begin{tabular}{cccc}
\hline $\begin{array}{c}K_{\mathrm{a}} \\
\mathrm{M}^{-1}\end{array}$ & $\begin{array}{c}\Delta G_{293 \mathrm{~K}} \\
{\left[\mathrm{kcal} \mathrm{mol}^{-1}\right]}\end{array}$ & $\begin{array}{c}\Delta H \\
{\left[\mathrm{kcal} \mathrm{mol}^{-1}\right]}\end{array}$ & $\begin{array}{c}-\mathrm{T} \Delta S_{293 \mathrm{~K}} \\
{\left[\mathrm{kcal} \mathrm{mol}^{-1}\right]}\end{array}$ \\
\hline $8.2 \cdot 10^{3}$ & -5.3 & -6.0 & +0.7 \\
\hline
\end{tabular}


$( \pm)-\left(2 S^{*}, 3 R^{*}\right)-2,3-$ Dichlorobutan-1-ol $\left(R^{*}, S^{*}\right)-7$

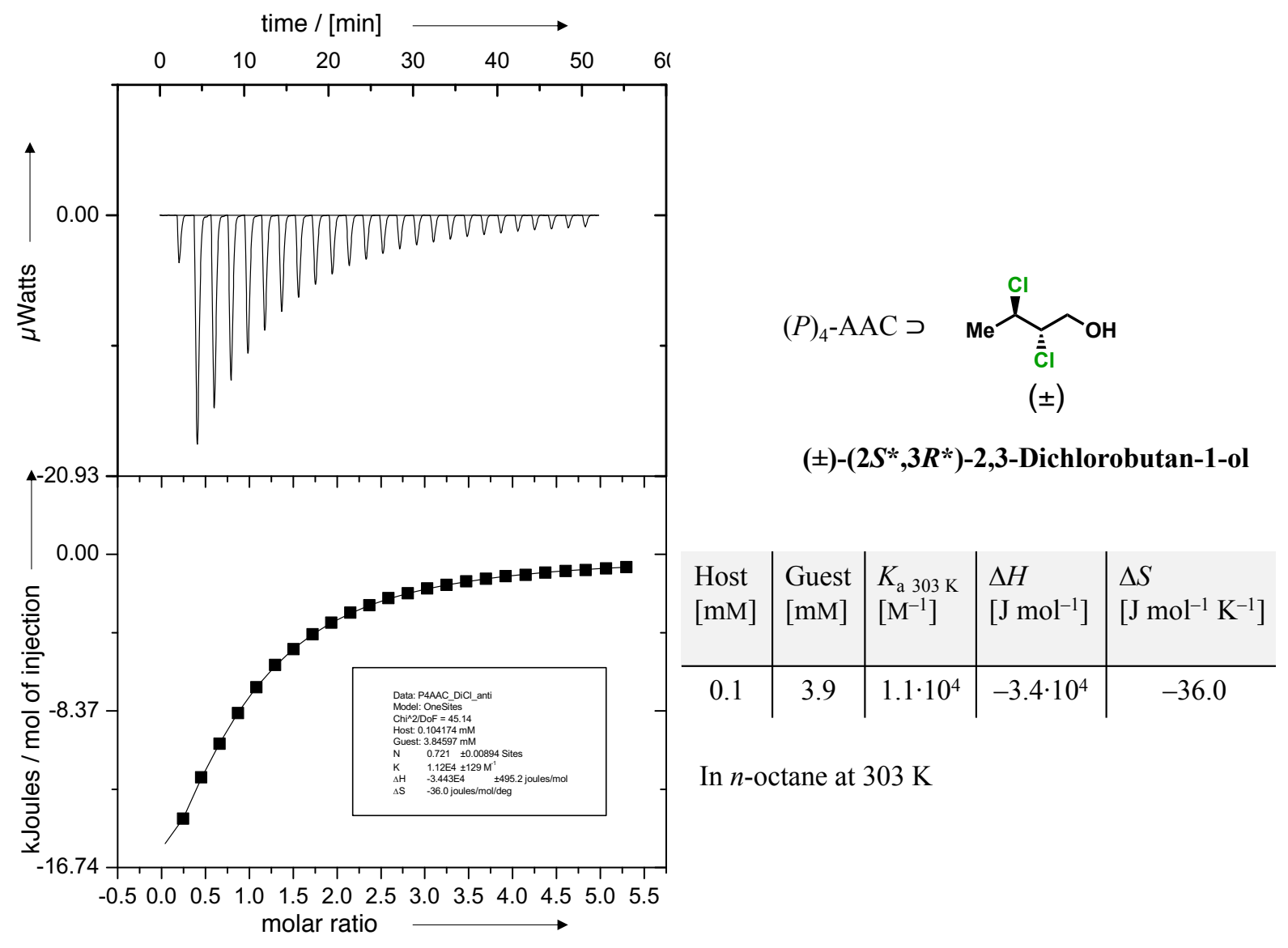

Figure S26. ITC binding isotherm of AAC $(P)_{4}-1$ with $( \pm)-\left(2 S^{*}, 3 R^{*}\right)$-2,3-dichlorobutan-1-ol $\left(R^{*}, S^{*}\right)-7$ in $n$ octane at $303 \mathrm{~K} . K_{\mathrm{a}}=1.12 \cdot 10^{4} \pm 0.01 \cdot 10^{4} \mathrm{M}^{-1}$.

Table S26. Experimental values obtained from ITC titrations of AAC $(P)_{4}-\mathbf{1}$ with $( \pm)-\left(2 S^{*}, 3 R^{*}\right)-2,3-$ dichlorobutan-1-ol $\left(R^{*}, S^{*}\right)-7$ in $n$-octane calculated for $293 \mathrm{~K}$.

\begin{tabular}{cccc}
\hline $\begin{array}{c}K_{\mathrm{a}} \\
\mathrm{M}^{-1}\end{array}$ & $\begin{array}{c}\Delta G_{293 \mathrm{~K}} \\
{\left[\mathrm{kcal} \mathrm{mol}^{-1}\right]}\end{array}$ & $\begin{array}{c}\Delta H \\
{\left[\mathrm{kcal} \mathrm{mol}^{-1}\right]}\end{array}$ & $\begin{array}{c}-\mathrm{T} \Delta S_{293 \mathrm{~K}} \\
{\left[\mathrm{kcal} \mathrm{mol}^{-1}\right]}\end{array}$ \\
\hline $1.8 \cdot 10^{4}$ & -5.7 & -8.2 & +2.5 \\
\hline
\end{tabular}


$( \pm)-\left(2 R^{*}, 3 R^{*}\right)-2,3-D i c h l o r o b u t a n-1-o l ~\left(R^{*}, R^{*}\right)-7$

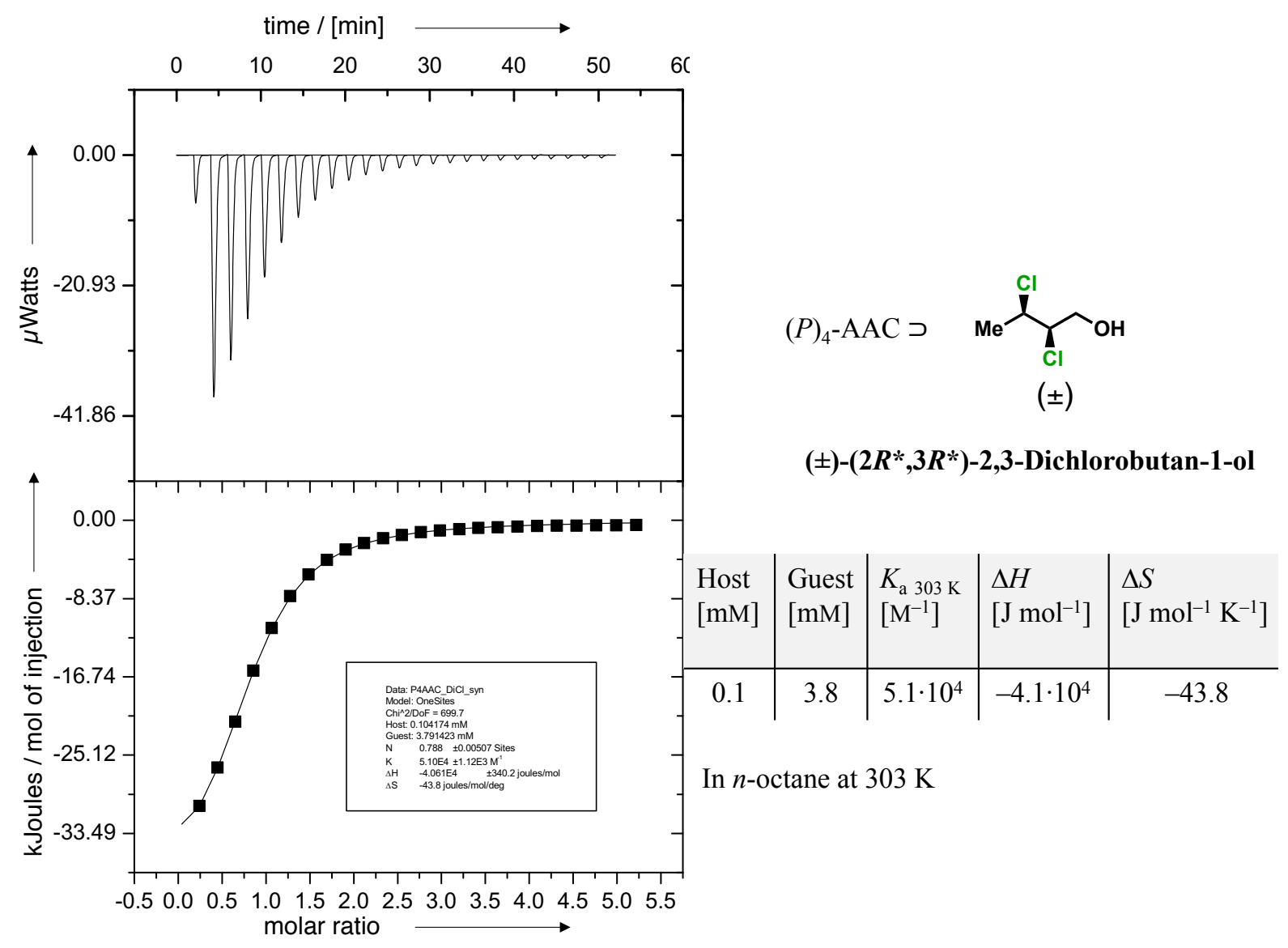

Figure S27. ITC binding isotherm of AAC $(P)_{4}-1$ with $( \pm)-\left(2 R^{*}, 3 R^{*}\right)$-2,3-dichlorobutan-1-ol $\left(R^{*}, R^{*}\right)$-7 in $n$ octane at $303 \mathrm{~K} . K_{\mathrm{a}}=5.10 \cdot 10^{4} \pm 0.11 \cdot 10^{4} \mathrm{M}^{-1}$.

Table S27. Experimental values obtained from ITC titrations of AAC $(P)_{4}-1$ with $( \pm)-\left(2 R^{*}, 3 R^{*}\right)-2,3-$ dichlorobutan-1-ol $\left(R^{*}, R^{*}\right)-7$ in $n$-octane calculated for $293 \mathrm{~K}$.

\begin{tabular}{cccc}
\hline $\begin{array}{c}K_{\mathrm{a}} \\
\mathrm{M}^{-1}\end{array}$ & $\begin{array}{c}\Delta G_{293 \mathrm{~K}} \\
{\left[\mathrm{kcal} \mathrm{mol}^{-1}\right]}\end{array}$ & $\begin{array}{c}\Delta H \\
{\left[\mathrm{kcal} \mathrm{mol}^{-1}\right]}\end{array}$ & $\begin{array}{c}-\mathrm{T} \Delta S_{293 \mathrm{~K}} \\
{\left[\mathrm{kcal} \mathrm{mol}^{-1}\right]}\end{array}$ \\
\hline $9.0 \cdot 10^{4}$ & -6.6 & -9.7 & +3.1 \\
\hline
\end{tabular}


$( \pm)-\left(2 S^{*}, 3 R^{*}\right)-2,3-D i b r o m o b u t a n-1-o l ~\left(R^{*}, S^{*}\right)-8$

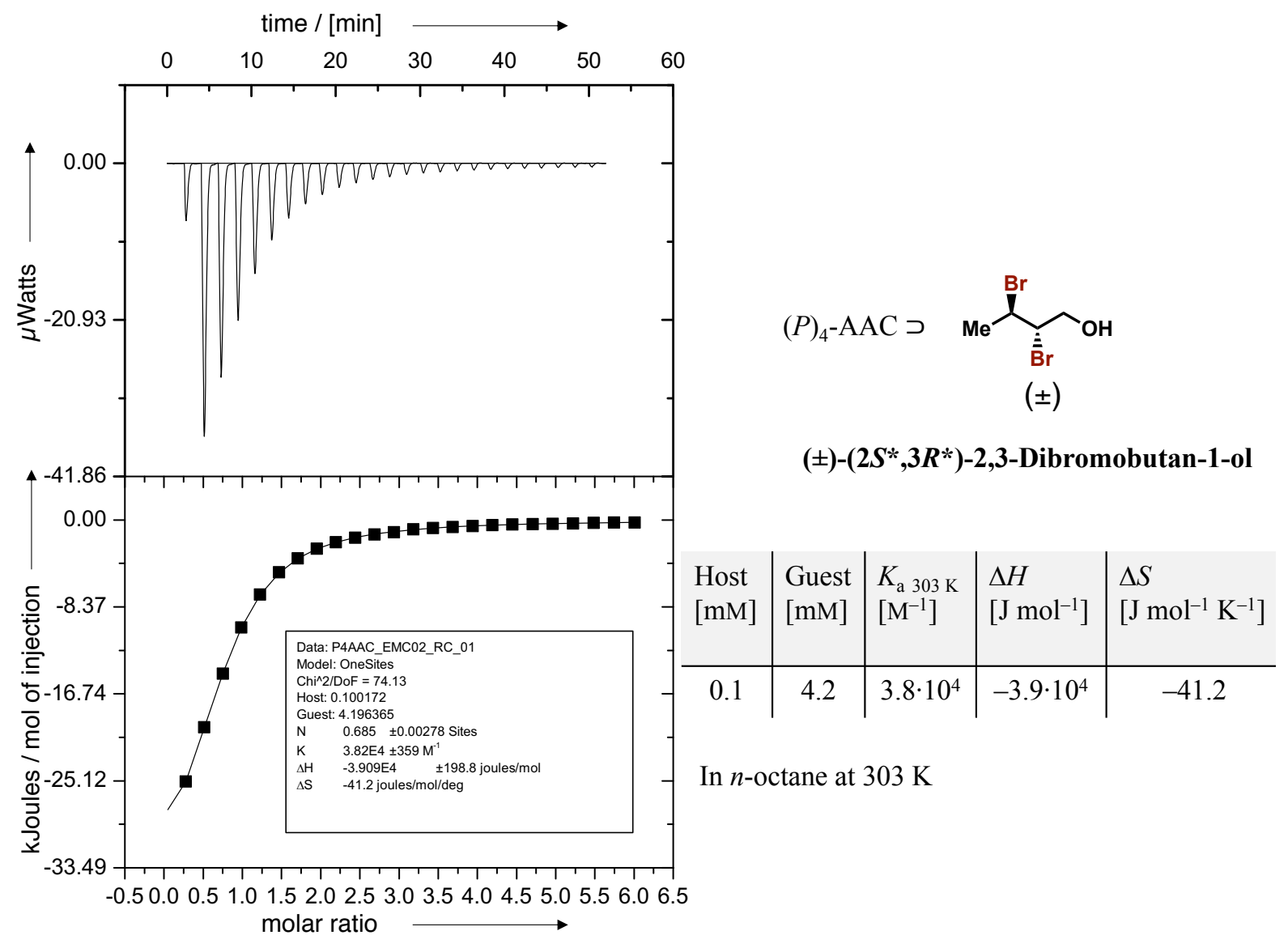

Figure S28. ITC binding isotherm of AAC $(P)_{4}-\mathbf{1}$ with $( \pm)-\left(2 S^{*}, 3 R^{*}\right)$-2,3-dibromobutan-1-ol $\left(R^{*}, S^{*}\right)-\mathbf{8}$ in $n$ octane at $303 \mathrm{~K} . K_{\mathrm{a}}=3.82 \cdot 10^{4} \pm 0.04 \cdot 10^{4} \mathrm{M}^{-1}$.

Table S28. Experimental values obtained from ITC titrations of AAC $(P)_{4}-1$ with $( \pm)-\left(2 S^{*}, 3 R^{*}\right)-2,3-$ dibromobutan-1-ol $\left(R^{*}, S^{*}\right)-8$ in $n$-octane calculated for $293 \mathrm{~K}$.

\begin{tabular}{cccc}
\hline $\begin{array}{c}K_{\mathrm{a}} \\
\mathrm{M}^{-1}\end{array}$ & $\begin{array}{c}\Delta G_{293 \mathrm{~K}} \\
{\left[\mathrm{kcal} \mathrm{mol}^{-1}\right]}\end{array}$ & $\begin{array}{c}\Delta H \\
{\left[\mathrm{kcal} \mathrm{mol}^{-1}\right]}\end{array}$ & $\begin{array}{c}-\mathrm{T} \Delta S_{293 \mathrm{~K}} \\
{\left[\mathrm{kcal} \mathrm{mol}^{-1}\right]}\end{array}$ \\
\hline $6.6 \cdot 10^{4}$ & -6.5 & -9.4 & +2.9 \\
\hline
\end{tabular}


(-)-(2R,3S)-2,3-Dibromobutan-1-ol $(R, S)-8$

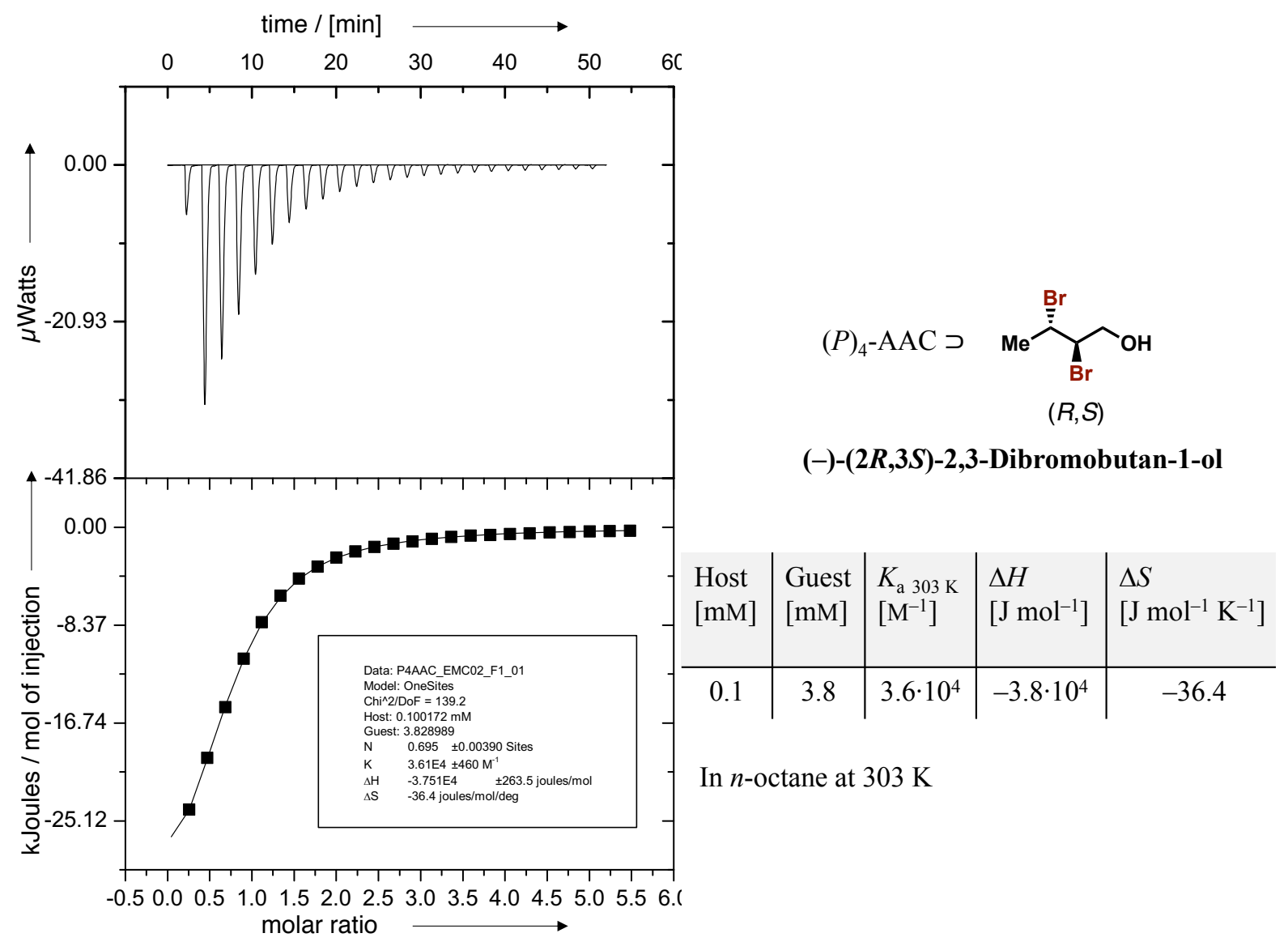

Figure S29. ITC binding isotherm of AAC $(P)_{4}$-1 with (-)-(2R,3S)-2,3-dibromobutan-1-ol $(R, S)$-8 in $n$-octane at $303 \mathrm{~K} . K_{\mathrm{a}}=3.61 \cdot 10^{4} \pm 0.05 \cdot 10^{4} \mathrm{M}^{-1}$.

Table S29. Experimental values obtained from ITC titrations of AAC $(P)_{4}-\mathbf{1}$ with $(-)-(2 R, 3 S)-2,3$-dibromobutan1 -ol $(R, S)-8$ in $n$-octane calculated for $293 \mathrm{~K}$.

\begin{tabular}{cccc}
\hline $\begin{array}{c}K_{\mathrm{a}} \\
\mathrm{M}^{-1}\end{array}$ & $\begin{array}{c}\Delta G_{293 \mathrm{~K}} \\
{\left[\mathrm{kcal} \mathrm{mol}^{-1}\right]}\end{array}$ & $\begin{array}{c}\Delta H \\
{\left[\mathrm{kcal} \mathrm{mol}^{-1}\right]}\end{array}$ & $\begin{array}{c}-\mathrm{T} \Delta S_{293 \mathrm{~K}} \\
{\left[\mathrm{kcal} \mathrm{mol}^{-1}\right]}\end{array}$ \\
\hline $6.1 \cdot 10^{4}$ & -6.4 & -8.9 & +2.5 \\
\hline
\end{tabular}


(+)-(2S,3R)-2,3-Dibromobutan-1-ol $(S, R)-8$

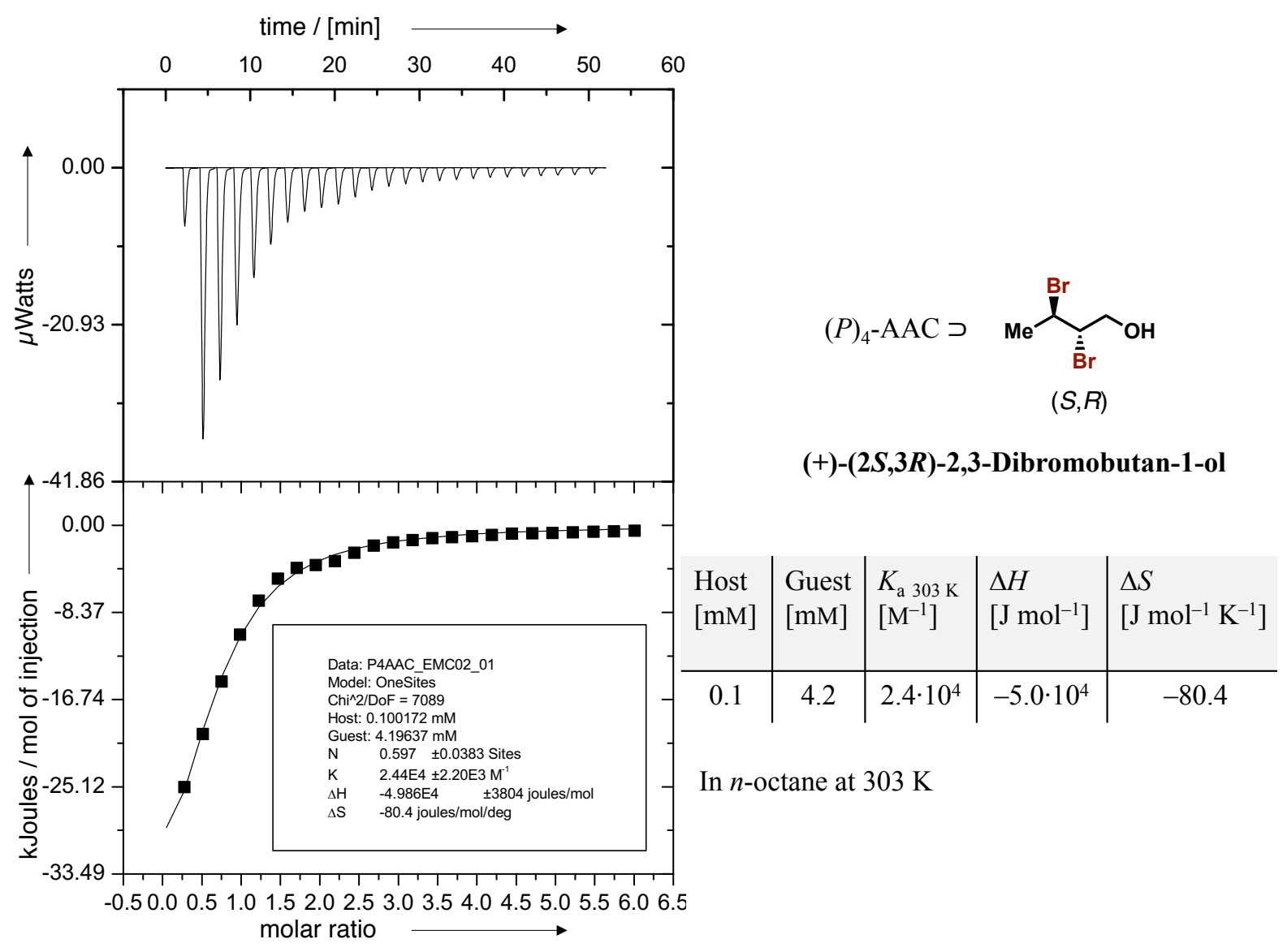

Figure S30. ITC binding isotherm of AAC $(P)_{4}-\mathbf{1}$ with $(+)-(2 S, 3 R)$-2,3-dibromobutan-1-ol $(S, R)-\mathbf{8}$ in $n$-octane at $303 \mathrm{~K} . K_{\mathrm{a}}=2.44 \cdot 10^{4} \pm 0.22 \cdot 10^{4} \mathrm{M}^{-1}$.

Table S30. Experimental values obtained from ITC titrations of AAC $(P)_{4}-\mathbf{1}$ with $(+)-(2 S, 3 R)-2,3$-dibromobutan1 -ol $(S, R)-8$ in $n$-octane calculated for $293 \mathrm{~K}$.

\begin{tabular}{cccc}
\hline$K_{\mathrm{a}}$ & $\Delta G_{293 \mathrm{~K}}$ & $\Delta H$ & $-\mathrm{T} \Delta S_{293 \mathrm{~K}}$ \\
$\mathrm{M}^{-1}$ & {$\left[\mathrm{kcal} \mathrm{mol}^{-1}\right]$} & {$\left[\mathrm{kcal} \mathrm{mol}^{-1}\right]$} & {$\left[\mathrm{kcal} \mathrm{mol}^{-1}\right]$} \\
\hline $4.9 \cdot 10^{4}$ & -6.3 & -11.9 & +5.6 \\
\hline
\end{tabular}


$( \pm)-\left(2 R^{*}, 3 R^{*}\right)-2,3-D i b r o m o b u t a n-1-o l ~\left(R^{*}, R^{*}\right)-8$

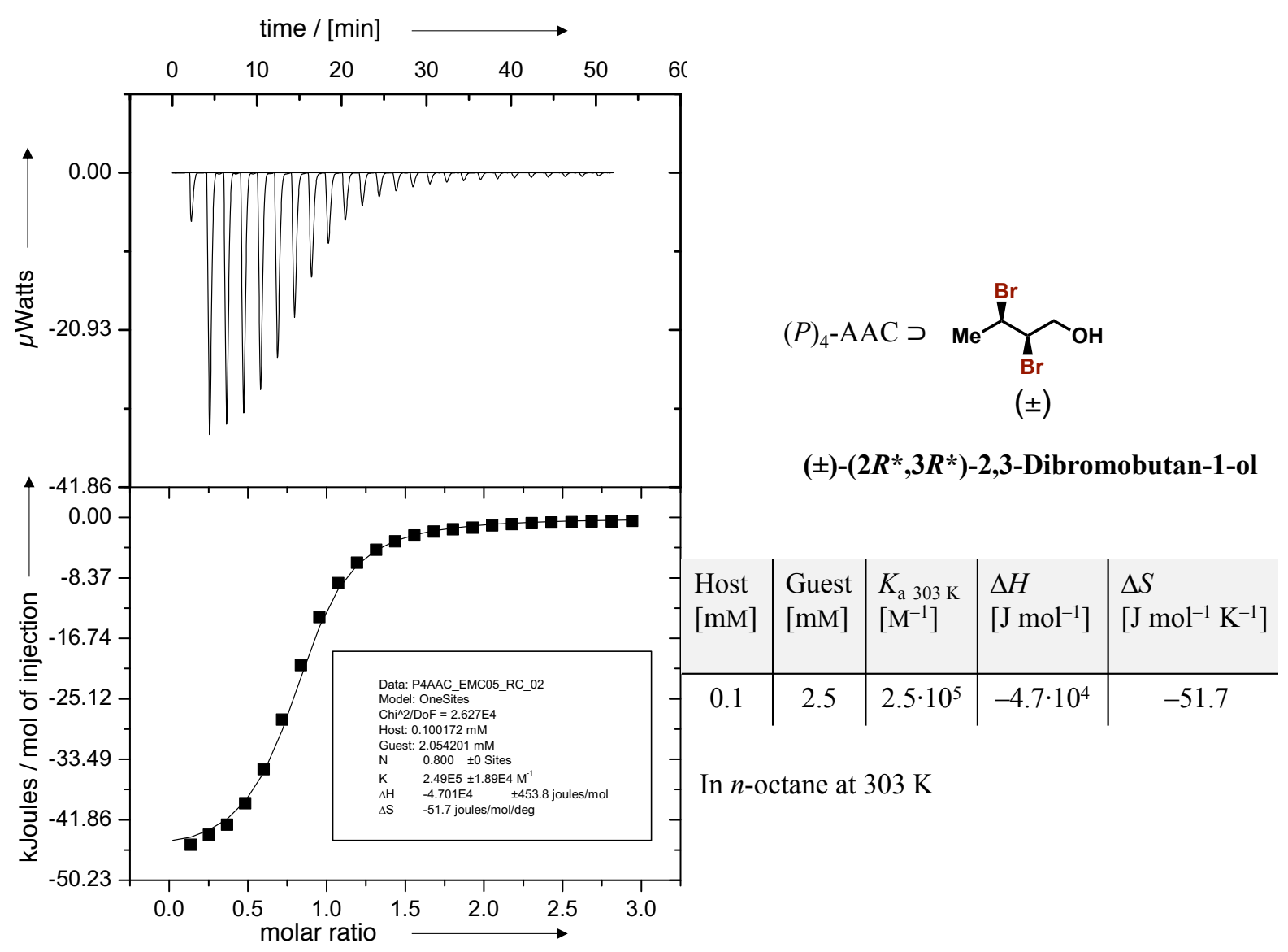

Figure S31. ITC binding isotherm of AAC $(P)_{4}-1$ with $( \pm)-\left(2 R^{*}, 3 R^{*}\right)-2,3$-dibromobutan-1-ol $\left(R^{*}, R^{*}\right)-8$ in $n$ octane at $303 \mathrm{~K} . K_{\mathrm{a}}=2.49 \cdot 10^{5} \pm 0.19 \cdot 10^{5} \mathrm{M}^{-1}$.

Table S31. Experimental values obtained from ITC titrations of AAC $(P)_{4}-1$ with $( \pm)-\left(2 R^{*}, 3 R^{*}\right)-2,3-$ dibromobutan-1-ol $\left(R^{*}, R^{*}\right)-8$ in $n$-octane calculated for $293 \mathrm{~K}$.

\begin{tabular}{cccc}
\hline $\begin{array}{c}K_{\mathrm{a}} \\
\mathrm{M}^{-1}\end{array}$ & $\begin{array}{c}\Delta G_{293 \mathrm{~K}} \\
{\left[\mathrm{kcal} \mathrm{mol}^{-1}\right]}\end{array}$ & $\begin{array}{c}\Delta H \\
{\left[\mathrm{kcal} \mathrm{mol}^{-1}\right]}\end{array}$ & $\begin{array}{c}-\mathrm{T} \Delta S_{293 \mathrm{~K}} \\
{\left[\mathrm{kcal} \mathrm{mol}^{-1}\right]}\end{array}$ \\
\hline $4.8 \cdot 10^{4}$ & -7.6 & -11.2 & +3.6 \\
\hline
\end{tabular}


(+)-(2R,3R)-2,3-Dibromobutan-1-ol $(R, R)-8$

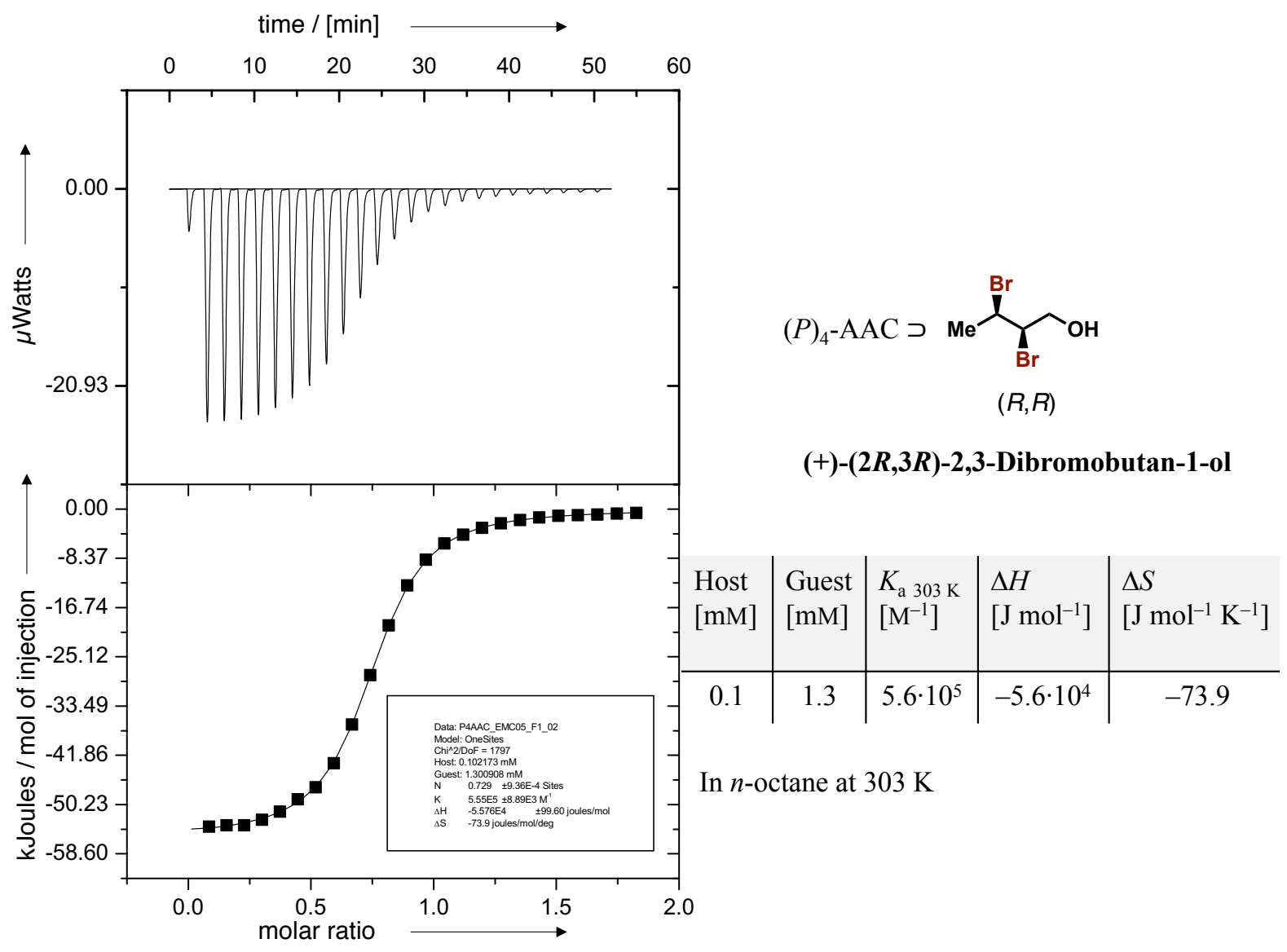

Figure S32. ITC binding isotherm of AAC $(P)_{4}-\mathbf{1}$ with $(+)-(2 R, 3 R)$-2,3-dibromobutan-1-ol $(R, R)-8$ in $n$-octane at $303 \mathrm{~K} . K_{\mathrm{a}}=5.55 \cdot 10^{5} \pm 0.09 \cdot 10^{5} \mathrm{M}^{-1}$.

Table S32. Experimental values obtained from ITC titrations of AAC $(P)_{4}-1$ with $(+)-(2 R, 3 R)$-2,3-dibromobutan1 -ol $(R, R)-8$ in $n$-octane calculated for $293 \mathrm{~K}$.

\begin{tabular}{cccc}
\hline $\begin{array}{c}K_{\mathrm{a}} \\
\mathrm{M}^{-1}\end{array}$ & $\begin{array}{c}\Delta G_{293 \mathrm{~K}} \\
{\left[\mathrm{kcal} \mathrm{mol}^{-1}\right]}\end{array}$ & $\begin{array}{c}\Delta H \\
{\left[\mathrm{kcal} \mathrm{mol}^{-1}\right]}\end{array}$ & $\begin{array}{c}-\mathrm{T} \Delta S_{293 \mathrm{~K}} \\
{\left[\mathrm{kcal} \mathrm{mol}^{-1}\right]}\end{array}$ \\
\hline $1.2 \cdot 10^{6}$ & -8.1 & -13.3 & +5.2 \\
\hline
\end{tabular}




\section{(-)-(2S,3S)-2,3-Dibromobutan-1-ol $(S, S)-8$}

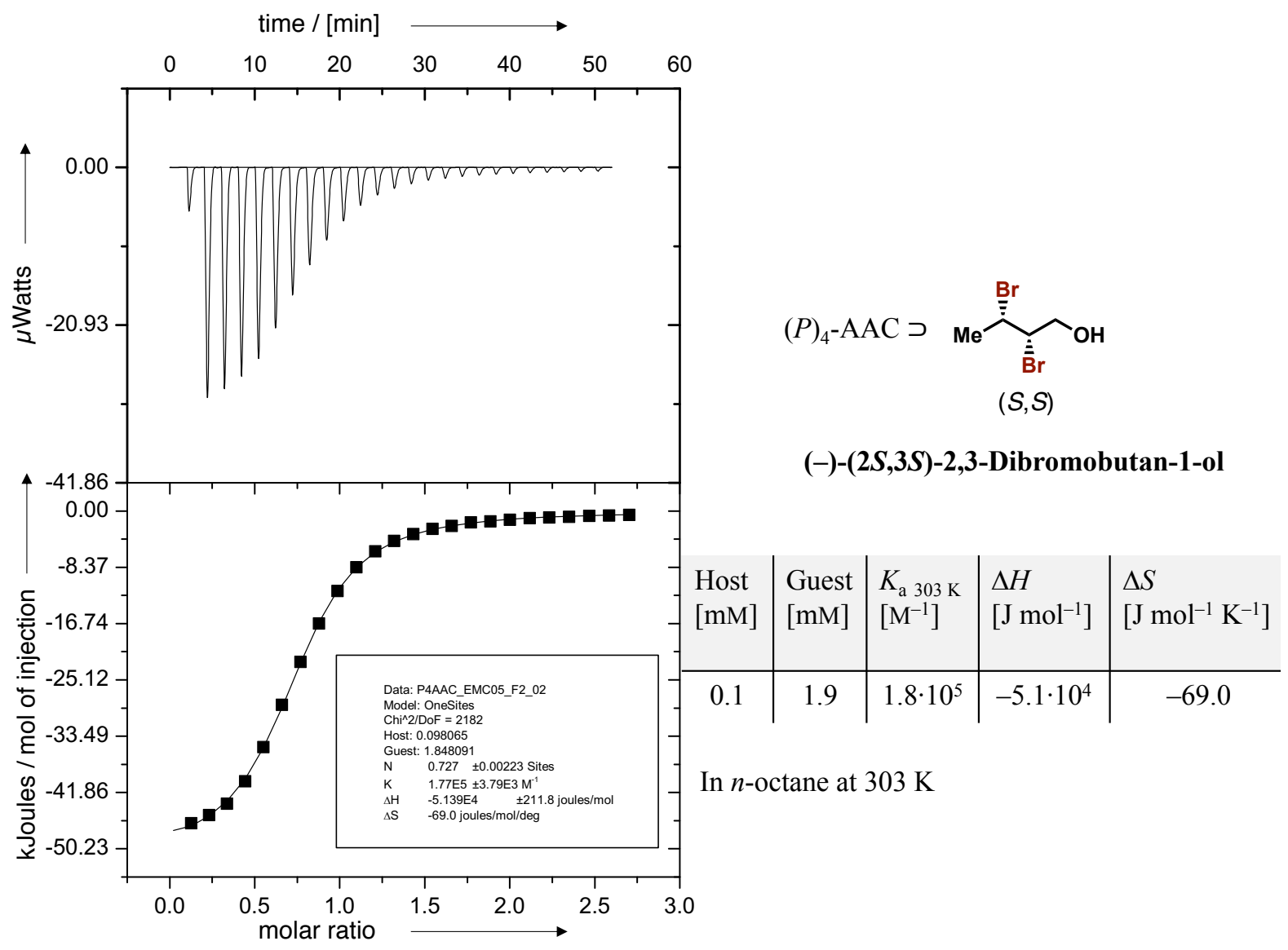

Figure S33. ITC binding isotherm of AAC $(P)_{4}-\mathbf{1}$ with (-)-(2S,3S)-2,3-dibromobutan-1-ol $(S, S)-8$ in $n$-octane at 303 K. $K_{\mathrm{a}}=1.77 \cdot 10^{5} \pm 0.04 \cdot 10^{5} \mathrm{M}^{-1}$.

Table S33. Experimental values obtained from ITC titrations of AAC $(P)_{4}-1$ with (-)-(2S,3S)-2,3-dibromobutan1 -ol $(S, S)$-8 in $n$-octane calculated for $293 \mathrm{~K}$.

\begin{tabular}{cccc}
\hline $\begin{array}{c}K_{\mathrm{a}} \\
\mathrm{M}^{-1}\end{array}$ & $\begin{array}{c}\Delta G_{293 \mathrm{~K}} \\
{\left[\mathrm{kcal} \mathrm{mol}^{-1}\right]}\end{array}$ & $\begin{array}{c}\Delta H \\
{\left[\mathrm{kcal} \mathrm{mol}^{-1}\right]}\end{array}$ & $\begin{array}{c}-\mathrm{T} \Delta S_{293 \mathrm{~K}} \\
{\left[\mathrm{kcal} \mathrm{mol}^{-1}\right]}\end{array}$ \\
\hline $3.6 \cdot 10^{5}$ & -7.5 & -12.3 & +4.8 \\
\hline
\end{tabular}


$( \pm)-\left(2 S^{*}, 3 R^{*}\right)-2,3-D i b r o m o-2-m e t h y l b u t a n-1-o l ~\left(R^{*}, S^{*}\right)-10$

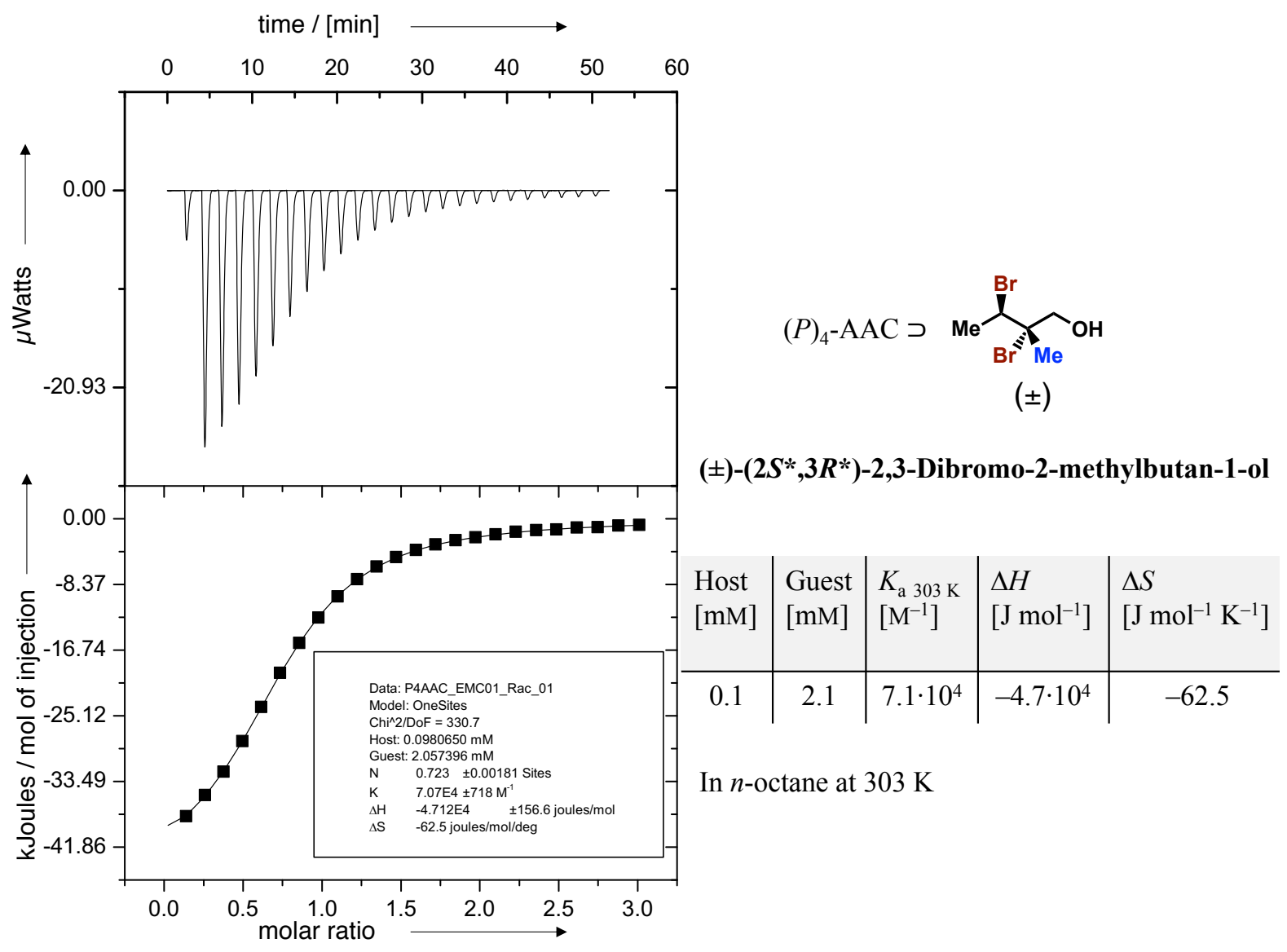

Figure S34. ITC binding isotherm of AAC $(P)_{4}-1 \mathrm{C}$ with $( \pm)-\left(2 S^{*}, 3 R^{*}\right)$-2,3-dibromo-2-methylbutan-1-ol $\left(R^{*}, S^{*}\right)$ 10 in $n$-octane at $303 \mathrm{~K} . K_{\mathrm{a}}=7.1 \cdot 10^{4} \pm 0.07 \cdot 10^{4} \mathrm{M}^{-1}$.

Table S34. Experimental values obtained from ITC titrations of AAC $(P)_{4}-1$ with $( \pm)-\left(2 S^{*}, 3 R^{*}\right)-2,3$-dibromo-2methylbutan-1-ol $\left(R^{*}, S^{*}\right)-\mathbf{1 0}$ in $n$-octane calculated for $293 \mathrm{~K}$.

\begin{tabular}{cccc}
\hline $\begin{array}{c}K_{\mathrm{a}} \\
\mathrm{M}^{-1}\end{array}$ & $\begin{array}{c}\Delta G_{293 \mathrm{~K}} \\
{\left[\mathrm{kcal} \mathrm{mol}^{-1}\right]}\end{array}$ & $\begin{array}{c}\Delta H \\
{\left[\mathrm{kcal} \mathrm{mol}^{-1}\right]}\end{array}$ & $\begin{array}{c}-\mathrm{T} \Delta S_{293 \mathrm{~K}} \\
{\left[\mathrm{kcal} \mathrm{mol}^{-1}\right]}\end{array}$ \\
\hline $1.4 \cdot 10^{5}$ & -6.9 & -11.1 & +4.2 \\
\hline
\end{tabular}


$( \pm)-\left(2 R^{*}, 3 R^{*}\right)-2,3-D i b r o m o-2-m e t h y l b u t a n-1-o l ~\left(R^{*}, R^{*}\right)-10$

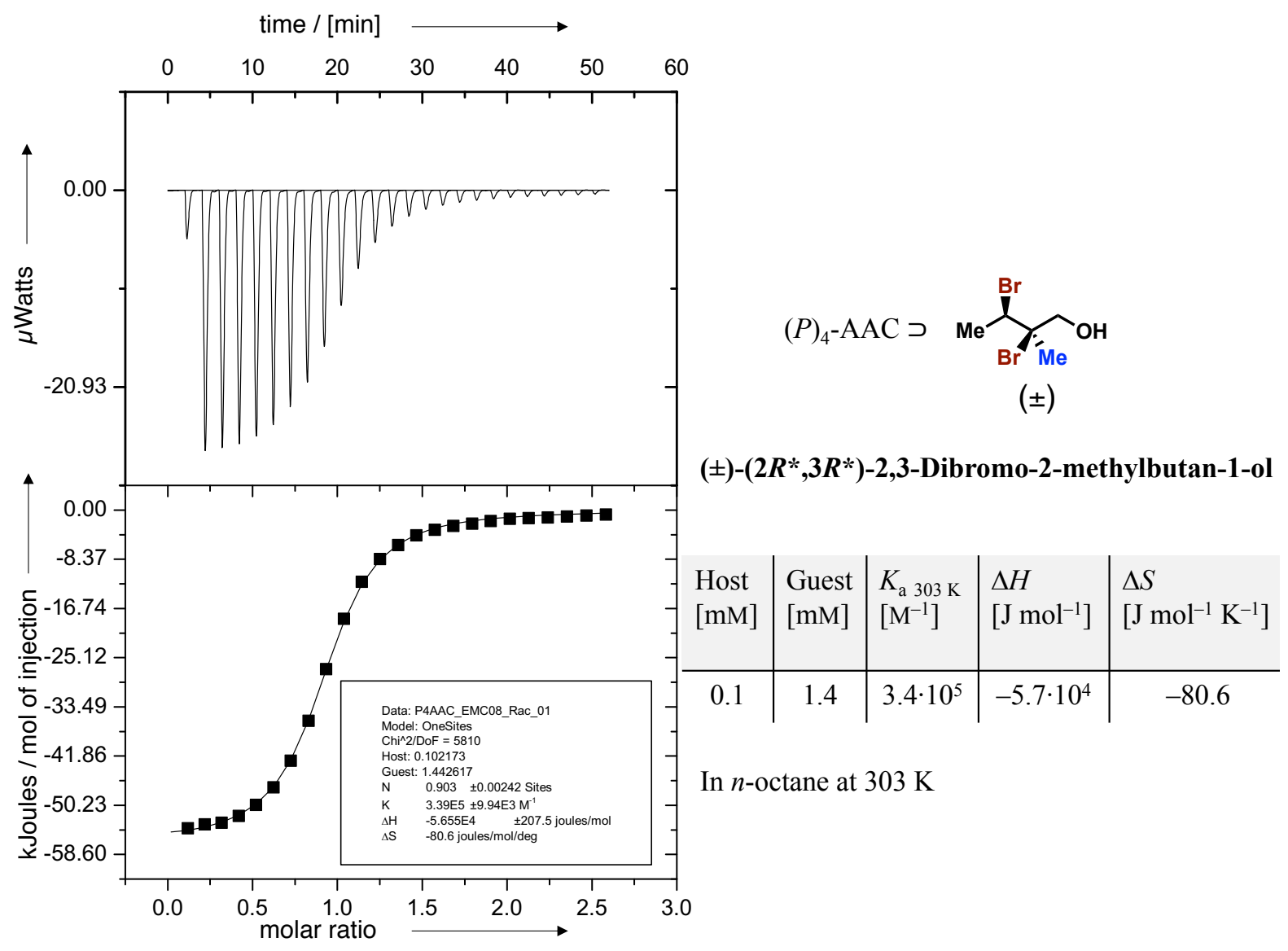

Figure S35. ITC binding isotherm of AAC $(P)_{4}$-1 with $( \pm)-\left(2 R^{*}, 3 R^{*}\right)$-2,3-dibromo-2-methylbutan-1-ol $\left(R^{*}, R^{*}\right)$ 10 in $n$-octane at $303 \mathrm{~K} . K_{\mathrm{a}}=3.39 \cdot 10^{5} \pm 0.10 \cdot 10^{5} \mathrm{M}^{-1}$.

Table S35. Experimental values obtained from ITC titrations of AAC $(P)_{4}-1$ with $( \pm)-\left(2 R^{*}, 3 R^{*}\right)-2,3$-dibromo-2methylbutan-1-ol $\left(R^{*}, R^{*}\right)$-10 in $n$-octane calculated for $293 \mathrm{~K}$.

\begin{tabular}{cccc}
\hline $\begin{array}{c}K_{\mathrm{a}} \\
\mathrm{M}^{-1}\end{array}$ & $\begin{array}{c}\Delta G_{293 \mathrm{~K}} \\
{\left[\mathrm{kcal} \mathrm{mol}^{-1}\right]}\end{array}$ & $\begin{array}{c}\Delta H \\
{\left[\mathrm{kcal} \mathrm{mol}^{-1}\right]}\end{array}$ & $\begin{array}{c}-\mathrm{T} \Delta S_{293 \mathrm{~K}} \\
{\left[\mathrm{kcal} \mathrm{mol}^{-1}\right]}\end{array}$ \\
\hline $7.4 \cdot 10^{5}$ & -7.9 & -13.5 & +5.6 \\
\hline
\end{tabular}


(+)-(2R,3R)-2,3-Dibromo-2-methylbutan-1-ol $(R, R)-10$

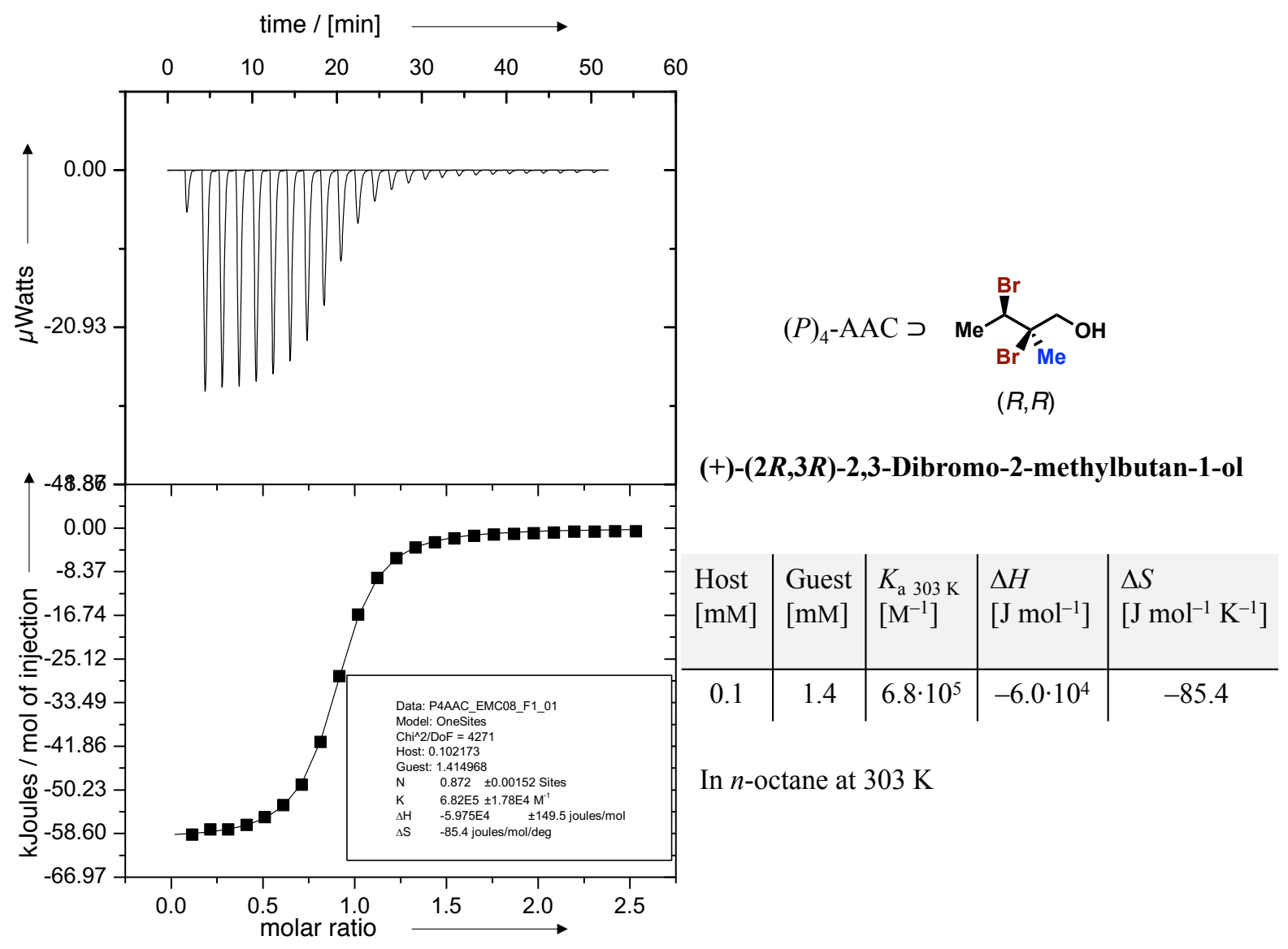

Figure S36. ITC binding isotherm of AAC $(P)_{4}$-1 with $(+)-(2 R, 3 R)$-2,3-dibromo-2-methylbutan-1-ol $(R, R)-\mathbf{1 0}$ in $n$-octane at $303 \mathrm{~K} . K_{\mathrm{a}}=6.82 \cdot 10^{5} \pm 0.18 \cdot 10^{5} \mathrm{M}^{-1}$.

Table S36. Experimental values obtained from ITC titrations of AAC $(P)_{4}-\mathbf{1}$ with (+)-( $\left.2 R, 3 R\right)-2,3$-dibromo-2methylbutan-1-ol $(R, R)-\mathbf{1 0}$ in $n$-octane calculated for $293 \mathrm{~K}$.

\begin{tabular}{cccc}
\hline $\begin{array}{c}K_{\mathrm{a}} \\
\mathrm{M}^{-1}\end{array}$ & $\begin{array}{c}\Delta G_{293 \mathrm{~K}} \\
{\left[\mathrm{kcal} \mathrm{mol}^{-1}\right]}\end{array}$ & $\begin{array}{c}\Delta H \\
{\left[\mathrm{kcal} \mathrm{mol}^{-1}\right]}\end{array}$ & $\begin{array}{c}-\mathrm{T} \Delta S_{293 \mathrm{~K}} \\
{\left[\mathrm{kcal} \mathrm{mol}^{-1}\right]}\end{array}$ \\
\hline $1.6 \cdot 10^{6}$ & -8.3 & -14.3 & +6.0 \\
\hline
\end{tabular}


(-)-(2S,3S)-2,3-Dibromo-2-methylbutan-1-ol $(S, S)-10$

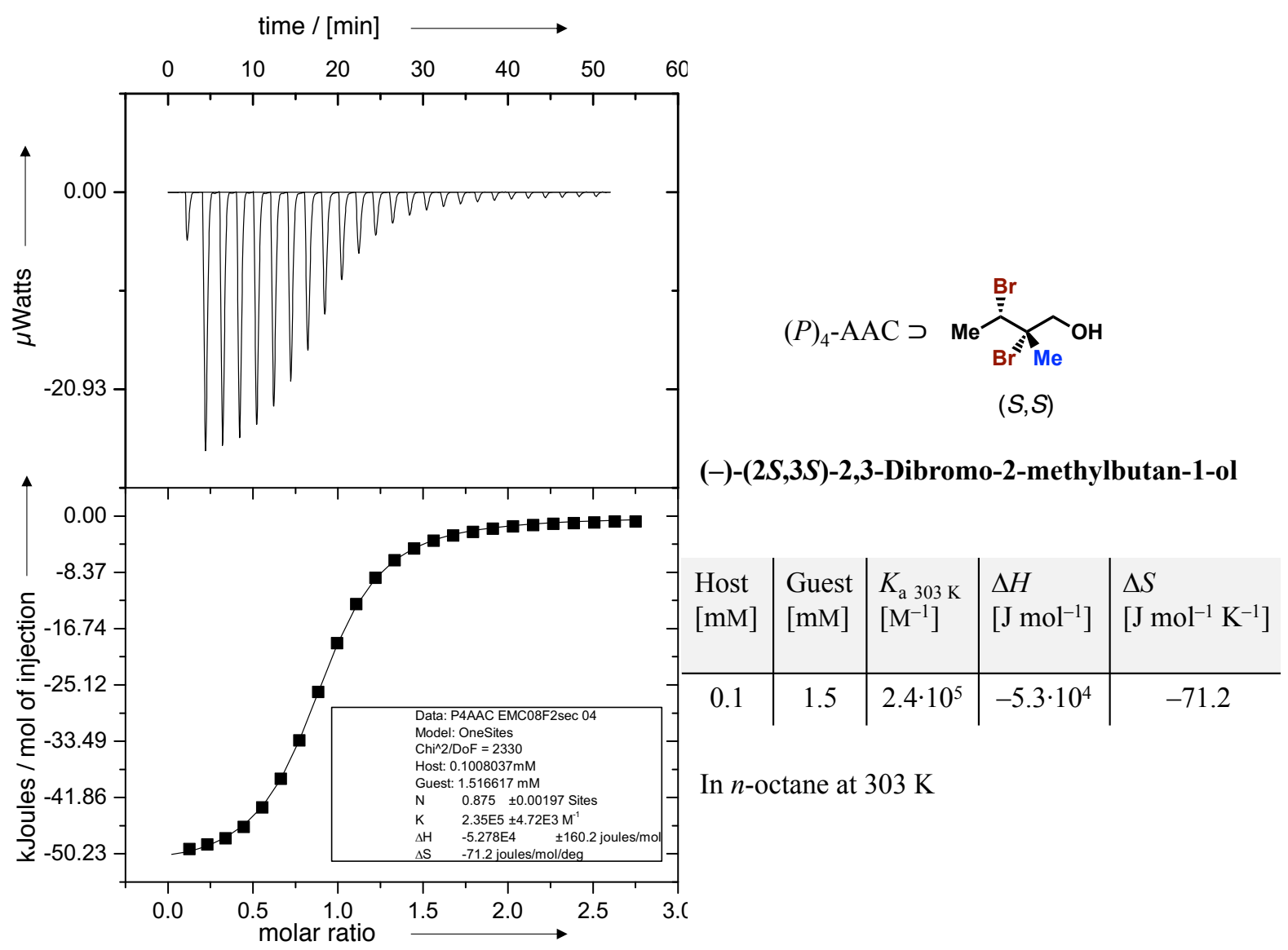

Figure S37. ITC binding isotherm of AAC $(P)_{4}-\mathbf{1}$ with (-)-(2S,3S)-2,3-dibromo-2-methylbutan-1-ol $(S, S)$-10 in $n$-octane at $303 \mathrm{~K} . K_{\mathrm{a}}=2.35 \cdot 10^{5} \pm 0.05 \cdot 10^{5} \mathrm{M}^{-1}$.

Table S37. Experimental values obtained from ITC titrations of AAC $(P)_{4}-\mathbf{1}$ with $(-)-(2 S, 3 S)-2,3$-dibromo-2methylbutan-1-ol $(S, S)$-10 in $n$-octane calculated for $293 \mathrm{~K}$.

\begin{tabular}{cccc}
\hline $\begin{array}{c}K_{\mathrm{a}} \\
\mathrm{M}^{-1}\end{array}$ & $\begin{array}{c}\Delta G_{293 \mathrm{~K}} \\
{\left[\mathrm{kcal} \mathrm{mol}^{-1}\right]}\end{array}$ & $\begin{array}{c}\Delta H \\
{\left[\mathrm{kcal} \mathrm{mol}^{-1}\right]}\end{array}$ & $\begin{array}{c}-\mathrm{T} \Delta S_{293 \mathrm{~K}} \\
{\left[\mathrm{kcal} \mathrm{mol}^{-1}\right]}\end{array}$ \\
\hline $4.9 \cdot 10^{5}$ & -7.6 & -12.6 & +5.0 \\
\hline
\end{tabular}


$( \pm)-\left(2 S^{*}, 3 R^{*}\right)-2,3-D i b r o m o-4,4,4-t r i f l u o r o b u t a n-1-o l ~\left(R^{*}, S^{*}\right)-11$

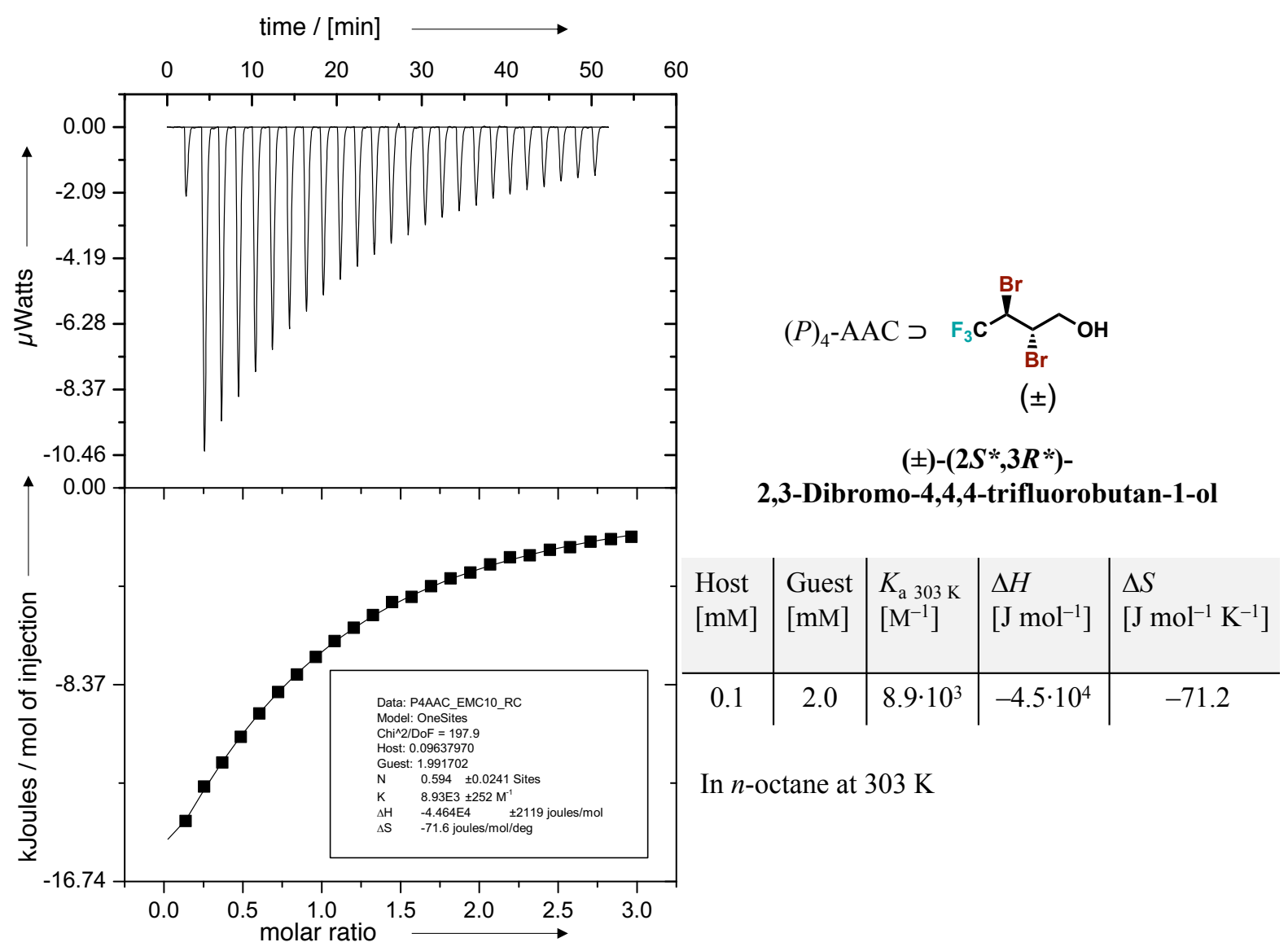

Figure S38. ITC binding isotherm of AAC $(P)_{4}-1$ with $( \pm)-\left(2 S^{*}, 3 R^{*}\right)$-2,3-dibromo-4,4,4-trifluorobutan-1-ol $\left(R^{*}, S^{*}\right)$-11 in $n$-octane at $303 \mathrm{~K} . K_{\mathrm{a}}=8.93 \cdot 10^{3} \pm 0.25 \cdot 10^{3} \mathrm{M}^{-1}$.

Table S38. Experimental values obtained from ITC titrations of AAC $(P)_{4}-1$ with $( \pm)-\left(2 S^{*}, 3 R^{*}\right)-2,3$-dibromo4,4,4-trifluorobutan-1-ol $\left(R^{*}, S^{*}\right)-11$ in $n$-octane calculated for $293 \mathrm{~K}$.

\begin{tabular}{cccc}
\hline$K_{\mathrm{a}}$ & $\Delta G_{293 \mathrm{~K}}$ & $\Delta H$ & $-\mathrm{T} \Delta S_{293 \mathrm{~K}}$ \\
$\mathrm{M}^{-1}$ & {$\left[\mathrm{kcal} \mathrm{mol}^{-1}\right]$} & $\begin{array}{c}\left.\Delta \mathrm{kcal} \mathrm{mol}^{-1}\right] \\
{\left[\mathrm{kcal} \mathrm{mol}^{-1}\right]}\end{array}$ & +5.0 \\
\hline $1.7 \cdot 10^{4}$ & -5.7 & -10.7 & +5 \\
\hline
\end{tabular}




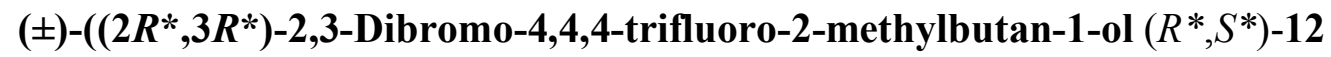

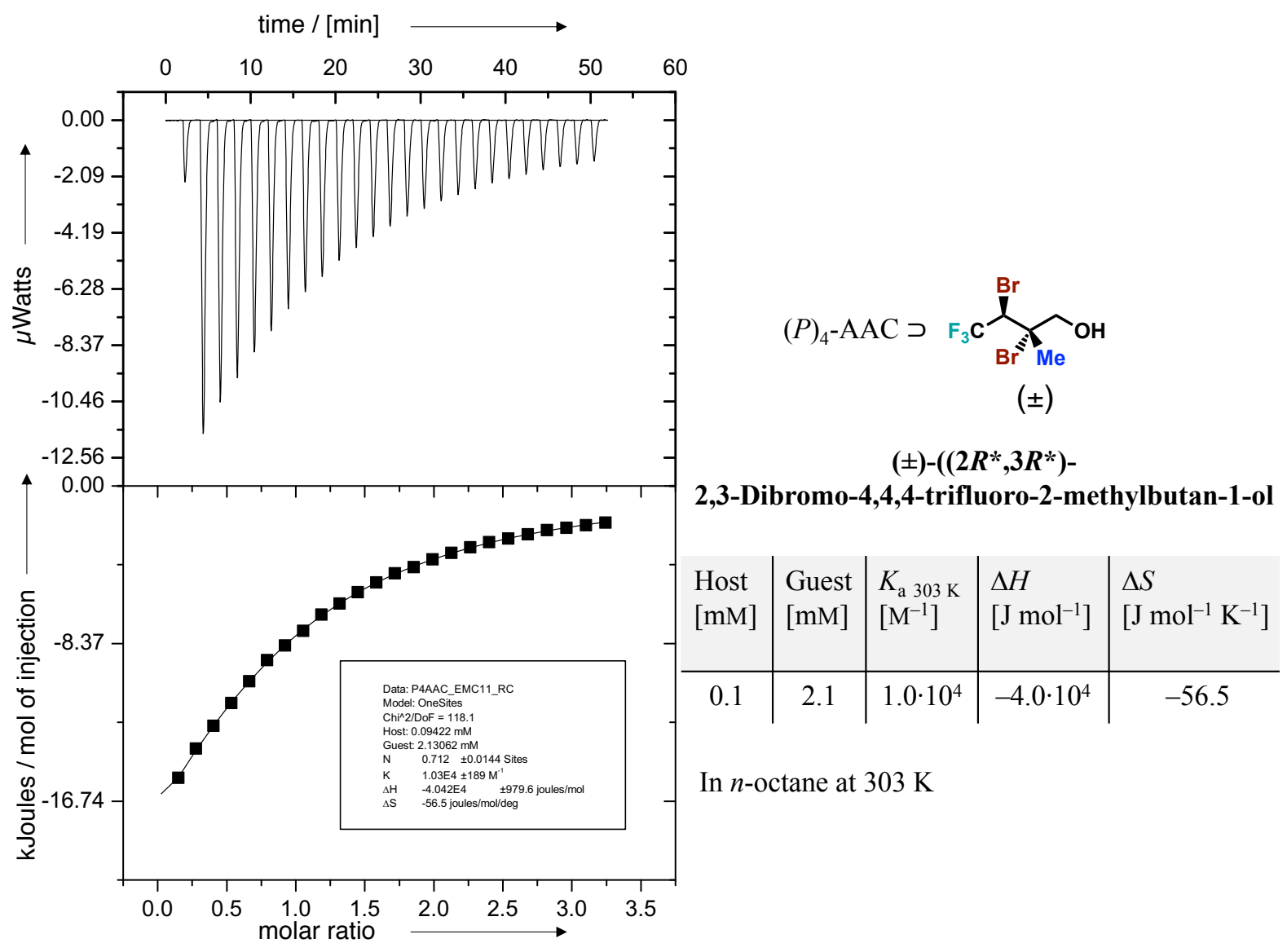

Figure S39. ITC binding isotherm of AAC $(P)_{4}-1$ with $( \pm)-\left(\left(2 R^{*}, 3 R^{*}\right)-2,3\right.$-dibromo-4,4,4-trifluoro-2methylbutan-1-ol $\left(R^{*}, S^{*}\right)-12$ in $n$-octane at $303 \mathrm{~K} . K_{\mathrm{a}}=1.03 \cdot 10^{4} \pm 0.02 \cdot 10^{4} \mathrm{M}^{-1}$.

Table S39. Experimental values obtained from ITC titrations of AAC $(P)_{4}-1$ with $( \pm)-\left(2 R^{*}, 3 R^{*}\right)-2,3$-dibromo4,4,4-trifluoro-2-methylbutan-1-ol $\left(R^{*}, S^{*}\right)$-12 in $n$-octane calculated for $293 \mathrm{~K}$.

\begin{tabular}{cccc}
\hline $\begin{array}{c}K_{\mathrm{a}} \\
\mathrm{M}^{-1}\end{array}$ & $\begin{array}{c}\Delta G_{293 \mathrm{~K}} \\
{\left[\mathrm{kcal} \mathrm{mol}^{-1}\right]}\end{array}$ & $\begin{array}{c}\Delta H \\
{\left[\mathrm{kcal} \mathrm{mol}^{-1}\right]}\end{array}$ & $\begin{array}{c}-\mathrm{T} \Delta S_{293 \mathrm{~K}} \\
{\left[\mathrm{kcal} \mathrm{mol}^{-1}\right]}\end{array}$ \\
\hline $1.8 \cdot 10^{4}$ & -5.7 & -9.7 & +4.0 \\
\hline
\end{tabular}




\section{S6. Summary of Association Constants Obtained from ECD Spectroscopic and ITC Titrations}

Table S40. Summary of association constants $K_{\mathrm{a}}$ as determined by ECD titrations in $n$-octane. $\Delta G_{293} \mathrm{~K}$ was calculated from $K_{\mathrm{a}}$ at $293 \mathrm{~K}$. Errors in $K_{\mathrm{a}}$ are estimated to be in the range of $\pm 20 \%$.

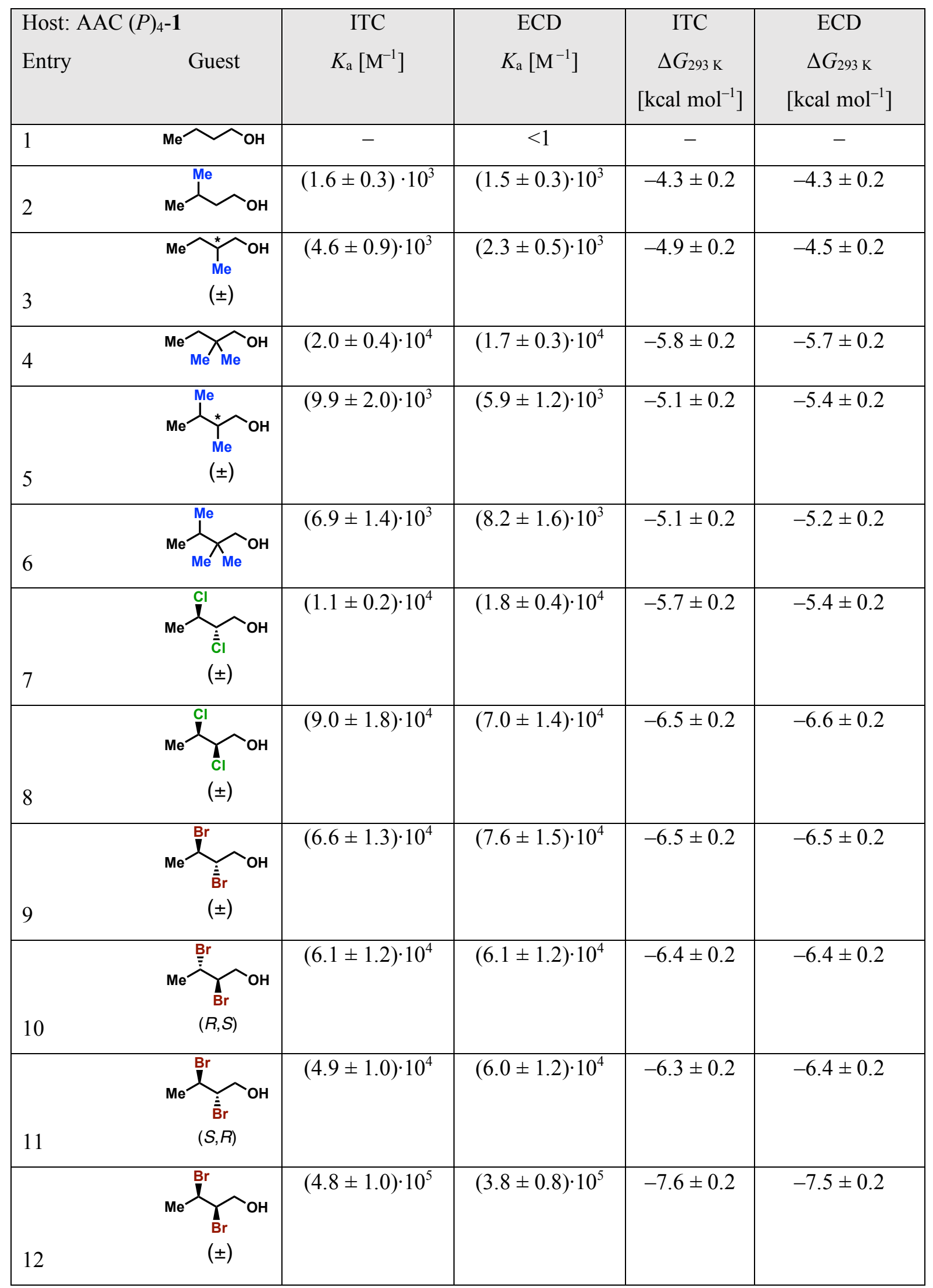




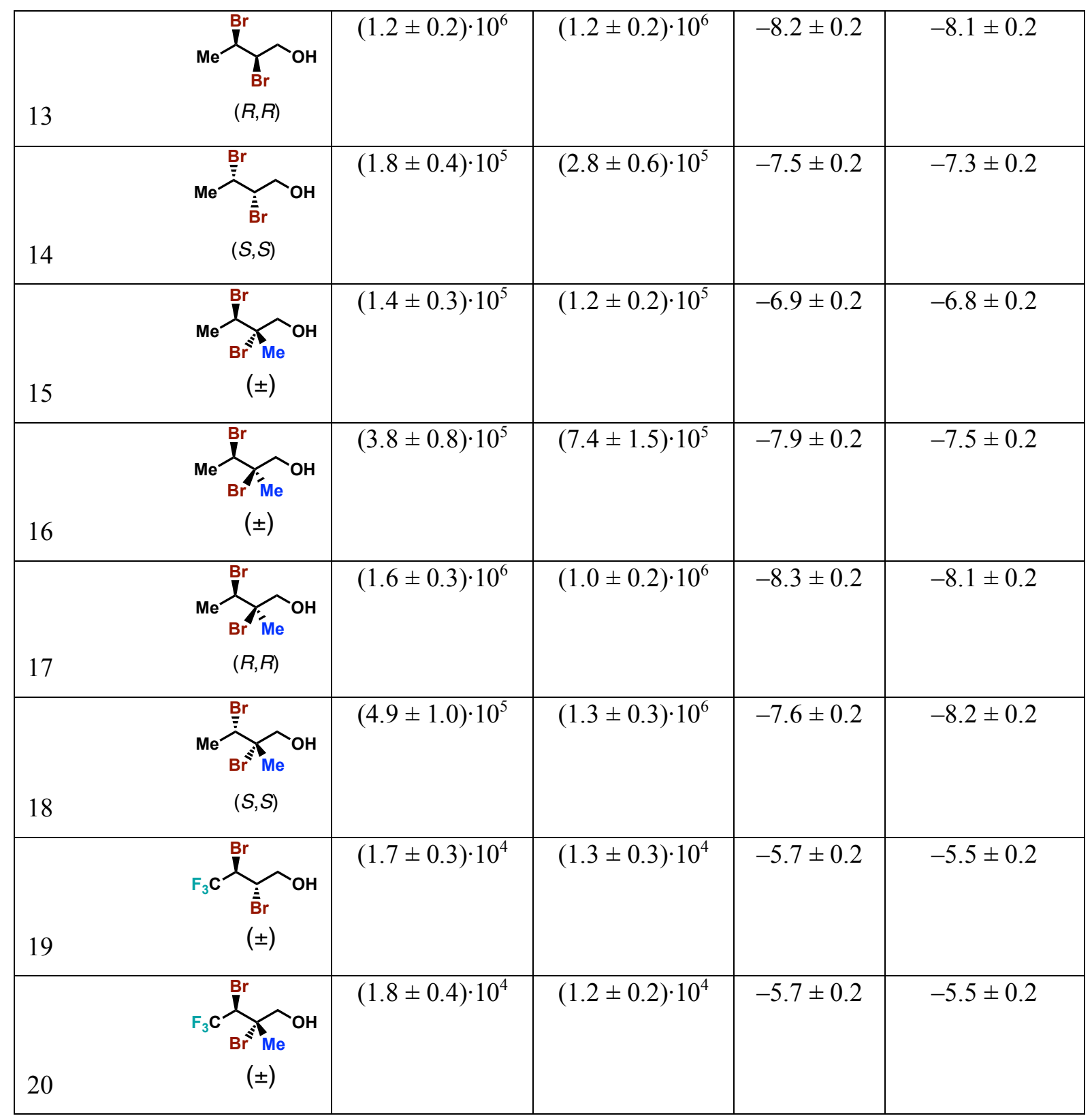




\section{S7. Comparison of the Gibbs Binding Energies for Selected Methyl- and Bromo-}

\section{Containing Alcohols}

Halogen-bonding interactions: $\mathrm{Me} \rightarrow \mathrm{Br}(\mathrm{Br} \cdots \| \mid)$<smiles>CC(C)[C@H](C)O</smiles>

$(R / S)-6$
$-1.1 \mathrm{kcal} \cdot \mathrm{M}^{-1}$<smiles>C[C@@H](Br)[C@H](Br)CO</smiles>

$\left(R^{*}, S^{*}\right)-8$

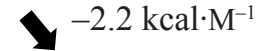<smiles>C[C@@H](Br)[C@H](Br)CO</smiles>

$\left(R^{*}, R^{\star}\right)-8$<smiles>CC(C)C(C)(C)CO</smiles>

9
$-2.1 \mathrm{kcal} \cdot \mathrm{M}^{-1}$<smiles>C[C@H](Br)[C@]([NH3+])(Br)CO</smiles>

$\left(R^{*}, R^{*}\right)-10$

Figure S40. Comparison of the differences in Gibbs binding energies of binding $\Delta \Delta \mathrm{G} 293 \mathrm{~K}$ in $\mathrm{kcal} \mathrm{mol}^{-1} \mathrm{obtained}$ from ECD spectroscopic titrations of the 2,3-dimethylbutanol $(R / \mathrm{S})-\mathbf{6}$ (left) and 2,2,3-trimethylbutanol 9 bound to AAC $(P) 4-\mathbf{1}$ with their anti- and syn-configured bromo analogues $\mathbf{8}$ and 10. ${ }^{[7]}$

\section{S8. NMR Spectra of $(P)_{4}$-Configured AAC with Guest Molecules}

Figure S41 displays the protons which were monitored during guest addition to a solution of AAC $(P)_{4}-1$ in $\left[\mathrm{D}_{18}\right] n$-octane. 1D and 2D NMR spectra of the host-guest complexes were recorded on a Bruker AVIII 600 Ultra Shell spectrometer equipped with a Prodigy Cryo Probe (600 MHz for ${ }^{1} \mathrm{H} ; 150 \mathrm{MHz}$ for ${ }^{13} \mathrm{C}$ ). Low temperature 1D NMR spectroscopic measurements were conducted on a Bruker spectrometer equipped with a Prodigy Cryo Probe (500 MHz for $\left.{ }^{1} \mathrm{H}\right)$.
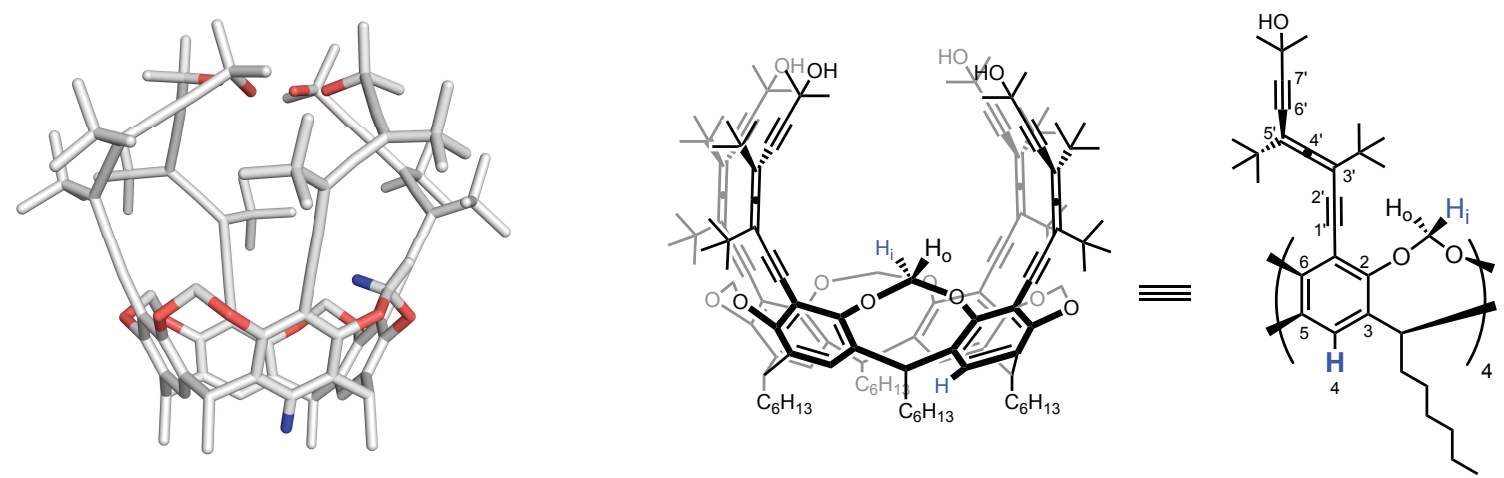

Figure S41. Protons, which were monitored during the binding studies, are highlighted in blue.

Preparation of solutions for NMR spectroscopic experiments (1D and 2D): A solution of the AAC $(P)_{4}-1$ or $(M)_{4} \mathbf{- 1}$, referred to as "host", was prepared gravimetrically $(\sim 10 \mathrm{mM})$ in $\left[\mathrm{D}_{18}\right] n$ octane. A second solution of the "guest" $(\mathbf{2}-\mathbf{1 2})$ was prepared gravimetrically in $\left[\mathrm{D}_{18}\right] n$-octane and subsequently added to the host-solution. Slow exchange of the guests on the NMR timescale allowed for full characterization of the host-guest complexes. In ROESY NMR 
studies, significant correlation of the guests with the inward pointing $\mathrm{H}_{\mathrm{i}}$ of the host was observed. ROESY NMR spectra are shown for selected host-guest complexes.

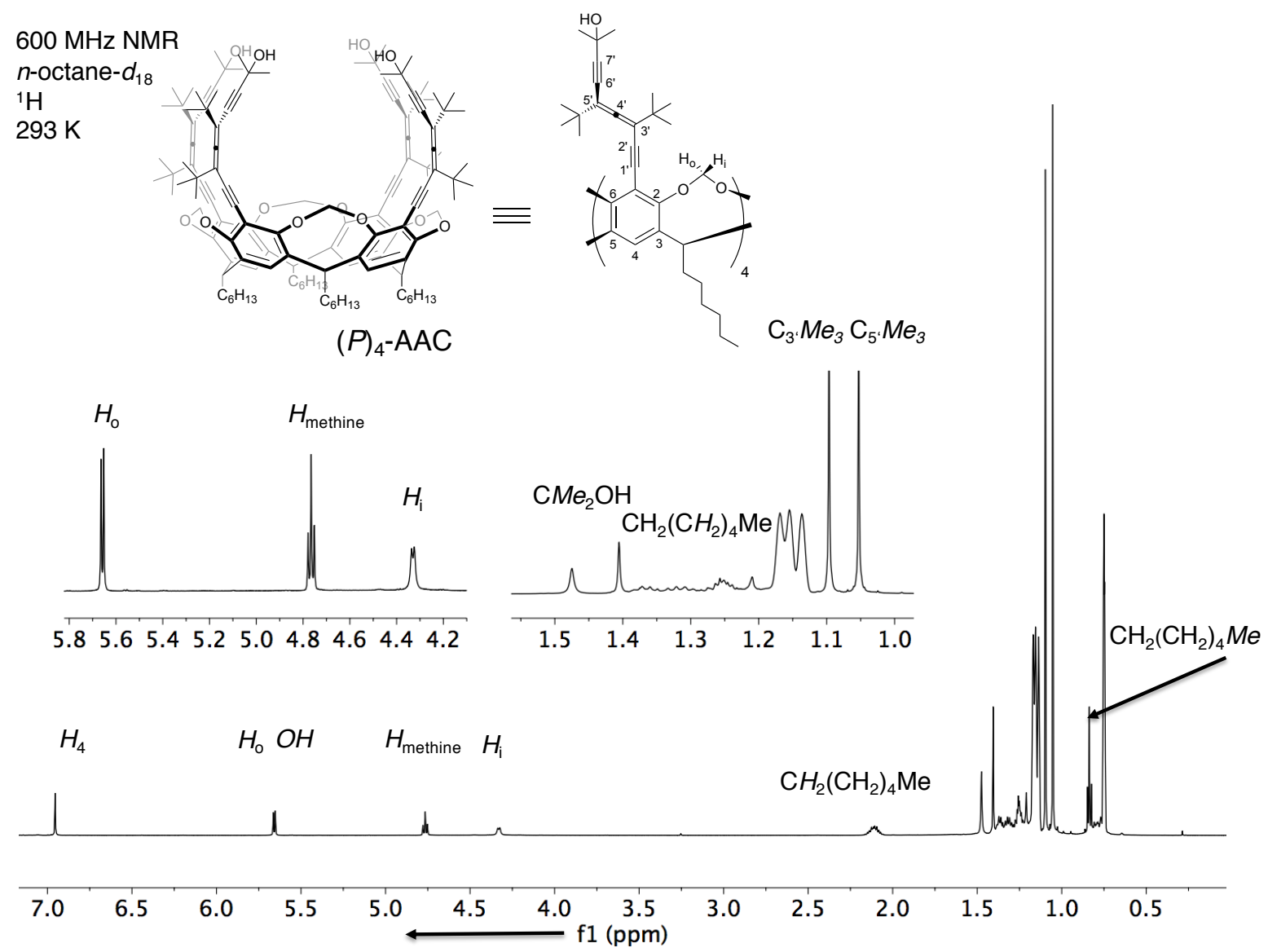

Figure S42. ${ }^{1} \mathrm{H}$ NMR $(600 \mathrm{MHz})$ traces of AAC $(P)_{4}-\mathbf{1}(6 \mathrm{mM})$ in [D $\left.\mathrm{D}_{18}\right]$-octane at $293 \mathrm{~K}$. Host-resonances: $\mathrm{H}_{\mathrm{i}}$ $=$ inside protons and $\mathrm{H}_{\mathrm{o}}=$ outside protons of the methylene bridge; $\mathrm{H}_{4}=$ aromatic protons, highlighted in Figure S41. 


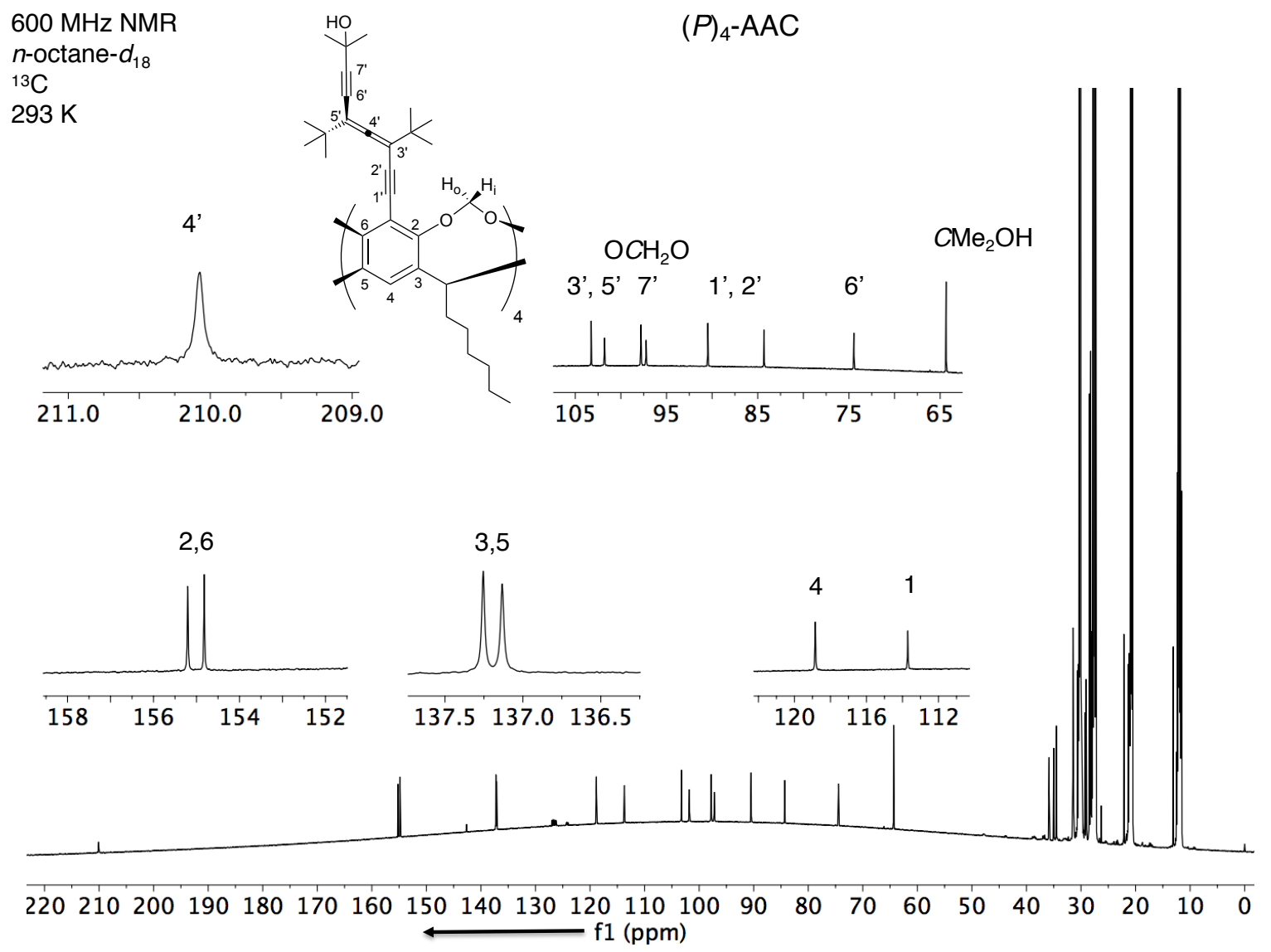

Figure S43. ${ }^{13} \mathrm{C}$ NMR $(150 \mathrm{MHz})$ traces of AAC $(P)_{4}-\mathbf{1}(6 \mathrm{mM})$ in $\left[\mathrm{D}_{18}\right] n$-octane at $293 \mathrm{~K}$. Corresponding hostresonances are highlighted in Figure S41. 
$600 \mathrm{MHz}$ NMR

$n$-octane- $d_{18}$

${ }^{1} \mathrm{H}$

$277 \mathrm{~K}$

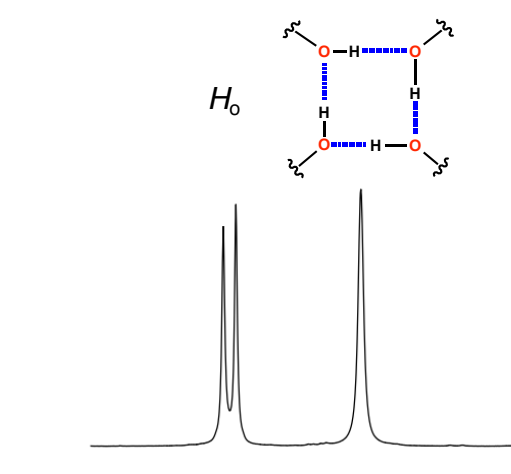

5.95 .95 .85 .85 .85 .75 .75 .6
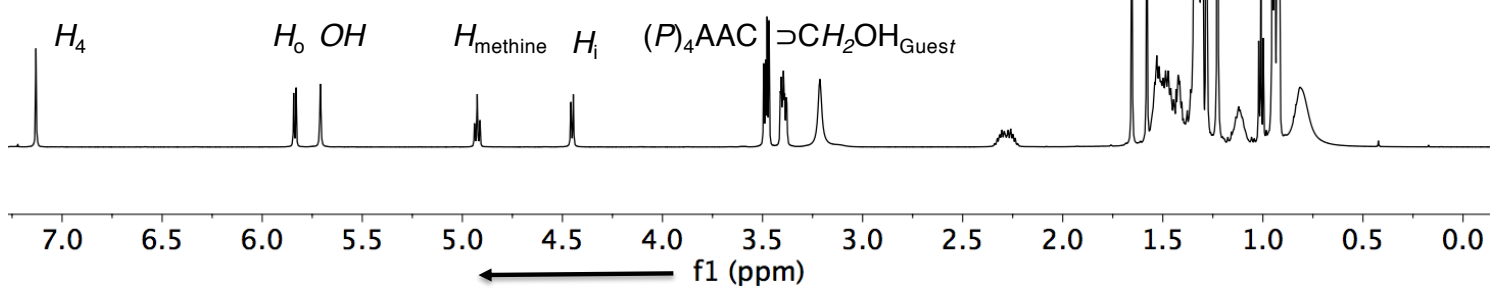

Figure S44. ${ }^{1} \mathrm{H}$ NMR $(600 \mathrm{MHz})$ traces of AAC $(P)_{4}-\mathbf{1}(7.0 \mathrm{mM})$ in $\left[\mathrm{D}_{18}\right] n$-octane at $277 \mathrm{~K}$ with 15 equiv. of $(R / S)-4$.. Host-resonances: $\mathrm{H}_{\mathrm{i}}=$ inside protons and $\mathrm{H}_{\mathrm{o}}=$ outside protons of the methylene bridge; $\mathrm{H}_{4}=$ aromatic protons, highlighted in Figure S41.
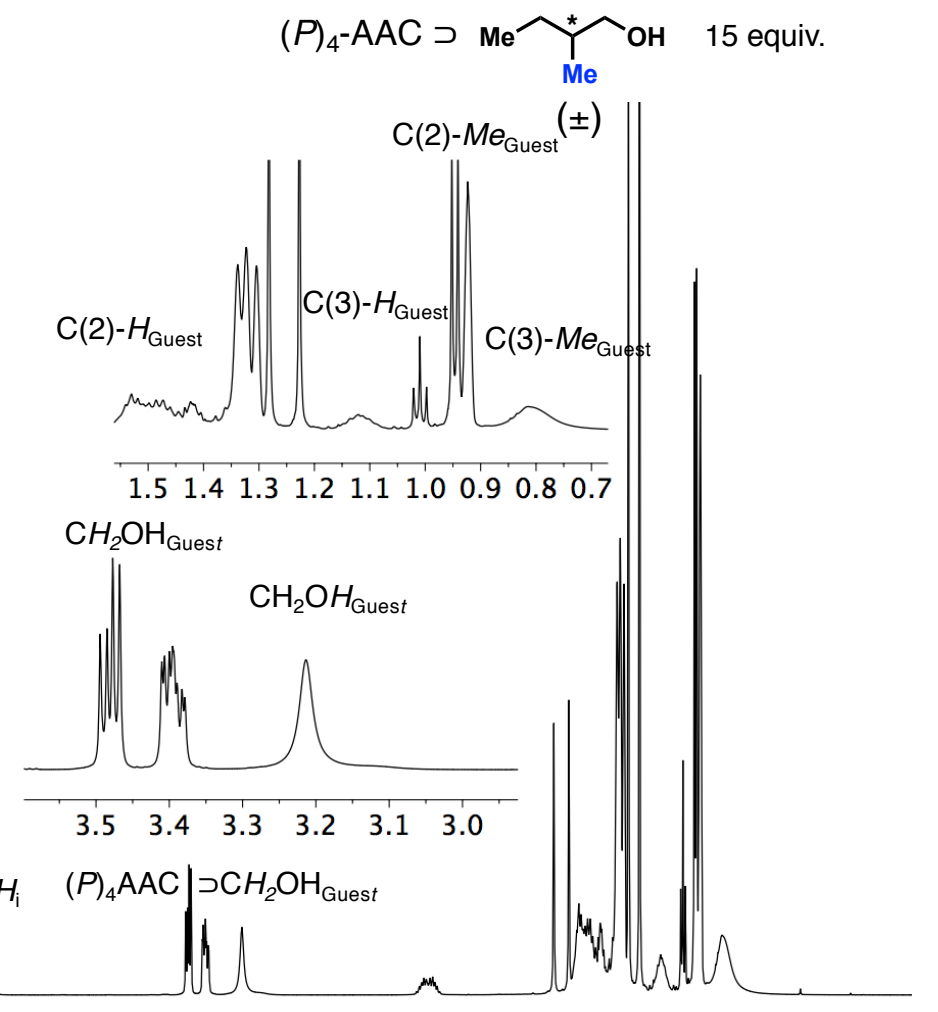

f1 (ppm) 
$600 \mathrm{MHz}$ NMR

$n$-octane- $d_{18}$

${ }^{13} \mathrm{C}$

$277 \mathrm{~K}$
$(P)_{4}$-AAC $\supset \mathrm{Me}_{\mathrm{Me}_{\mathrm{MeH}}}^{*} 15$ equiv.

$( \pm)$

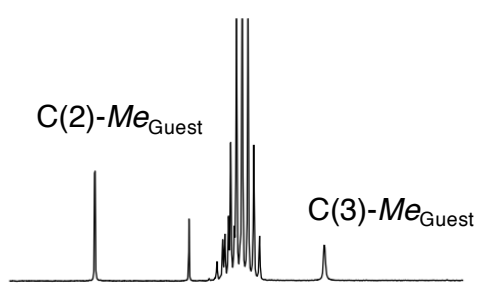

$\begin{array}{llllllllll}17 & 16 & 15 & 14 & 13 & 12 & 11 & 10 & 9 & 8\end{array}$
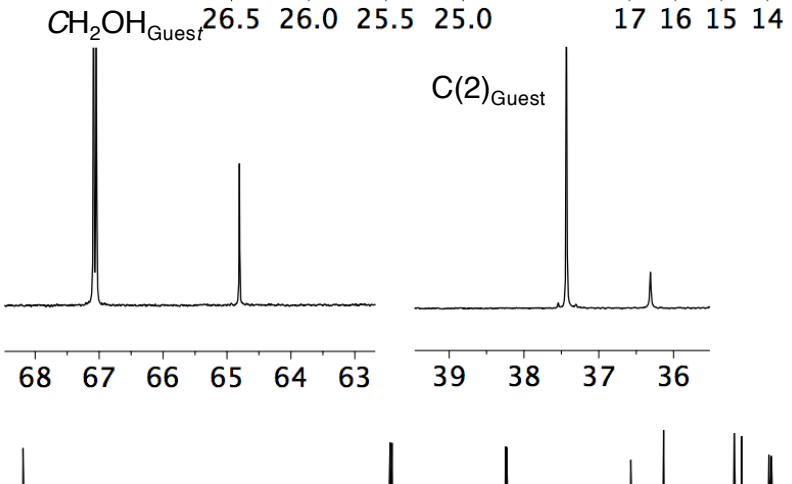

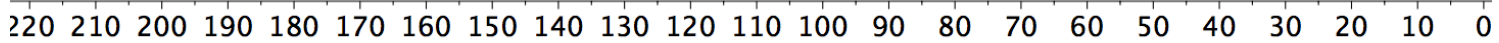
$\longleftarrow$ f1 (ppm)

Figure S45. ${ }^{13} \mathrm{C}$ NMR $(150 \mathrm{MHz})$ traces of AAC $(P)_{4}-\mathbf{1}(7.0 \mathrm{mM})$ in $\left[\mathrm{D}_{18}\right] n$-octane at $277 \mathrm{~K}$ with 15 equiv. of $(R / S)-4$. 
$600 \mathrm{MHz}$ NMR

$n$-octane- $d_{18}$

${ }^{1} \mathrm{H}$

$277 \mathrm{~K}$
$(P)_{4}$-AAC $\supset \mathrm{Me}^{\mathrm{Me}_{\text {Me }}^{*}} \mathrm{OH} 2$ equiv.

$( \pm)$

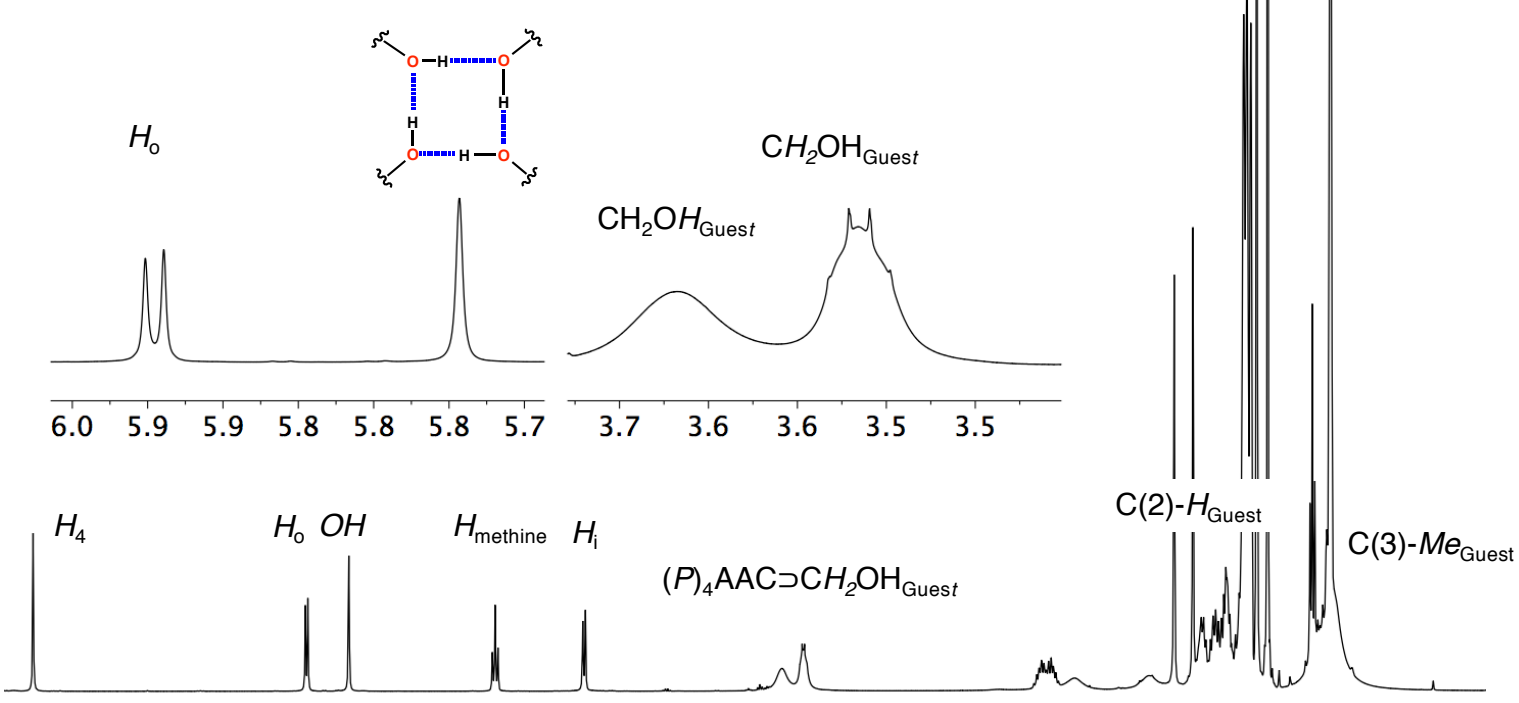
7.06
$\begin{array}{lll}6.5 & 6.0 & 5.5\end{array}$
$5.0 \quad 4.5$
4.0 (ppm)

Figure S46. ${ }^{1} \mathrm{H}$ NMR $(600 \mathrm{MHz})$ traces of AAC $(P)_{4}-\mathbf{1}(7.0 \mathrm{mM})$ in [ $\left.\mathrm{D}_{18}\right] n$-octane at $277 \mathrm{~K}$ with 2 equiv. of $(R / S)$ 6. Host-resonances: $\mathrm{H}_{\mathrm{i}}=$ inside protons and $\mathrm{H}_{\mathrm{o}}=$ outside protons of the methylene bridge; $\mathrm{H}_{4}=$ aromatic protons, highlighted in Figure S41. 


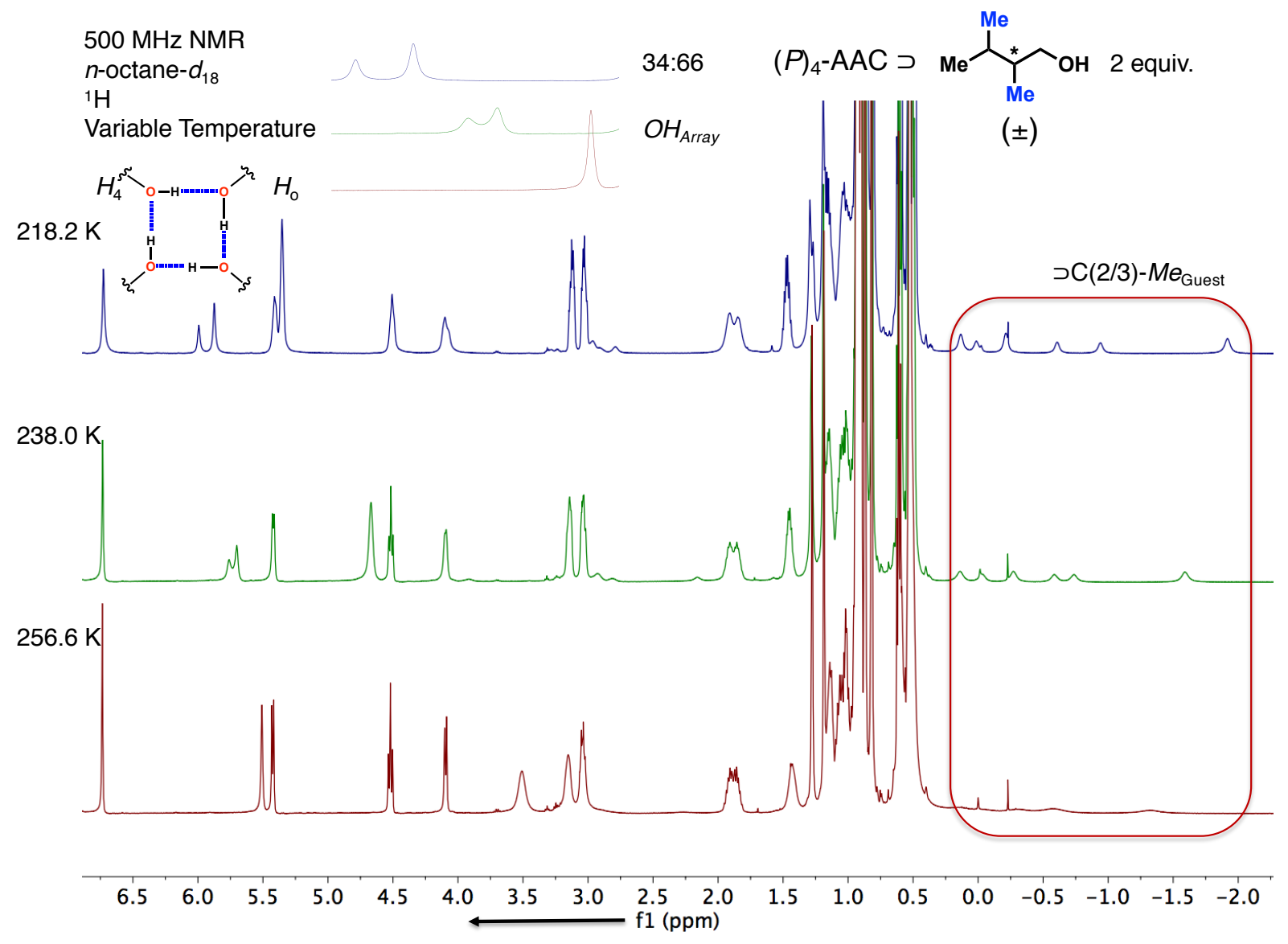

Figure S47. VT- ${ }^{1} \mathrm{H}$ NMR $(500 \mathrm{MHz})$ traces of AAC $(P)_{4}-1(7.0 \mathrm{mM})$ in $\left[\mathrm{D}_{18}\right] n$-octane at $277 \mathrm{~K}$ with 2 equiv. of $(R / S)$-6. Host-resonances: $\mathrm{H}_{\mathrm{i}}=$ inside protons and $\mathrm{H}_{\mathrm{o}}=$ outside protons of the methylene bridge; $\mathrm{H}_{4}=$ aromatic protons, highlighted in Figure S41. Splitting of the Host-OH-resonances correspond to the diastereoisomeric complexes of the host-guest complexes. 
$600 \mathrm{MHz}$ NMR

$n$-octane- $d_{18}$

${ }^{13} \mathrm{C}$

$277 \mathrm{~K}$
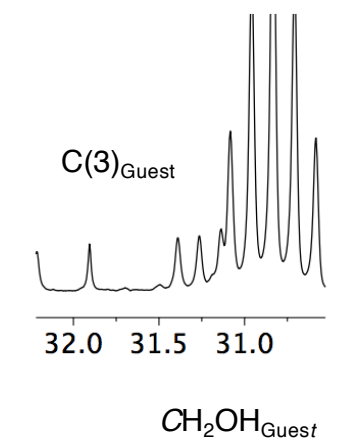

$\mathrm{C}(2 / 3)-M e_{\text {Guest }}$
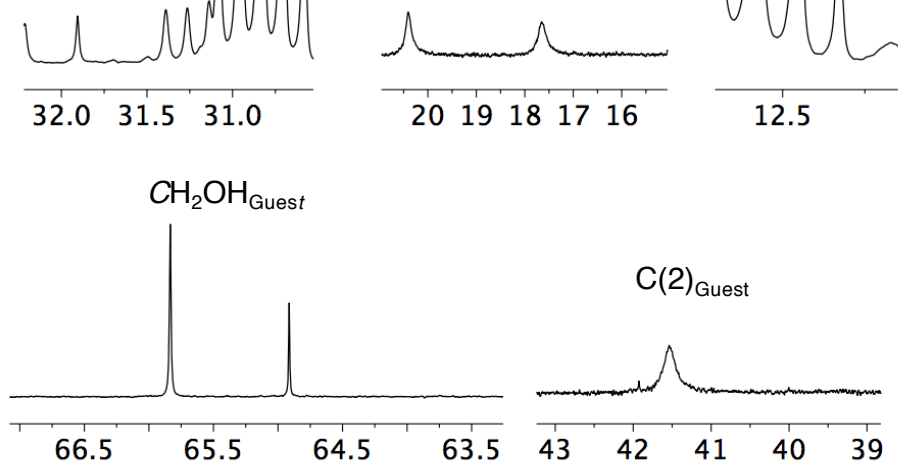

66.5

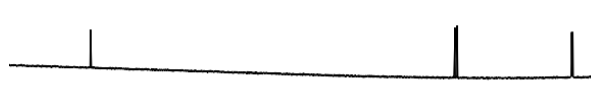

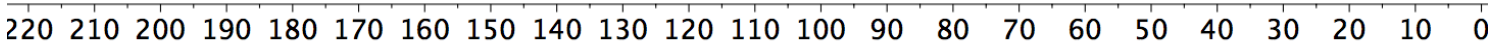

Figure S48. ${ }^{13} \mathrm{C}$ NMR $(150 \mathrm{MHz})$ traces of $\mathrm{AAC}(P)_{4}-\mathbf{1}(7.0 \mathrm{mM})$ in $\left[\mathrm{D}_{18}\right] n$-octane at $277 \mathrm{~K}$ with 2 equiv. of $(R / S)-6$.

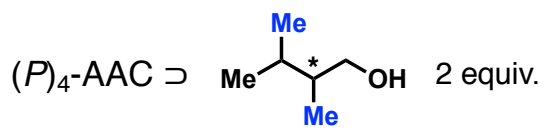

$( \pm)$
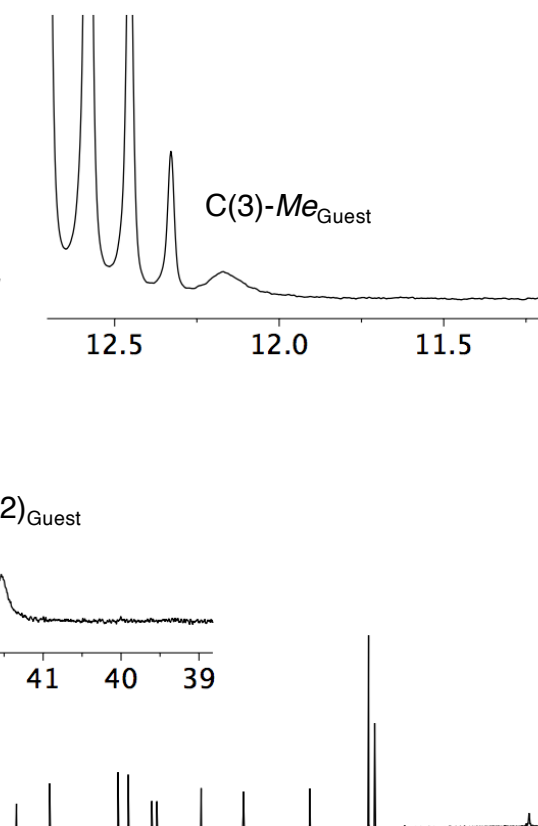

f1 (ppm) 
$600 \mathrm{MHz}$ NMR

$n$-octane- $d_{18}$

${ }^{1} \mathrm{H}$

$277 \mathrm{~K}$
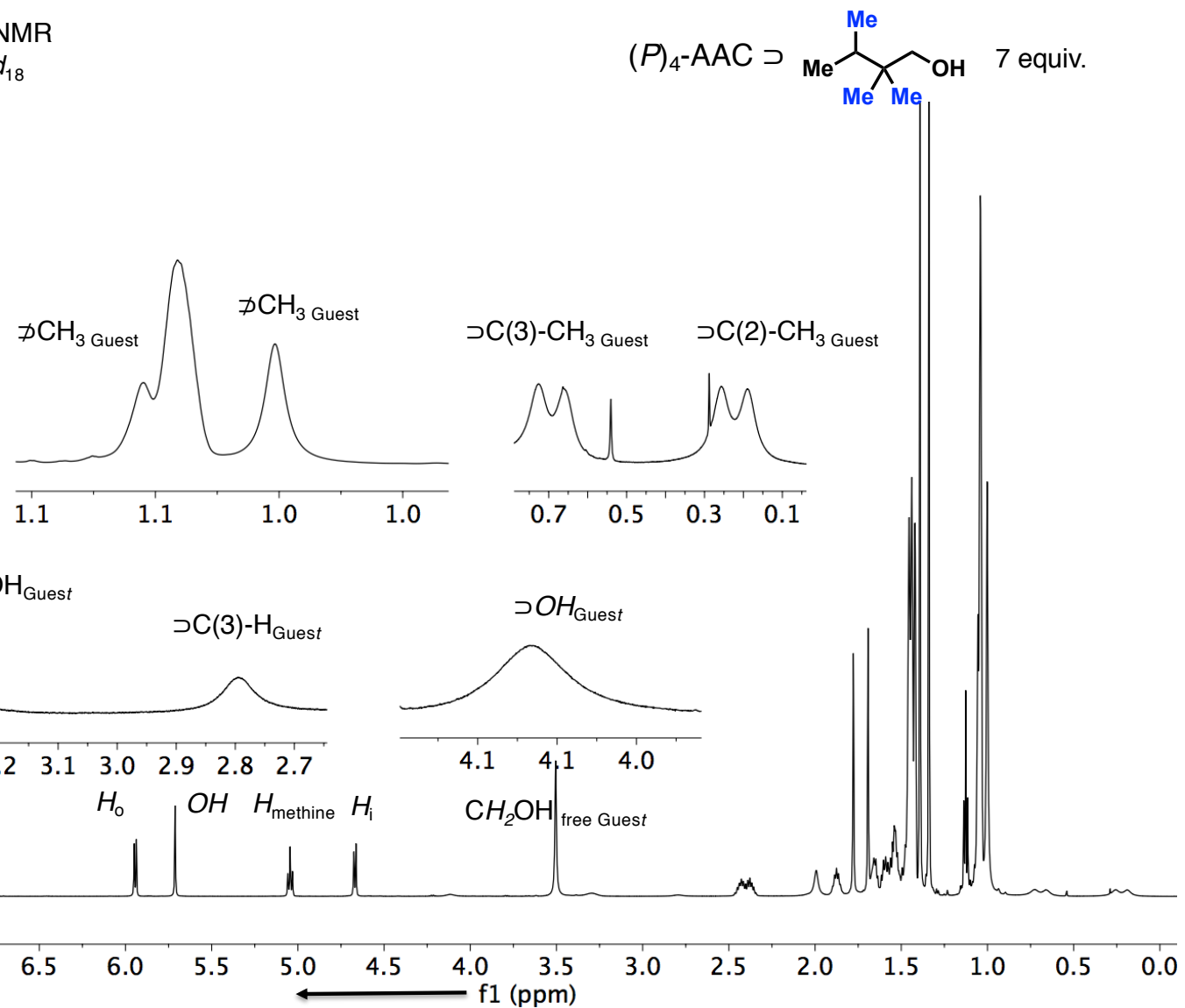

Figure S49. ${ }^{1} \mathrm{H}$ NMR $(600 \mathrm{MHz})$ traces of AAC $(P)_{4}-1(7.0 \mathrm{mM})$ in $\left[\mathrm{D}_{18}\right] n$-octane at $277 \mathrm{~K}$ with 7 equiv. of 9. The $\supset$ denotes the complexed guest and $D$ signifies the unbound guest molecule. Host-resonances: $H_{i}=$ inside protons and $\mathrm{H}_{\mathrm{o}}=$ outside protons of the methylene bridge; $\mathrm{H}_{4}=$ aromatic protons, highlighted in Figure S41. 


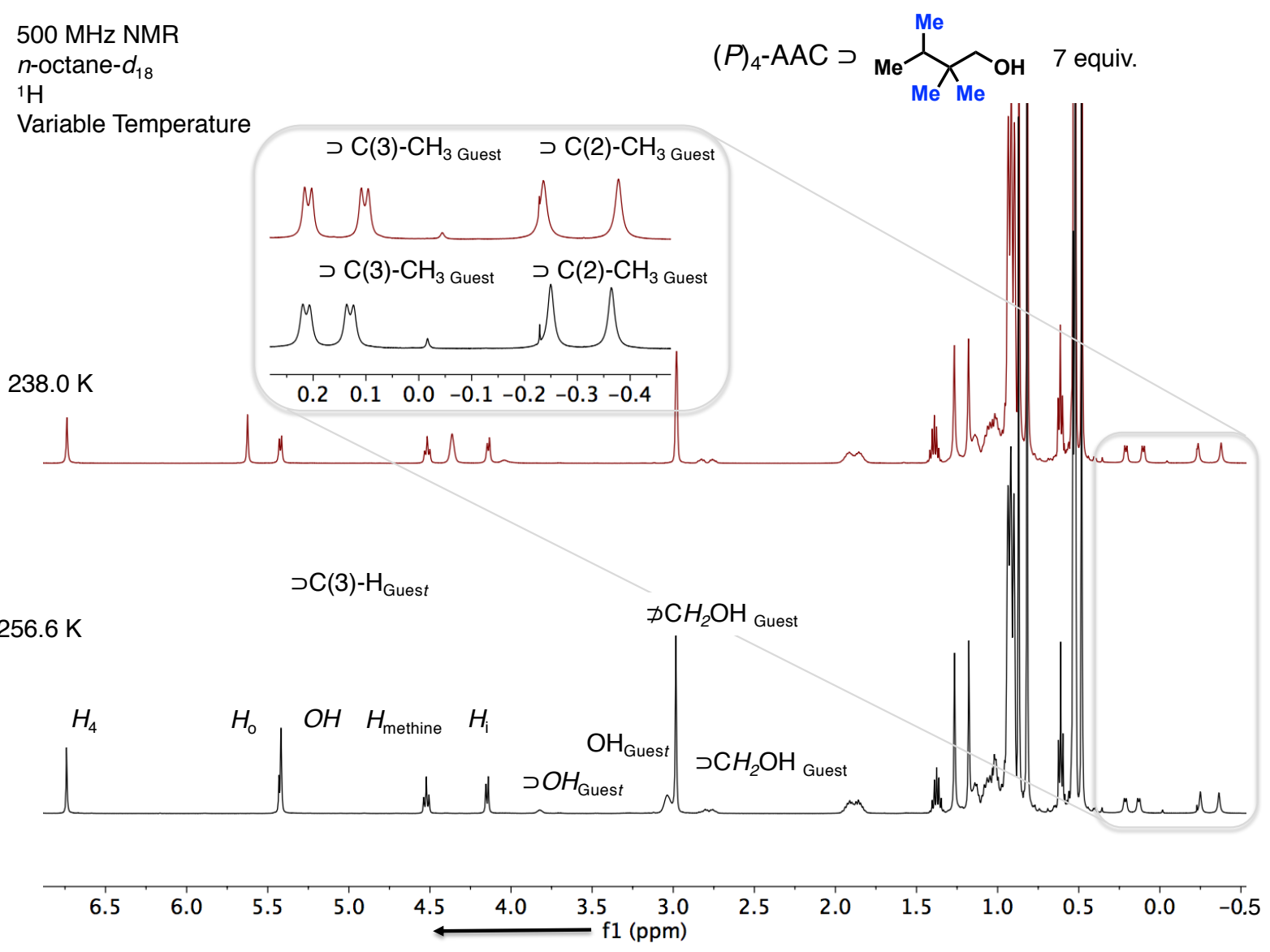

Figure S50. VT- ${ }^{1} \mathrm{H}$ NMR $(500 \mathrm{MHz})$ traces of AAC $(P)_{4}-1$ ( $\left.7.0 \mathrm{mM}\right)$ in $\left[\mathrm{D}_{18}\right] n$-octane at $277 \mathrm{~K}$ with 7 equiv. of 9. Host-resonances: $\mathrm{H}_{\mathrm{i}}=$ inside protons and $\mathrm{H}_{\mathrm{o}}=$ outside protons of the methylene bridge; $\mathrm{H}_{4}=$ aromatic protons, highlighted in Figure S41. 


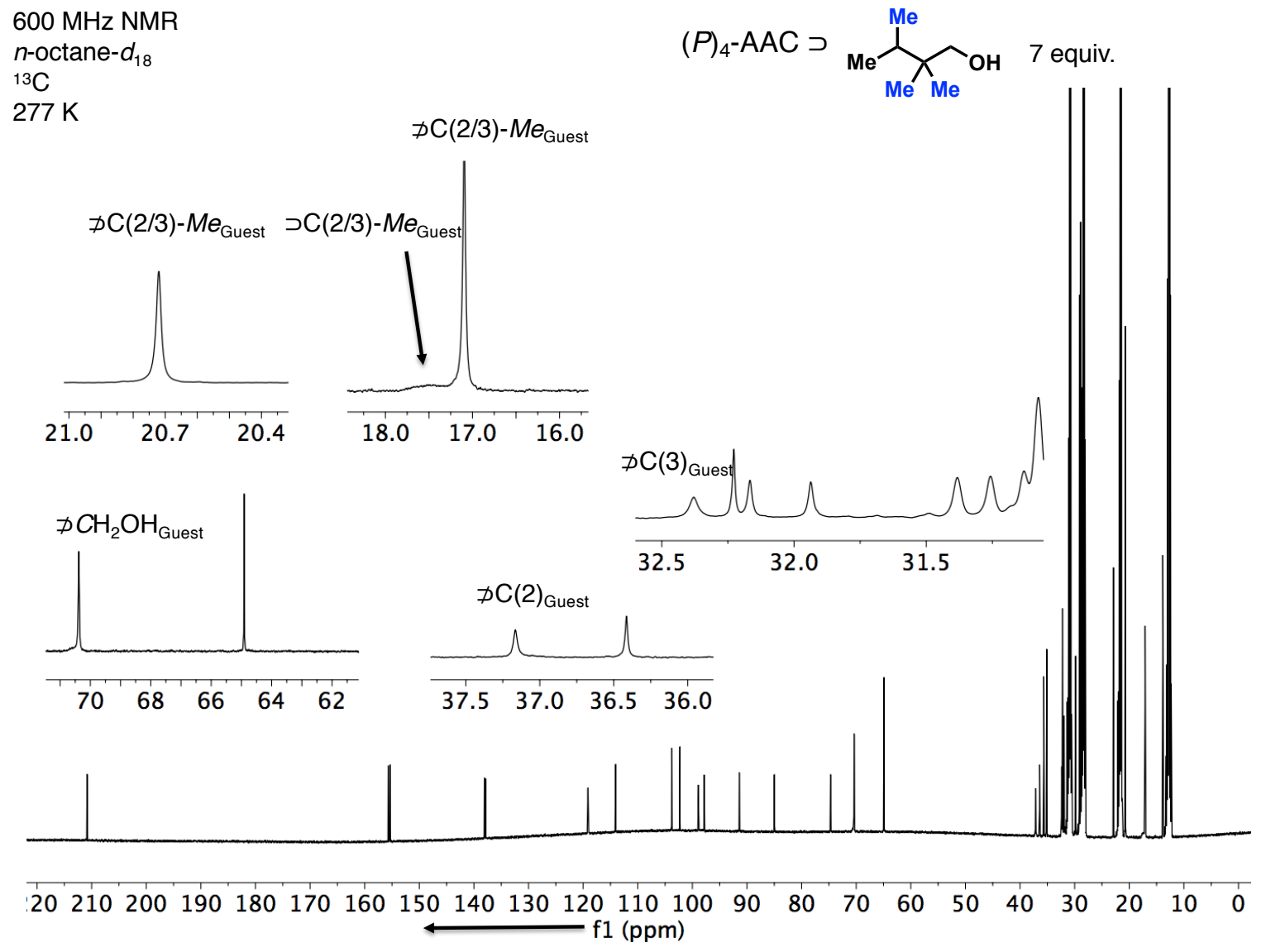

Figure S51. ${ }^{13} \mathrm{C}$ NMR $(150 \mathrm{MHz})$ traces of AAC $(P)_{4}-\mathbf{1}(7.0 \mathrm{mM})$ in $\left[\mathrm{D}_{18}\right] n$-octane at $277 \mathrm{~K}$ with 7 equiv. of $\mathbf{9}$. 
$600 \mathrm{MHz}$ NMR

$n$-octane- $d_{18}$

${ }^{1} \mathrm{H}$

$277 \mathrm{~K}$

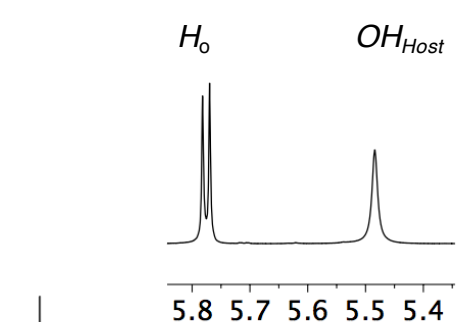

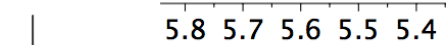

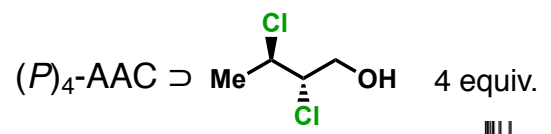

$( \pm)$

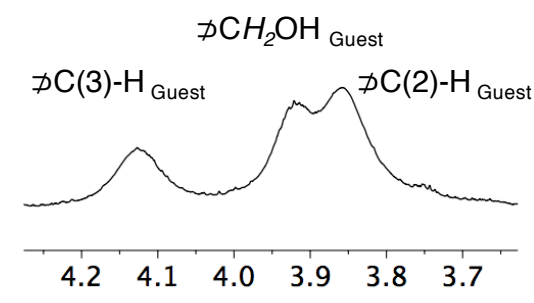

$\begin{array}{llll}H_{4} & H_{0} & O H & H_{\text {methine }} \\ H_{\mathrm{i}}\end{array}$
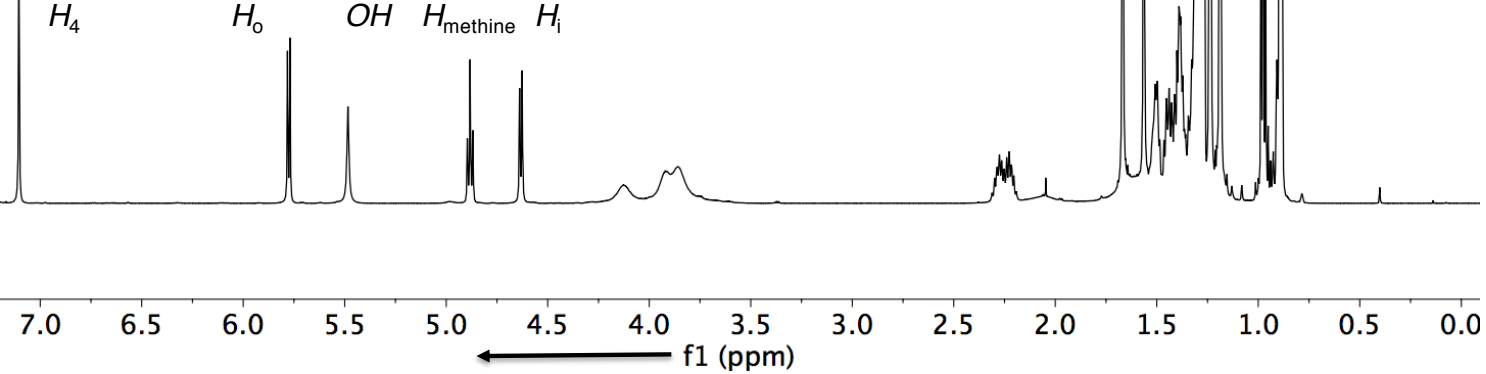

Figure S52. ${ }^{1} \mathrm{H}$ NMR $(600 \mathrm{MHz})$ traces of AAC $(P)_{4}-1(7.0 \mathrm{mM})$ in $\left[\mathrm{D}_{18}\right] n$-octane at $277 \mathrm{~K}$ with 4 equiv. of $\left(R^{*}, S^{*}\right)-7$. The $D$ signifies the unbound guest molecule at fast exchange on the NMR-timescale. Host-resonances: $\mathrm{H}_{\mathrm{i}}=$ inside protons and $\mathrm{H}_{\mathrm{o}}=$ outside protons of the methylene bridge; $\mathrm{H}_{4}=$ aromatic protons, highlighted in Figure S41. 
$600 \mathrm{MHz}$ NMR

$n$-octane- $d_{18}$

${ }^{13} \mathrm{C}$

$277 \mathrm{~K}$

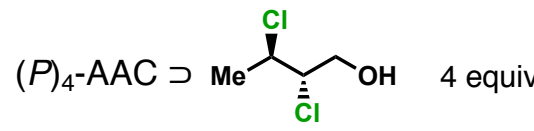

$( \pm)$
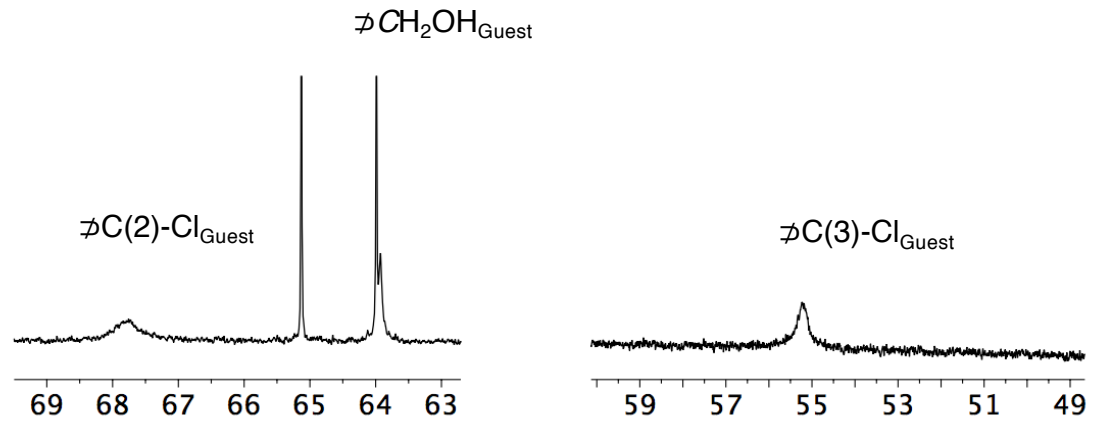

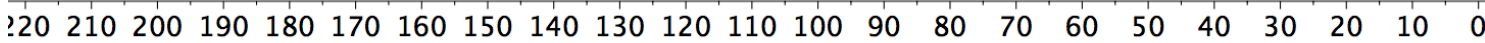
$\longleftarrow$ f1 (ppm)

Figure S53. ${ }^{13} \mathrm{C}$ NMR $(150 \mathrm{MHz})$ traces of AAC $(P)_{4}-1(7.0 \mathrm{mM})$ in $\left[\mathrm{D}_{18}\right]$-octane at $277 \mathrm{~K}$ with 4 equiv. of $\left(R^{*}, S^{*}\right)-7$. The $D$ signifies the unbound guest molecule at fast exchange on the NMR-timescale. 
$600 \mathrm{MHz}$ NMR

$n$-octane- $d_{18}$

${ }^{1} \mathrm{H}$

$277 \mathrm{~K}$

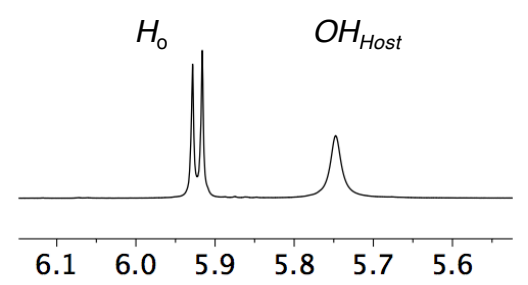

$(P)_{4}-\mathrm{AAC} \supset \mathrm{Me}_{\mathrm{Cl}}^{\mathrm{Cl}} \mathrm{OH} 4$ equiv.

$( \pm)$
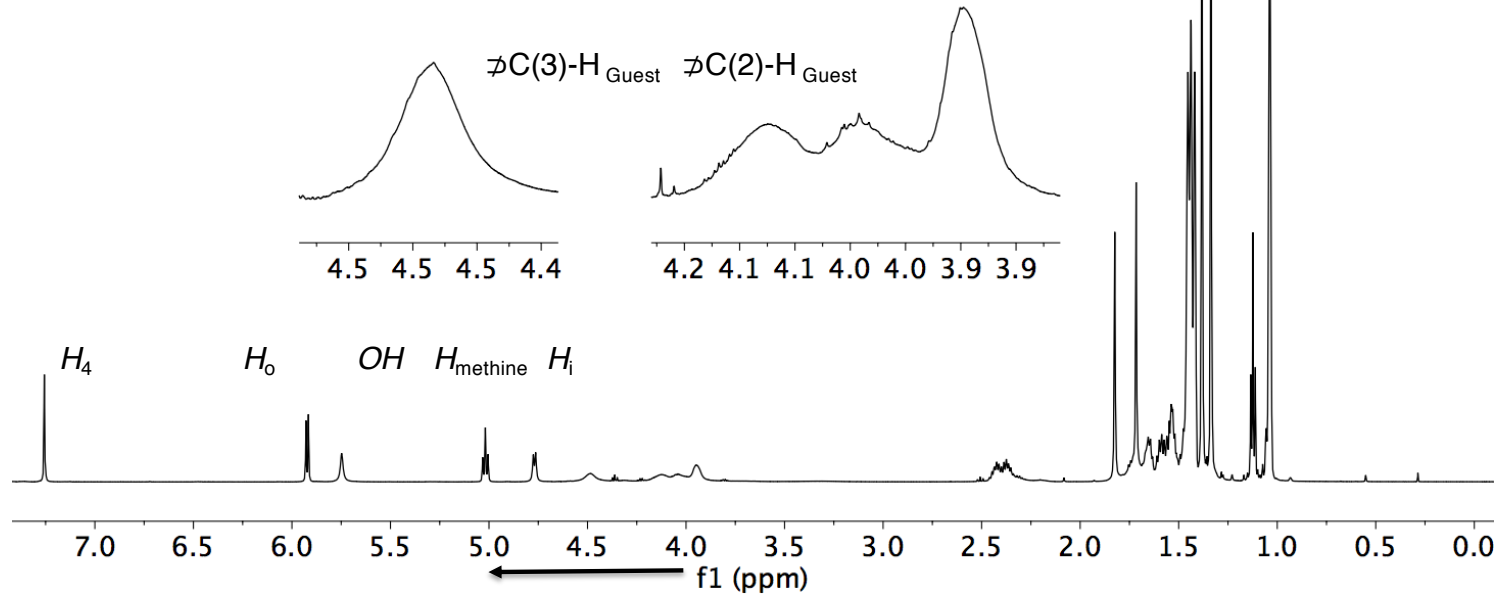

Figure S54. ${ }^{1} \mathrm{H}$ NMR $(600 \mathrm{MHz})$ traces of AAC $(P)_{4}-1(7.0 \mathrm{mM})$ in $\left[\mathrm{D}_{18}\right] n$-octane at $277 \mathrm{~K}$ with 4 equiv. of $\left(R^{*}, R^{*}\right)-7$. The $D$ signifies the unbound guest molecule at fast exchange on the NMR-timescale. Host-resonances: $\mathrm{H}_{\mathrm{i}}=$ inside protons and $\mathrm{H}_{\mathrm{o}}=$ outside protons of the methylene bridge; $\mathrm{H}_{4}=$ aromatic protons, highlighted in Figure S41. 
$600 \mathrm{MHz}$ NMR

$n$-octane- $d_{18}$

${ }^{13} \mathrm{C}$

$277 \mathrm{~K}$

$\begin{array}{lllllll}68 & 67 & 66 & 65 & 64 & 63 & 62\end{array}$

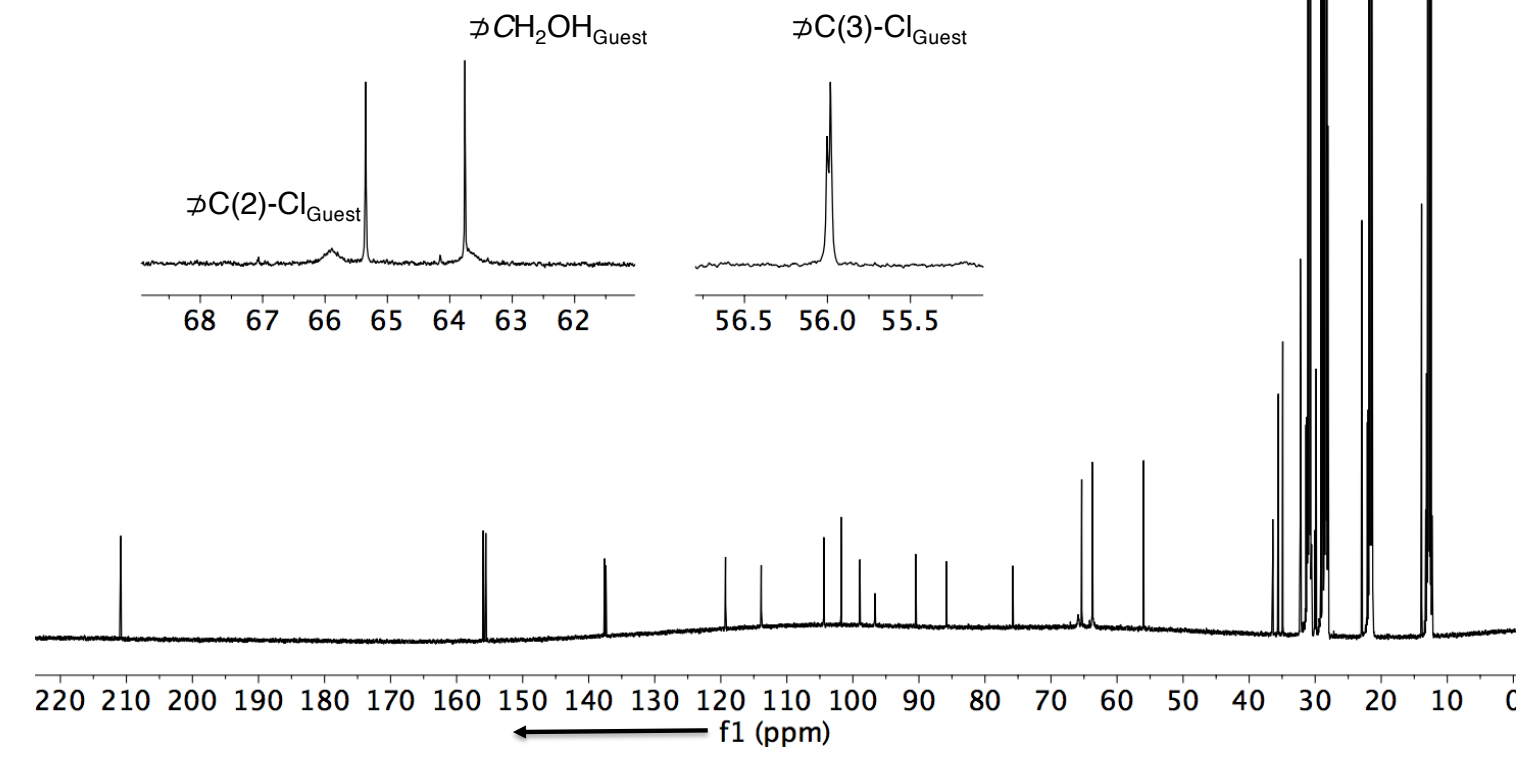

$(P)_{4}$-AAC $\supset \mathrm{Me}_{\mathrm{Cl}_{\mathrm{l}}}^{\mathrm{Cl}} \mathrm{OH} 4$ equiv.

$( \pm)$

Figure S55. ${ }^{13} \mathrm{C}$ NMR $(150 \mathrm{MHz})$ traces of $\mathrm{AAC}(P)_{4}-\mathbf{1}(7.0 \mathrm{mM})$ in $\left[\mathrm{D}_{18}\right] n$-octane at $277 \mathrm{~K}$ with 4 equiv. of $\left(R^{*}, R^{*}\right)-7$. The $D$ signifies the unbound guest molecule at fast exchange on the NMR-timescale. 
$600 \mathrm{MHz}$ NMR

$n$-octane- $d_{18}$

${ }^{1} \mathrm{H}$

$277 \mathrm{~K}$

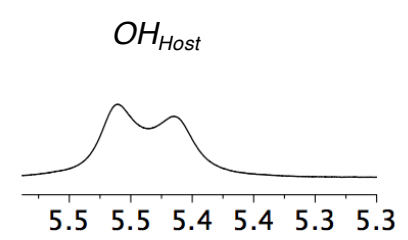
$\not \supset \mathrm{CH}_{2} \mathrm{OH}_{\text {Guest }}$
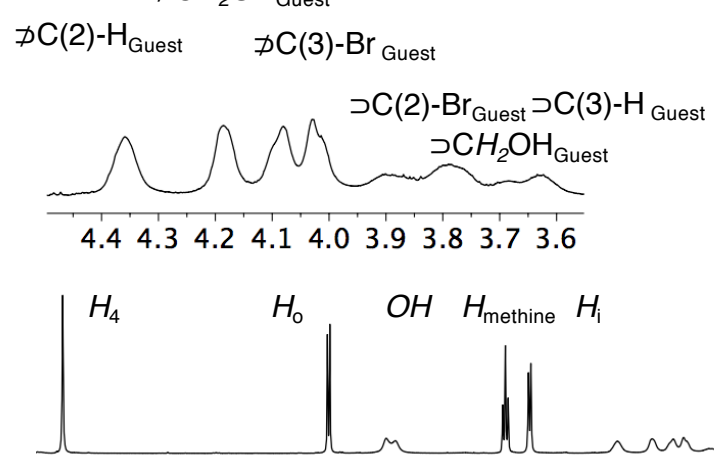

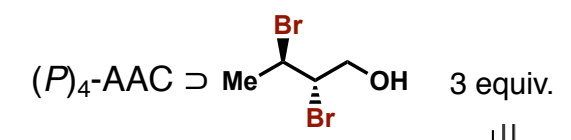

$( \pm)$
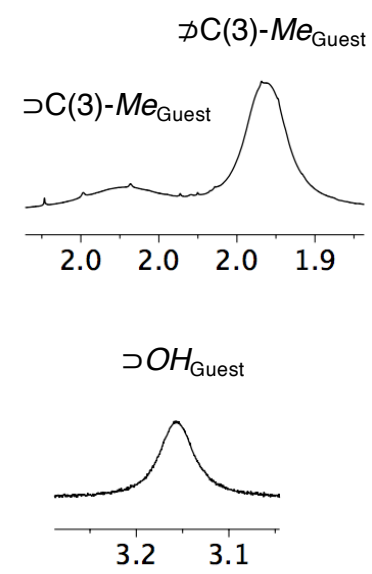

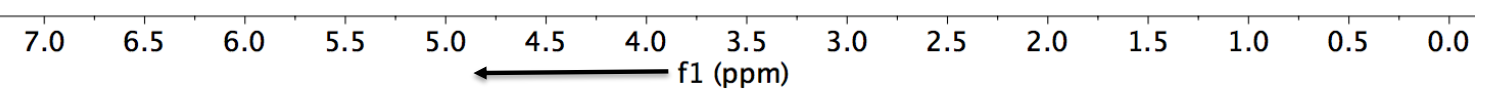

Figure S56. ${ }^{1} \mathrm{H}$ NMR $(600 \mathrm{MHz})$ traces of AAC $(P)_{4}-1(7.0 \mathrm{mM})$ in $\left[\mathrm{D}_{18}\right] n$-octane at $277 \mathrm{~K}$ with 3 equiv. of $\left(R^{*}, S^{*}\right)-8$. The $\supset$ denotes the complexed guest and $D$ signifies the unbound at slow exchange on the NMRtimescale. Host-resonances: $\mathrm{H}_{\mathrm{i}}=$ inside protons and $\mathrm{H}_{\mathrm{o}}=$ outside protons of the methylene bridge; $\mathrm{H}_{4}=$ aromatic protons, highlighted in Figure S41. 
$600 \mathrm{MHz}$ NMR

$n$-octane- $d_{18}$

${ }^{13} \mathrm{C}$

$277 \mathrm{~K}$

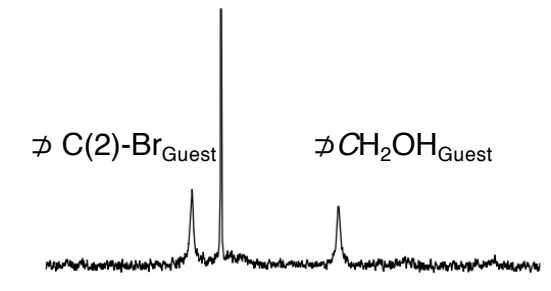

$\begin{array}{llllllllll}68 & 67 & 66 & 65 & 64 & 63 & 62 & 61 & 60 & 59\end{array}$

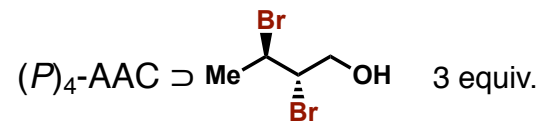

$( \pm)$

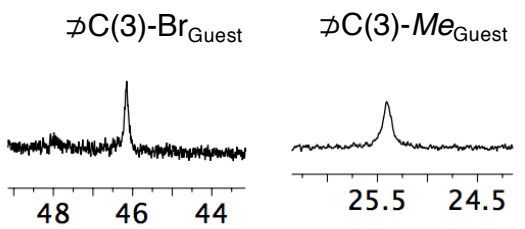

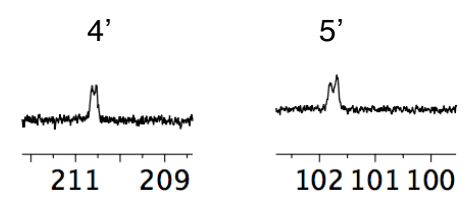

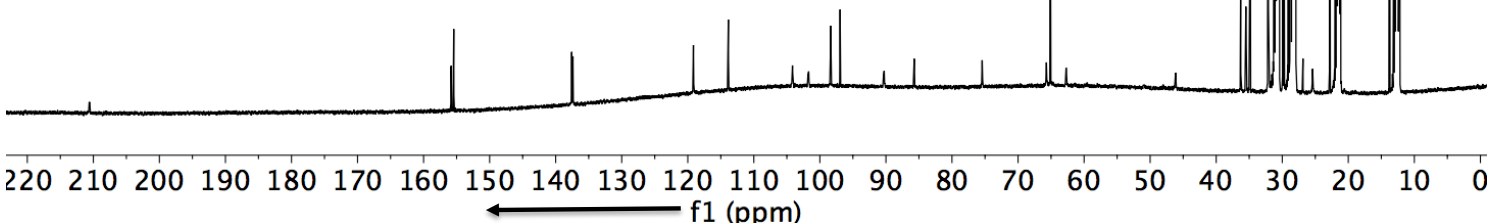

Figure S57. ${ }^{13} \mathrm{C}$ NMR $(150 \mathrm{MHz})$ traces of $\mathrm{AAC}(P)_{4}-\mathbf{1}(7.0 \mathrm{mM})$ in $\left[\mathrm{D}_{18}\right] n$-octane at $277 \mathrm{~K}$ with 3 equiv. of $\left(R^{*}, S^{*}\right)-8$. The $D$ signifies the unbound guest molecule. 


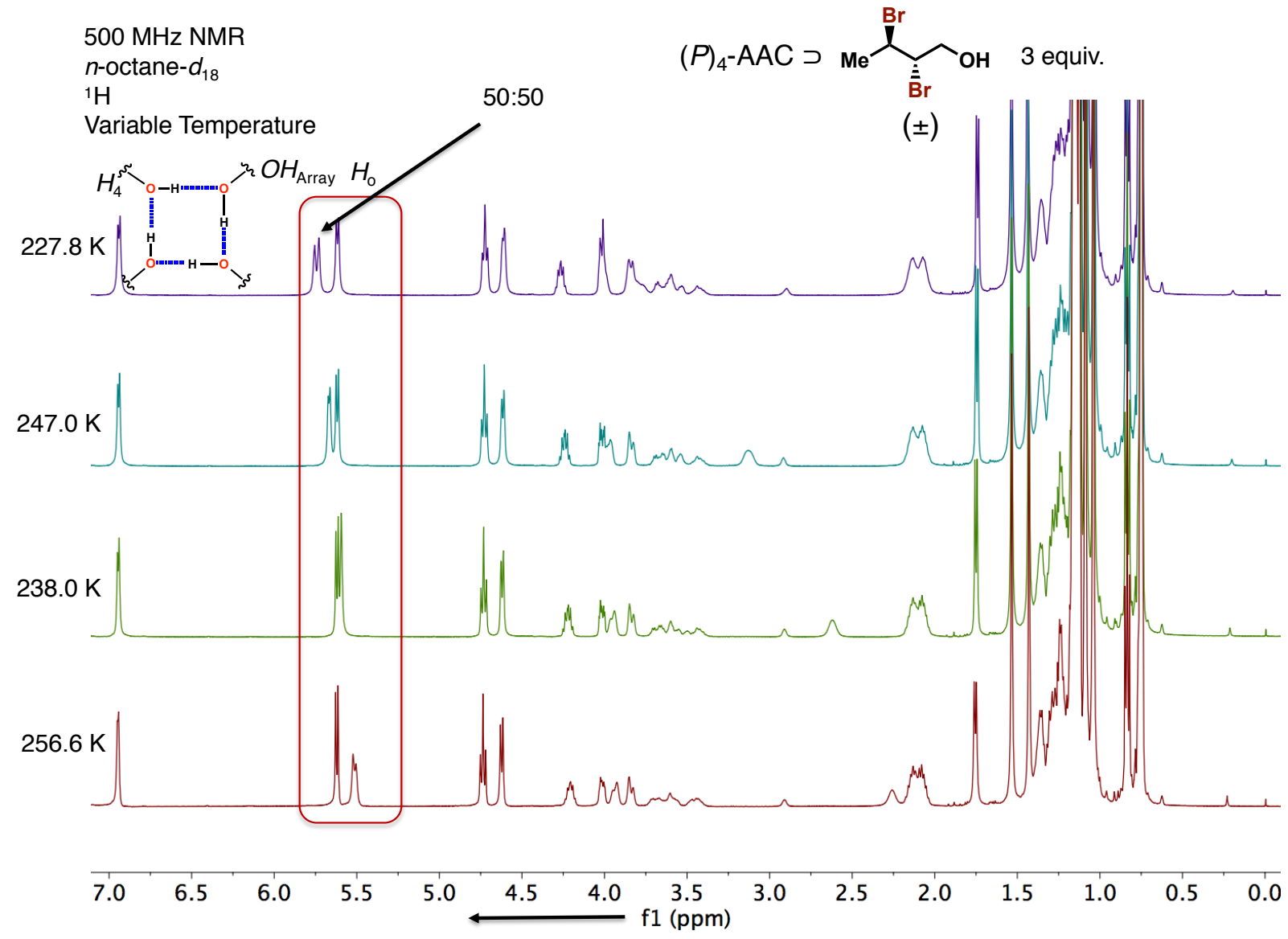

Figure S58. VT- ${ }^{1} \mathrm{H}$ NMR $(500 \mathrm{MHz})$ traces of AAC $(P)_{4}-1(7.0 \mathrm{mM})$ in $\left[\mathrm{D}_{18}\right] n$-octane at $277 \mathrm{~K}$ with 3 equiv. of $\left(R^{*}, S^{*}\right)$-8. Host-resonances: $\mathrm{H}_{\mathrm{i}}=$ inside protons and $\mathrm{H}_{\mathrm{o}}=$ outside protons of the methylene bridge; $\mathrm{H}_{4}=$ aromatic protons, highlighted in Figure S41. Splitting of the Host-OH-resonances correspond to the diastereoisomeric complexes of the host-guest complexes. 
$600 \mathrm{MHz} N M R$

$n$-octane- $d_{18}$

${ }^{1} \mathrm{H}$

$277 \mathrm{~K}$

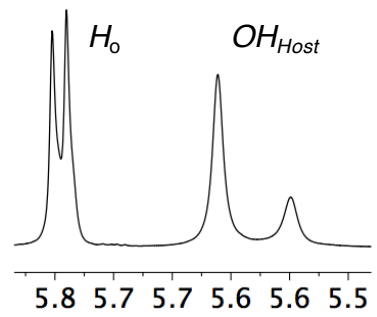

$(P)_{4}$-AAC $\supset \mathrm{Me}^{\mathrm{Br}} \mathrm{OH} 3$ equiv.

(士)

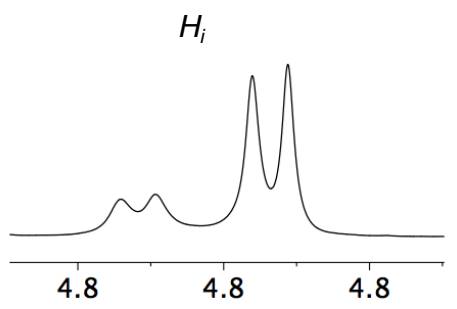

$\not \supset \mathrm{C}(3)-M e_{\text {Guest }}$

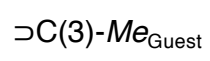

$\not \supset \mathrm{C}(2)-\mathrm{H}_{\text {Guest }}$

$\not \supset \mathrm{C}(3)-\mathrm{H}_{\text {Guest }} \stackrel{\supset \mathrm{C}(3)-\mathrm{H}_{\text {Guest }}}{\not \supset \mathrm{CH}_{2} \mathrm{OH}_{\text {Guest }}}$

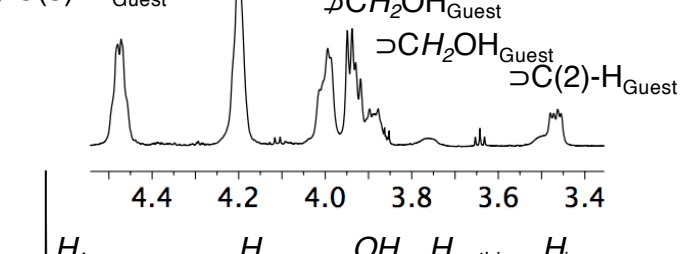

$\supset \mathrm{CH}_{2} \mathrm{OH}_{\mathrm{G}} \supset \mathrm{OH}_{\text {Guest }}$
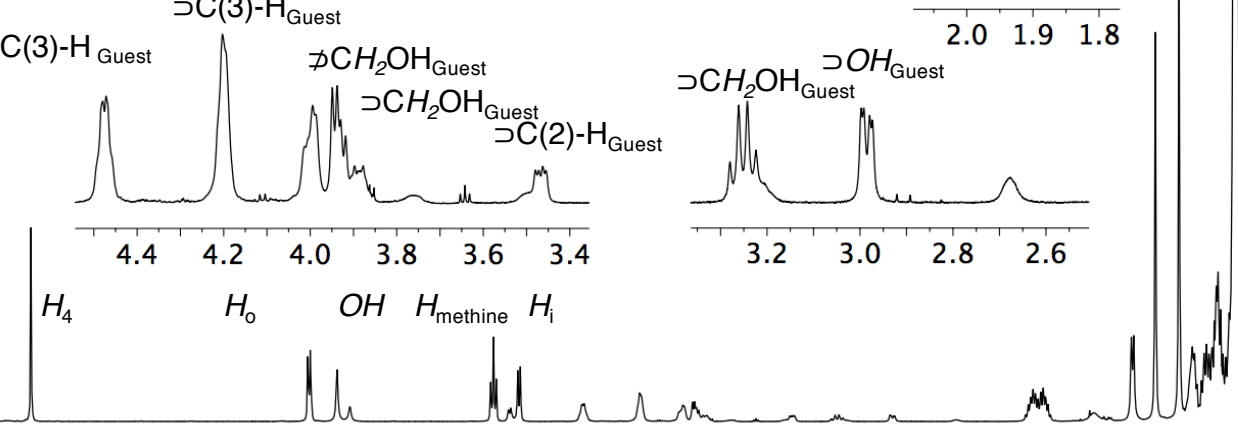

$\begin{array}{lllllll}7.0 & 6.5 & 6.0 & 5.5 & 5.0 & 4.5 & 4.0\end{array}$

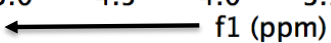

Figure S59. ${ }^{1} \mathrm{H}$ NMR $(600 \mathrm{MHz})$ traces of AAC $(P)_{4}-1(7.0 \mathrm{mM})$ in $\left[\mathrm{D}_{18}\right] n$-octane at $277 \mathrm{~K}$ with 3 equiv. of $\left(R^{*}, R^{*}\right)-8$. The $\supset$ denotes the complexed guest and $D$ signifies the unbound at slow exchange on the NMRtimescale. Host-resonances: $\mathrm{H}_{\mathrm{i}}=$ inside protons and $\mathrm{H}_{\mathrm{o}}=$ outside protons of the methylene bridge; $\mathrm{H}_{4}=$ aromatic protons, highlighted in Figure S41. 
$600 \mathrm{MHz}$ NMR

$n$-octane- $d_{18}$

${ }^{1} \mathrm{H}$

$277 \mathrm{~K}$
$(P)_{4}$-AAC $\supset \mathrm{Me}_{\mathrm{Br}^{\mathrm{Br}}}^{\mathrm{Br}} 1.5$ equiv.

$(R, R)$

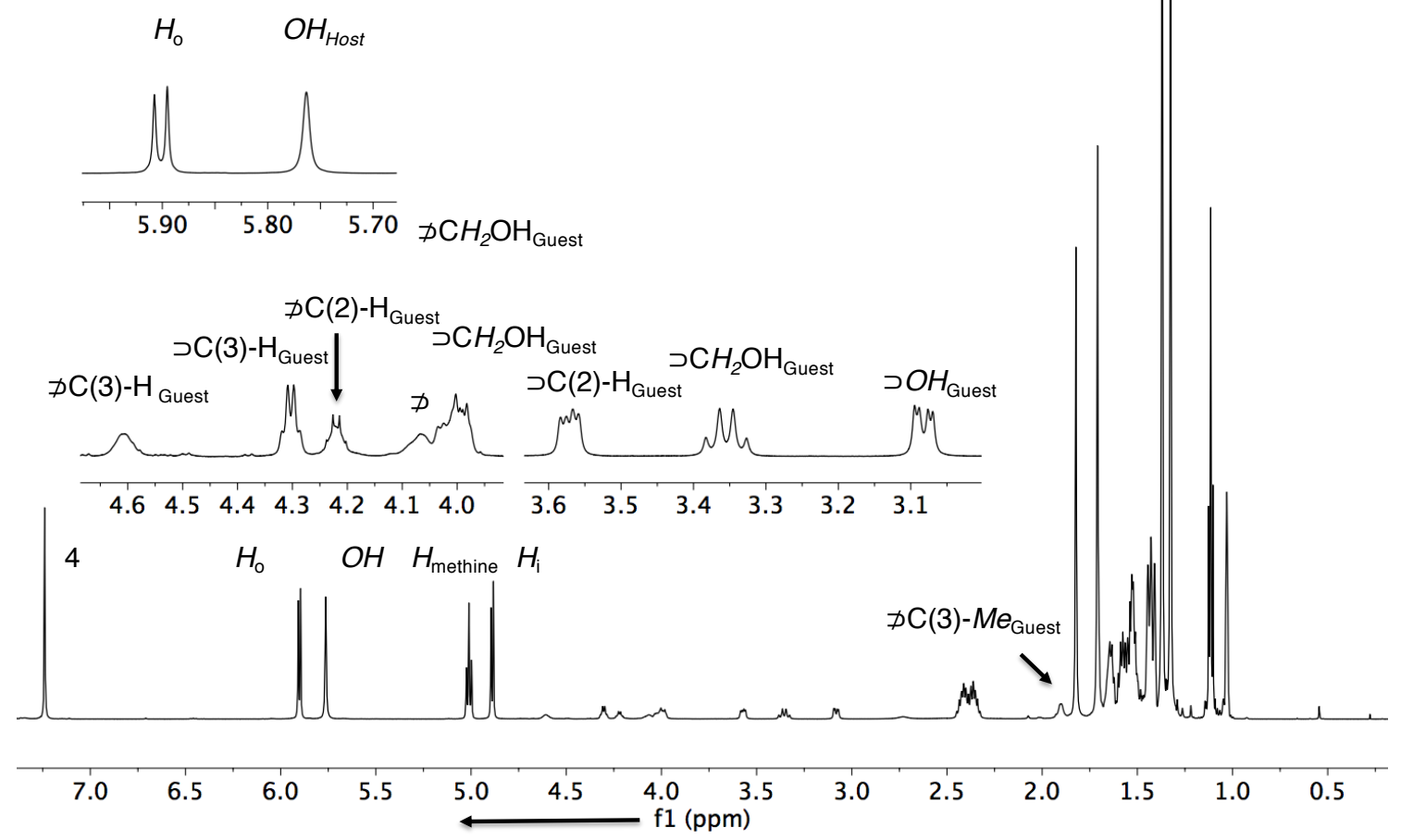

Figure S60. ${ }^{1} \mathrm{H}$ NMR $(600 \mathrm{MHz})$ traces of AAC $(P)_{4}-1(20.0 \mathrm{mM})$ in $\left[\mathrm{D}_{18}\right] n$-octane at $277 \mathrm{~K}$ with 1.5 equiv. of the enantiopure $(R, R)-8$. The $\supset$ denotes the complexed guest and $D$ signifies the unbound at slow exchange on the NMR-timescale. Host-resonances: $\mathrm{H}_{\mathrm{i}}=$ inside protons and $\mathrm{H}_{\mathrm{o}}=$ outside protons of the methylene bridge; $\mathrm{H}_{4}$ $=$ aromatic protons, highlighted in Figure S41. 


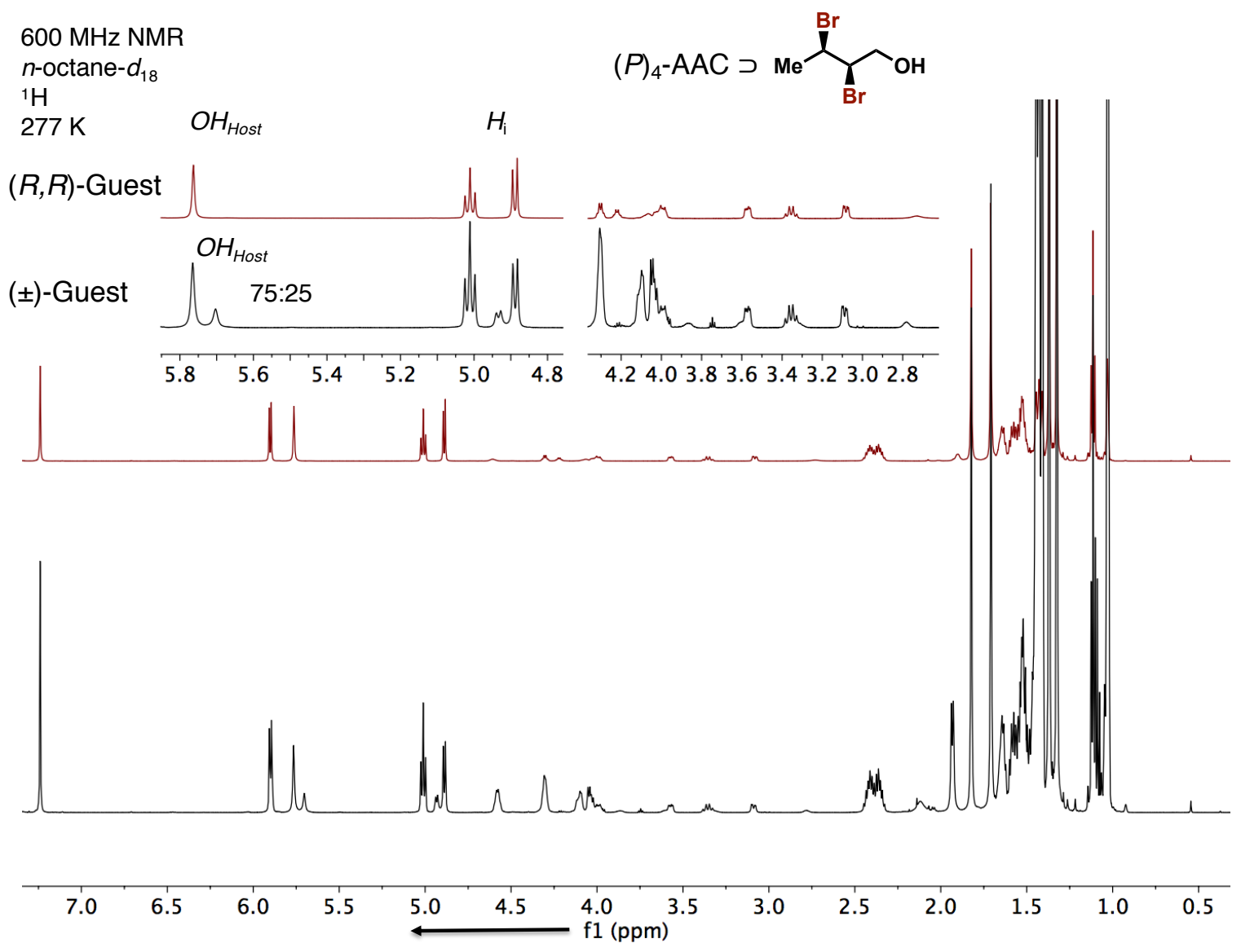

Figure S61. Overlay of the ${ }^{1} \mathrm{H}$ NMR $(600 \mathrm{MHz})$ traces of AAC $(P)_{4}-1$ in $\left[\mathrm{D}_{18}\right] n$-octane at $277 \mathrm{~K}$ with the enantiopure $(R, R)$-8 (top) and the racemic $\left(R^{*}, R^{*}\right)$-8 (bottom). Splitting of the OH-Host-resonances is observed only for the racemic $( \pm)$-guest $\left(R^{*}, R^{*}\right)-8$. 
$600 \mathrm{MHz}$ NMR

$n$-octane- $d_{18}$

${ }^{13} \mathrm{C}$

$277 \mathrm{~K}$

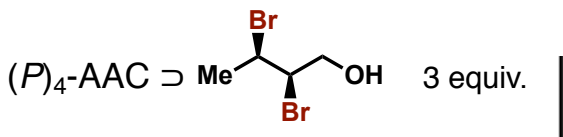

$( \pm)$

$\not \supset \mathrm{C}(3)-\mathrm{Br}_{\text {Guest }}$

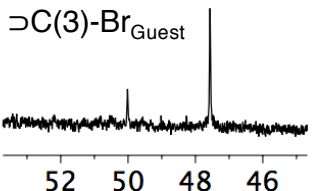

$\not \supset \mathrm{C}(3)-M e_{\text {Guest }}$

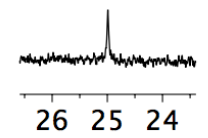

$\not \supset \mathrm{C}(2)-\mathrm{Br}_{\text {Guest }}$

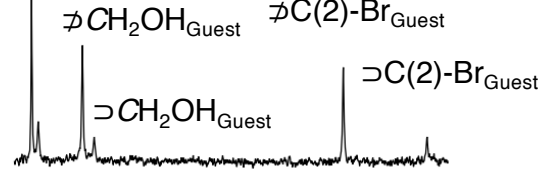

$\begin{array}{lllllll}65 & 64 & 63 & 62 & 61 & 60 & 59\end{array}$

$\begin{array}{lllllll}210.8 & 210.4 & 210.0 & 97 & 96 & 95\end{array}$

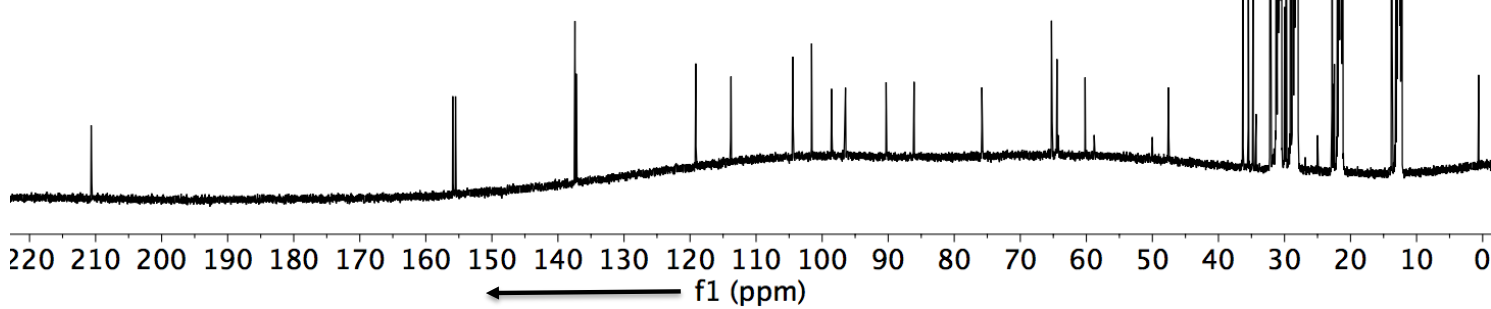

Figure S62. ${ }^{13} \mathrm{C}$ NMR $(150 \mathrm{MHz})$ traces of AAC $(P)_{4}-\mathbf{1}(7.0 \mathrm{mM})$ in $\left[\mathrm{D}_{18}\right] n$-octane at $277 \mathrm{~K}$ with 3 equiv. of $\left(R^{*}, R^{*}\right)-8$. The $\supset$ denotes the complexed guest and $D$ signifies the unbound at slow exchange on the NMRtimescale. 


\section{$600 \mathrm{MHz}$ NMR}

$n$-octane- $d_{18}$

${ }^{13} \mathrm{C}$

$277 \mathrm{~K}$

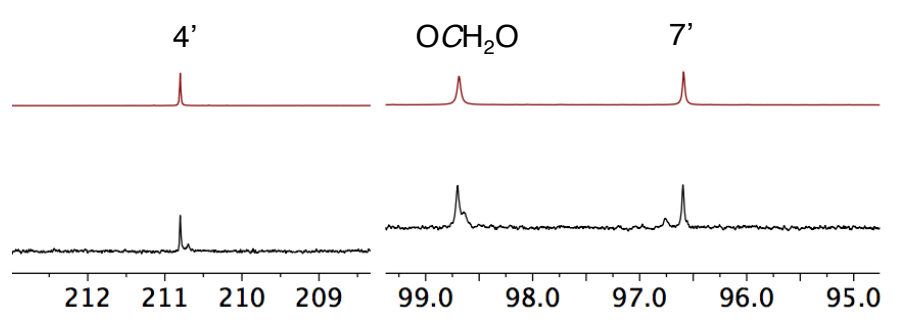

$(R, R)$-Guest

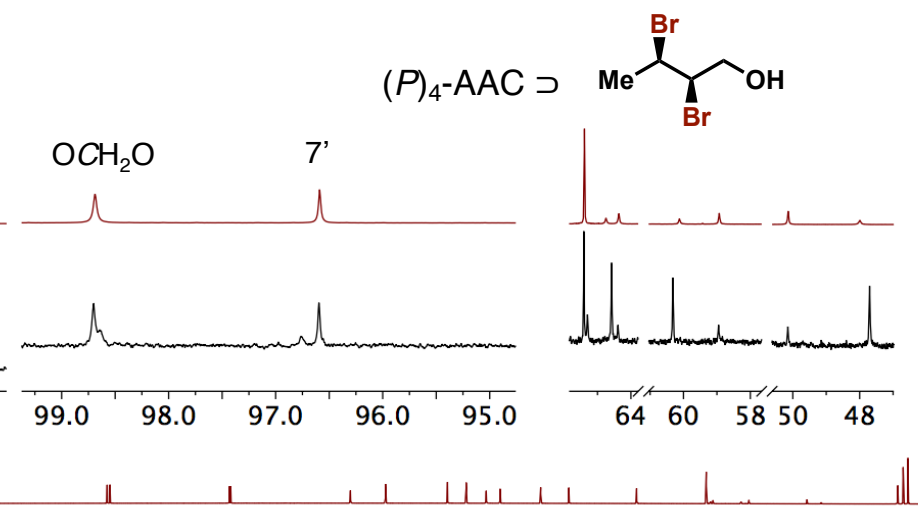

.

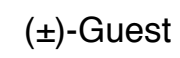

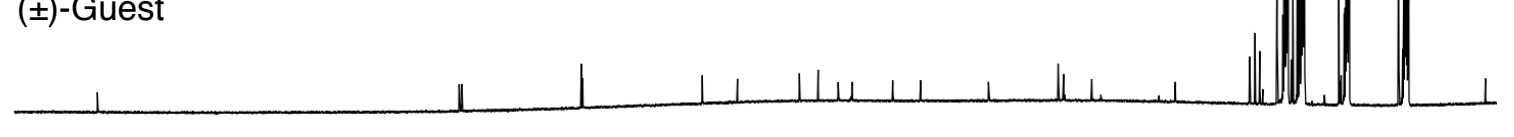

\begin{tabular}{lllllllllllllllllllllllllllll}
\hline 220 & 210 & 200 & 190 & 180 & 170 & 160 & 150 & 140 & 130 & 120 & 110 & 100 & 90 & 80 & 70 & 60 & 50 & 40 & 30 & 20 & 10 & 0
\end{tabular}

Figure S63. Overlay of the ${ }^{13} \mathrm{C}$ NMR $(150 \mathrm{MHz})$ traces of AAC $(P)_{4}-\mathbf{1}$ in $\left[\mathrm{D}_{18}\right] n$-octane at $277 \mathrm{~K}$ with the enantiopure $(R, R)-\mathbf{8}$ (top) and the racemic $\left(R^{*}, R^{*}\right)-\mathbf{8}$ (bottom). Splitting of the Host-resonances is observed only for the racemic $( \pm)$-guest $\left(R^{*}, R^{*}\right)-\mathbf{8}$. 
$600 \mathrm{MHz}$ NMR

$n$-octane- $d_{18}$

${ }^{1} \mathrm{H}$

$277 \mathrm{~K}$

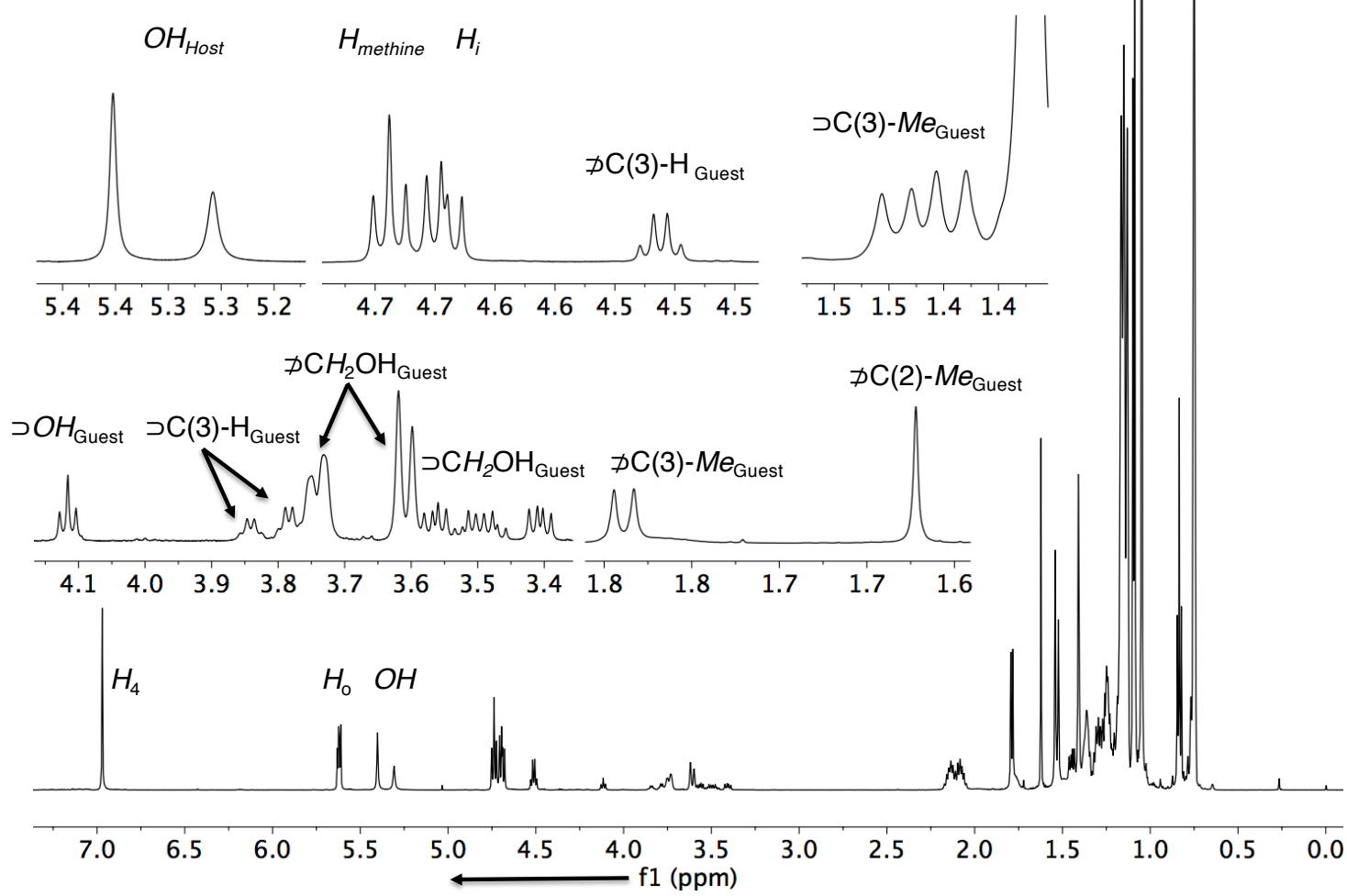

Figure S64. ${ }^{1} \mathrm{H}$ NMR $(600 \mathrm{MHz})$ traces of AAC $(P)_{4}-1(7.0 \mathrm{mM})$ in $\left[\mathrm{D}_{18}\right] n$-octane at $277 \mathrm{~K}$ with 3 equiv. of the $\left(R^{*}, S^{*}\right)-10$. The $\supset$ denotes the complexed guest and $D$ signifies the unbound at slow exchange on the NMRtimescale. Host-resonances: $\mathrm{H}_{\mathrm{i}}=$ inside protons and $\mathrm{H}_{\mathrm{o}}=$ outside protons of the methylene bridge; $\mathrm{H}_{4}=$ aromatic protons, highlighted in Figure S41. 


\section{$600 \mathrm{MHz}$ NMR}

$n$-octane- $d_{18}$

${ }^{13} \mathrm{C}$

$277 \mathrm{~K}$

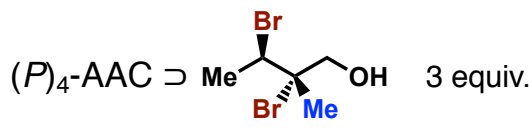

$( \pm)$

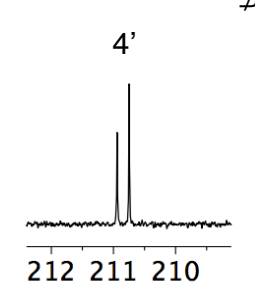

$\not \supset C(2)-B_{\text {Guest }}$
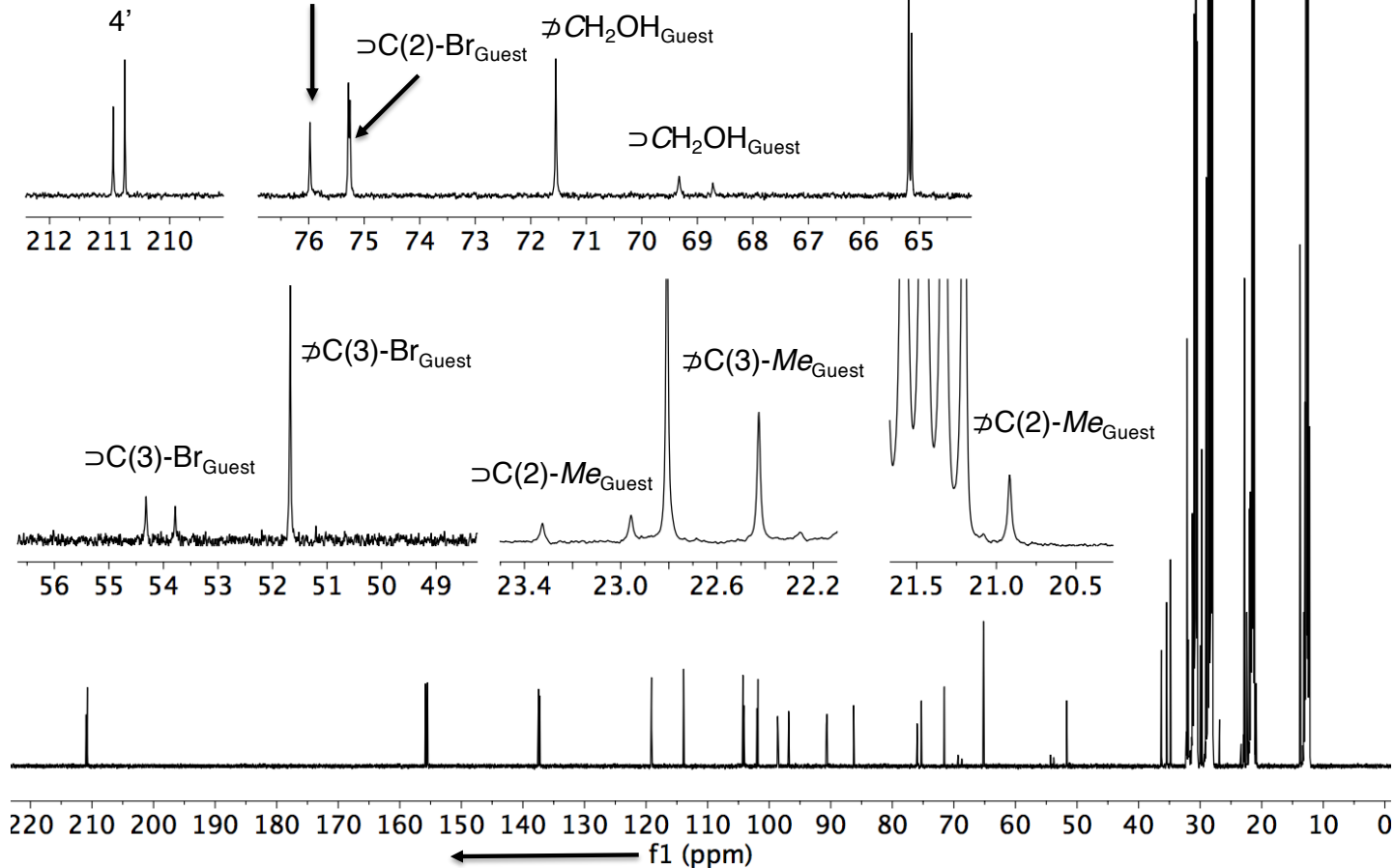

Figure S65. ${ }^{13} \mathrm{C}$ NMR $(150 \mathrm{MHz})$ traces of AAC $(P)_{4}-\mathbf{1}(7.0 \mathrm{mM})$ in $\left[\mathrm{D}_{18}\right] n$-octane at $277 \mathrm{~K}$ with 3 equiv. of $\left(R^{*}, S^{*}\right)$-10. The $\supset$ denotes the complexed guest and $D$ signifies the unbound at slow exchange on the NMRtimescale. 
$600 \mathrm{MHz}$ NMR

$n$-octane- $d_{18}$ ROESY

$277 \mathrm{~K}$
$(P)_{4}-\mathrm{AAC} \supset \mathrm{Me} \overbrace{\mathrm{Br}}^{\mathrm{Br}} \mathrm{OH} 3$ equiv.

$( \pm)$

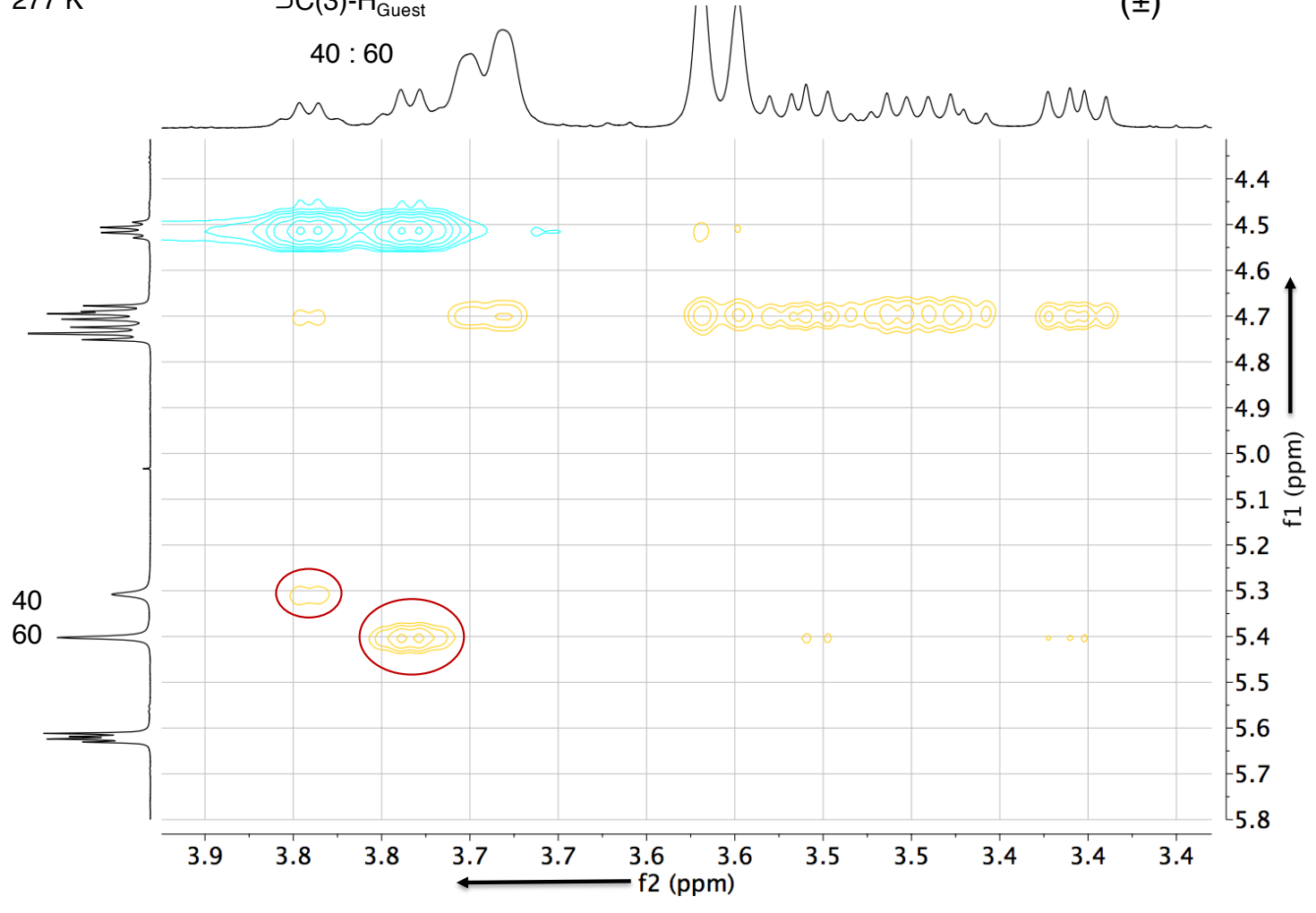

Figure S66. 2D ROESY NMR traces of AAC $(P)_{4}-1(7.0 \mathrm{mM})$ in $\left[\mathrm{D}_{18}\right] n$-octane at $277 \mathrm{~K}$ with 3 equiv. of $\left(R^{*}, S^{*}\right)$ 10. One equivalent of the guest is complexed to the interior of the host, while the second equivalent remains unbound in solution. The diastereoisomeric ratio of the host-guest complexes matches with the ratio of the splitting of the OH-host-resonance (60:40). 


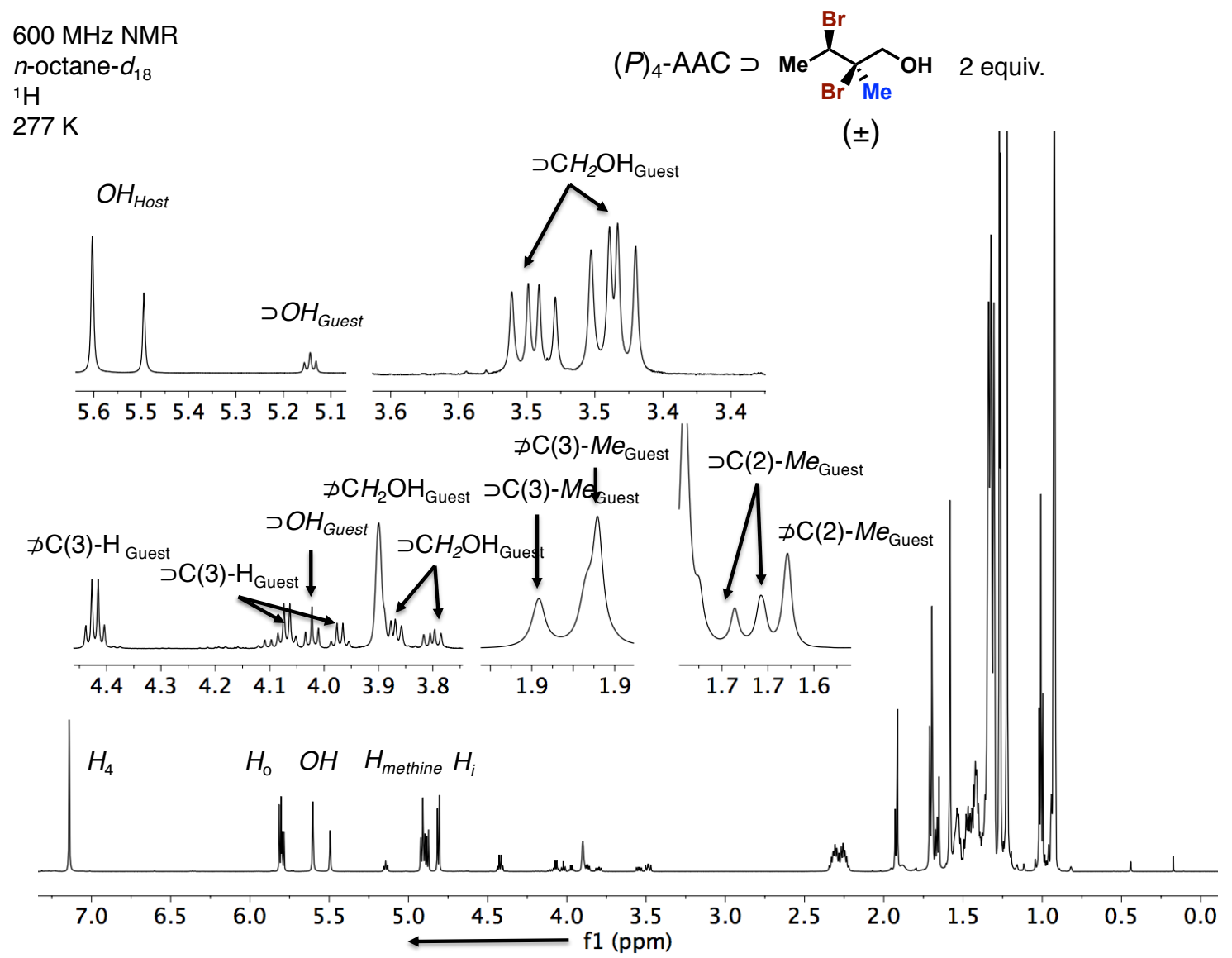

Figure S67. ${ }^{1} \mathrm{H}$ NMR $(600 \mathrm{MHz})$ traces of AAC $(P)_{4}-\mathbf{1}(7.0 \mathrm{mM})$ in $\left[\mathrm{D}_{18}\right] n$-octane at $277 \mathrm{~K}$ with 2 equiv. of the $\left(R^{*}, R^{*}\right)-10$. The $\supset$ denotes the complexed guest and $D$ signifies the unbound at slow exchange on the NMRtimescale. Host-resonances: $\mathrm{H}_{\mathrm{i}}=$ inside protons and $\mathrm{H}_{\mathrm{o}}=$ outside protons of the methylene bridge; $\mathrm{H}_{4}=$ aromatic protons, highlighted in Figure S41. 


\section{$600 \mathrm{MHz}$ NMR}

$n$-octane- $d_{18}$

${ }^{13} \mathrm{C}$

$277 \mathrm{~K}$

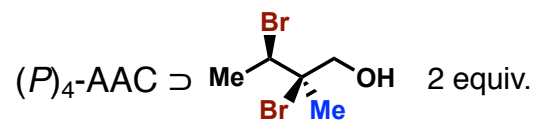

$( \pm)$

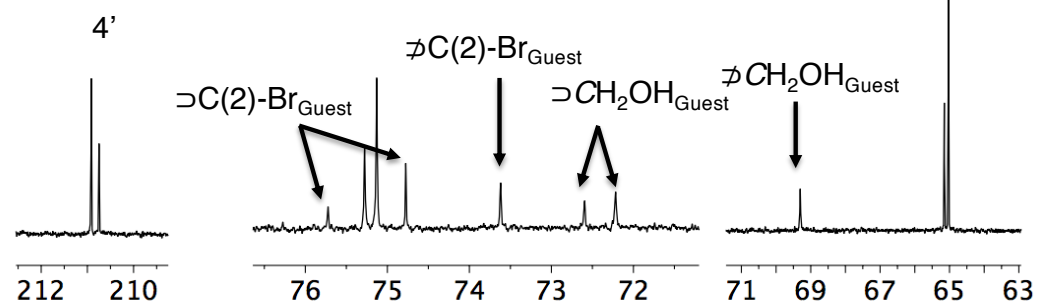

$\not \supset \mathrm{C}(3)-\mathrm{Br}_{\text {Guest }}$

$\not \supset \mathrm{C}(2)-M e_{\text {Guest }}$

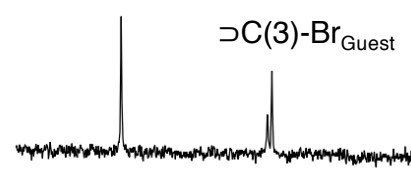

$\not \supset \mathrm{C}(3)-M e_{\text {Guest }}$
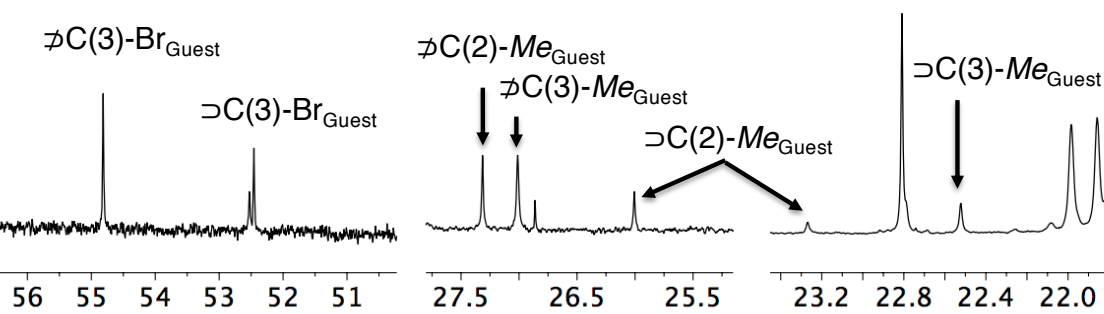

$27.5 \quad 26.5 \quad 25.5$

$\begin{array}{lllll}23.2 & 22.8 & 22.4 & 22.0\end{array}$

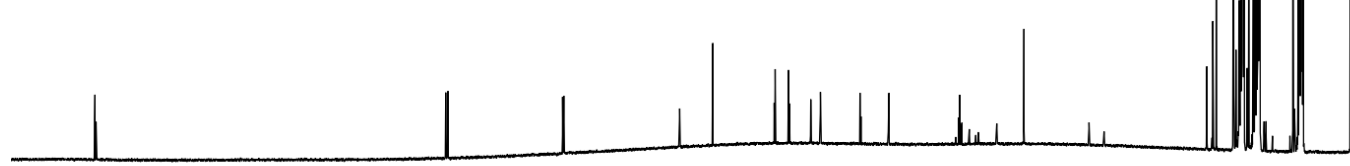

$\begin{array}{lllllllllllllllllllllll}220 & 210 & 200 & 190 & 180 & 170 & 160 & 150 & 140 & 130 & 120 & 110 & 100 & 90 & 80 & 70 & 60 & 50 & 40 & 30 & 20 & 10\end{array}$ $\mathrm{f} 1(\mathrm{ppm})$

Figure S68. ${ }^{13} \mathrm{C}$ NMR $(150 \mathrm{MHz})$ traces of AAC $(P)_{4}-1(7.0 \mathrm{mM})$ in $\left[\mathrm{D}_{18}\right] n$-octane at $277 \mathrm{~K}$ with 3 equiv. of $\left(R^{*}, R^{*}\right)$-10. The $\supset$ denotes the complexed guest and $D$ signifies the unbound at slow exchange on the NMRtimescale. 


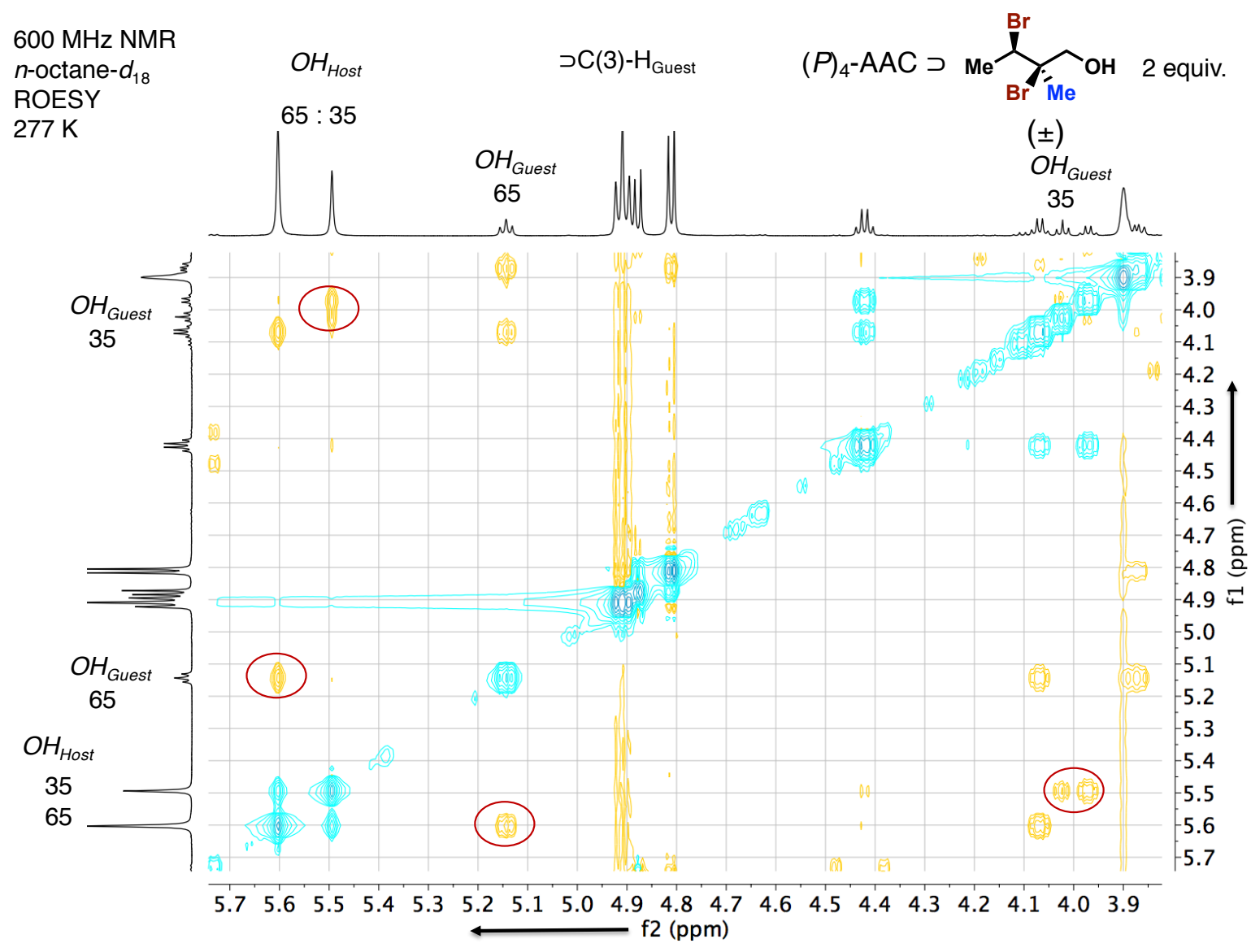

Figure S69. 2D ROESY NMR traces of AAC $(P)_{4}-1(7.0 \mathrm{mM})$ in $\left[\mathrm{D}_{18}\right] n$-octane at $277 \mathrm{~K}$ with 3 equiv. of $\left(R^{*}, R^{*}\right)$ 10. One equivalent of the guest is complexed to the interior of the host, while the second equivalent remains unbound in solution. The diastereoisomeric ratio of the host-guest complexes matches with the ratio of the splitting of the OH-host-resonance (65:35). 
$600 \mathrm{MHz}$ NMR

$n$-octane- $d_{18}$

${ }^{1} \mathrm{H}$

$277 \mathrm{~K}$
$(P)_{4}-\mathrm{AAC} \supset \mathrm{F}_{3} \mathrm{C} \underbrace{\mathrm{Br}}_{\overline{\mathrm{B} r}} \mathrm{OH} 4$ equiv.

$( \pm)$

$\pm)$
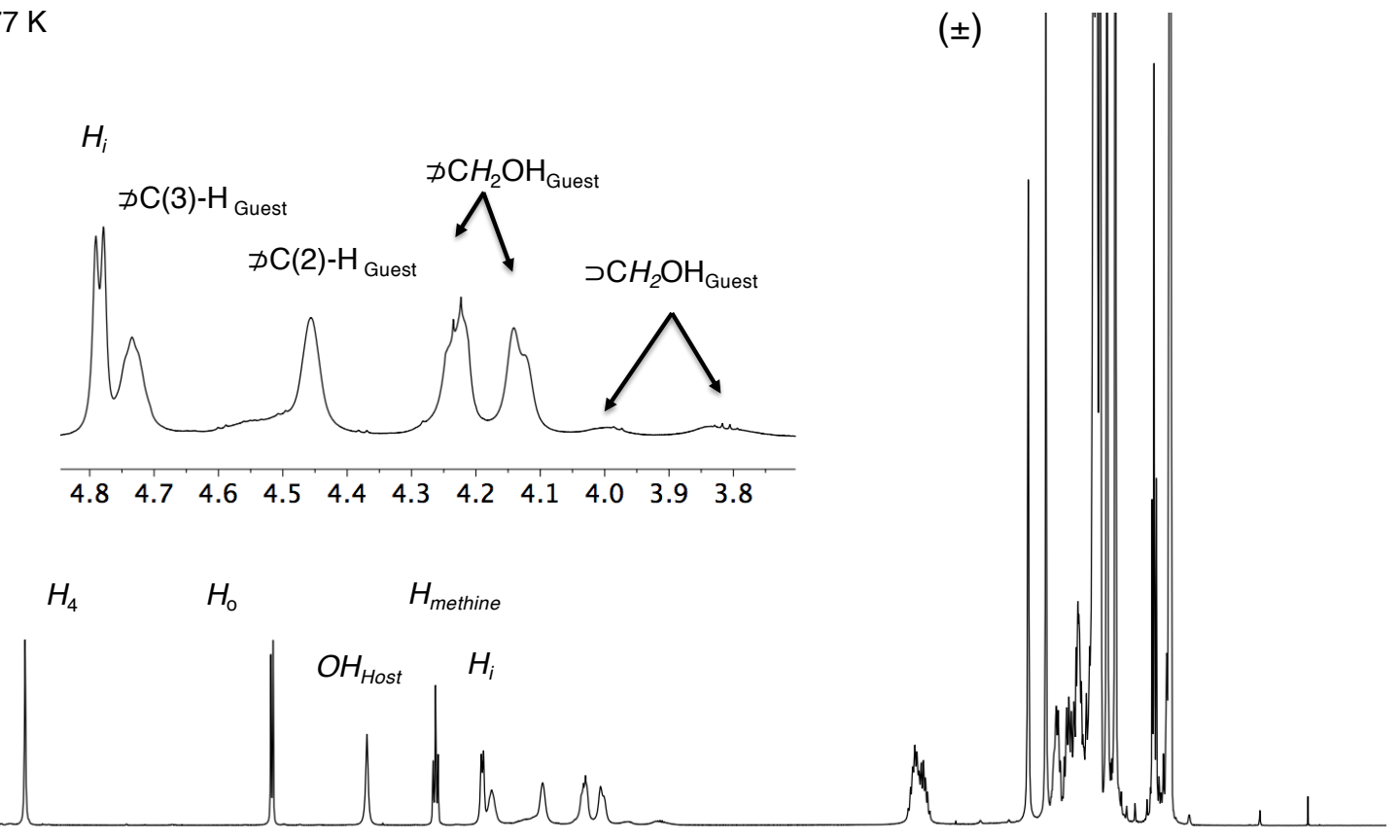

$3.0 \quad 2.5$

f1 (ppm)

Figure S70. ${ }^{1} \mathrm{H}$ NMR $(600 \mathrm{MHz})$ traces of AAC $(P)_{4-1}(7.0 \mathrm{mM})$ in $\left[\mathrm{D}_{18}\right] n$-octane at $277 \mathrm{~K}$ with 4 equiv. of the $\left(R^{*}, S^{*}\right)$-11. The $\supset$ denotes the complexed guest and $D$ signifies the unbound at slow exchange on the NMRtimescale. Host-resonances: $\mathrm{H}_{\mathrm{i}}=$ inside protons and $\mathrm{H}_{\mathrm{o}}=$ outside protons of the methylene bridge; $\mathrm{H}_{4}=$ aromatic protons, highlighted in Figure S41. 
$600 \mathrm{MHz}$ NMR

${ }^{13} \mathrm{C}$

$277 \mathrm{~K}$

$n$-octane- $d_{18}$

$$
(P)_{4} \text {-AAC } \supset \mathrm{F}_{3} \mathrm{C} \underbrace{\mathrm{Br}}_{\overline{\mathrm{B} r}} \mathrm{OH}
$$

$( \pm)$
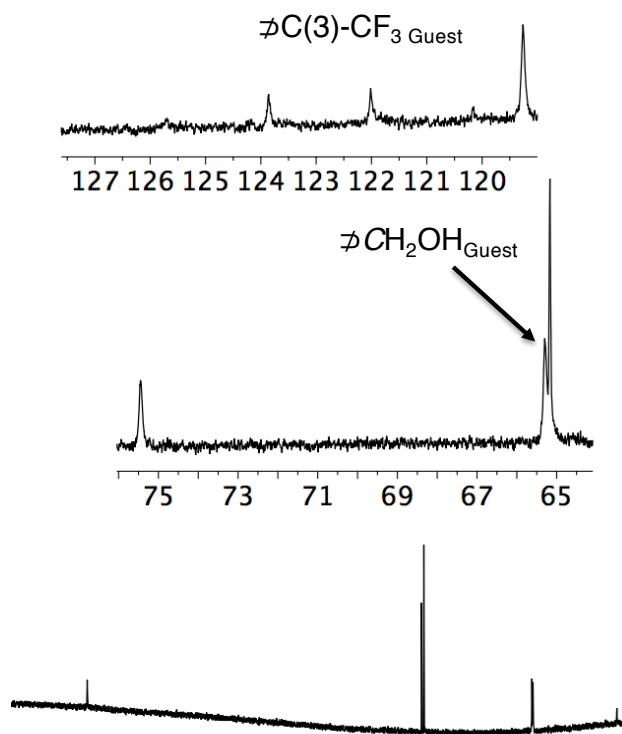

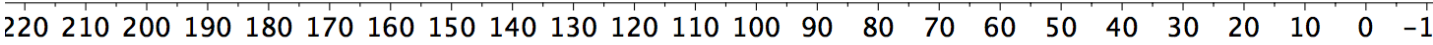

$\not \supset \mathrm{C}(2)-\mathrm{Br}_{\text {Guest }}$

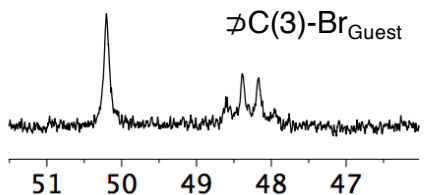

Figure S71. ${ }^{13} \mathrm{C}$ NMR $(150 \mathrm{MHz})$ traces of AAC $(P)_{4}-1(7.0 \mathrm{mM})$ in $\left[\mathrm{D}_{18}\right] n$-octane at $277 \mathrm{~K}$ with 4 equiv. of $\left(R^{*}, S^{*}\right)-11$. The $D$ signifies the unbound guest. 
$600 \mathrm{MHz}$ NMR

$n$-octane- $d_{18}$

${ }^{19} \mathrm{~F}$

$277 \mathrm{~K}$

$(P)_{4}$-AAC $\supset \mathrm{F}_{3} \mathrm{C} \overbrace{\dot{\bar{B} \mathrm{Br}}}^{\mathrm{Br}} \mathrm{OH} 4$ equiv.

$( \pm)$

$238 \mathrm{~K}$

$\supset \mathrm{C}(3)-\mathrm{CF}_{3 \mathrm{Guest}}$

$40: 60$

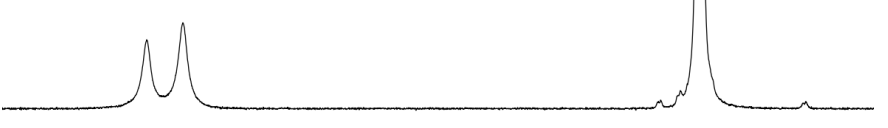

$-65.0-65.4-65.8-66.2-66.6-67.0-67.4-67.8-68.2$

$\supset \mathrm{C}(3)-\mathrm{CF}_{3 \mathrm{Guest}}$

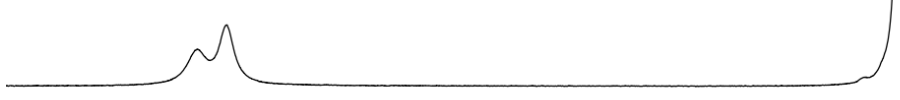

$\not \supset \mathrm{C}(3)-\mathrm{CF}_{3 \mathrm{Guest}}$

\begin{tabular}{llllllllllll}
\hline-64.0 & -64.5 & -65.0 & -65.5 & -66.0 & -66.5 & -67.0 & -67.5 & -68.0 & -68.5 & -69.0 & -69.5 \\
& & & & $f(\mathrm{ppm})$ & & & &
\end{tabular}

Figure S72. ${ }^{19} \mathrm{~F}$ NMR traces of AAC $(P)_{4}-\mathbf{1}(7.0 \mathrm{mM})$ in $\left[\mathrm{D}_{18}\right] n$-octane at $277 \mathrm{~K}$ with 4 equiv. of $\left(R^{*}, S^{*}\right)-\mathbf{1 1}$. The $\supset$ denotes the complexed guest and $D$ signifies the unbound at slow exchange on the NMR-timescale. VT- ${ }^{1} \mathrm{H}$ NMR traces of $(P)_{4}$-AAC $(7.0 \mathrm{mM})$ in [ $\left.\mathrm{D}_{18}\right] n$-octane at $238 \mathrm{~K}$ with 3 equiv. of guest are added to the spectra. 
$600 \mathrm{MHz} N M R$
$n$-octane- $d_{18}$
${ }^{1} \mathrm{H}$
$277 \mathrm{~K}$
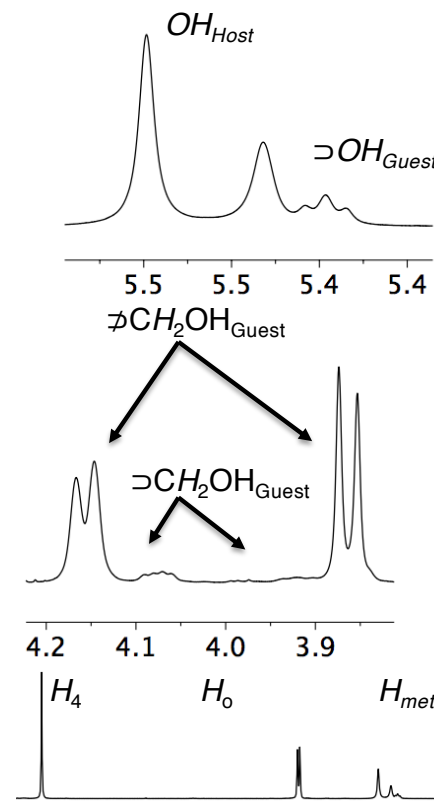

$\begin{array}{llllllll}2.6 & 2.5 & 2.5 & 2.4 & 2.4 & 2.3 & 2.3\end{array}$

$H_{\text {methine }} H_{i}$

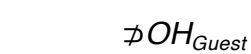

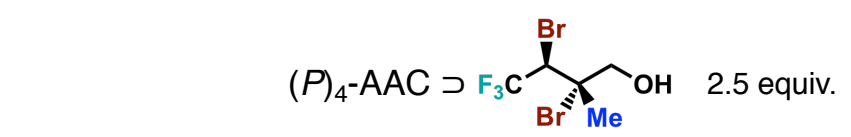

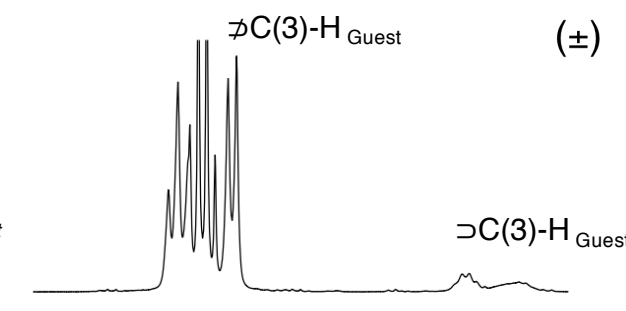

$( \pm)$

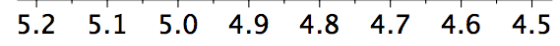

$\not \supset \mathrm{C}(2)-M e_{\text {Guest }}$

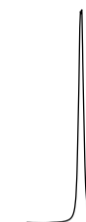

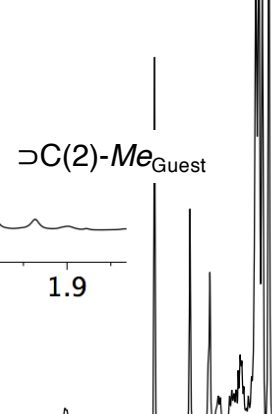

$M$
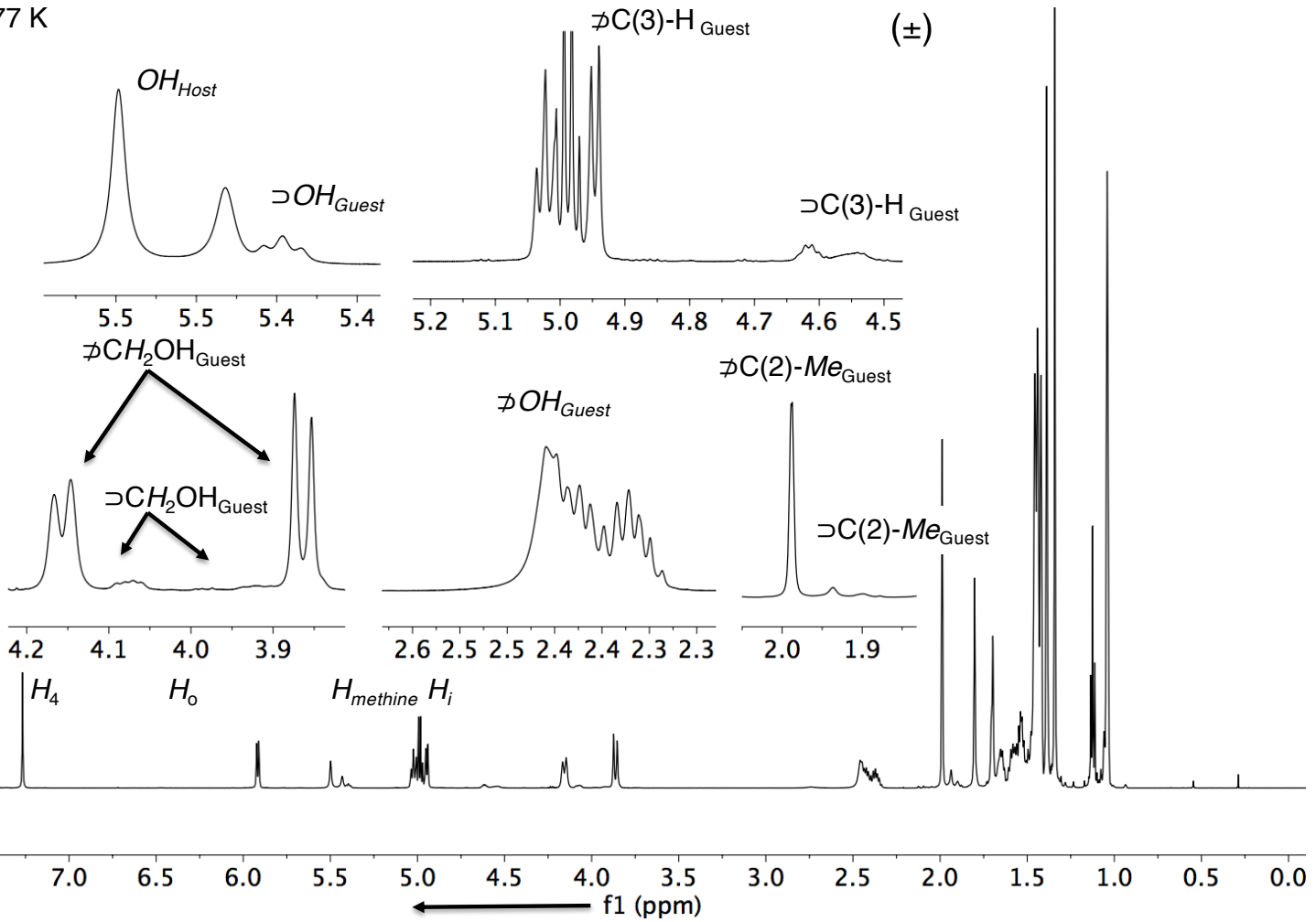

Figure S73. ${ }^{1} \mathrm{H}$ NMR $(600 \mathrm{MHz})$ traces of AAC $(P)_{4}-1(7.0 \mathrm{mM})$ in $\left[\mathrm{D}_{18}\right] n$-octane at $277 \mathrm{~K}$ with 2.5 equiv. of the $\left(R^{*}, S^{*}\right)$-12. The $\supset$ denotes the complexed guest and $D$ signifies the unbound at slow exchange on the NMRtimescale. Host-resonances: $\mathrm{H}_{\mathrm{i}}=$ inside protons and $\mathrm{H}_{\mathrm{o}}=$ outside protons of the methylene bridge; $\mathrm{H}_{4}=$ aromatic protons, highlighted in Figure S41. 


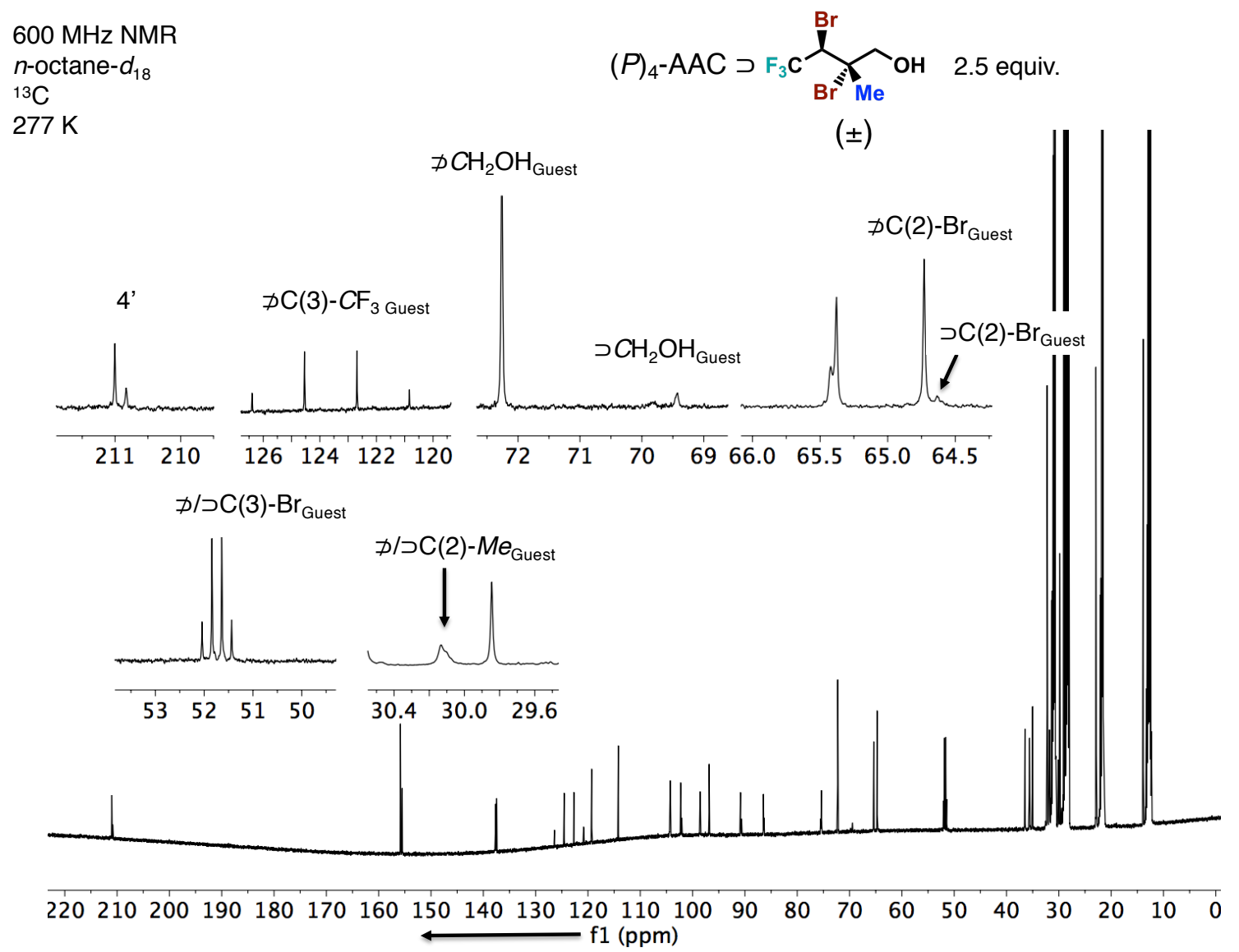

Figure S74. ${ }^{13} \mathrm{C}$ NMR $(150 \mathrm{MHz})$ traces of AAC $(P)_{4}-\mathbf{1}(7.0 \mathrm{mM})$ in $\left[\mathrm{D}_{18}\right] n$-octane at $277 \mathrm{~K}$ with 2.5 equiv. of $\left(R^{*}, S^{*}\right)$-12. The $\supset$ denotes the complexed guest and $D$ signifies the unbound at slow exchange on the NMRtimescale. 
$600 \mathrm{MHz}$ NMR

$n$-octane- $d_{18}$

${ }^{19} \mathrm{~F}$

$277 \mathrm{~K}$

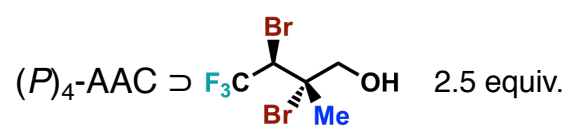

$( \pm)$

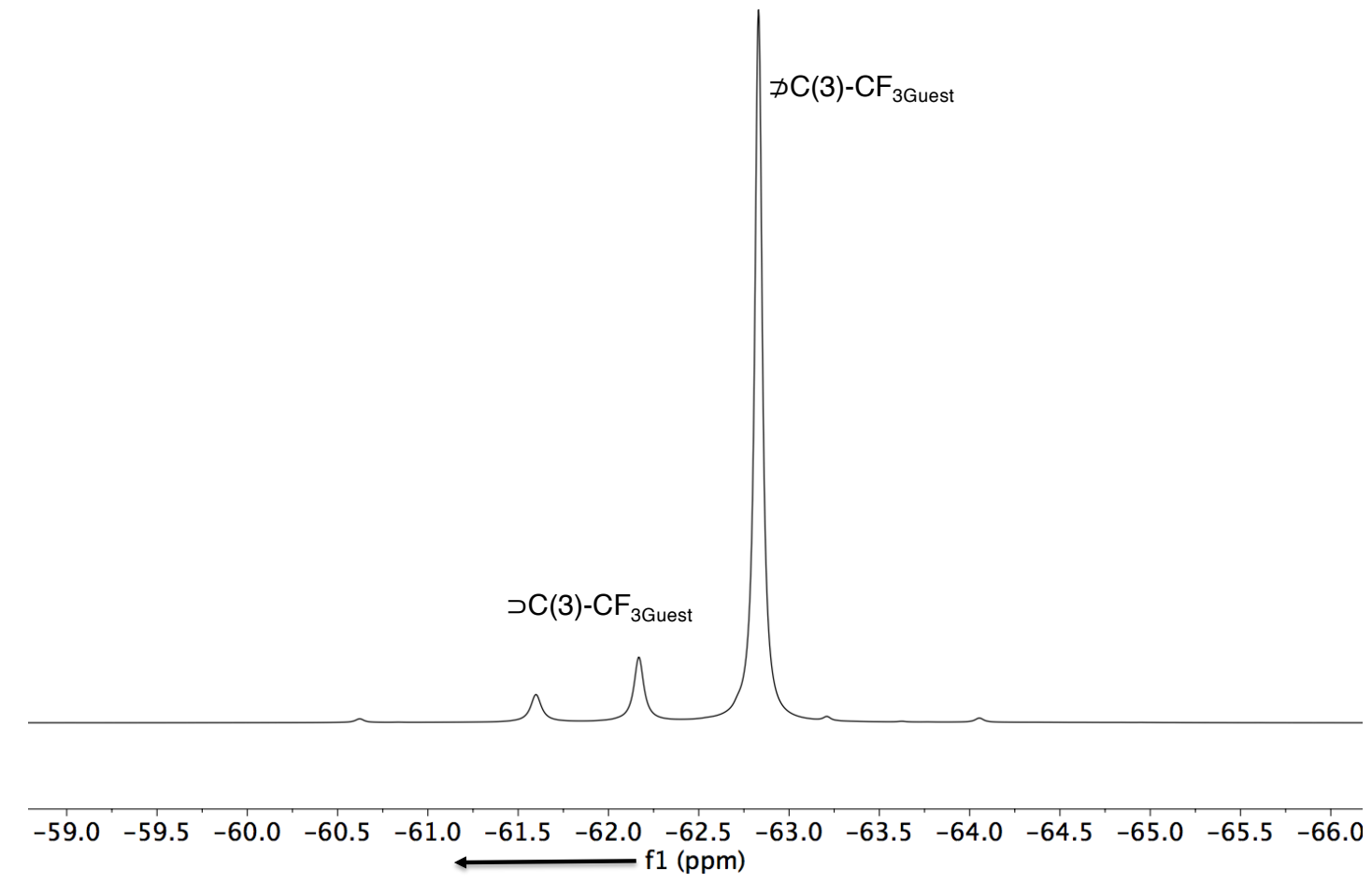

Figure S75. ${ }^{19} \mathrm{~F}$ NMR traces of AAC $(P)_{4}-\mathbf{1}(7.0 \mathrm{mM})$ in $\left[\mathrm{D}_{18}\right] n$-octane at $277 \mathrm{~K}$ with 2.5 equiv. of $\left(R^{*}, S^{*}\right)-\mathbf{1 2}$. The $\supset$ denotes the complexed guest and $D$ signifies the unbound at slow exchange on the NMR-timescale. 


\section{S9. Splitting of the Host-OH-Bonding Array upon Complexation of Guests}

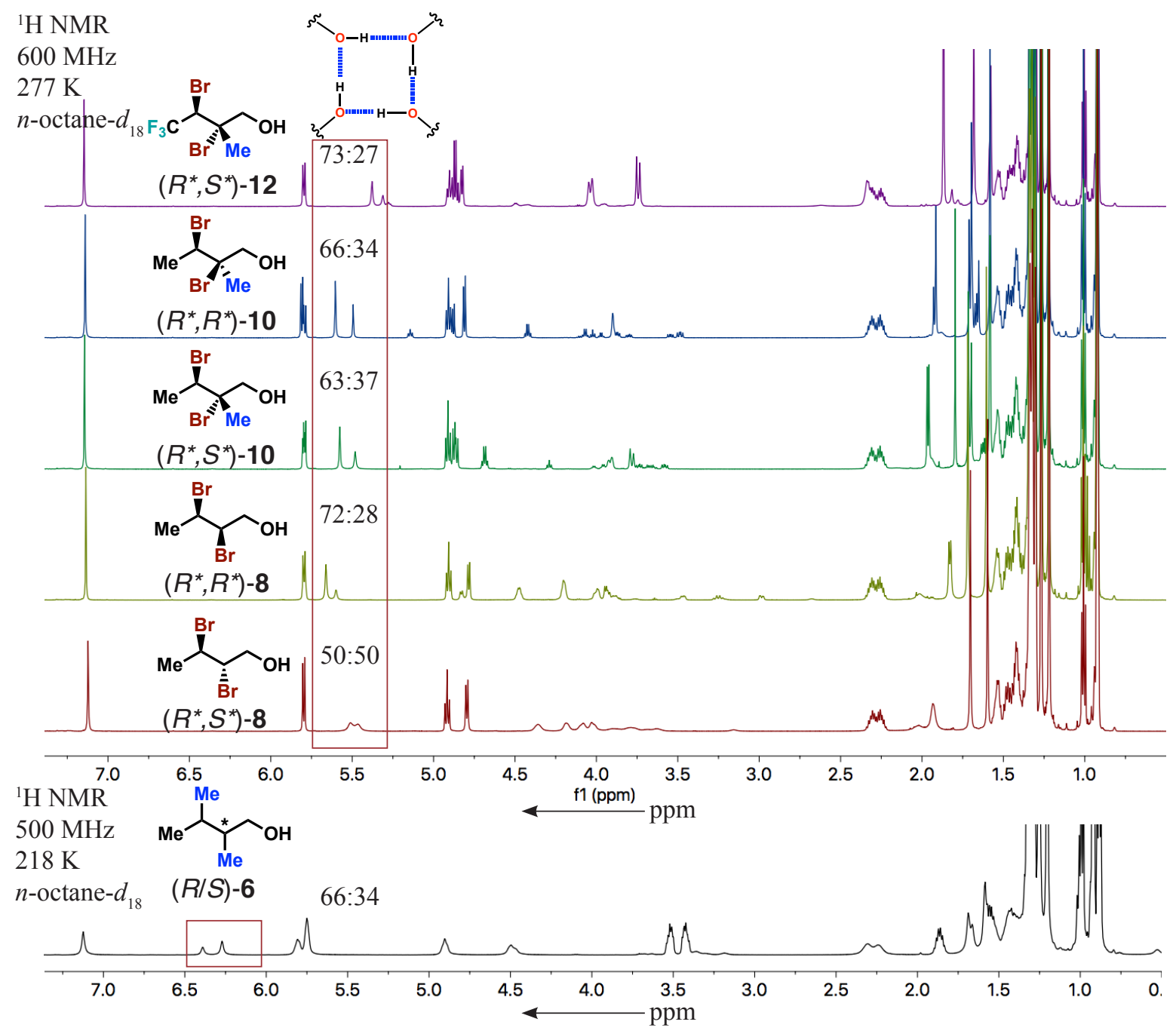

Figure S76. Splitting of the host-OH-bonding array of AAC $(P)_{4}-1$ upon complexation of the racemic guests, as observed in the ${ }^{1} \mathrm{H}$ NMR spectroscopic traces. ${ }^{[8]}$

\section{S10. J-Based Conformational Analysis of Guests 7 and 8 in Acetonitrile}

Homo- and hetero-nuclear coupling constants were extracted from ${ }^{1} \mathrm{H}-\mathrm{NMR}$ and HSQCHECADE spectra. ${ }^{[9]}$ Individual peak assignments are based on ${ }^{1} \mathrm{H},{ }^{13} \mathrm{C}, \mathrm{HSQC}, \mathrm{HMBC}$ and COSY spectra.

A summary of the conformational analysis of alcohols $\left(R^{*}, S^{*}\right)-,\left(R^{*}, R^{*}\right)-7$ and $\left(R^{*}, S^{*}\right)-$, $\left(R^{*}, R^{*}\right)-\mathbf{8}$ is given in Figure $\mathrm{S} 77$. 
<smiles>C[C@H](Cl)[C@H](Cl)CO</smiles>
$\left(R^{\star}, S^{\star}\right)-7$
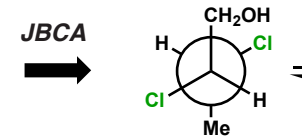
major conformer $\mathrm{CH}_{3} \mathrm{CN}$<smiles>C[C@H](Cl)[C@H](Cl)CO</smiles>

$\left(R^{\star}, R^{\star}\right)-7$

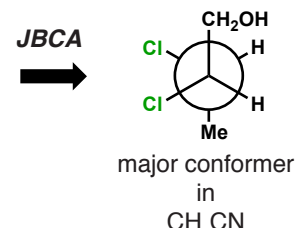

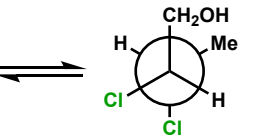

minor conformer in $\mathrm{CH}_{3} \mathrm{CN}$

B

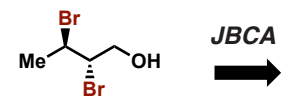

$\left(R^{\star}, S^{\star}\right)-8$

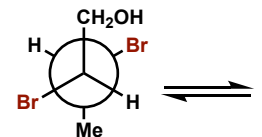

major conformer in

$\mathrm{CH}_{3} \mathrm{CN}$

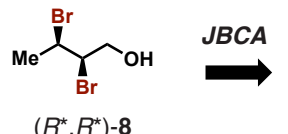

$\left(R^{\star}, R^{\star}\right)-8$

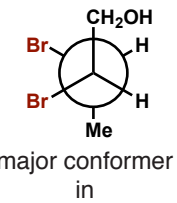

$\mathrm{CH}_{3} \mathrm{CN}$

Figure S77. Conformational analysis of guests $\left(R^{*}, S^{*}\right)$ - and $\left(R^{*}, R^{*}\right)-7$, and $\left(R^{*}, S^{*}\right)$ - and $\left(R^{*}, R^{*}\right)-\mathbf{8}$ in $\mathrm{CD}_{3} \mathrm{CN}$ at $298 \mathrm{~K}$ through 1 and 2D NMR spectroscopic experiments.

\section{$600 \mathrm{MHz}$ NMR}

acetonitrile- $d_{3}$

${ }^{1} \mathrm{H}$

$298 \mathrm{~K}$

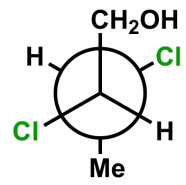<smiles>C[C@H](Cl)[C@H](Cl)CO</smiles>

(士)
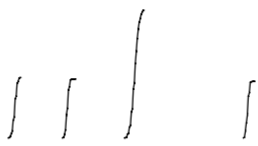

Me

\begin{tabular}{|c|c|c|c|c|c|}
\hline${ }^{3} J(\mathrm{H} 2, \mathrm{H} 3)$ & $+5.8 \mathrm{~Hz}$ & large & & & \\
\hline $\begin{array}{l}{ }^{3} J(\mathrm{H} 2, \mathrm{C} 4) \\
{ }^{3} J(\mathrm{H} 3, \mathrm{C} 1)\end{array}$ & $\begin{array}{l}+3.6 \mathrm{~Hz} \\
+2.5 \mathrm{~Hz}\end{array}$ & $\begin{array}{l}\text { small } \\
\text { small }\end{array}$ & & & \\
\hline${ }^{2} J(\mathrm{H} 2, \mathrm{C} 3)$ & $-3.5 \mathrm{~Hz}$ & small & $\begin{array}{c}\text { A (qd) } \\
4.41\end{array}$ & $\begin{array}{l}C(\mathrm{~m}) \\
3.80\end{array}$ & \begin{tabular}{|l}
$E(t)$ \\
3.23 \\
\end{tabular} \\
\hline${ }^{2} J(\mathrm{H} 3, \mathrm{C} 2)$ & $-1.5 \mathrm{~Hz}$ & small & & & \\
\hline
\end{tabular}

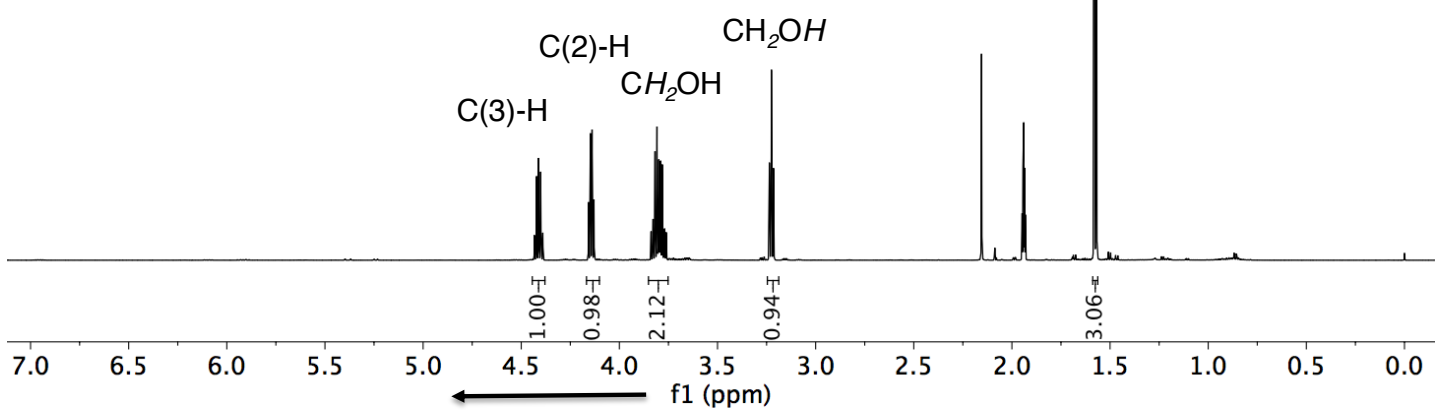

Figure S78. ${ }^{1} \mathrm{H}$ NMR $(600 \mathrm{MHz})$ traces of $\left(R^{*}, S^{*}\right)-7$ in acetonitrile- $d_{3}$ at $298 \mathrm{~K}$. $J$-based coupling constants are assigned and given in the table. 
$150 \mathrm{MHz}$ NMR

acetonitrile- $d_{3}$

${ }^{13} \mathrm{C}$

$298 \mathrm{~K}$<smiles>C[C@H](Cl)[C@H](Cl)CO</smiles>

$( \pm)$

$\begin{array}{lll}m & n & \\ 0 & m & 0 \\ 0 & m & 0 \\ 0 & 0 & 0 \\ 1 & 1 & 1\end{array}$

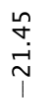

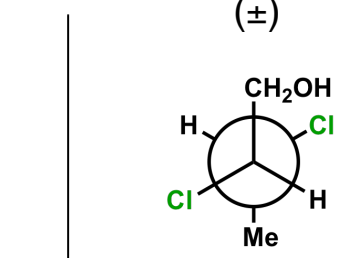

$\mathrm{CH}_{2} \mathrm{OH} \quad \mathrm{Me}$
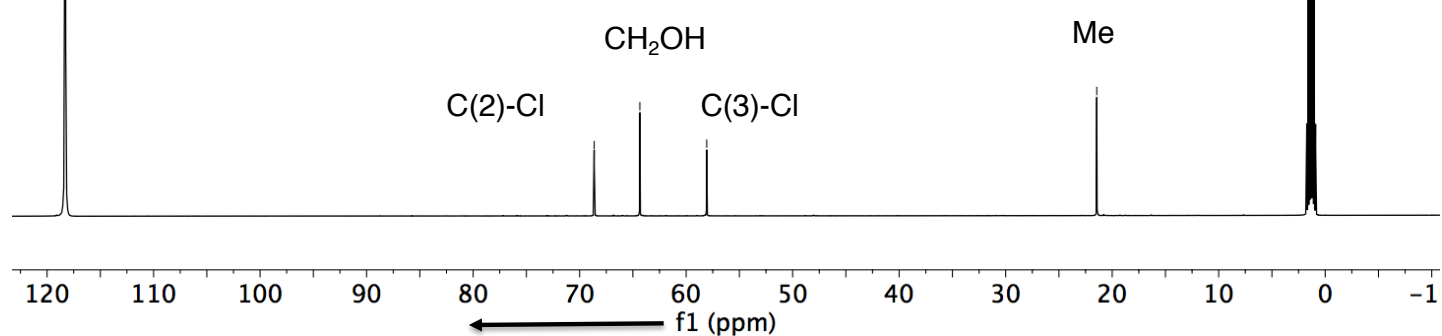

Figure S79. ${ }^{13} \mathrm{C}$ NMR $(150 \mathrm{MHz})$ traces of $\left(R^{*}, S^{*}\right)-7$ in acetonitrile- $d_{3}$ at $298 \mathrm{~K}$.

\section{$600 \mathrm{MHz}$ NMR \\ acetonitrile- $d_{3}$ \\ ${ }^{1} \mathrm{H}$}

$298 \mathrm{~K}$

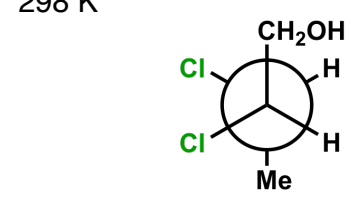<smiles>C[C@H](Cl)[C@H](Cl)CO</smiles>

$( \pm)$

\begin{tabular}{|c|c|c|c|c|c|c|}
\hline${ }^{3} \mathrm{~J}(\mathrm{H} 2, \mathrm{H} 3)$ & $+2.7 \mathrm{~Hz}$ & small & & & & I \\
\hline${ }^{3} J(\mathrm{H} 2, \mathrm{C} 4)$ & $+2.2 \mathrm{~Hz}$ & small & & & & \\
\hline${ }^{3} J(\mathrm{H} 3, \mathrm{C} 1)$ & $+1.8 \mathrm{~Hz}$ & small & & & & \\
\hline${ }^{2} J(\mathrm{H} 2, \mathrm{C} 3)$ & $+1.8 \mathrm{~Hz}$ & small & $\begin{array}{c}\text { A (qd) } \\
4.54 \\
\end{array}$ & \begin{tabular}{|c}
$B(t d)$ \\
4.10 \\
\end{tabular} & $\begin{array}{l}E(m) \\
3.74 \\
\end{array}$ & $\begin{array}{l}C(t) \\
3.28 \\
\end{array}$ \\
\hline${ }^{2} J(\mathrm{H} 3, \mathrm{C} 2)$ & $+1.2 \mathrm{~Hz}$ & small & & & & \\
\hline
\end{tabular}

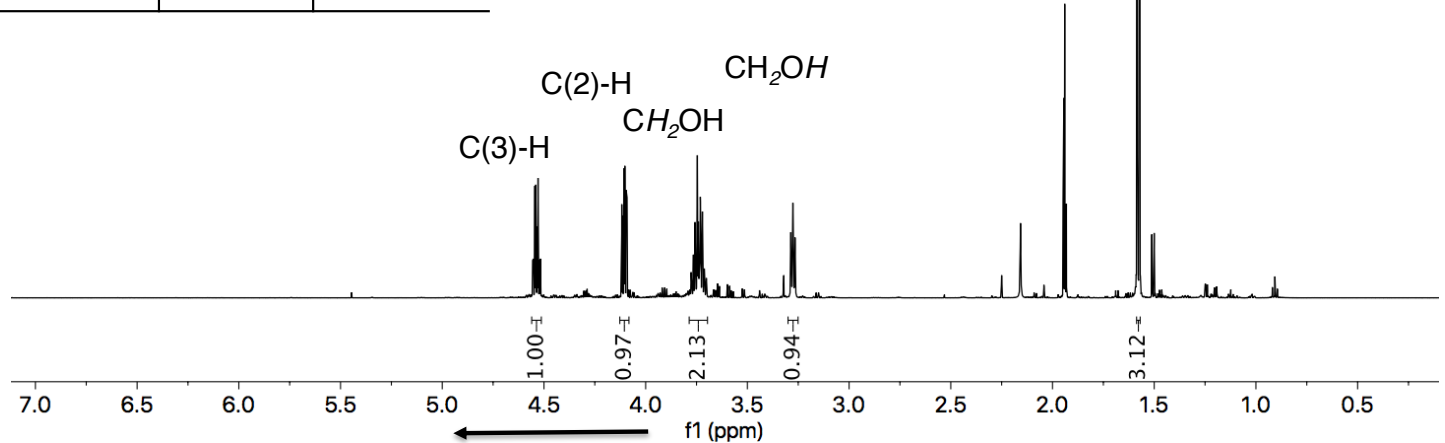

Figure S80. ${ }^{1} \mathrm{H}$ NMR $(600 \mathrm{MHz})$ traces of $\left(R^{*}, R^{*}\right)-7$ in acetonitrile- $d_{3}$ at $298 \mathrm{~K} . J$-based coupling constants are assigned and given in the table. 
<smiles>C[C@H](Cl)[C@H](Cl)CO</smiles>

$( \pm)$

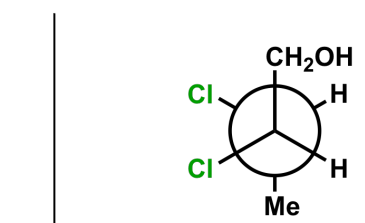

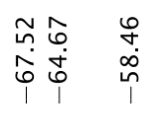
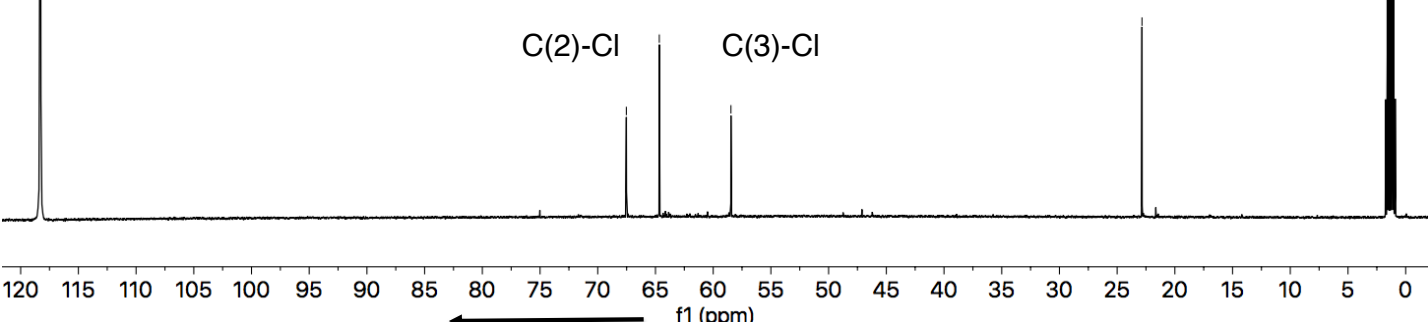

Figure S81. ${ }^{13} \mathrm{C}$ NMR $(150 \mathrm{MHz})$ traces of $\left(R^{*}, R^{*}\right)-7$ in acetonitrile- $d_{3}$ at $298 \mathrm{~K}$.

$600 \mathrm{MHz}$ NMR

acetonitrile- $d_{3}$

${ }^{1} \mathrm{H}$

$298 \mathrm{~K}$<smiles>CC1C(CO)C2CC3(Br)CC1C2C(Br)C3Br</smiles>

\begin{tabular}{l|l|l}
\hline${ }^{3} \mathrm{~J}(\mathrm{H} 2, \mathrm{H} 3)$ & $+6.9 \mathrm{~Hz}$ & large \\
\hline${ }^{3} \mathrm{~J}(\mathrm{H} 2, \mathrm{C} 4)$ & $+3.3 \mathrm{~Hz}$ & small \\
${ }^{3} \mathrm{~J}(\mathrm{H} 3, \mathrm{C} 1)$ & $+2.3 \mathrm{~Hz}$ & small \\
\hline${ }^{2} \mathrm{~J}(\mathrm{H} 2, \mathrm{C} 3)$ & $-3.5 \mathrm{~Hz}$ & small \\
${ }^{2} \mathrm{~J}(\mathrm{H} 3, \mathrm{C} 2)$ & $-1.5 \mathrm{~Hz}$ & small \\
\hline
\end{tabular}
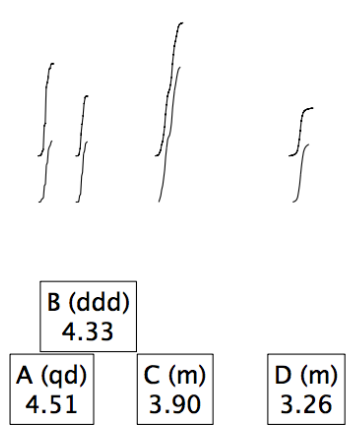<smiles>C[C](Br)[C](Br)CO</smiles>

$( \pm)$

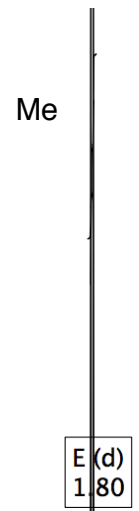

$\mathrm{C}(2)-\mathrm{H}$

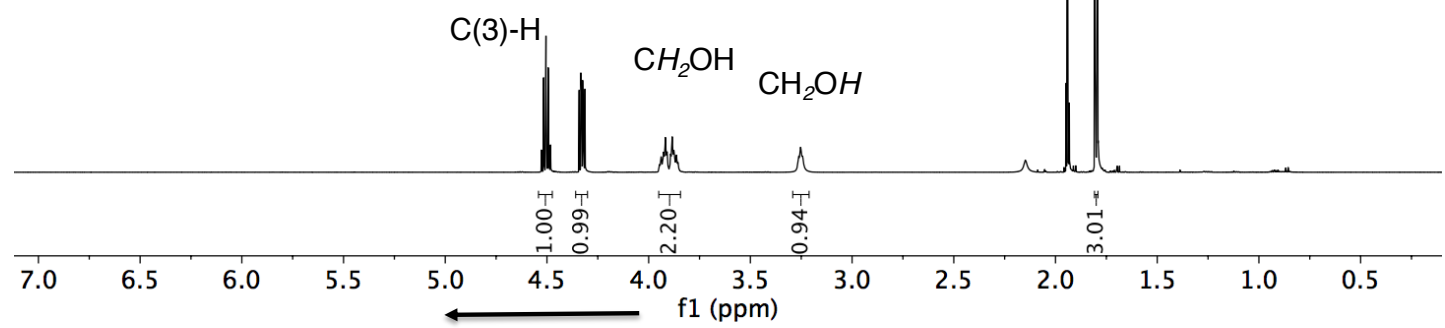

Figure S82. ${ }^{1} \mathrm{H}$ NMR $(600 \mathrm{MHz})$ traces of $\left(R^{*}, S^{*}\right)-8$ in acetonitrile- $d_{3}$ at $298 \mathrm{~K} . J$-based coupling constants are assigned and given in the table. 
<smiles>C[C@@H](Br)[C@H](Br)CO</smiles>

$( \pm)$

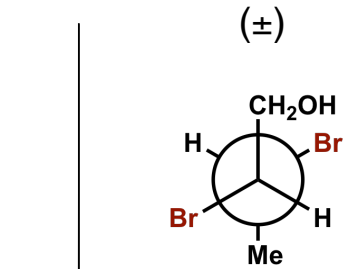

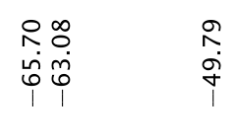

$\stackrel{\substack{n \\ \stackrel{1}{\sim}}}{\text { 1 }}$

\footnotetext{
Me
}
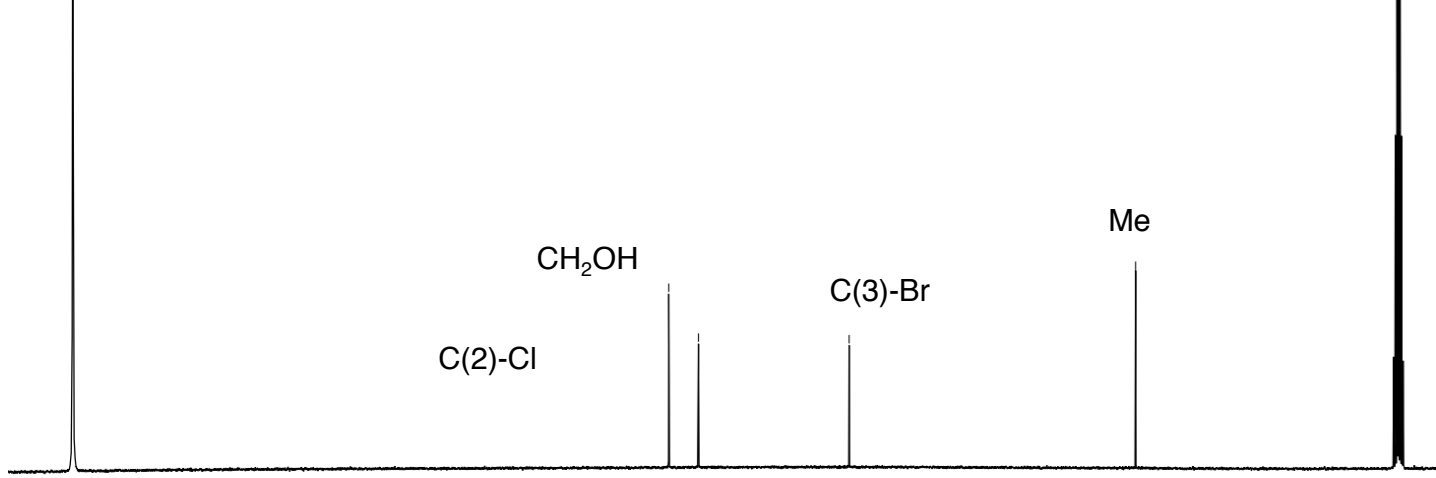

$\begin{array}{llllllllllllllllllllllllll}120 & 115 & 110 & 105 & 100 & 95 & 90 & 85 & 80 & 75 & 70 & 65 & 60 & 55 & 50 & 45 & 40 & 35 & 30 & 25 & 20 & 15 & 10 & 5 & 0\end{array}$ f1 (ppm)

Figure S83. ${ }^{13} \mathrm{C}$ NMR $(150 \mathrm{MHz})$ traces of $\left(R^{*}, S^{*}\right)-7$ in acetonitrile- $d_{3}$ at $298 \mathrm{~K}$.

\section{$600 \mathrm{MHz}$ NMR}

acetonitrile- $d_{3}$

${ }^{1} \mathrm{H}$

$298 \mathrm{~K}$

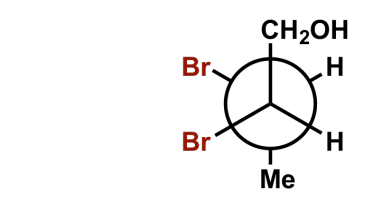

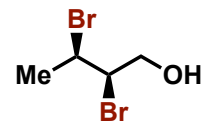

$( \pm)$

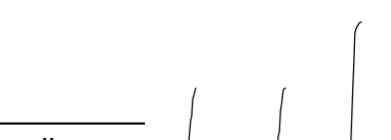

\begin{tabular}{|c|c|c|c|c|}
\hline${ }^{3} \mathrm{~J}(\mathrm{H} 2, \mathrm{H} 3)$ & $+2.5 \mathrm{~Hz}$ & small & & \\
\hline${ }^{3} J(\mathrm{H} 2, \mathrm{C} 4)$ & $+2.7 \mathrm{~Hz}$ & small & & \\
\hline${ }^{3} J(\mathrm{H} 3, \mathrm{C} 1)$ & & & & \begin{tabular}{|c}
$C$ (ddd) \\
4.16
\end{tabular} \\
\hline${ }^{2} J(\mathrm{H} 2, \mathrm{C} 3)$ & $+1.6 \mathrm{~Hz}$ & small & $\begin{array}{c}A(q d) \\
4.60\end{array}$ & \\
\hline $2 J(H 3, C 2)$ & $+1.4 \mathrm{~Hz}$ & small & & \\
\hline
\end{tabular}

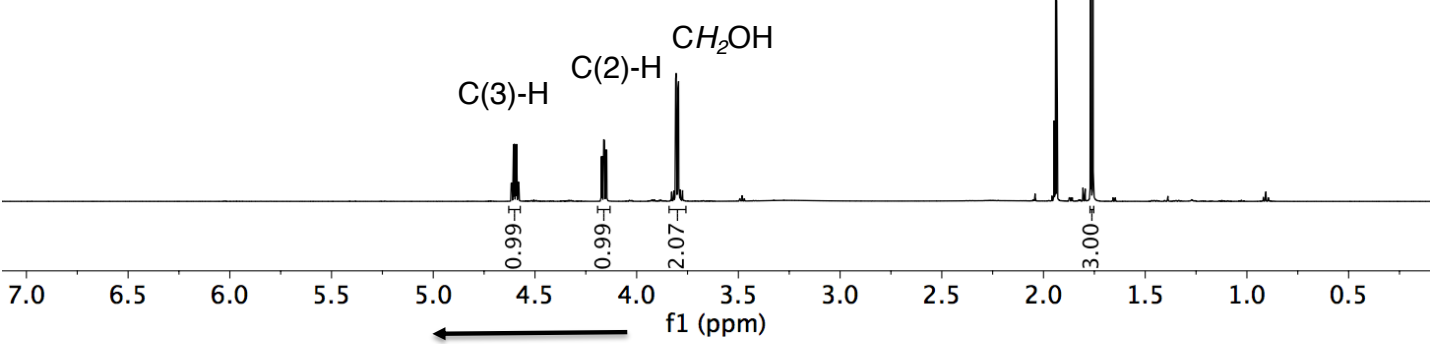

Figure S84. ${ }^{1} \mathrm{H}$ NMR $(600 \mathrm{MHz})$ traces of $\left(R^{*}, R^{*}\right)-8$ in acetonitrile- $d_{3}$ at $298 \mathrm{~K} . J$-based coupling constants are assigned and given in the table. 


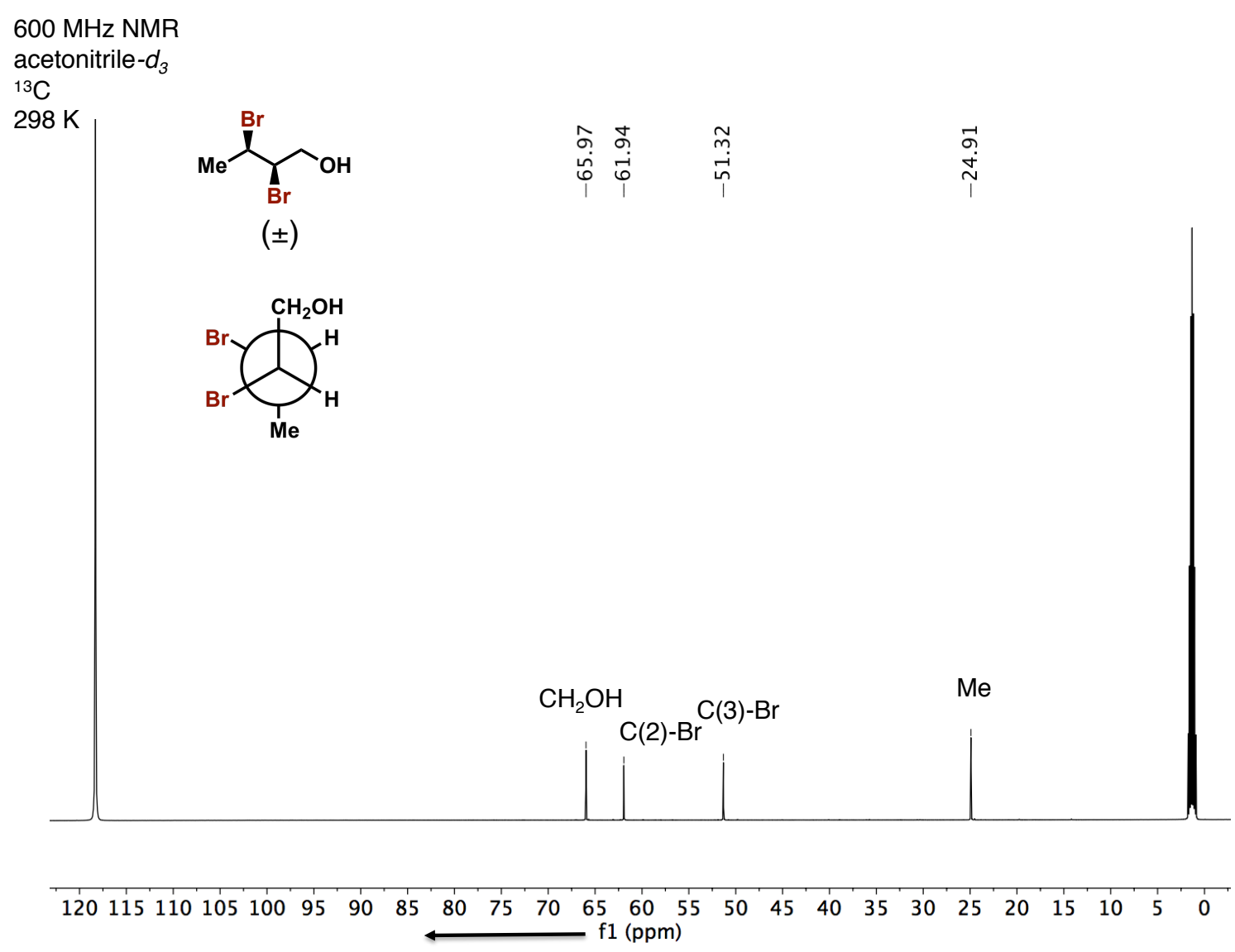

Figure S85. ${ }^{13} \mathrm{C}$ NMR $(150 \mathrm{MHz})$ traces of $\left(R^{*}, R^{*}\right)-8$ in acetonitrile- $d_{3}$ at $298 \mathrm{~K}$.

\section{S11. Structures Obtained from Single Crystal X-ray Diffraction}

\section{General Crystallization Protocol:}

Enantiopure AAC $(P)_{4}-\mathbf{1}$ or $(M)_{4}-\mathbf{1}(\sim 1 \mathrm{mg})$ were dissolved in dry acetonitrile $(1 \mathrm{~mL})$ in a conical vials $(1.5 \mathrm{~mL})$ and the non-crystalline guest was added $(\sim 1-2 \mathrm{mg})$. The vial was sealed and cooled from $20^{\circ} \mathrm{C}$ to $0{ }^{\circ} \mathrm{C}$ over $72-100 \mathrm{~h}$ using a PID controller to establish a linear profile. The single crystals were subsequently submerged in perfluoroalkylether oil and left for ca. $48 \mathrm{~h}$ (to allow for phase transition into the orthorhombic polymorph). Suitable single crystals were then studied by single crystal X-ray diffraction.

All X-ray co-crystal structures presented herein (CCDC 1914836-1914859) crystallized in space group $P 2{ }_{1} 2_{1} 2_{1}$ or $P 2{ }_{1}$. These usually exhibited only one cavity occupied by a guest molecule. Occasionally, they showed disorder in the top hydrogen-bonding array and alkyl periphery, corresponding with overall decreasing structure quality. Occasionally, monoclinic structures were found, but these crystals reliably and irreversibly relaxed into the orthorhombic polymorph. For this compound class, the presented X-ray co-crystal structures show comparatively high resolution and low $R$ values, meeting generally accepted small molecule 
crystal structure publication standards. In the following images, only the donor hydrogen atoms are shown for clarity.

\section{Comparison of X-ray Co-crystal structures of $A A C(P)_{4}-1$ with Aliphatic Alcohols}

A

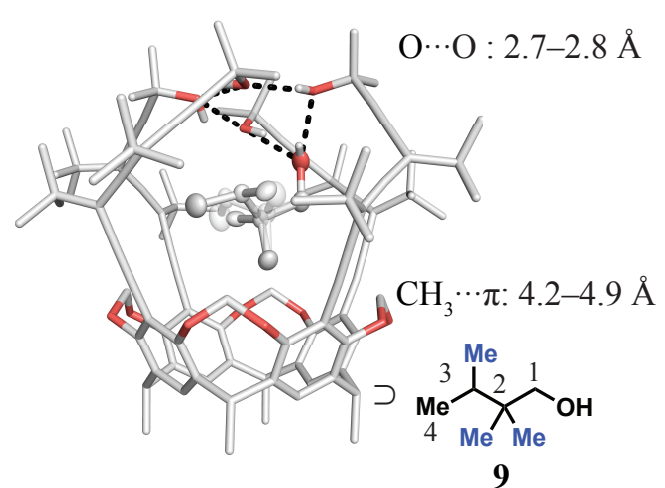

B

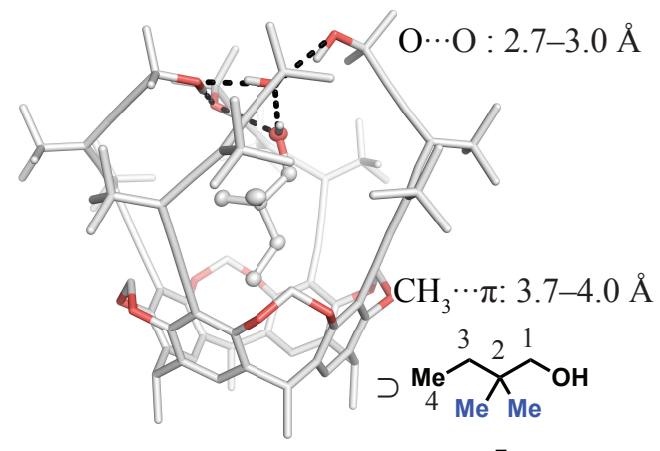

5

Host-bound conformers:

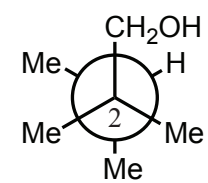

Relative occupancy:

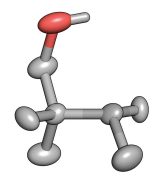

$60 \%$

$42 \%$<smiles>O=CC1CCCCC1C(=O)O</smiles>

9

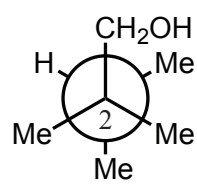

$40 \%$

$58 \%$

Figure S86. X-ray co-crystal structure of AAC $(P)_{4-1}$ with 2,2,3-trimethylbutanol $\mathbf{9}(\mathbf{A})$; the guest is bound in two enantiomeric conformations. X-ray co-crystal structure of AAC $(P)_{4}-\mathbf{1}$ with 2,2-dimethylbutanol 5 (B). Distances are given in $\AA$. $n$-Hexyl chains of the receptor are omitted for clarity. Guests are shown in their ellipsoid representation $(50 \%$ probability). 


\section{Alcohols}

A

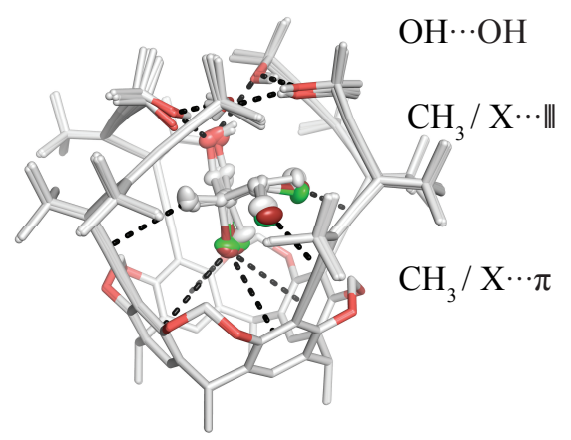

B

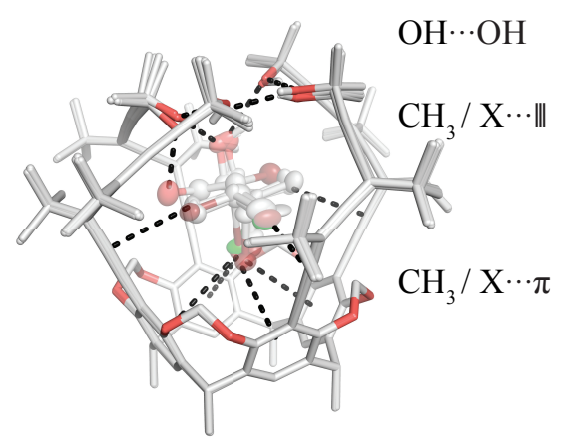

Host-bound guests:<smiles>C[C@H](Cl)[C@H](Cl)CO</smiles>

$(R, S)-7$

$(R, R)-7$<smiles>C[13CH]C([18Br])CO</smiles><smiles>OC[C@H](Br)C(Br)Br</smiles>

$(R, S)-8$

$(R, R)-8$

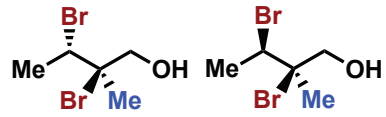

$(R, S)-10$

$(R, R)-10$<smiles>OC[C@H](Cl)C(Cl)Cl</smiles>

$(S, R)-7$

Figure S87. Overlay of X-ray co-crystal structures obtained from AAC $(P)_{4}-1$ with the selected series of racemic guest molecules. Preferential complexation of the guest enantiomers was observed: The stronger binding guest enantiomers (higher occupancy) are depicted in $\mathbf{A}$, while the weaker binding (lower occupancy) are depicted in $\mathbf{B}$ with decreased transparency of the ellipsoids. $n$-Hexyl chains of the receptor are omitted for clarity. Guests are shown in their ellipsoid representation (50\% probability).

X-ray Co-Crystal Structure as Determined by Single Crystal X-Ray Diffraction: $(P)_{4}-$ AACつ2,2-Dimethylbutan-1-ol 5: CCDC-1914838

Single crystals were grown following the general crystallization protocol. All protons were detected crystallographically. The H-bonding array shows a clockwise orientation. 


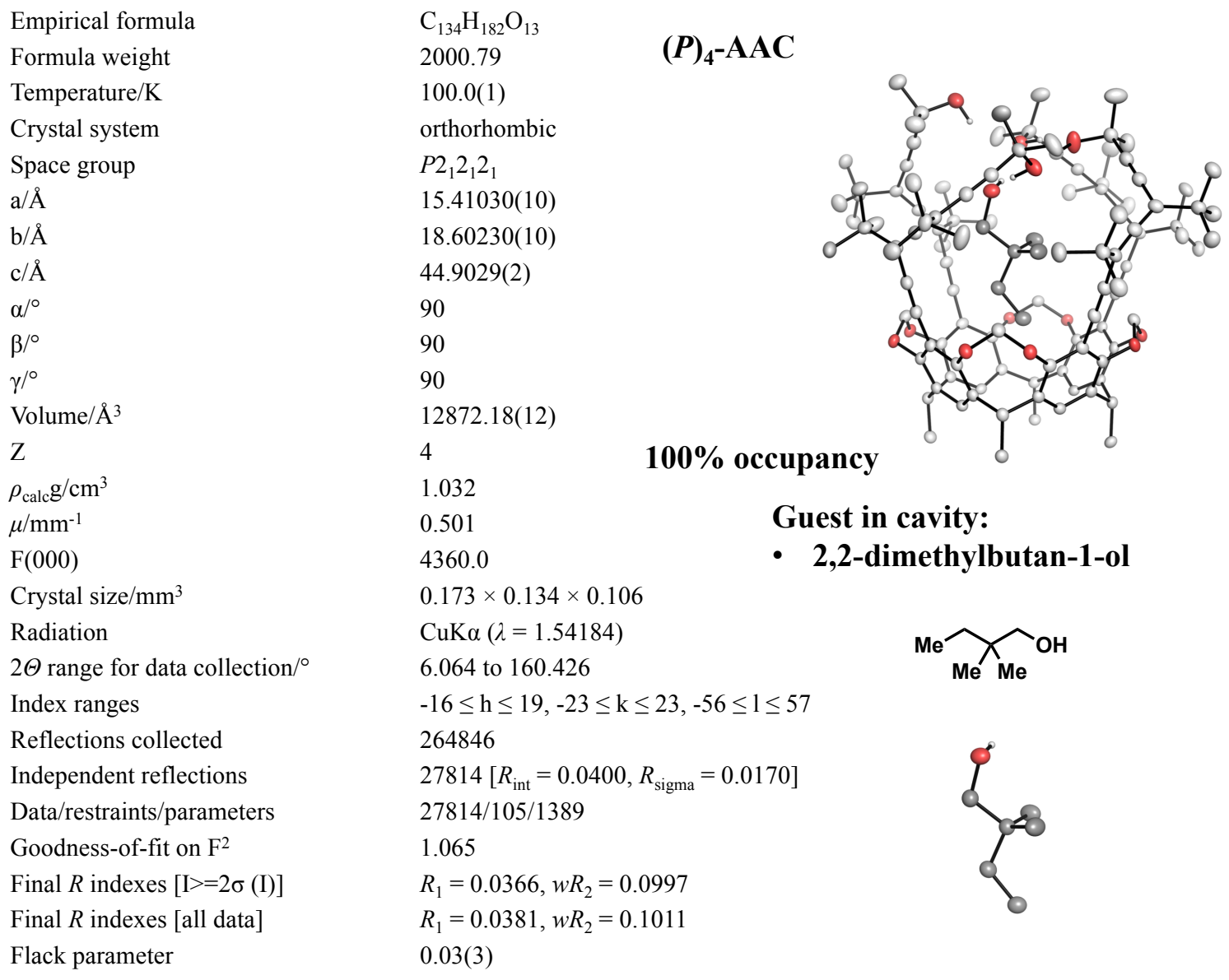

Figure S88. Crystallographic data and ORTEP representation of $(P)_{4}$-AAC $\supset 2,2$-dimethylbutan-1-ol 5. Ellipsoids are shown at $50 \%$ probability. $n$-Hexyl chains are omitted for clarity.

X-ray Co-Crystal Structure as Determined by Single Crystal X-Ray Diffraction: $(P)_{4}-$ AACつ2,2,3-Trimethylbutan-1-ol 9: CCDC-1914840

Single crystals were grown following the general crystallization protocol. The guest 9 is disordered over two positions with major and minor occupancies of $60 \%$ and $40 \%$. The different occupancy of the guest molecule corresponds to the two enantiomeric conformers depicted below. The sample was twinned, but the domains could be cleanly separated during raw data integration. HKLF5 format structure factor data was employed during final refinement. Absolute structure could not be determined from diffraction data in this case, which is of no consequence since the absolute configuration of the AAC is known. 


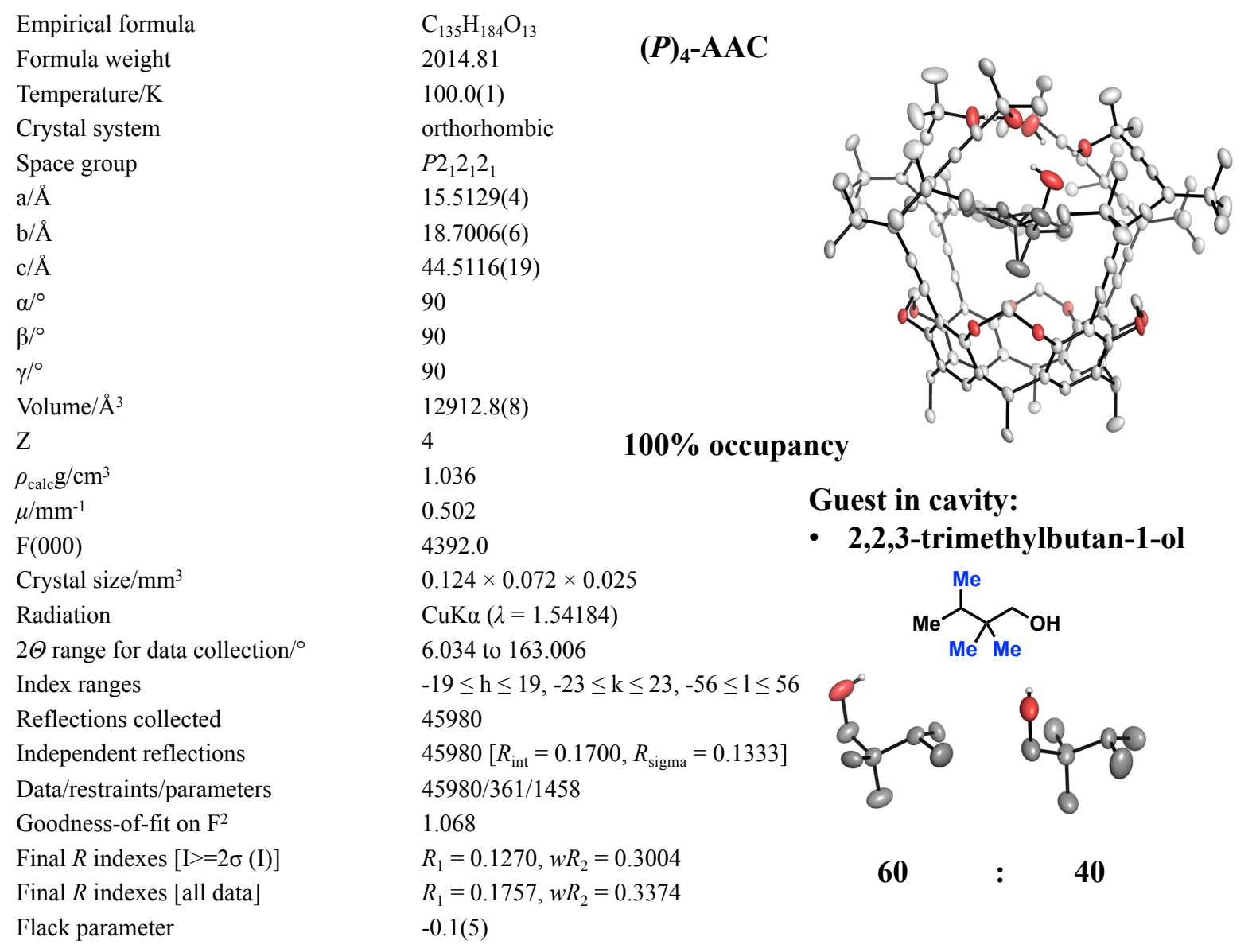

Figure S89. Crystallographic data and ORTEP representation of $(P)_{4}$-AAC $\supset 2,2,3$-trimethylbutan-1-ol 9. Ellipsoids are shown at 50\% probability. $n$-Hexyl chains are omitted for clarity.

X-ray Co-Crystal Structure as Determined by Single Crystal X-Ray Diffraction: $(M)_{4}-$ AAC $\supset$ 2,2,3-Trimethylbutan-1-ol 9: CCDC-1914836

Single crystals were grown following the general crystallization protocol. All protons were detected crystallographically. The H-bonding array shows a counter-clockwise orientation. The guest 9 is disordered over two positions with major and minor occupancies of $58 \%$ and $42 \%$. The different occupancy of the guest molecule corresponds to the two enantiomeric conformers depicted below. Traces of solvent encapsulation in the AAC are visible in the difference density map, but proved too insignificant to refine in a stable convergent model. 


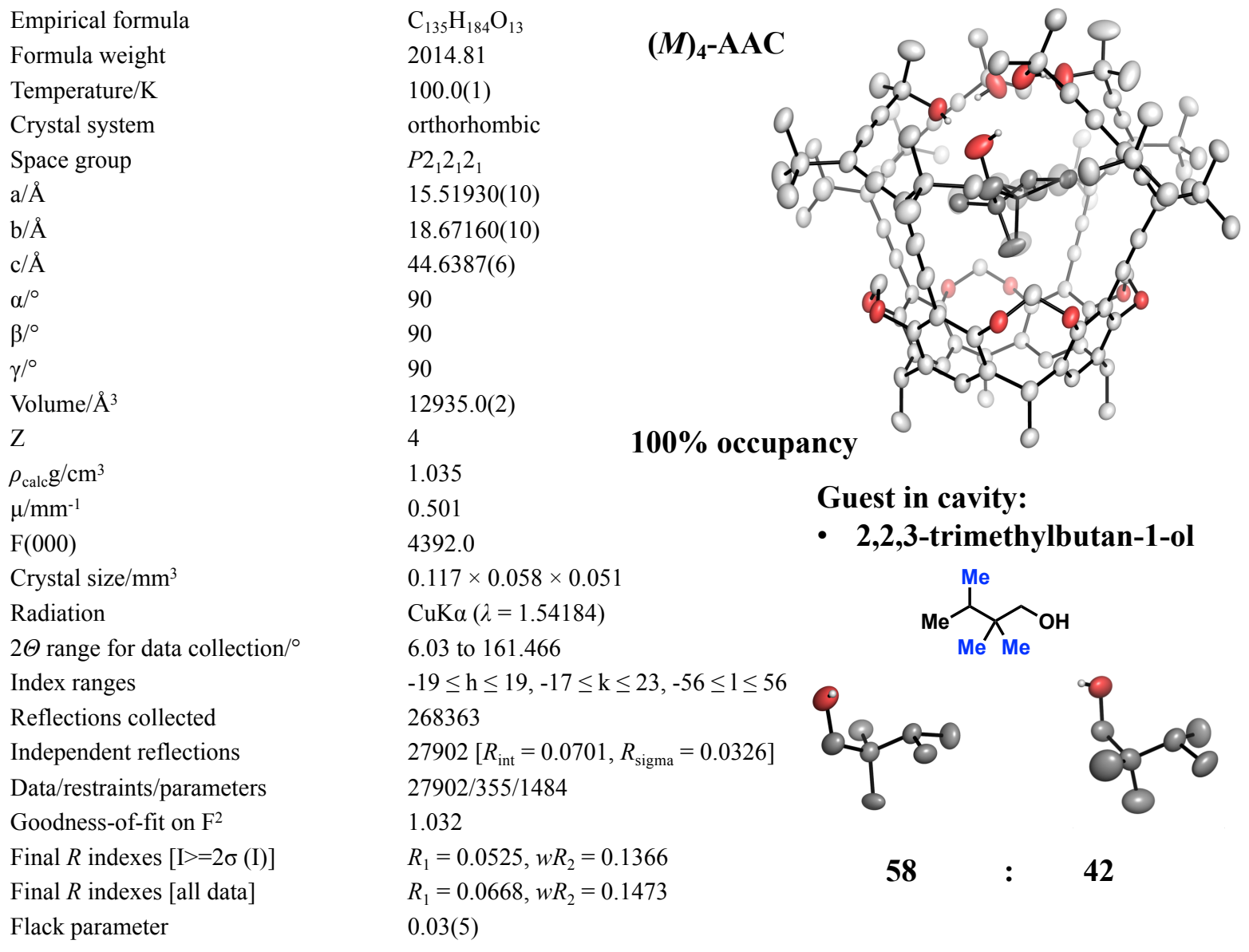

Figure S90. Crystallographic data and ORTEP representation of $(M)_{4}$-AAC $\supset 2,2,3$-trimethylbutan-1-ol 9. Ellipsoids are shown at $50 \%$ probability. $n$-Hexyl chains are omitted for clarity.

X-ray Co-Crystal Structure as Determined by Single Crystal X-Ray Diffraction: $(P)_{4}$ AAC $\supset( \pm)-\left(2 S^{*}, 3 R^{*}\right)-2,3-D i c h l o r o b u t a n-1-o l ~\left(R^{*}, S^{*}\right)-7:$ CCDC-1914858

Single crystals were grown following the general crystallization protocol. The guest $\left(R^{*}, S^{*}\right)-7$ is disordered over two positions with major and minor occupancies of $58 \%$ and $42 \%$. The different occupancy of the guest molecule corresponds to the two enantiomers depicted below. 


Empirical formula
Formula weight
Temperature/K
Crystal system
Space group
$\mathrm{a} / \AA$
$\mathrm{b} / \AA$
$\mathrm{c} / \AA$
$\alpha /{ }^{\circ}$
$\beta /{ }^{\circ}$
$\gamma /{ }^{\circ}$
Volume $/ \AA^{3}$
$\mathrm{Z}$
$\rho_{\text {calcg }} \mathrm{gm}{ }^{3}$
$\mu / \mathrm{mm}^{-1}$
$\mathrm{~F}(000)$
Crystal size/mm ${ }^{3}$
Radiation
$2 \Theta$ range for data collection ${ }^{\circ}$
Index ranges
Reflections collected
Independent reflections
Data/restraints $/$ parameters
Goodness-of-fit on $\mathrm{F}^{2}$
Final $R$ indexes $[\mathrm{I}>=2 \sigma(\mathrm{I})]$
Final $R$ indexes [all data $]$
Flack parameter

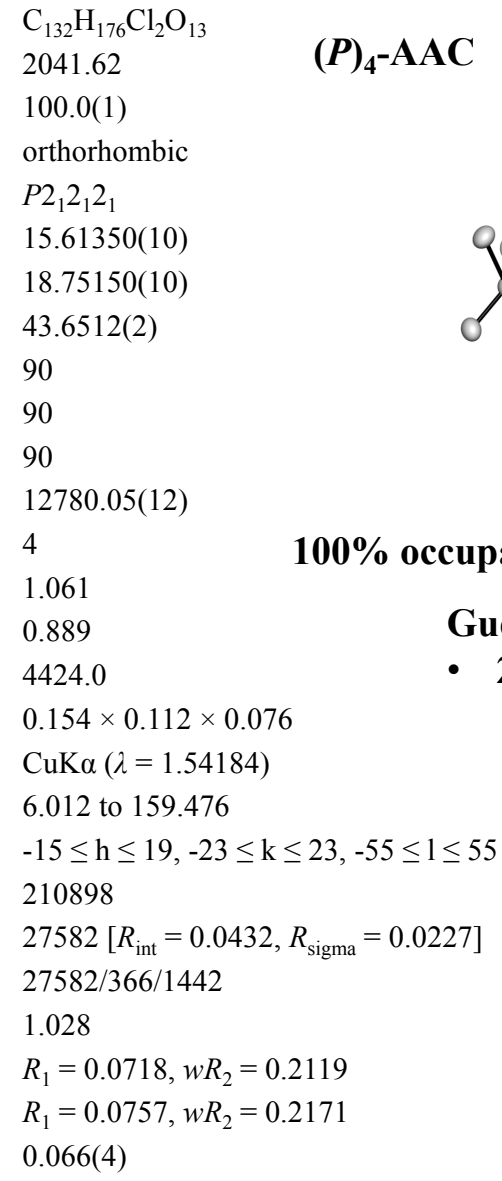

$(P)_{4}$-AAC

$100 \%$ occupancy

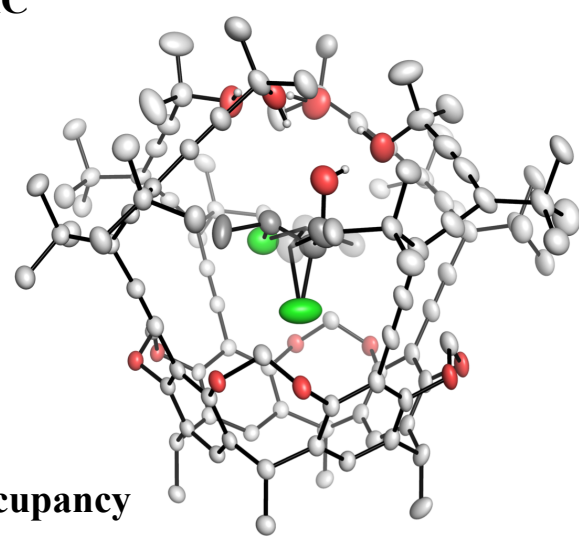

Guest in cavity:

- 2,3-dichlorobutan-1-ol<smiles>C[C@H](Cl)[C@H](Cl)CO</smiles><smiles>C[C@H](Cl)[C@H](Cl)CO</smiles>
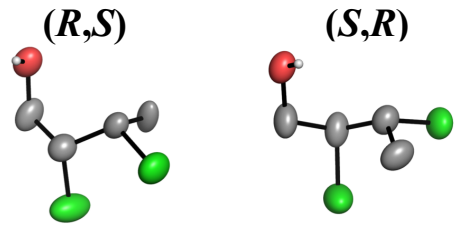

58 : 42

Figure S91. Crystallographic data and ORTEP representation of $(P)_{4}$-AAC $\supset( \pm)-\left(2 S^{*}, 3 R^{*}\right)$-2,3-dichlorobutan-1ol $\left(R^{*}, S^{*}\right)-7$. Ellipsoids are shown at $50 \%$ probability. $n$-Hexyl chains are omitted for clarity.

X-ray Co-Crystal Structure as Determined by Single Crystal X-Ray Diffraction: $(M)_{4}$ AAC $\supset( \pm)-\left(2 S^{*}, 3 R^{*}\right)-2,3-D i c h l o r o b u t a n-1-o l ~\left(R^{*}, S^{*}\right)-7:$ CCDC-1914853

Single crystals were grown following the general crystallization protocol. All protons were detected crystallographically. The guest $\left(R^{*}, S^{*}\right)-7$ is disordered over two positions with major and minor occupancies of $53 \%$ and $47 \%$. The different occupancy of the guest molecule corresponds to the two enantiomers depicted below. 


Empirical formula
Formula weight
Temperature/K
Crystal system
Space group
$\mathrm{a} / \AA$
$\mathrm{b} / \AA$
$\mathrm{c} / \AA$
$\alpha /{ }^{\circ}$
$\beta /{ }^{\circ}$
$\gamma /{ }^{\circ}$
Volume/ $\AA^{3}$
$\mathrm{Z}$
$\rho_{\text {calc }} \mathrm{g} / \mathrm{cm}^{3}$
$\mu / \mathrm{mm}^{-1}$
$\mathrm{~F}(000)$
Crystal size/mm ${ }^{3}$
Radiation
$2 \Theta$ range for data collection/ ${ }^{\circ}$
Index ranges
Reflections collected
Independent reflections
Data/restraints/parameters
Goodness-of-fit on $\mathrm{F}^{2}$
Final $R$ indexes $[\mathrm{I}>=2 \sigma(\mathrm{I})]$
Final $R$ indexes [all data $]$
Flack parameter
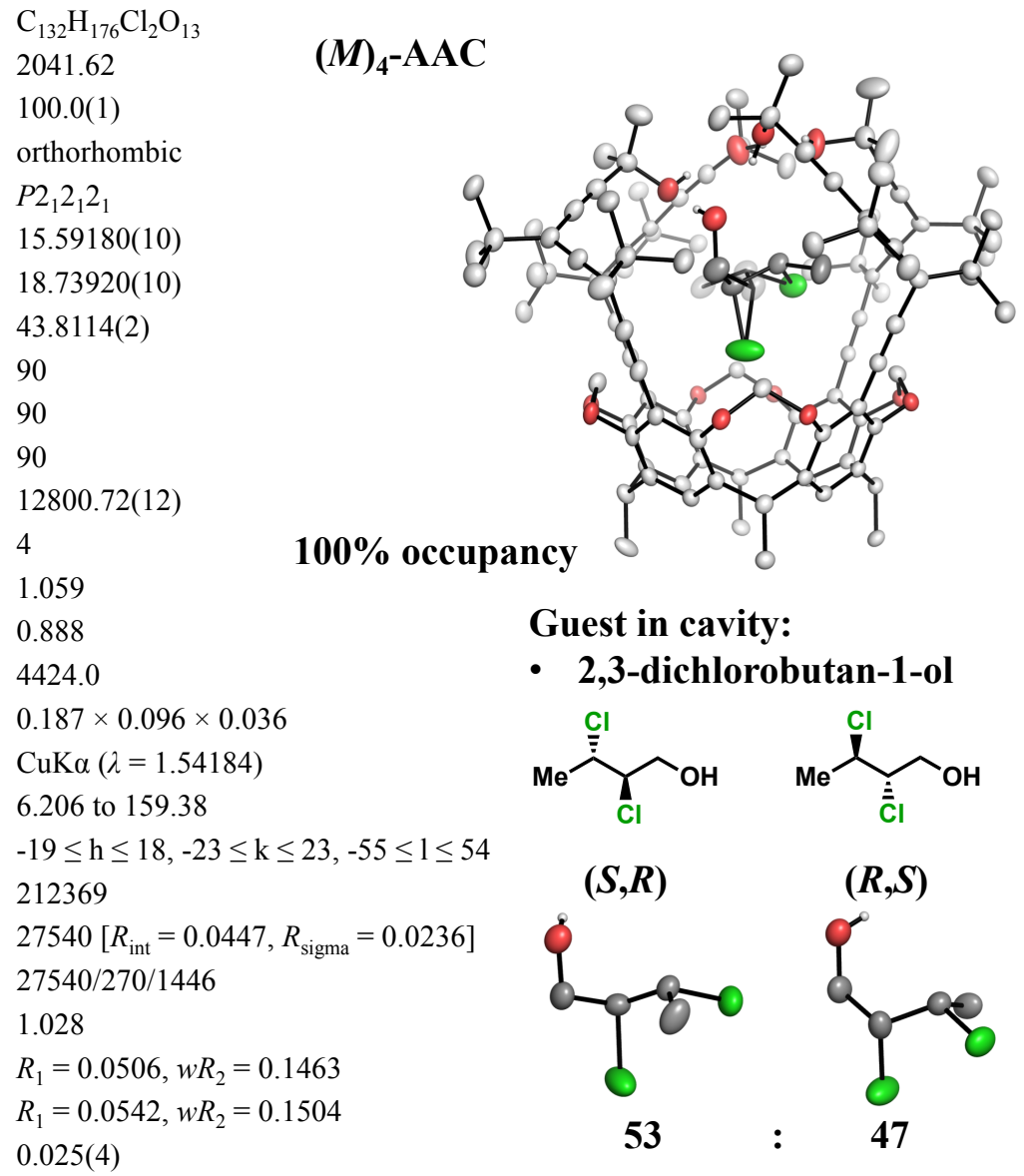

Guest in cavity:

- 2,3-dichlorobutan-1-ol

Figure S92. Crystallographic data and ORTEP representation of $(M)_{4}$-AAC $\supset( \pm)-\left(2 S^{*}, 3 R^{*}\right)$-2,3-dichlorobutan-1ol $\left(R^{*}, S^{*}\right)-7$. Ellipsoids are shown at $50 \%$ probability. $n$-Hexyl chains are omitted for clarity.

X-ray Co-Crystal Structure as Determined by Single Crystal X-Ray Diffraction: $(P)_{4}$ $\operatorname{AAC} \supset( \pm)-\left(2 R^{*}, 3 R^{*}\right)-2,3-D i c h l o r o b u t a n-1-o l ~\left(R^{*}, R^{*}\right)-7:$ CCDC-1914852

Single crystals were grown following the general crystallization protocol. The guest $\left(R^{*}, R^{*}\right)$ 7 shows $50 \%$ occupancy, with some indications in the difference density that the remaining occupancy consists of DCM. However, this could not be modelled. 


Empirical formula
Formula weight
Temperature/K
Crystal system
Space group
$\mathrm{a} / \AA$
$\mathrm{b} / \AA$
$\mathrm{c} / \AA$
$\alpha /{ }^{\circ}$
$\beta /{ }^{\circ}$
$\gamma /{ }^{\circ}$
Volume/ $\AA^{3}$
$\mathrm{Z}$
$\rho_{\text {calc }} \mathrm{g} / \mathrm{cm}^{3}$
$\mu / \mathrm{mm}^{-1}$
$\mathrm{~F}(000)$
Crystal size/mm ${ }^{3}$
Radiation
$2 \Theta$ range for data collection $/{ }^{\circ}$
Index ranges
Reflections collected
Independent reflections
Data/restraints/parameters
Goodness-of-fit on $\mathrm{F}^{2}$
Final $R$ indexes $[\mathrm{I}>=2 \sigma(\mathrm{I})]$
Final $R$ indexes [all data]
Flack parameter

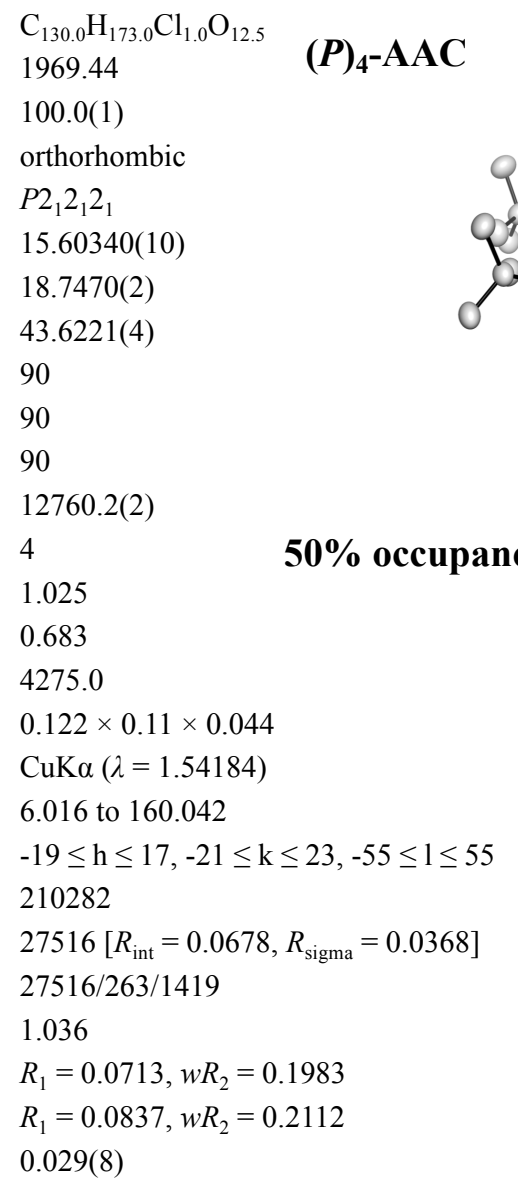

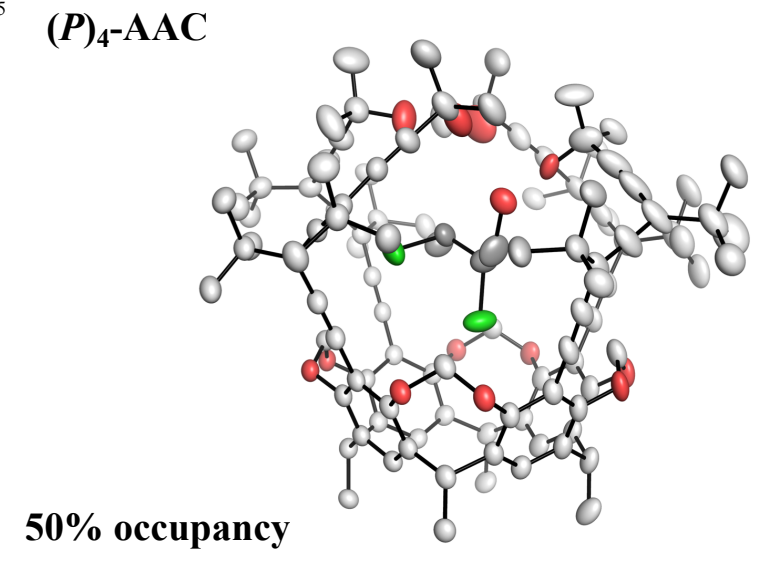

Guest in cavity:

- 2,3-dichlorobutan-1-ol<smiles>C[C@H](Cl)[C@H](Cl)CO</smiles>

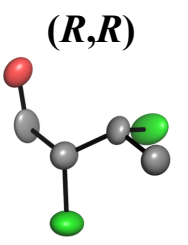

Figure S93. Crystallographic data and ORTEP representation of $(P)_{4}$-AAC $\supset( \pm)-\left(2 R^{*}, 3 R^{*}\right)$-2,3-dichlorobutan-1ol $\left(R^{*}, R^{*}\right)-7$. Ellipsoids are shown at $50 \%$ probability. $n$-Hexyl chains are omitted for clarity.

\section{X-ray Co-Crystal Structure as Determined by Single Crystal X-Ray Diffraction: $(M)_{4}$ -}

\section{AAC $\supset( \pm)-\left(2 R^{*}, 3 R^{*}\right)-2,3-D i c h l o r o b u t a n-1-o l ~\left(R^{*}, R^{*}\right)-7$ : CCDC-1914855}

Single crystals were grown following the general crystallization protocol. The guest $\left(R^{*}, R^{*}\right)$ 7 shows $70 \%$ occupancy. The remaining cavities are occupied by an alternate conformation of the guest molecule with the hydroxyl group pointing toward the cage walls, but this species could not be refined within a stable and convergent model. 


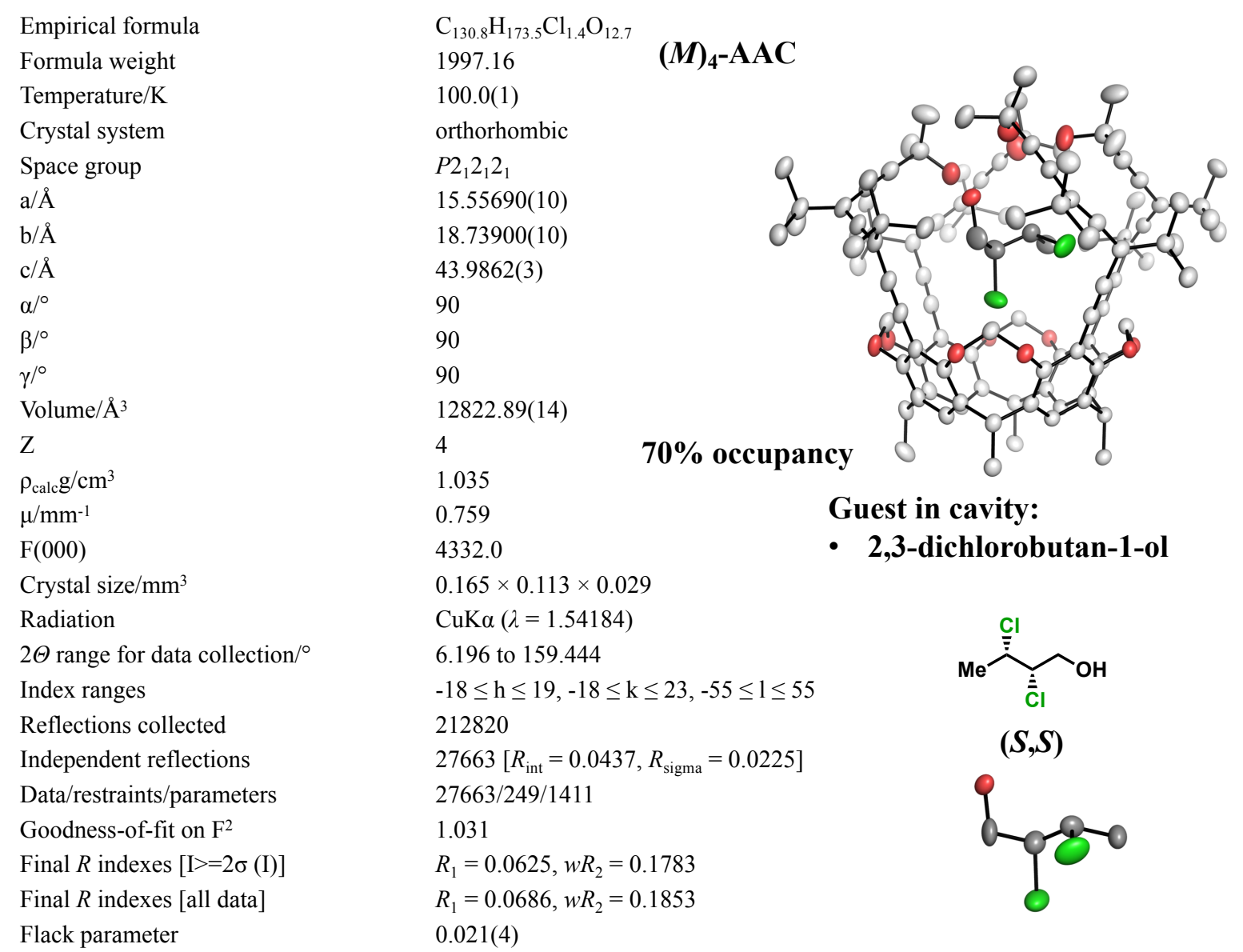

Figure S94. Crystallographic data and ORTEP representation of $(M)_{4}$-AAC $\supset( \pm)-\left(2 R^{*}, 3 R^{*}\right)-2,3$-dichlorobutan1 -ol $\left(R^{*}, R^{*}\right)-7$. Ellipsoids are shown at 50\% probability. $n$-Hexyl chains are omitted for clarity.

X-ray Co-Crystal Structure as Determined by Single Crystal X-Ray Diffraction: $(P)_{4}$ $\operatorname{AAC} \supset( \pm)-\left(2 S^{*}, 3 R^{*}\right)-2,3-D i b r o m o b u t a n-1-o l ~\left(R^{*}, S^{*}\right)-8:$ CCDC-1914857

Single crystals were grown following the general crystallization protocol. The guest $\left(R^{*}, S^{*}\right)-8$ is disordered over two positions with occupancies of 50\%. The different occupancy of the guest molecule corresponds to the two enantiomers depicted below. Hydrogen bonding pattern direction was chosen arbitrarily as residual peaks for protons were not detected. There are weak residual peaks clearly matching an alternate rotamer or different guest. Other possible reasons such as twinning or poor data could be excluded. In this alternate structure the hydrogen bonding array is clearly fully closed (only one host hydroxyl group is affected), equidistant and planar, i.e. no hydroxyl group of the guest impurity points towards the array. However, since the contribution is weak ( $5 \%$ or less), all refinement attempts failed and the alternate orientations were omitted from the model. An additional structure, in which two orientations of the guest molecule amount to only $60 \%$ occupancy (with residual electron density too diffuse to identify) is deposited under CCDC-1914843. 


Empirical formula
Formula weight
Temperature/K
Crystal system
Space group
$\mathrm{a} / \AA$
$\mathrm{b} / \AA$
$\mathrm{c} / \AA$
$\alpha{ }^{\circ}$
$\beta /{ }^{\circ}$
$\gamma /{ }^{\circ}$
Volume/ $\AA^{3}$
$\mathrm{Z}$
$\rho_{\text {calc }} \mathrm{g} / \mathrm{cm}^{3}$
$\mu / \mathrm{mm}^{-1}$
$\mathrm{~F}(000)$
Crystal size/mm ${ }^{3}$
Radiation
$2 \Theta$ range for data collection $/{ }^{\circ}$
Index ranges
Reflections collected
Independent reflections
Data/restraints/parameters
Goodness-of-fit on $\mathrm{F}^{2}$
Final $R$ indexes $[\mathrm{I}>=2 \sigma(\mathrm{I})]$
Final $R$ indexes $[$ all data $]$
Flack parameter

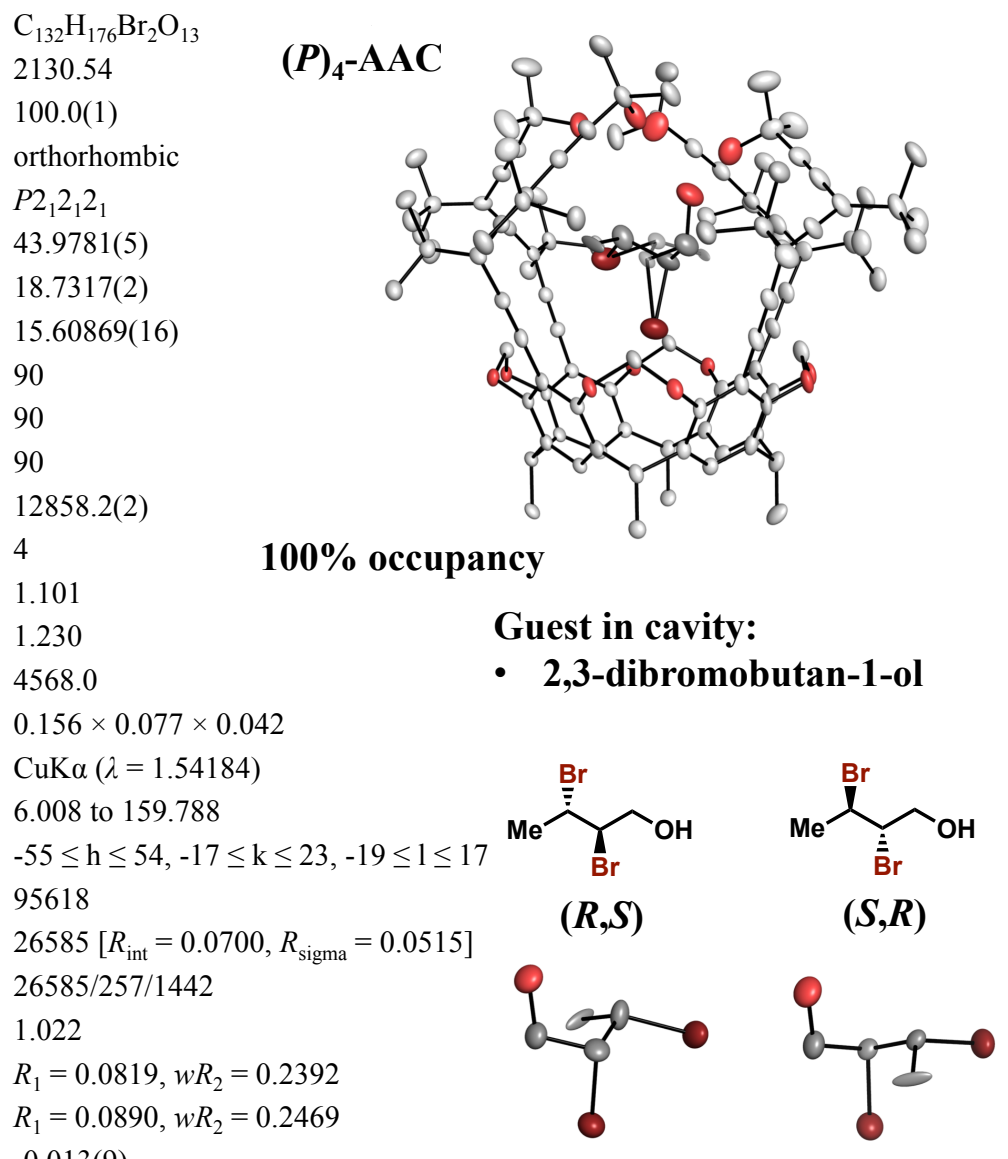

Figure S95. Crystallographic data and ORTEP representation of $(P)_{4}$-AAC $\supset( \pm)-\left(2 S^{*}, 3 R^{*}\right)-2,3-$ dibromobutan-1ol $\left(R^{*}, S^{*}\right)$-8. Ellipsoids are shown at $50 \%$ probability. $n$-Hexyl chains are omitted for clarity.

\section{X-ray Co-Crystal Structure as Determined by Single Crystal X-Ray Diffraction: $(M)_{4}$ -} AAC $\supset( \pm)-\left(2 S^{*}, 3 R^{*}\right)-2,3-D i b r o m o b u t a n-1-o l ~\left(R^{*}, S^{*}\right)-8:$ CCDC-1914841

Single crystals were grown following the general crystallization protocol. The guest $\left(R^{*}, S^{*}\right)-8$ is disordered over two positions with major and minor occupancies of $56 \%$ and $44 \%$. The different occupancy of the guest molecule corresponds to the two enantiomers depicted below. All hydrogen positions were detected crystallographically. There are weak residual peaks clearly matching two alternate guest rotamers or different guest species. Other possible reasons such as twinning or poor data could be excluded. However, since the contribution is weak $(5 \%$ or less), all refinement attempts failed and the alternate orientations were omitted from the model. 


\begin{tabular}{|c|}
\hline Empirical formula \\
\hline Formula weight \\
\hline Temperature/K \\
\hline Crystal system \\
\hline Space group \\
\hline $\mathrm{a} / \AA$ \\
\hline $\mathrm{b} / \AA$ \\
\hline $\mathrm{c} / \AA \AA$ \\
\hline$\alpha /^{\circ}$ \\
\hline$\beta /{ }^{\circ}$ \\
\hline$\gamma /{ }^{\circ}$ \\
\hline Volume $/ \AA^{3}$ \\
\hline $\mathrm{Z}$ \\
\hline $\begin{array}{l}\rho_{\text {calc }} \mathrm{g} / \mathrm{cm}^{3} \\
\mu / \mathrm{mm}^{-1}\end{array}$ \\
\hline $\mathrm{F}(000)$ \\
\hline Crystal size $/ \mathrm{mm}^{3}$ \\
\hline Radiation \\
\hline $2 \Theta$ range for data collection $/{ }^{\circ}$ \\
\hline Index ranges \\
\hline Reflections collected \\
\hline Independent reflections \\
\hline Data/restraints/parameters \\
\hline Goodness-of-fit on $\mathrm{F}^{2}$ \\
\hline Final $R$ indexes $[\mathrm{I}>=2 \sigma(\mathrm{I})]$ \\
\hline Final $R$ indexes [all data] \\
\hline Flack parameter \\
\hline
\end{tabular}

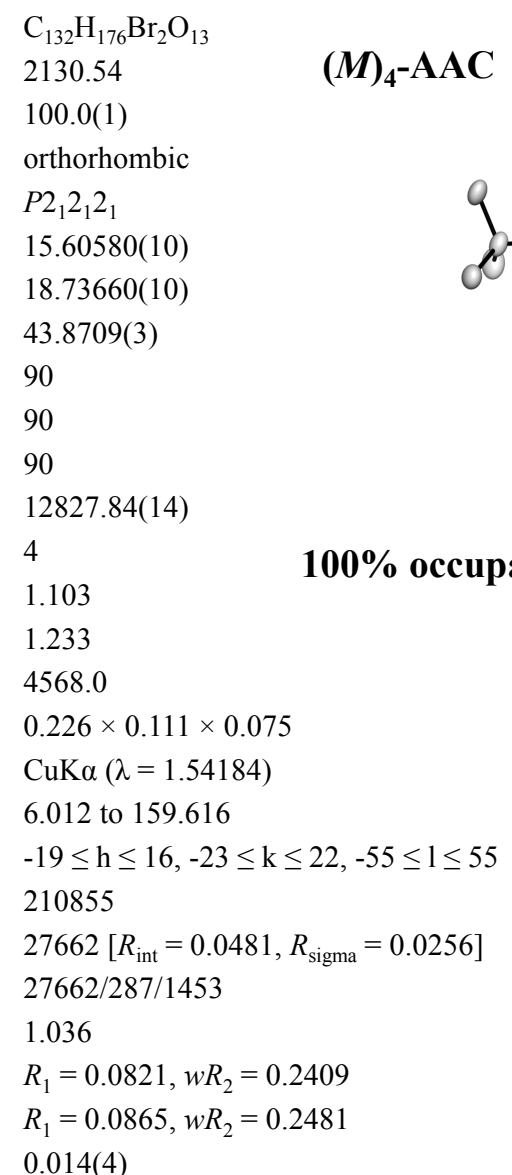

Figure S96. Crystallographic data and ORTEP representation of $(M)_{4}$-AAC $\supset( \pm)-\left(2 S^{*}, 3 R^{*}\right)-2,3$-dibromobutan-1ol $\left(R^{*}, S^{*}\right)-8$. Ellipsoids are shown at $50 \%$ probability. $n$-Hexyl chains are omitted for clarity.

\section{X-ray Co-Crystal Structure as Determined by Single Crystal X-Ray Diffraction: $(P)_{4}$ -}

\section{AAC $\supset( \pm)-\left(2 R^{*}, 3 R^{*}\right)-2,3-D i b r o m o b u t a n-1-o l ~\left(R^{*}, R^{*}\right)-8:$ CCDC-1914842}

Single crystals were grown following the general crystallization protocol. The guest $\left(R^{*}, R^{*}\right)$ 8 is disordered over three positions with major and minor occupancies of $68 \%, 24 \%$ and $8 \%$. The different occupancy of the guest molecule corresponds to the two enantiomers depicted below. 


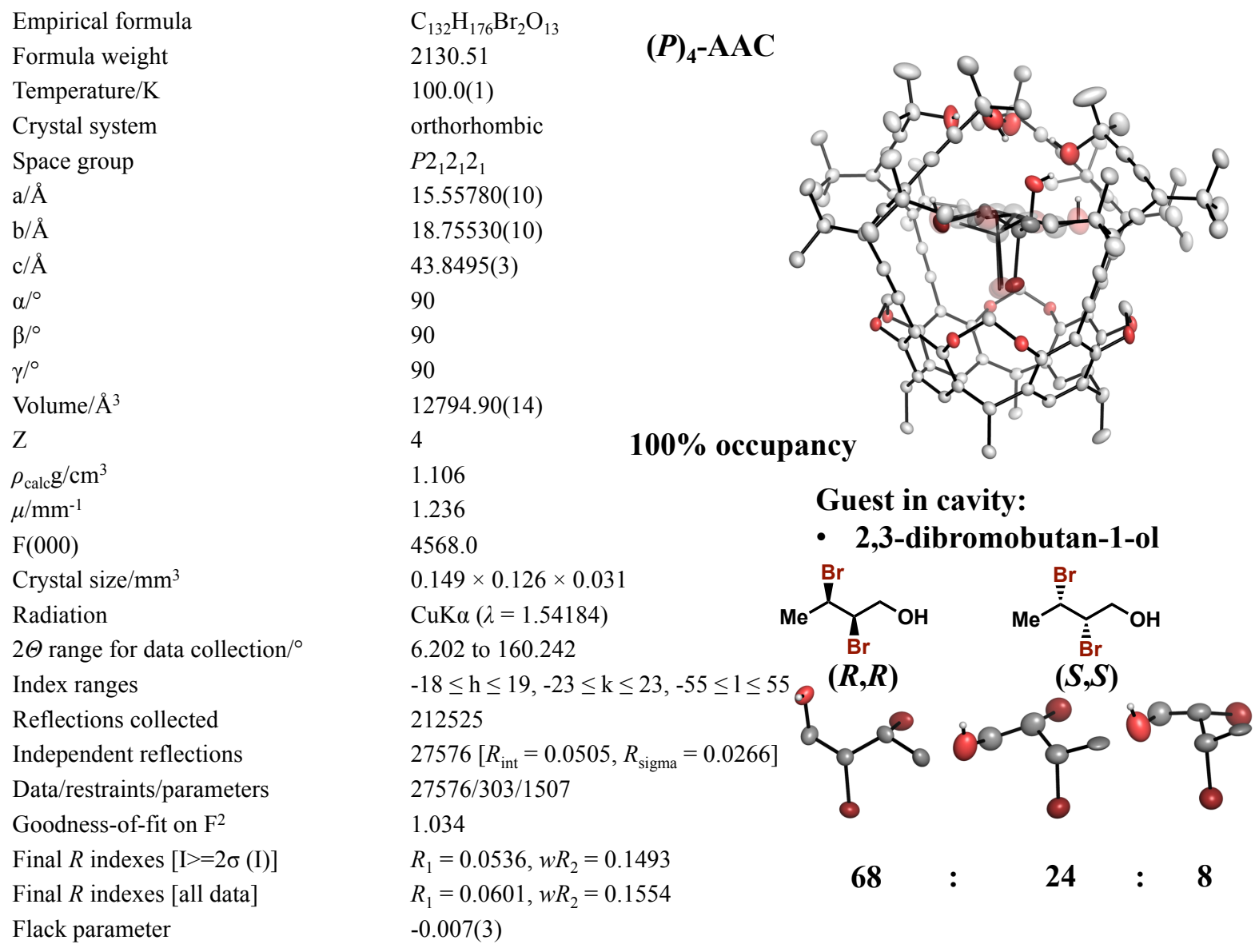

Figure S97. Crystallographic data and ORTEP representation of $(P)_{4}$-AAC $\supset( \pm)-\left(2 R^{*}, 3 R^{*}\right)$-2,3-dibromobutan-1ol $\left(R^{*}, R^{*}\right)$-8. Ellipsoids are shown at $50 \%$ probability. $n$-Hexyl chains are omitted for clarity.

X-ray Co-Crystal Structure as Determined by Single Crystal X-Ray Diffraction: $(M)_{4}-$ AAC $\supset( \pm)-\left(2 R^{*}, 3 R^{*}\right)-2,3-D i b r o m o b u t a n-1-o l ~\left(R^{*}, R^{*}\right)-8$ : CCDC-1914837

Single crystals were grown following the general crystallization protocol. The guest $\left(R^{*}, R^{*}\right)$ 8 shows $66 \%$ occupancy. The residual density was too diffuse to discern the remaining content. 


Empirical formula
Formula weight
Temperature/K
Crystal system
Space group
$\mathrm{a} / \AA$
$\mathrm{b} / \AA$
$\mathrm{c} / \AA$
$\alpha{ }^{\circ}$
$\beta /{ }^{\circ}$
$\gamma /{ }^{\circ}$
Volume/ $\AA^{3}$
$\mathrm{Z}$
$\rho_{\text {calc }} \mathrm{g} / \mathrm{cm}^{3}$
$\mu / \mathrm{mm}^{-1}$
$\mathrm{~F}(000)$
Crystal size/mm ${ }^{3}$
Radiation
$2 \Theta$ range for data collection ${ }^{\circ}$
Index ranges
Reflections collected
Independent reflections
Data/restraints/parameters
Goodness-of-fit on $\mathrm{F}^{2}$
Final $R$ indexes $[\mathrm{I}>=2 \sigma(\mathrm{I})]$
Final $R$ indexes [all data]
Flack parameter
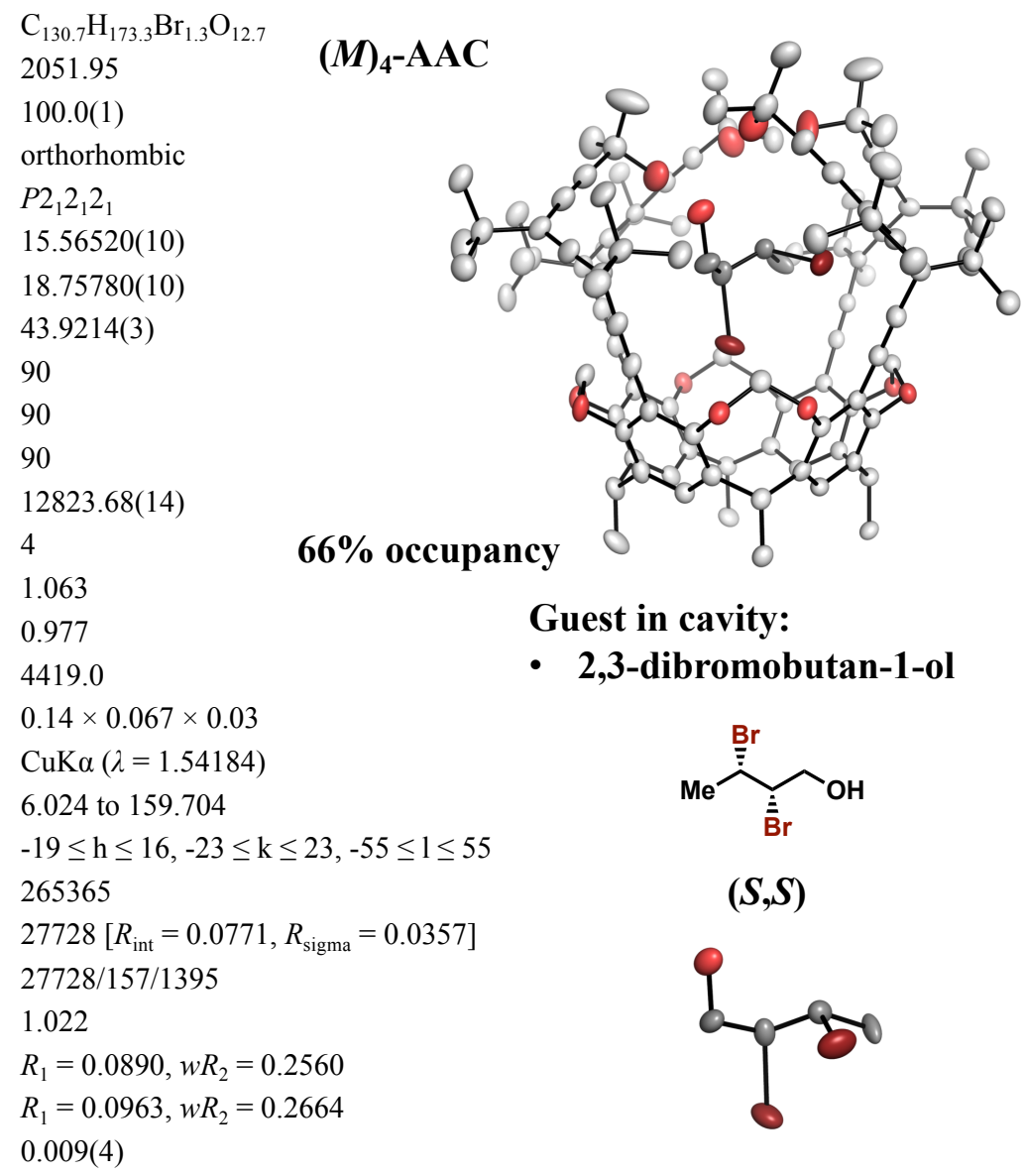

Guest in cavity:

- 2,3-dibromobutan-1-ol<smiles>C[C@@H](Br)[C@H](Br)CO</smiles>

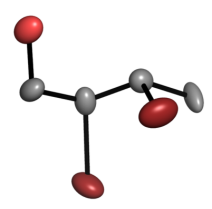

Figure S98. Crystallographic data and ORTEP representation of $(M)_{4}$-AAC $\supset( \pm)-\left(2 R^{*}, 3 R^{*}\right)-2,3$-dibromobutan1 -ol $\left(R^{*}, R^{*}\right)$-8. Ellipsoids are shown at $50 \%$ probability. $n$-Hexyl chains are omitted for clarity.

\section{X-ray Co-Crystal Structure as Determined by Single Crystal X-Ray Diffraction: $(P)_{4}-$}

\section{AAC $\supset( \pm)-\left(2 S^{*}, 3 R^{*}\right)-2,3-D i b r o m o-2-m e t h y l b u t a n-1-o l ~\left(R^{*}, S^{*}\right)-10$ : CCDC-1914839}

Single crystals were grown following the general crystallization protocol. The guest $\left(R^{*}, S^{*}\right)$ 10 is disordered over two positions with occupancies of 50\%. The different occupancy of the guest molecule corresponds to the two enantiomers depicted below. $15 \%$ of the host cavity is occupied by acetonitrile. This description does not incorporate a partial occupancy with acetonitrile in the host cavity, which is clearly visible in the difference density map and amounts to approximately $15 \%$. However, it was not possible to obtain a stable and convergent model including this contribution without over-constraining. 


Empirical formula
Formula weight
Temperature $/ \mathrm{K}$
Crystal system
Space group
$\mathrm{a} / \AA$
$\mathrm{b} / \AA$
$\mathrm{c} / \AA$
$\alpha{ }^{\circ}$
$\beta /{ }^{\circ}$
$\gamma /{ }^{\circ}$
Volume $/ \AA^{3}$
$\mathrm{Z}$
$\rho_{\text {calc }} \mathrm{g} / \mathrm{cm}^{3}$
$\mu / \mathrm{mm}{ }^{-1}$
$\mathrm{~F}(000)$
Crystal size/mm ${ }^{3}$
Radiation
$2 \Theta$ range for data collection$/{ }^{\circ}$
Index ranges
Reflections collected
Independent reflections
Data/restraints/parameters
Goodness-of-fit on $\mathrm{F}^{2}$
Final $R$ indexes $[\mathrm{I}>=2 \sigma(\mathrm{I})]$
Final $R$ indexes $[$ all data $]$
Flack parameter

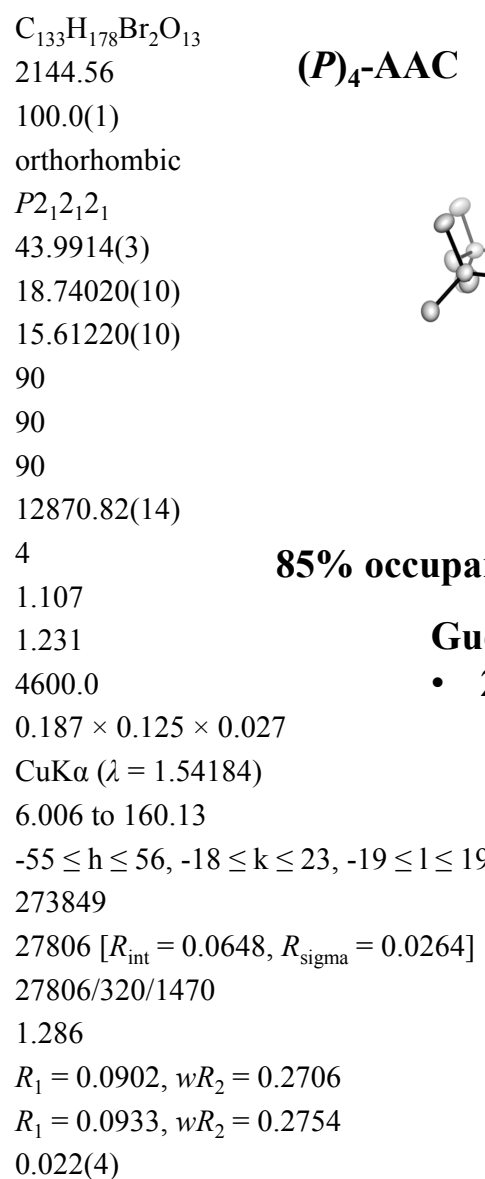

Figure S99. Crystallographic data and ORTEP representation of $(P)_{4}$-AAC $\supset( \pm)-\left(2 S^{*}, 3 R^{*}\right)-2,3$-Dibromo-2methylbutan-1-ol $\left(R^{*}, S^{*}\right)$-10. Ellipsoids are shown at 50\% probability. $n$-Hexyl chains are omitted for clarity.

X-ray Co-Crystal Structure as Determined by Single Crystal X-Ray Diffraction: $(M)_{4}$ AAC $\supset( \pm)-\left(2 S^{*}, 3 R^{*}\right)-2,3-D i b r o m o-2-m e t h y l b u t a n-1-o l ~\left(R^{*}, S^{*}\right)-10$ : CCDC-1914856

Single crystals were grown following the general crystallization protocol. The sample was twinned, but the domains could be cleanly separated during raw data integration. HKLF5 format structure factor data was employed during final refinement. The H-bonding array shows a clockwise orientation. The guest $\left(R^{*}, S^{*}\right)-\mathbf{1 0}$ is disordered over two positions with major and minor occupancies of $56 \%$ and $44 \%$. The different occupancy of the guest molecule corresponds to the two enantiomeric conformers depicted below. 


Empirical formula
Formula weight
Temperature/K
Crystal system
Space group
$\mathrm{a} / \AA$
$\mathrm{b} / \AA$
$\mathrm{c} / \AA$
$\alpha /{ }^{\circ}$
$\beta /{ }^{\circ}$
$\gamma /{ }^{\circ}$
Volume $/ \AA^{3}$
$\mathrm{Z}$
$\rho_{\text {calc }} \mathrm{g} / \mathrm{cm}^{3}$
$\mu / \mathrm{mm}^{-1}$
$\mathrm{~F}(000)$
Crystal size/mm ${ }^{3}$
Radiation
$2 \Theta$ range for data collection$/{ }^{\circ}$
Index ranges
Reflections collected
Independent reflections
Data/restraints/parameters
Goodness-of-fit on $\mathrm{F}^{2}$
Final $R$ indexes $[\mathrm{I}>=2 \sigma(\mathrm{I})]$
Final $R$ indexes [all data]
Flack parameter
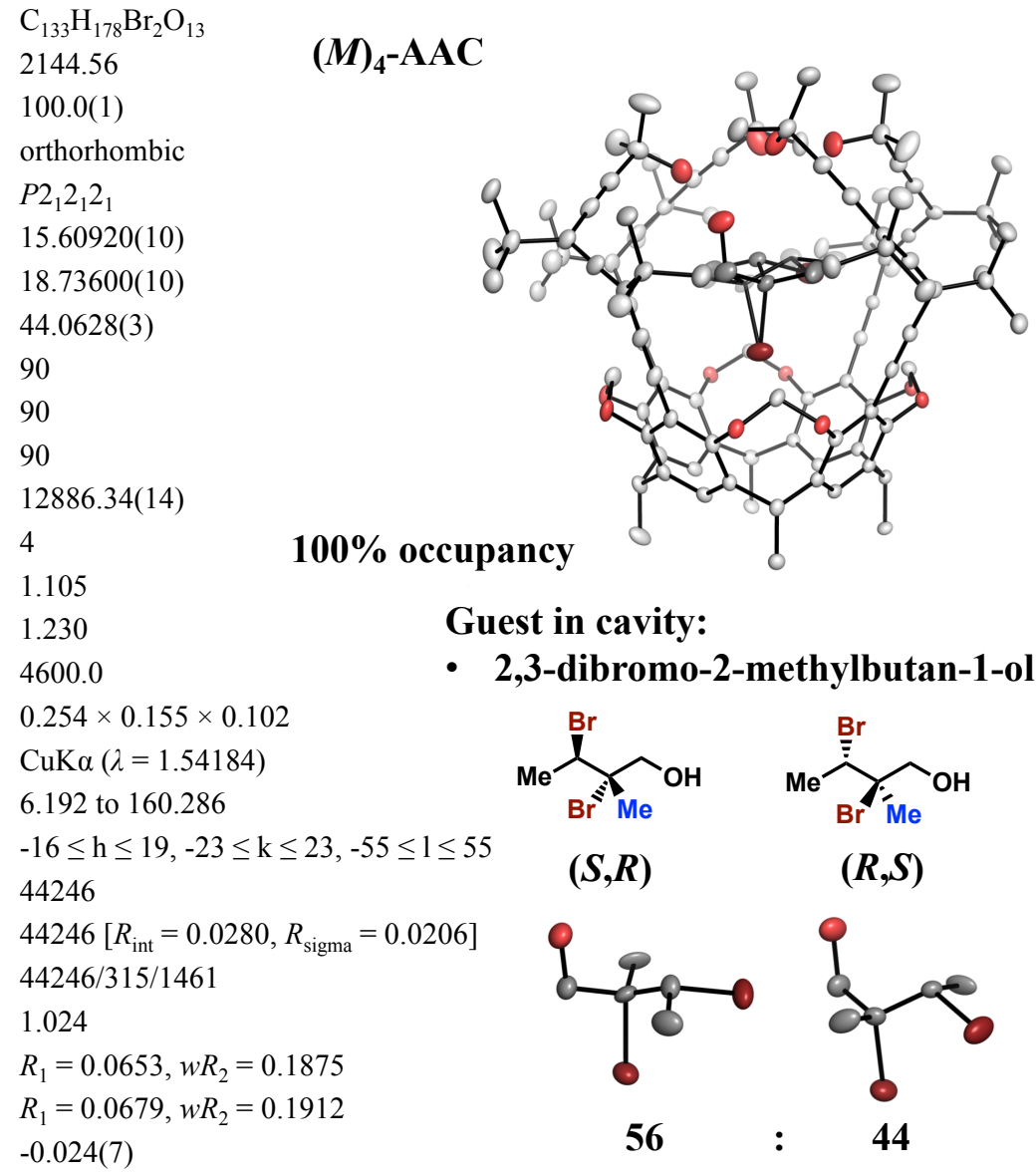

Guest in cavity:

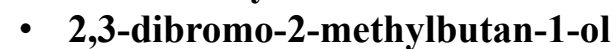

Figure S100. Crystallographic data and ORTEP representation of $(M)_{4}$-AAC $\supset( \pm)-\left(2 S^{*}, 3 R^{*}\right)-2,3$-Dibromo-2methylbutan-1-ol $\left(R^{*}, S^{*}\right)$-10. Ellipsoids are shown at 50\% probability. $n$-Hexyl chains are omitted for clarity.

X-ray Co-Crystal Structure as Determined by Single Crystal X-Ray Diffraction: $(P)_{4}$ AAC $\supset( \pm)-\left(2 R^{*}, 3 R^{*}\right)-2,3-D i b r o m o-2-m e t h y l b u t a n-1-o l ~\left(R^{*}, R^{*}\right)-10$ : CCDC-1914854

Single crystals were grown following the general crystallization protocol. All protons were detected crystallographically. The H-bonding array shows a clockwise orientation. The guest $\left(R^{*}, R^{*}\right)-\mathbf{1 0}$ is disordered over two positions with major and minor occupancies of $65 \%$ and $35 \%$. The different occupancy of the guest molecule corresponds to the two enantiomeric conformers depicted below. 


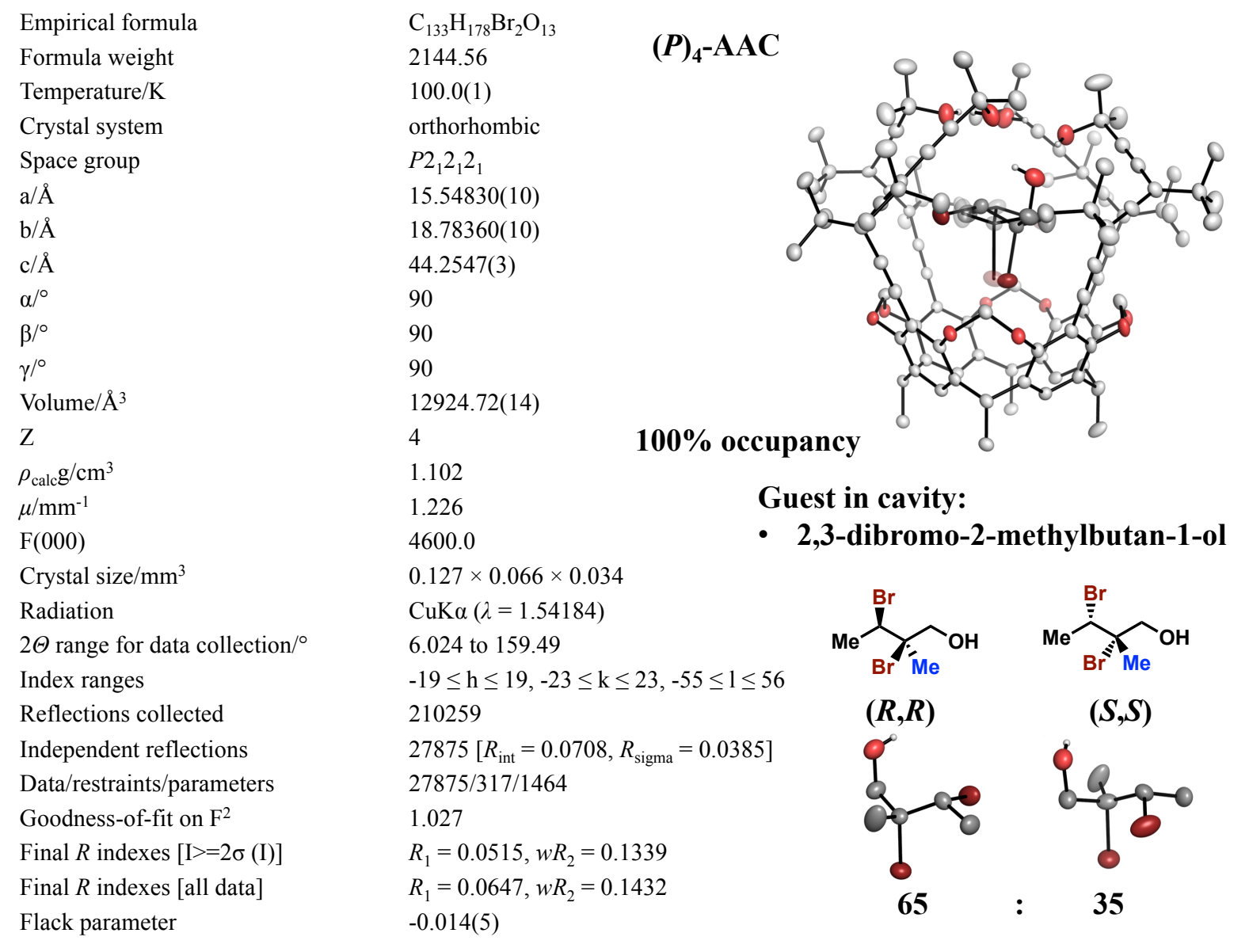

Figure S101. Crystallographic data and ORTEP representation of $(P)_{4}$-AAC $\supset( \pm)-\left(2 R^{*}, 3 R^{*}\right)-2,3$-Dibromo-2methylbutan-1-ol $\left(R^{*}, R^{*}\right)$-10. Ellipsoids are shown at 50\% probability. $n$-Hexyl chains are omitted for clarity.

\section{X-ray Co-Crystal Structure as Determined by Single Crystal X-Ray Diffraction: $(M)_{4}$ -} AAC $\supset( \pm)-\left(2 R^{*}, 3 R^{*}\right)-2,3-D i b r o m o-2-m e t h y l b u t a n-1-o l ~\left(R^{*}, R^{*}\right)-10$ : CCDC-1914859

Single crystals were grown following the general crystallization protocol. All protons were detected crystallographically. The H-bonding array shows a counter-clockwise orientation. The guest $\left(R^{*}, R^{*}\right)$-10 is disordered over two positions with major and minor occupancies of $65 \%$ and $35 \%$. The different occupancy of the guest molecule corresponds to the two enantiomeric conformers depicted below. 


Empirical formula
Formula weight
Temperature/K
Crystal system
Space group
$\mathrm{a} / \AA$
$\mathrm{b} / \AA$
$\mathrm{c} / \AA$
$\alpha /{ }^{\circ}$
$\beta /{ }^{\circ}$
$\gamma /{ }^{\circ}$
Volume/ $\AA^{3}$
$\mathrm{Z}$
$\rho_{\text {calc }} / \mathrm{cm}^{3}$
$\mu / \mathrm{mm}^{-1}$
$\mathrm{~F}(000)$
Crystal size $/ \mathrm{mm}^{3}$
Radiation
$2 \Theta$ range for data collection $/{ }^{\circ}$
Index ranges
Reflections collected
Independent reflections
Data/restraints $/$ parameters
Goodness-of-fit on $\mathrm{F}^{2}$
Final $R$ indexes $[\mathrm{I}>=2 \sigma(\mathrm{I})]$
Final $R$ indexes $[$ all data $]$
Flack parameter

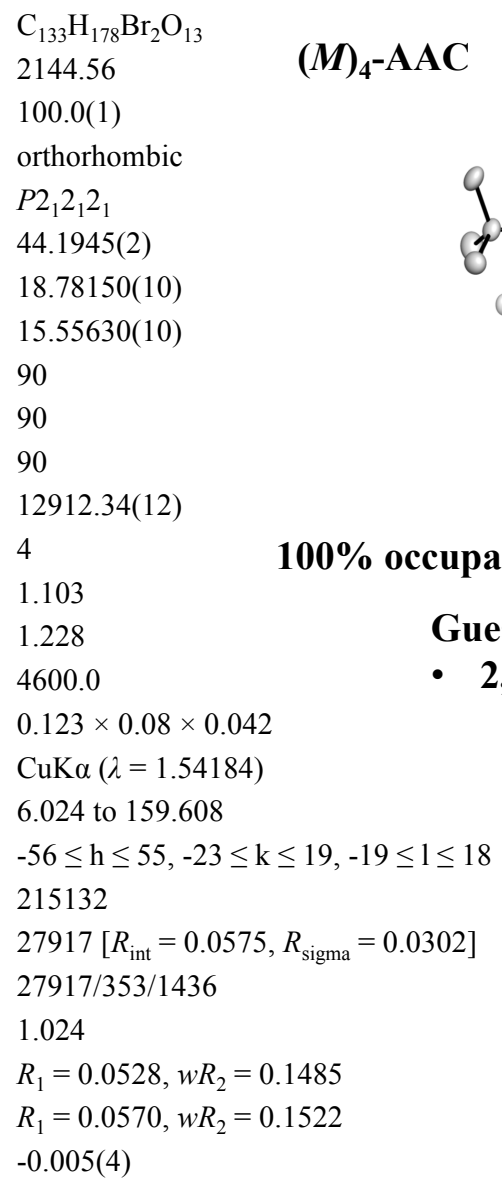

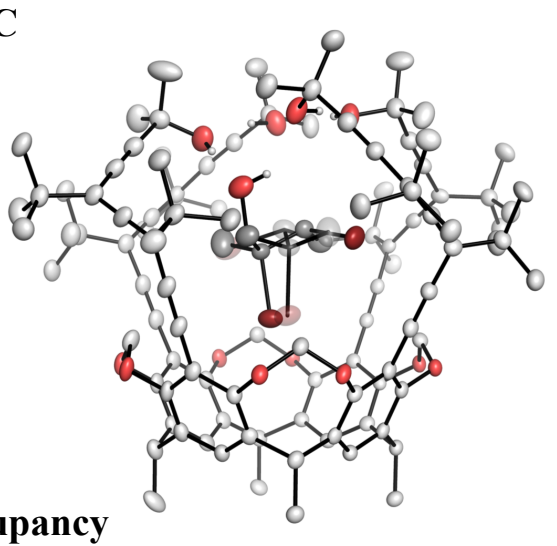

Guest in cavity:

- 2,3-dibromo-2-methylbutan-1-ol<smiles>C[C@H](Br)[C@](C)(Br)CO</smiles>

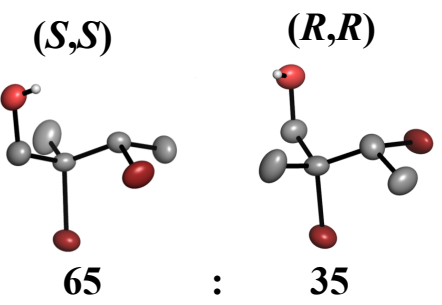

Figure S102. Crystallographic data and ORTEP representation of $(M)_{4}$-AAC $\supset( \pm)-\left(2 R^{*}, 3 R^{*}\right)-2,3$-Dibromo-2methylbutan-1-ol $\left(R^{*}, R^{*}\right)$-10. Ellipsoids are shown at 50\% probability. $n$-Hexyl chains are omitted for clarity. 


\section{S12. NMR Spectra of $(P)_{4}$-Configured AAC and Guest Compounds}

$400 \mathrm{MHz}$ NMR

$\mathrm{CDCl}_{3}$

$298 \mathrm{~K}$
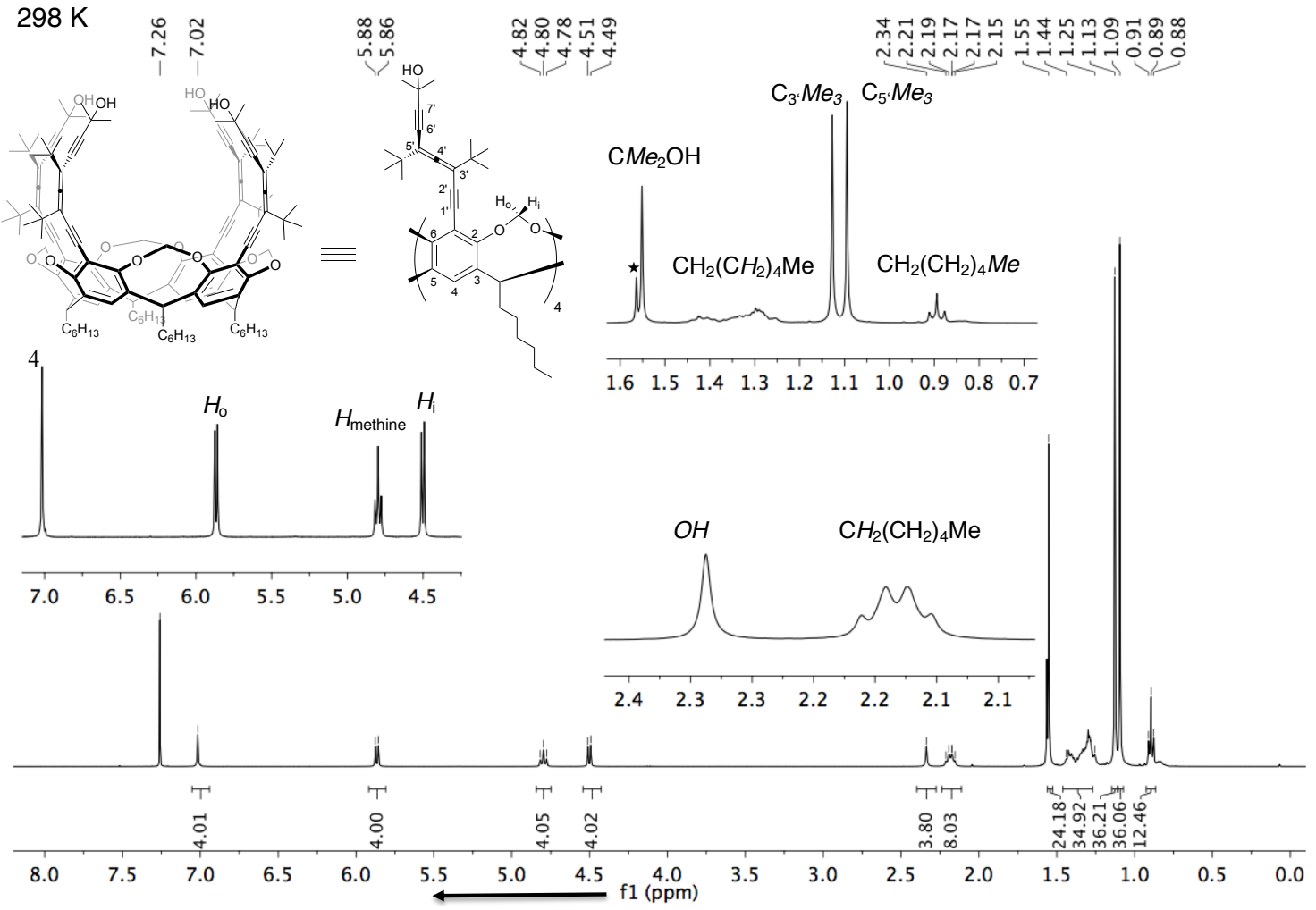

Figure S103. ${ }^{1} \mathrm{H}$ NMR (400 MHz) spectrum of AAC $(P)-1$ in $\mathrm{CDCl}_{3} .{ }^{[1]}$

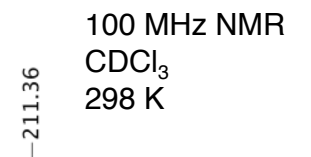

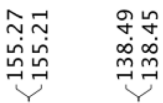

๙ิ

ᄀ
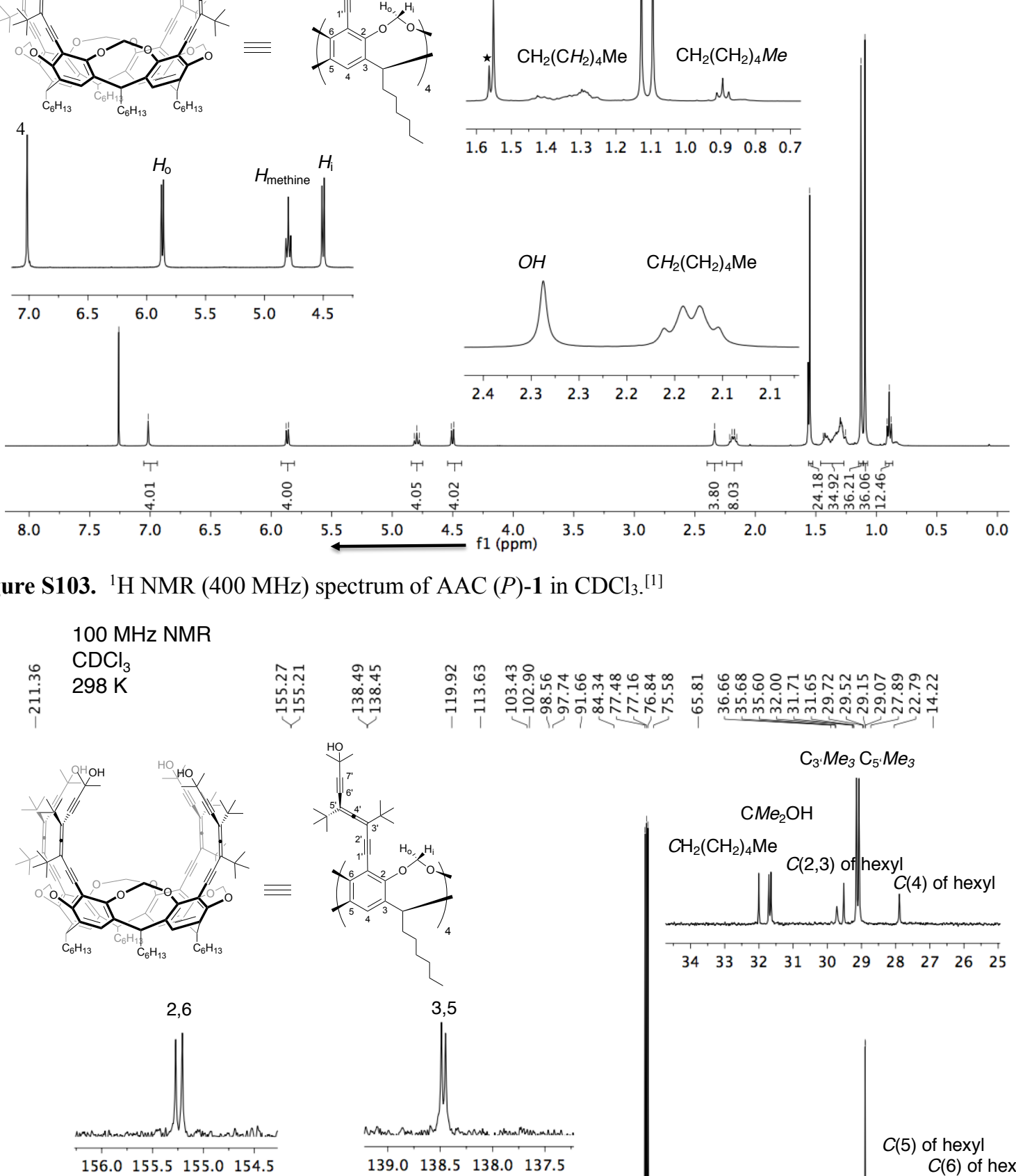
$400 \mathrm{MHz}$ NMR

$\mathrm{CDCl}_{3}$

$298 \mathrm{~K}$

$\mathrm{Me}_{\mathrm{OH}}$

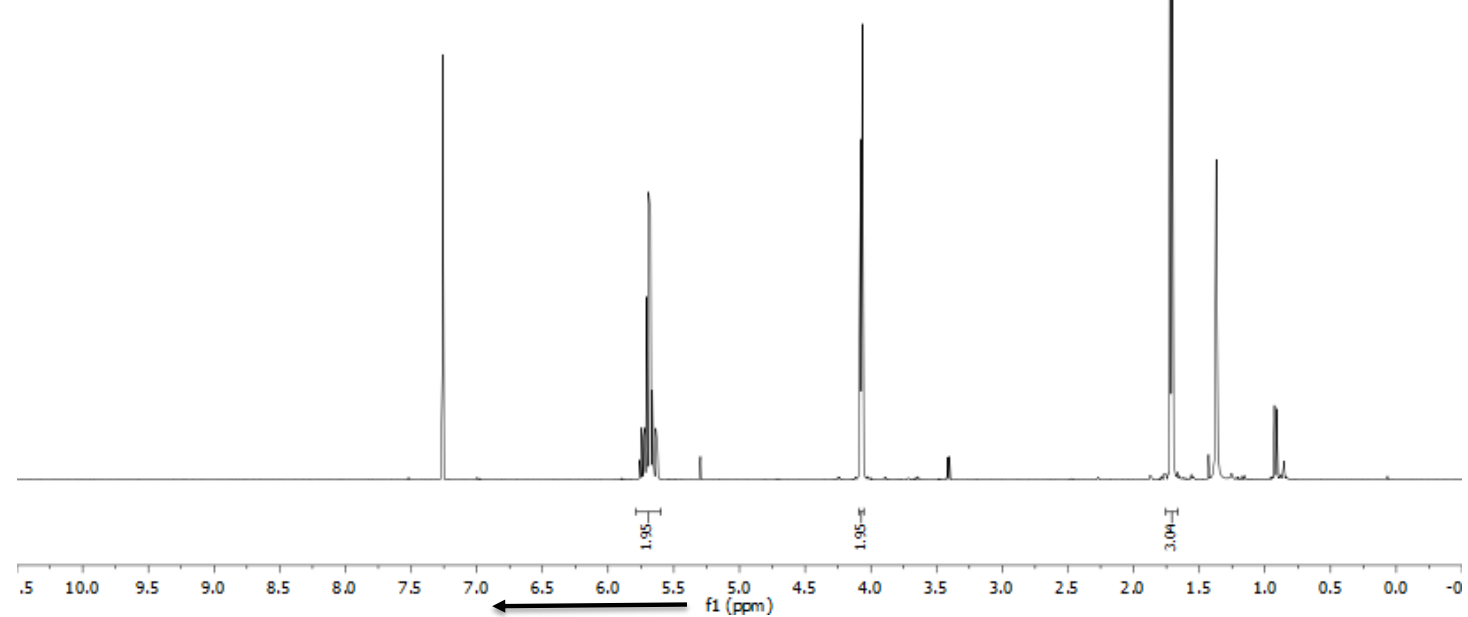

Figure S105. ${ }^{1} \mathrm{H}$ NMR (400 MHz) spectrum of 14 in $\mathrm{CDCl}_{3}$.

$400 \mathrm{MHz}$ NMR

$\mathrm{CDCl}_{3}$

$298 \mathrm{~K}$

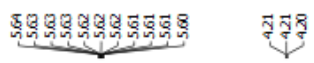

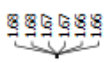

$\stackrel{\mathrm{Me}}{\mathrm{OH}}$

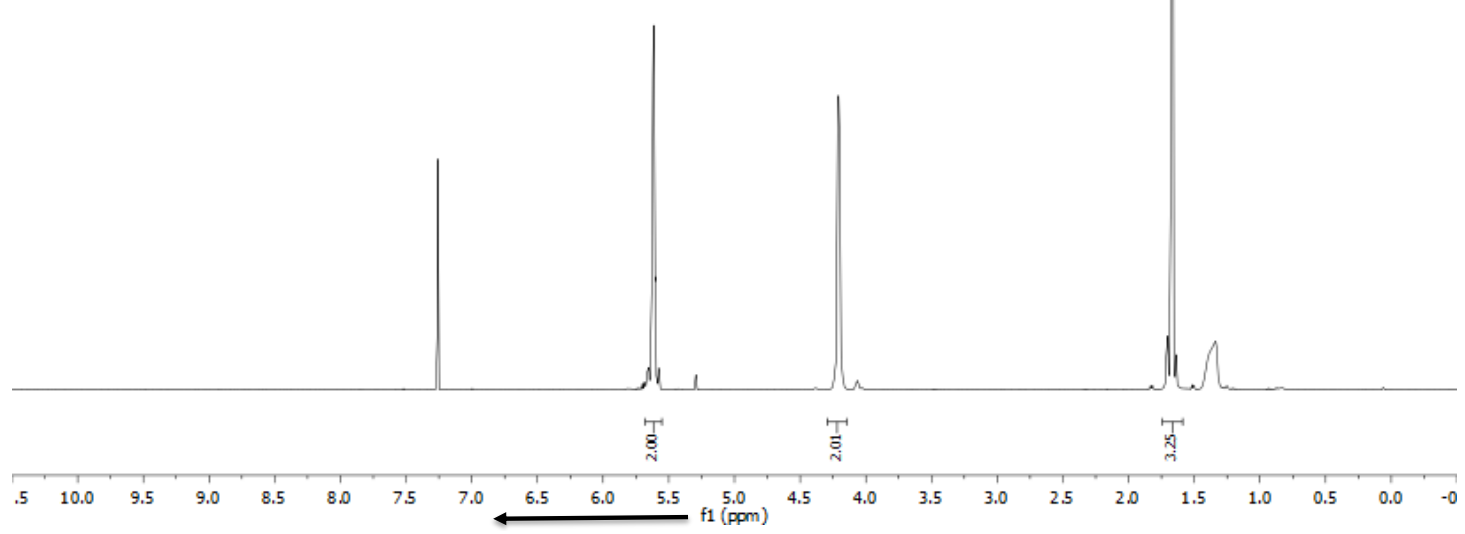

Figure S106. ${ }^{1} \mathrm{H}$ NMR (400 MHz) spectrum of 16 in $\mathrm{CDCl}_{3}$. 
$400 \mathrm{MHz}$ NMR

$\mathrm{CDCl}_{3}$

$298 \mathrm{~K}$

$\mathrm{Me}_{\mathrm{OH}}$

$\mathrm{Me}$

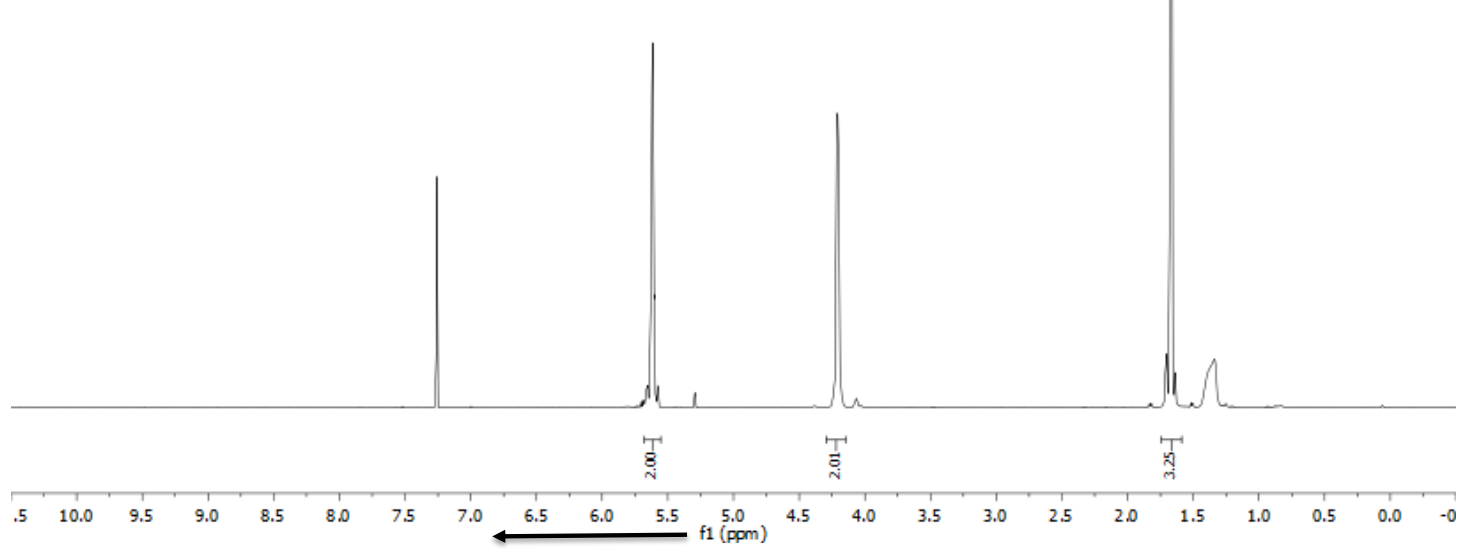

Figure S107. ${ }^{1} \mathrm{H}$ NMR (400 MHz) spectrum of 18 in $\mathrm{CDCl}_{3}$.

$400 \mathrm{MHz}$ NMR

$\mathrm{CDCl}_{3}$

$298 \mathrm{~K}$

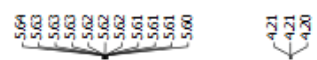

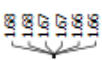

$\underbrace{\text { Me }}_{\text {Me }}$

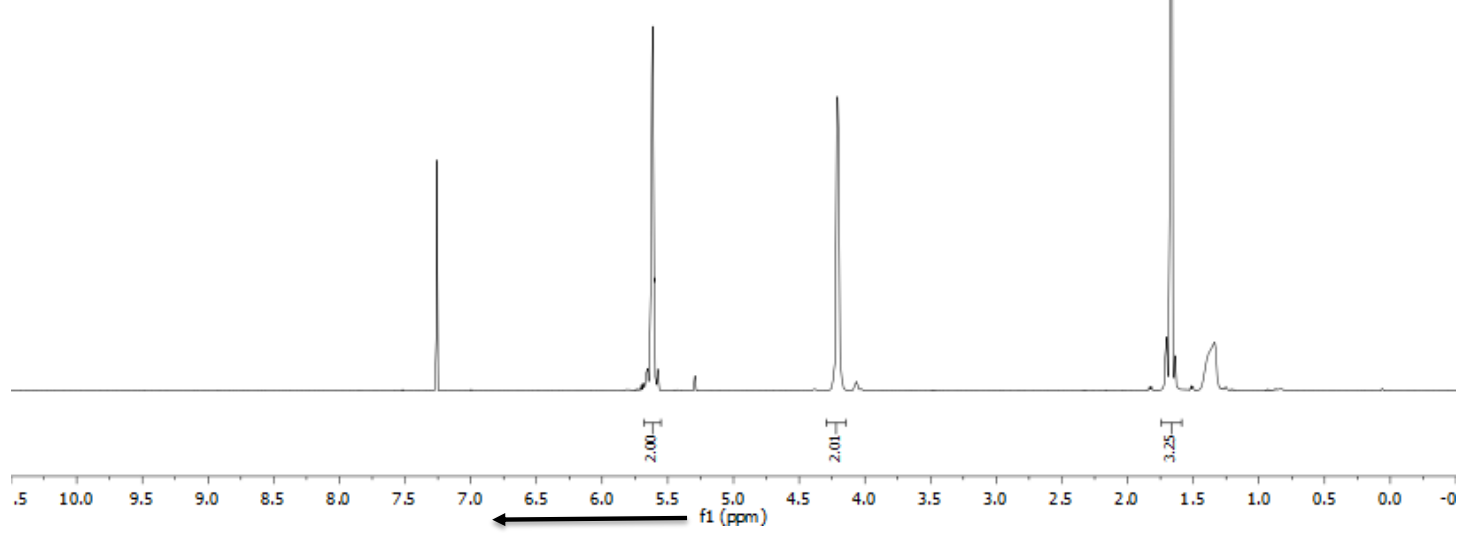

Figure S108. ${ }^{1} \mathrm{H}$ NMR (400 MHz) spectrum of 20 in $\mathrm{CDCl}_{3}$. 
$400 \mathrm{MHz}$ NMR

$\mathrm{CDCl}_{3}$

$298 \mathrm{~K}$<smiles>C[C](Br)[C@H](Br)CO</smiles>

$( \pm)$

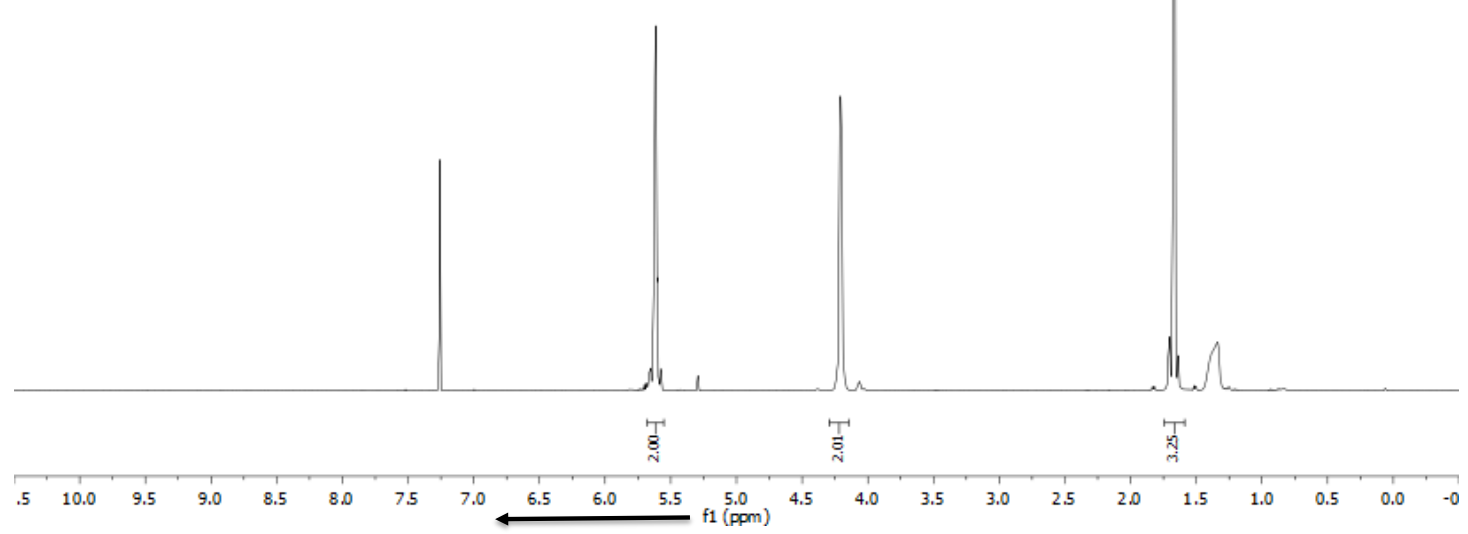

Figure S109. ${ }^{1} \mathrm{H}$ NMR (400 MHz) spectrum of $\left(R^{*}, R^{*}\right)-8$ in $\mathrm{CDCl}_{3}$.

$100 \mathrm{MHz}$ NMR

$\mathrm{CDCl}_{3}$

$298 \mathrm{~K}$<smiles>C[C@@H](Br)[C@H](Br)CO</smiles>

$( \pm)$

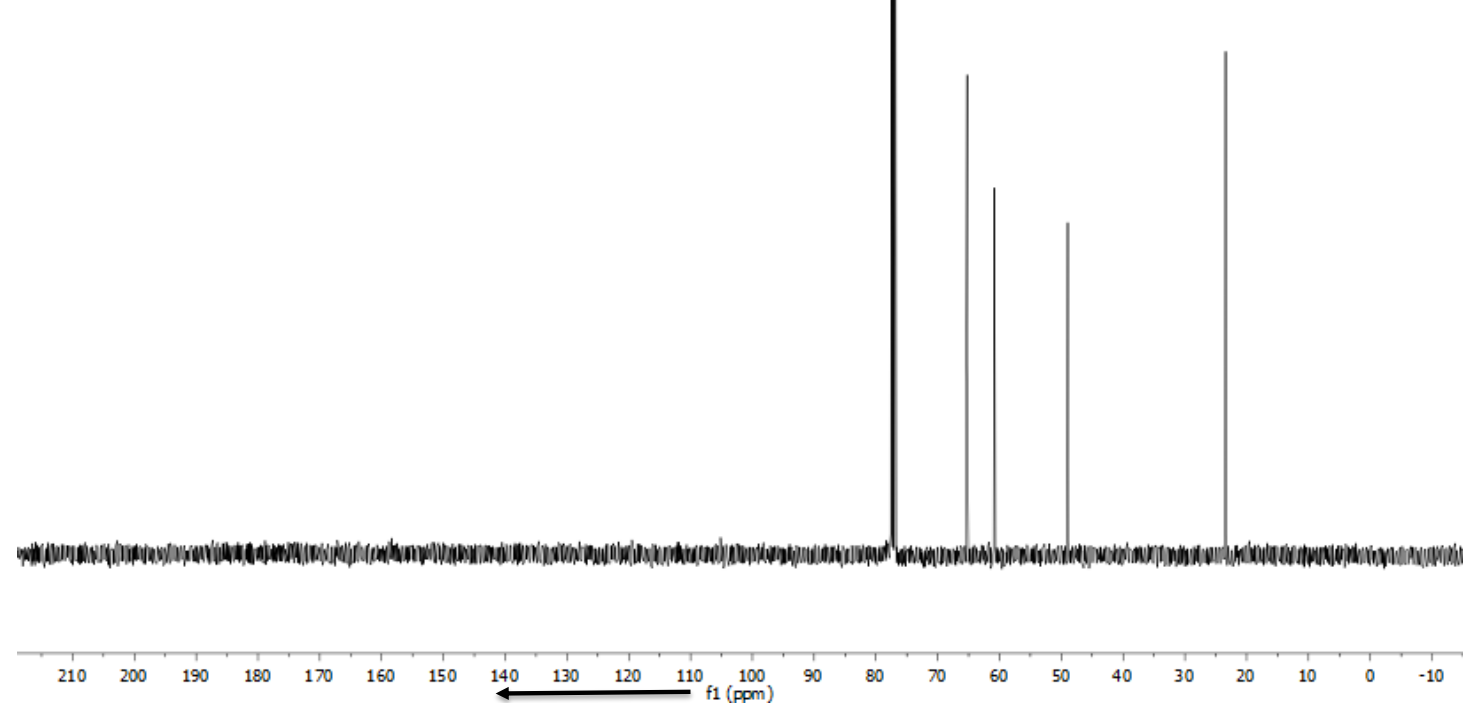

Figure S110. ${ }^{13} \mathrm{C}$ NMR (400 MHz) spectrum of $\left(R^{*}, R^{*}\right)-8$ in $\mathrm{CDCl}_{3}$. 
$400 \mathrm{MHz}$ NMR

$\mathrm{CDCl}_{3}$

$298 \mathrm{~K}$<smiles>C[C@@H](Br)[C@H](Br)CO</smiles>

$( \pm)$

Wh

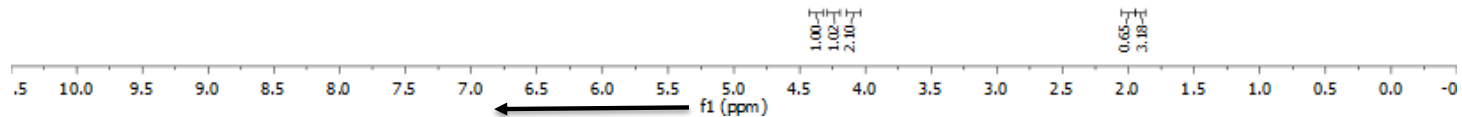

Figure S111. ${ }^{1} \mathrm{H}$ NMR (400 MHz) spectrum of $\left(R^{*}, S^{*}\right)-8$ in $\mathrm{CDCl}_{3}$.

$100 \mathrm{MHz}$ NMR

$\mathrm{CDCl}_{3}$

$298 \mathrm{~K}$<smiles>C[C@@H](Br)[C@H](Br)CO</smiles>

$( \pm)$

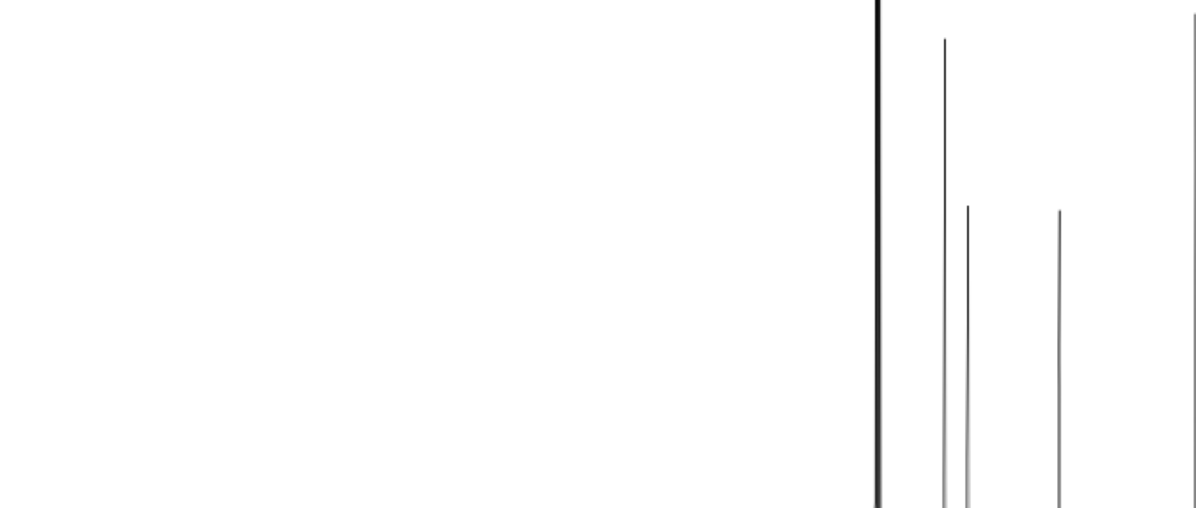

Figure S112. ${ }^{13} \mathrm{C}$ NMR $(400 \mathrm{MHz})$ spectrum of $\left(R^{*}, S^{*}\right)$-8 in $\mathrm{CDCl}_{3}$. 
$400 \mathrm{MHz}$ NMR

$\mathrm{CDCl}_{3}$

$298 \mathrm{~K}$

$\overbrace{\mathrm{Cl}}^{\mathrm{Cl}} \mathrm{OH}$

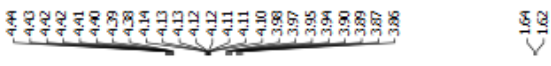

$( \pm)$

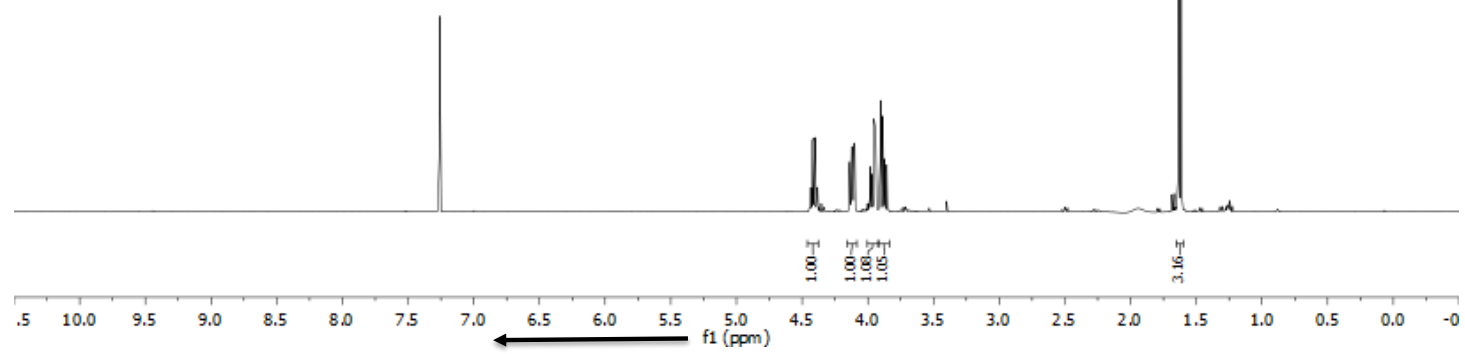

Figure S113. ${ }^{1} \mathrm{H}$ NMR (400 MHz) spectrum of $\left(R^{*}, R^{*}\right)-7$ in $\mathrm{CDCl}_{3}$.

$100 \mathrm{MHz} N M R$
$\mathrm{CDCl}_{3}$
$298 \mathrm{~K}$

$( \pm)$
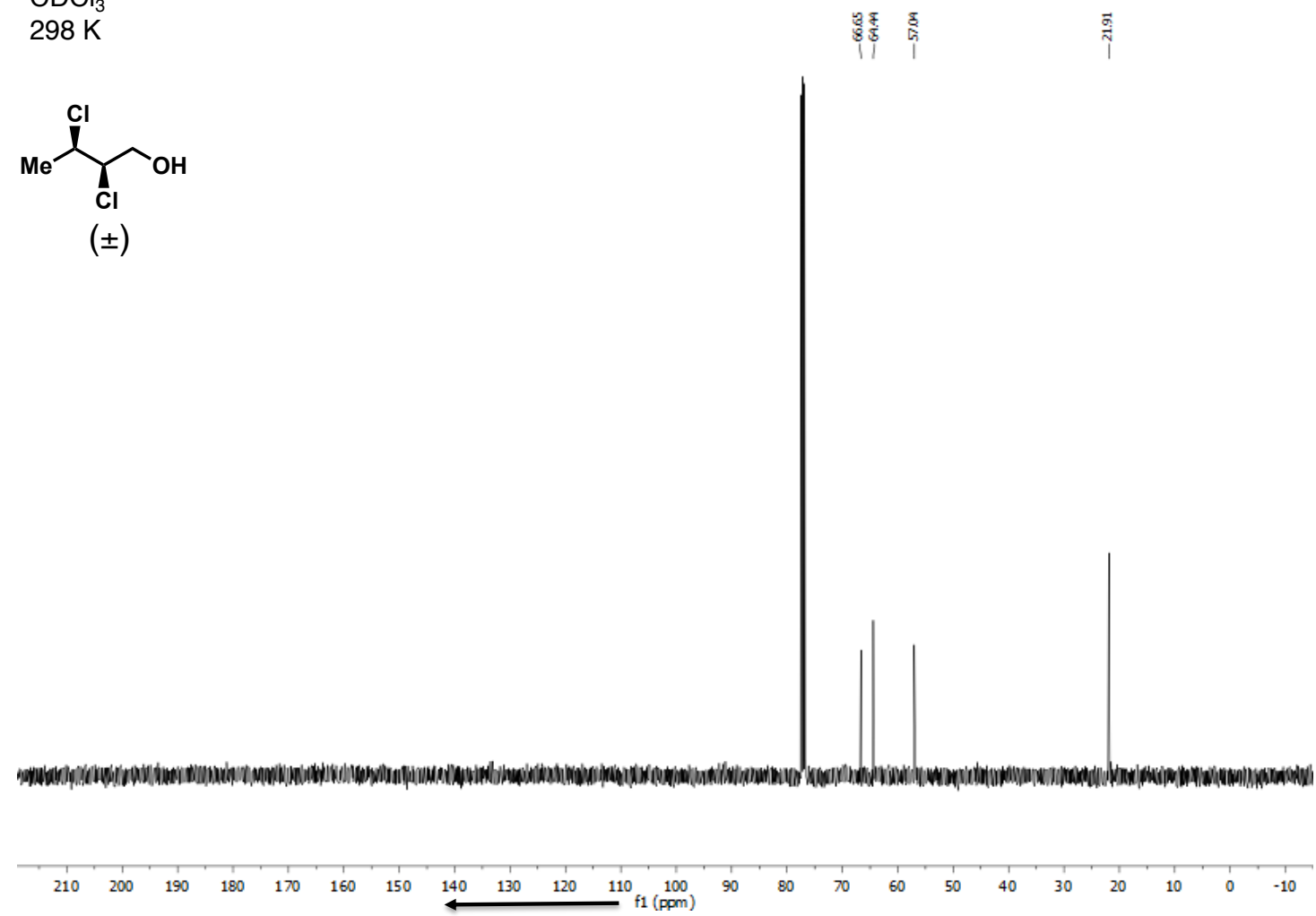

Figure S114. ${ }^{13} \mathrm{C}$ NMR (400 MHz) spectrum of $\left(R^{*}, R^{*}\right)-7$ in $\mathrm{CDCl}_{3}$. 
$400 \mathrm{MHz}$ NMR

$\mathrm{CDCl}_{3}$

$298 \mathrm{~K}$

\section{춘}<smiles>[Y6]C(Cl)[C](Cl)CO</smiles>

$( \pm)$

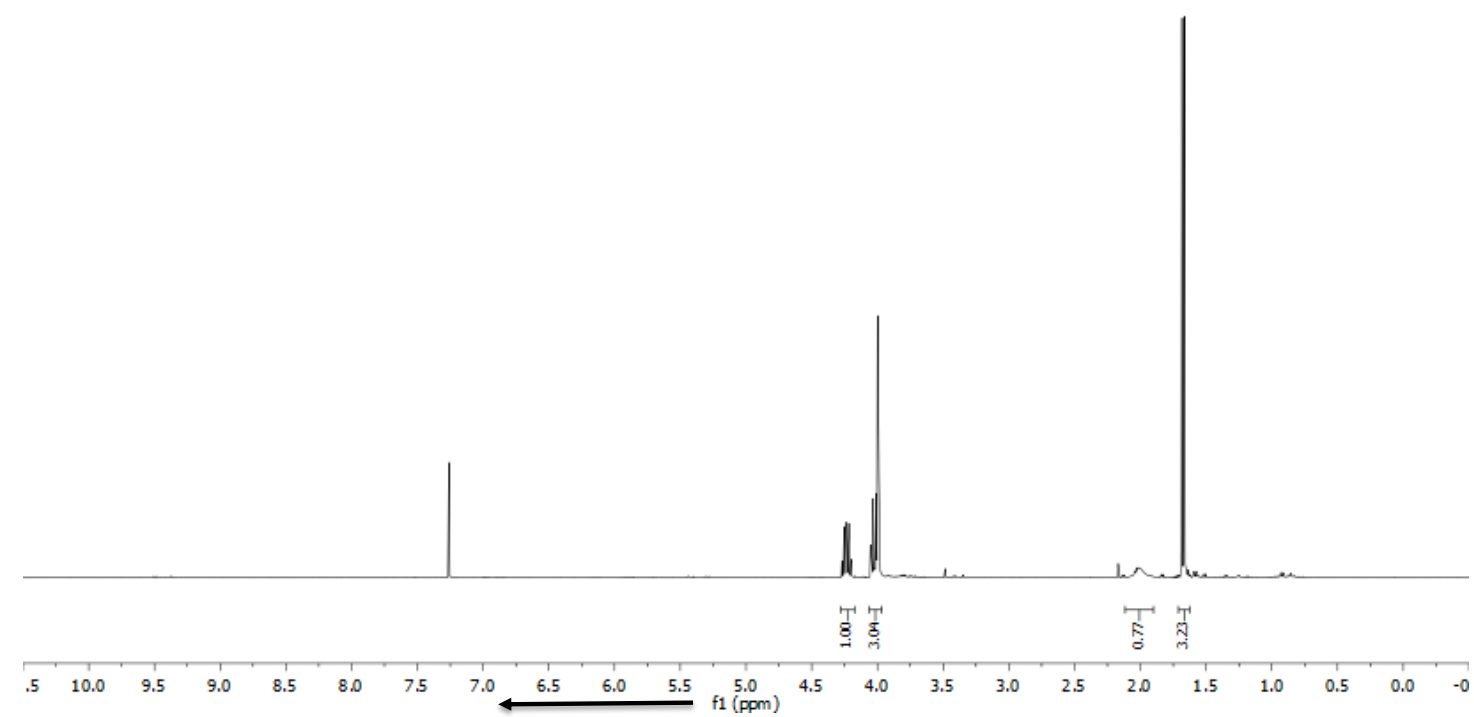

Figure S115. ${ }^{1} \mathrm{H}$ NMR (400 MHz) spectrum of $\left(R^{*}, S^{*}\right)-7$ in $\mathrm{CDCl}_{3}$.

$100 \mathrm{MHz}^{\mathrm{NMR}}$
$\mathrm{CDCl}_{3}$
$298 \mathrm{~K}$

$( \pm)$

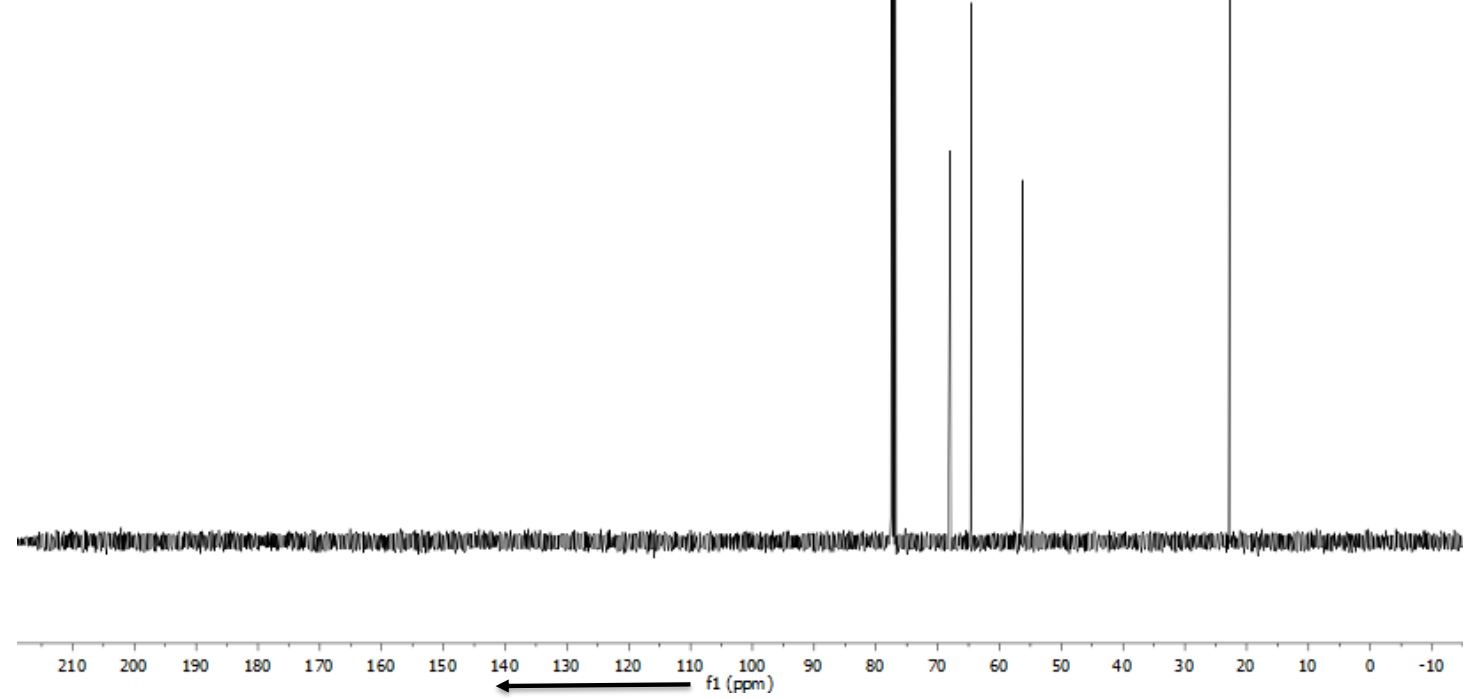

Figure S116. ${ }^{13} \mathrm{C}$ NMR (400 MHz) spectrum of $\left(R^{*}, S^{*}\right)-7$ in $\mathrm{CDCl}_{3}$. 
$400 \mathrm{MHz}$ NMR

$\mathrm{CDCl}_{3}$

$298 \mathrm{~K}$

$\overbrace{B r}^{\mathrm{Br}} \mathrm{OH}$

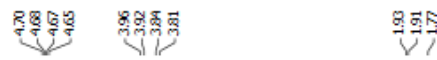

$( \pm)$

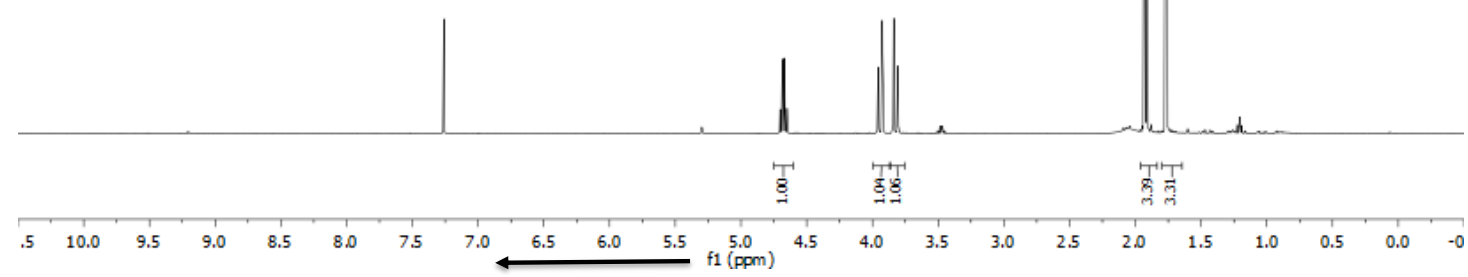

Figure S117. ${ }^{1} \mathrm{H}$ NMR $(400 \mathrm{MHz})$ spectrum of $\left(R^{*}, S^{*}\right)-\mathbf{1 0}$ in $\mathrm{CDCl}_{3}$.

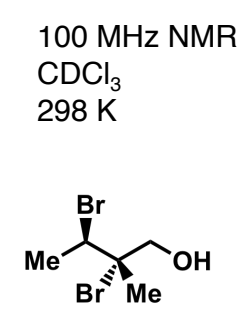

$( \pm)$
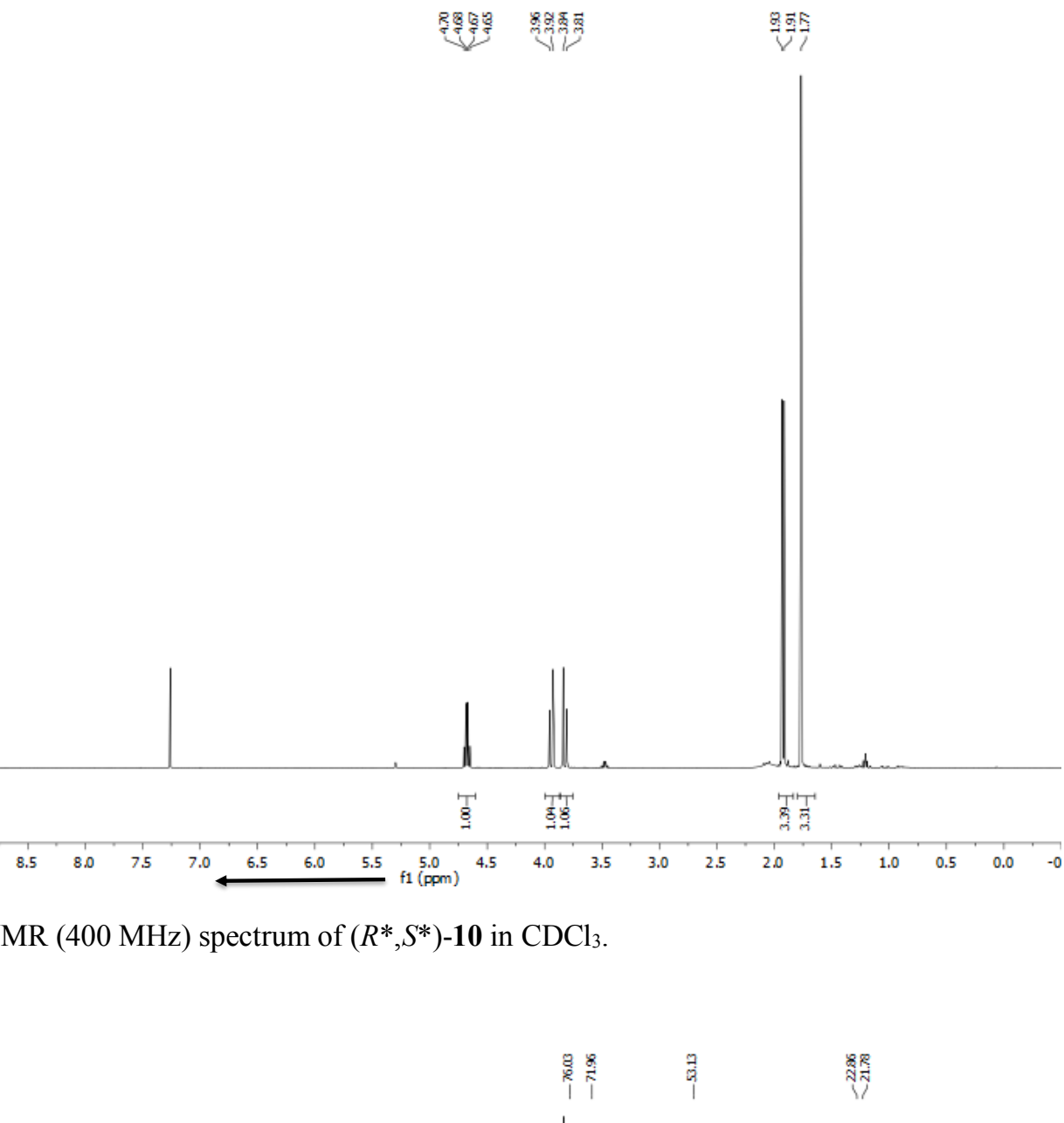

$100 \mathrm{MHz}$ NMR

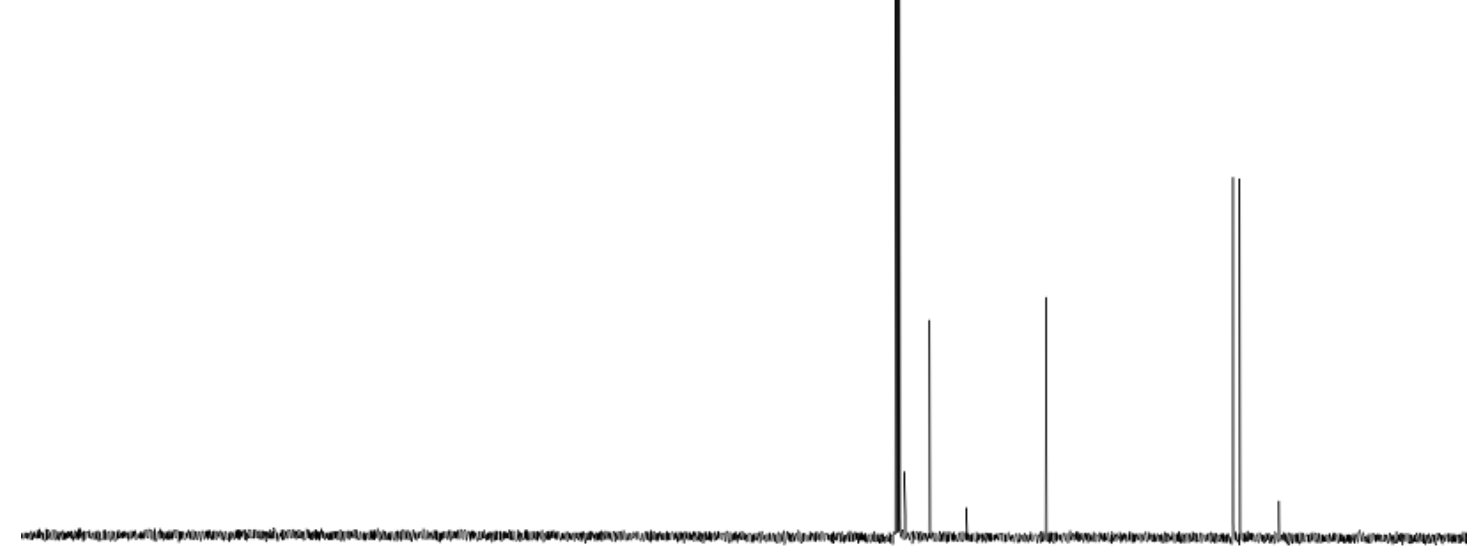

$\begin{array}{llllllllllllllllllllllllllllllllll}210 & 200 & 190 & 180 & 170 & 160 & 150 & 140 & 130 & 120 & 110 & 100 & 90 & 80 & 70 & 60 & 50 & 40 & 30 & 20 & 10 & 0 & -10\end{array}$

Figure S118. ${ }^{13} \mathrm{C}$ NMR $(400 \mathrm{MHz})$ spectrum of $\left(R^{*}, S^{*}\right)-\mathbf{1 0}$ in $\mathrm{CDCl}_{3}$. 
$400 \mathrm{MHz}$ NMR

$\mathrm{CDCl}_{3}$

$298 \mathrm{~K}$<smiles>OCC(Br)(Br)[CH]Br</smiles>

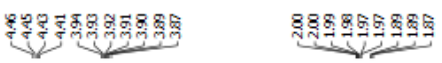

$( \pm)$

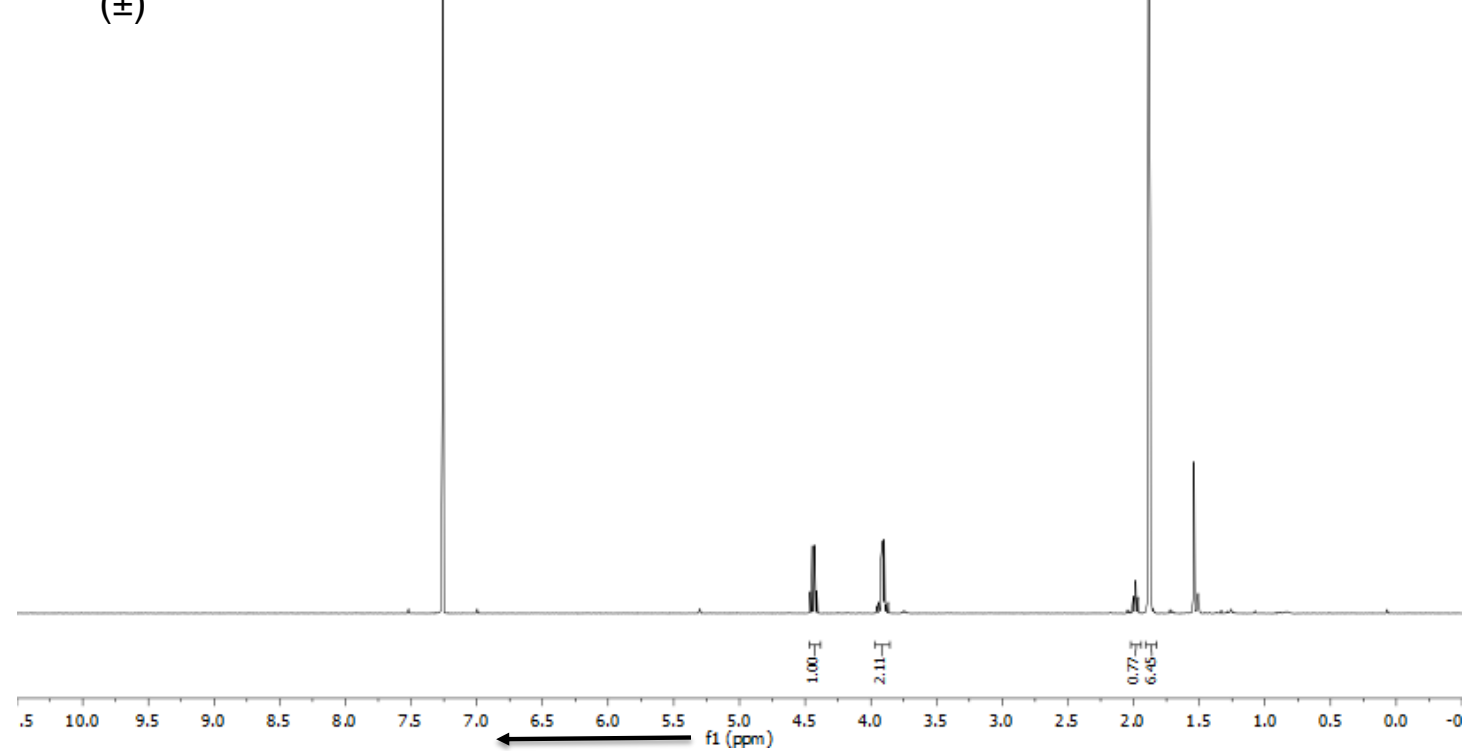

Figure S119. ${ }^{1} \mathrm{H} \mathrm{NMR}(400 \mathrm{MHz})$ spectrum of $\left(R^{*}, R^{*}\right)-10$ in $\mathrm{CDCl}_{3}$.

$100 \mathrm{MHz} N M R$
$\mathrm{CDCl}_{3}$
$298 \mathrm{~K}$

$( \pm)$

$( \pm$

.


$400 \mathrm{MHz}$ NMR

$\mathrm{CDCl}_{3}$

$298 \mathrm{~K}$

$\overbrace{M e}^{M e}{ }_{M e}^{O}$

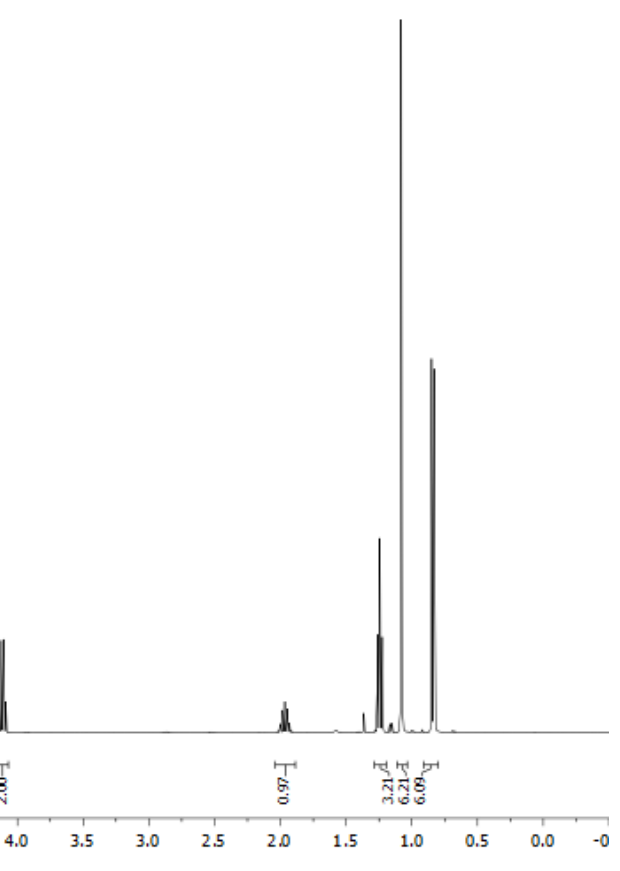

Figure S121. ${ }^{1} \mathrm{H}$ NMR (400 MHz) spectrum of 23 in $\mathrm{CDCl}_{3}$.

$100 \mathrm{MHz}$ NMR

$\mathrm{CDCl}_{3}$

$298 \mathrm{~K}$
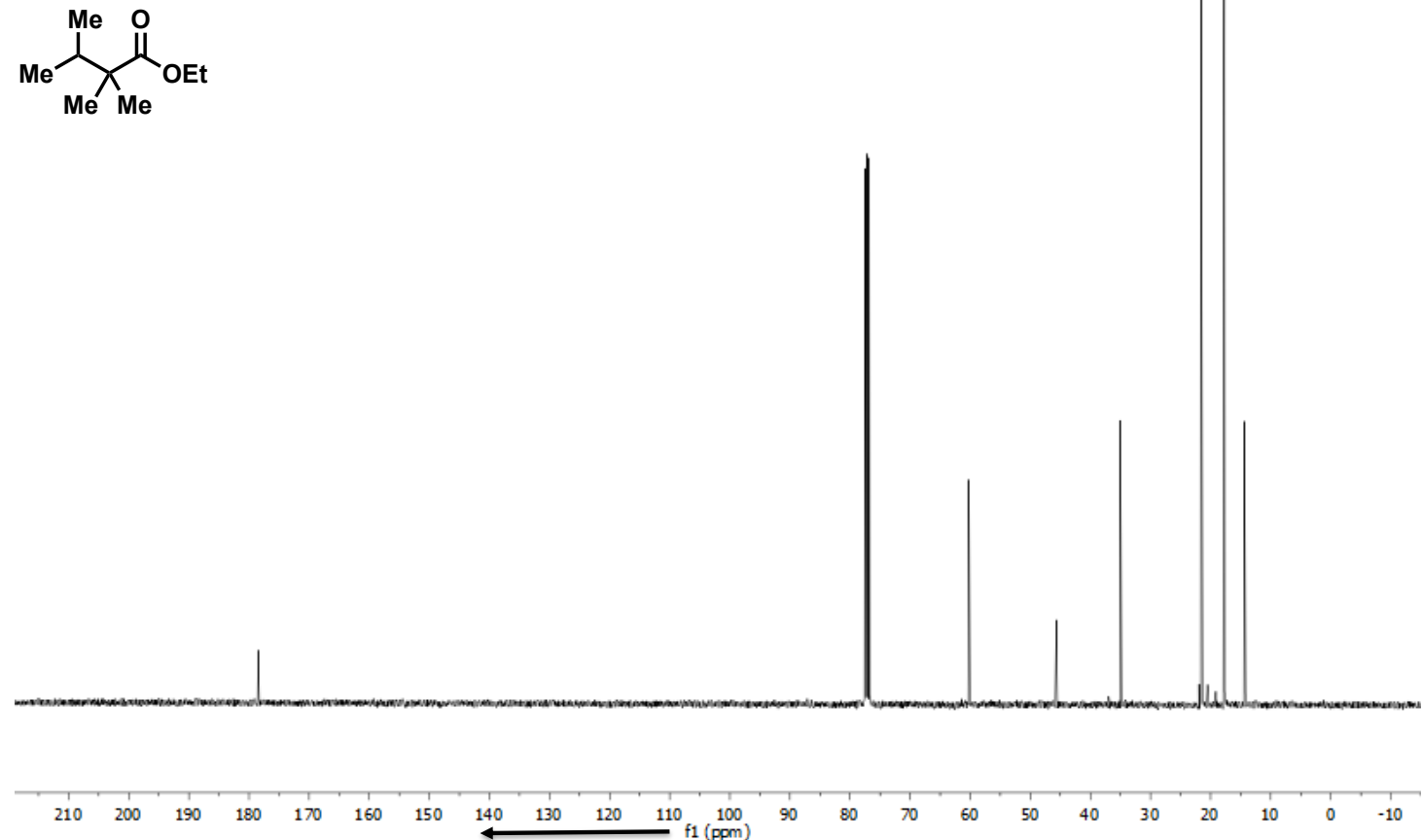

Figure S122. ${ }^{13} \mathrm{C}$ NMR (400 MHz) spectrum of 23 in $\mathrm{CDCl}_{3}$. 
$400 \mathrm{MHz}$ NMR

$\mathrm{CDCl}_{3}$

$298 \mathrm{~K}$

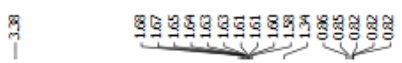

$\overbrace{M e}^{M e} \mathrm{OH}$

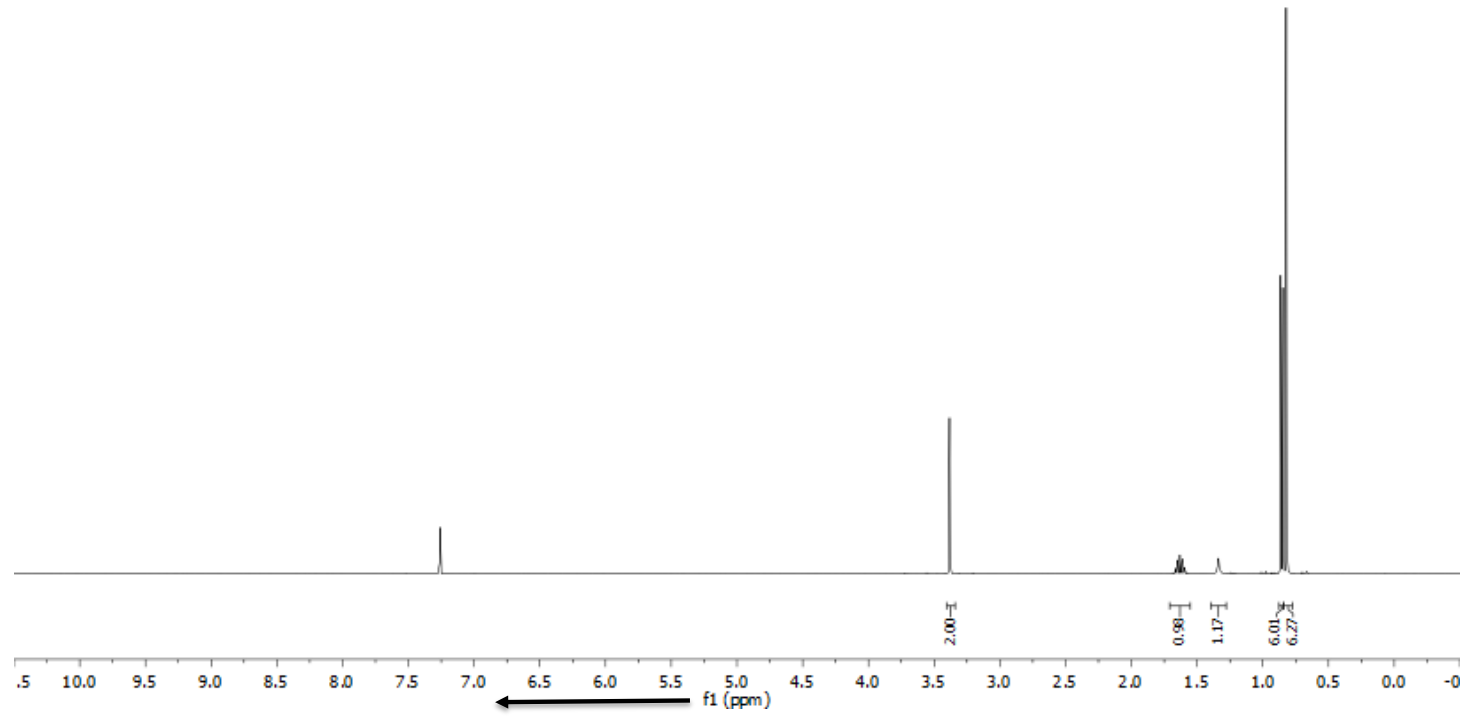

Figure S123. ${ }^{1} \mathrm{H}$ NMR (400 MHz) spectrum of 9 in $\mathrm{CDCl}_{3}$.
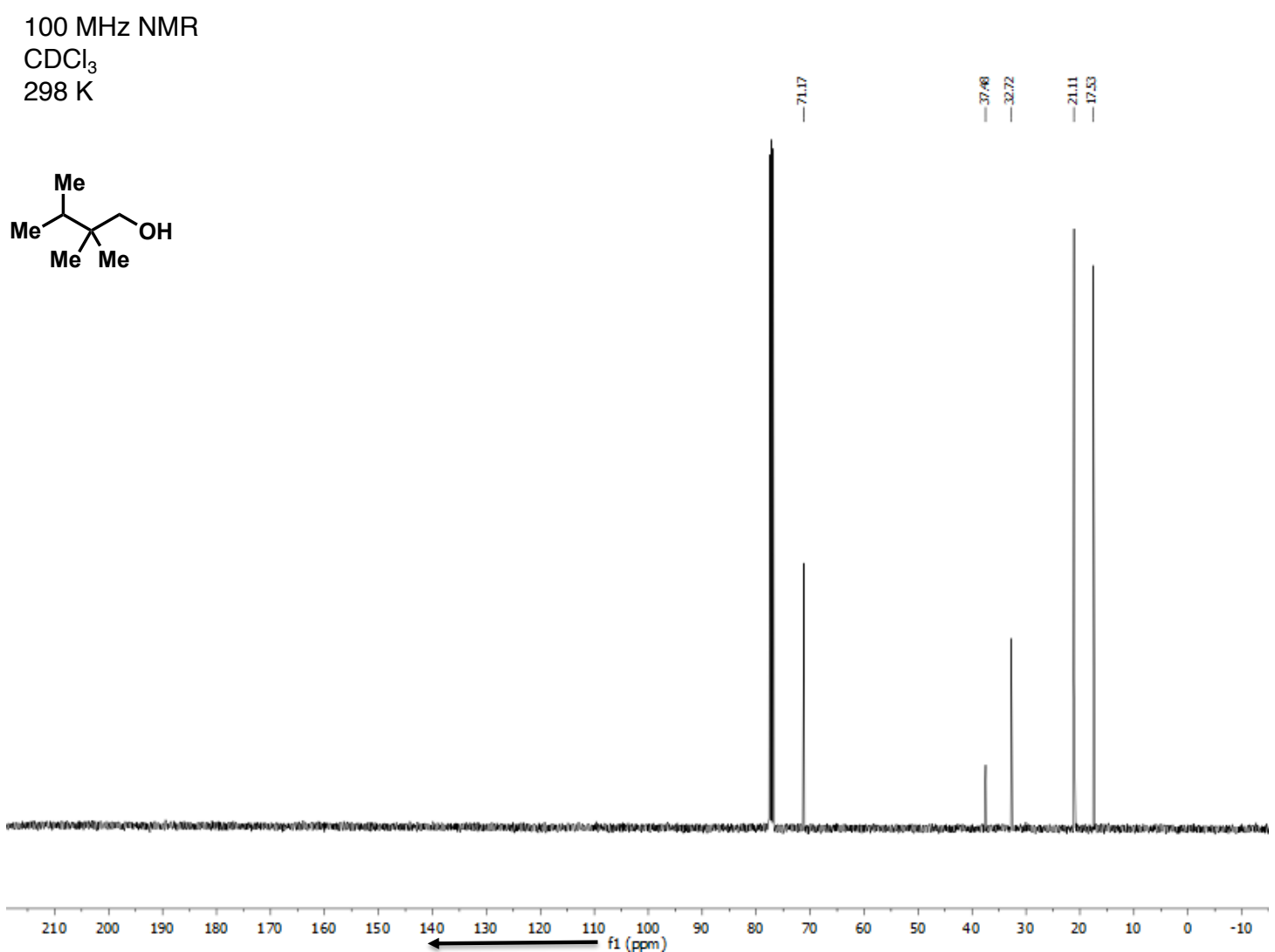

Figure S124. ${ }^{13} \mathrm{C}$ NMR (400 MHz) spectrum of 9 in $\mathrm{CDCl}_{3}$. 
$400 \mathrm{MHz}$ NMR

$\mathrm{CDCl}_{3}$

$298 \mathrm{~K}$<smiles>OC[C@@H](Br)[C@H](Br)C(F)(F)F</smiles>

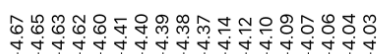

$( \pm)$

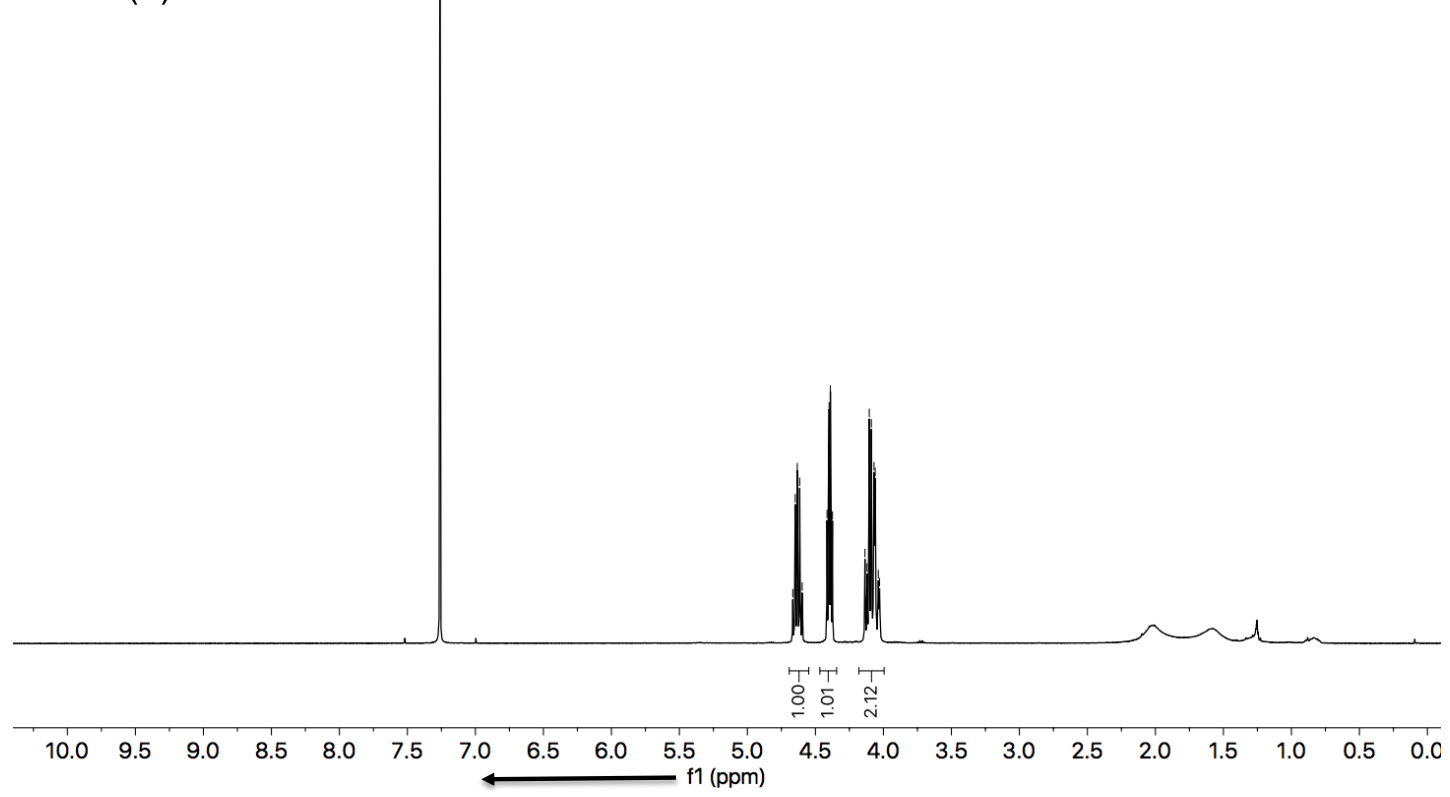

Figure S125. ${ }^{1} \mathrm{H}$ NMR $(400 \mathrm{MHz})$ spectrum of $\left(R^{*}, S^{*}\right)-\mathbf{1 1}$ in $\mathrm{CDCl}_{3}$.

$377 \mathrm{MHz}$ NMR

$\mathrm{CDCl}_{3}$

$298 \mathrm{~K}$<smiles>OC[C@H](Br)[C@H](Br)C(F)(F)F</smiles>

$( \pm)$

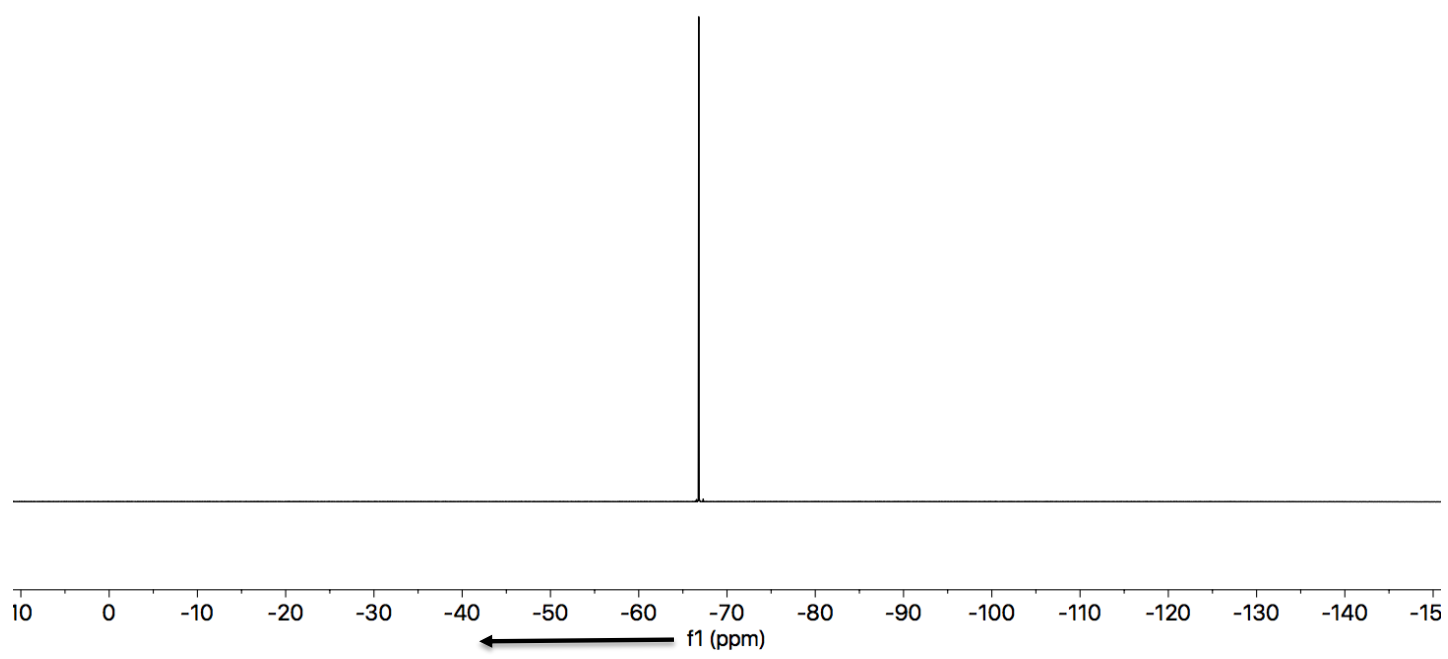

Figure S126. ${ }^{19} \mathrm{~F}$ NMR $(377 \mathrm{MHz})$ spectrum of $\left(R^{*}, S^{*}\right)-11$ in $\mathrm{CDCl}_{3}$. 
$400 \mathrm{MHz}$ NMR

$\mathrm{CDCl}_{3}$

$298 \mathrm{~K}$

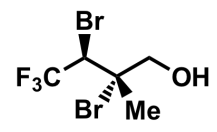

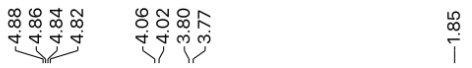

$( \pm)$

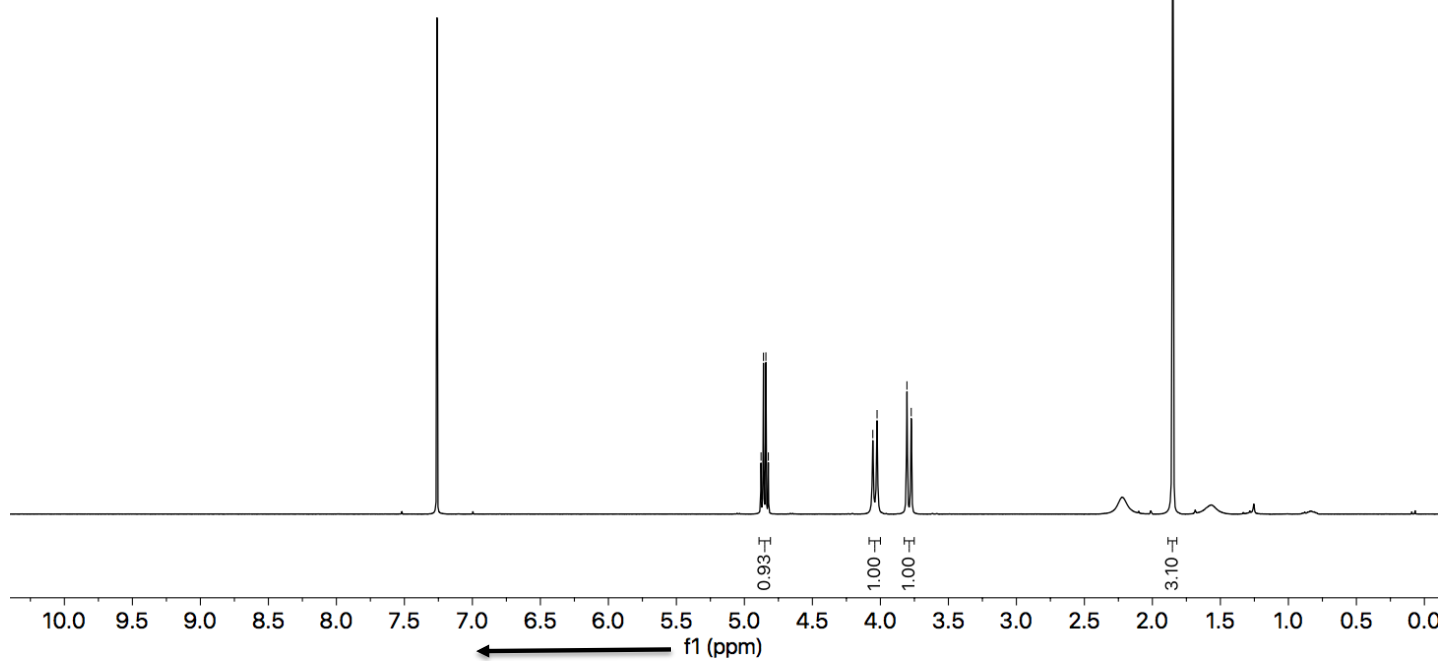

Figure S127. ${ }^{1} \mathrm{H}$ NMR $(400 \mathrm{MHz})$ spectrum of $\left(R^{*}, S^{*}\right)-12$ in $\mathrm{CDCl}_{3}$.

$377 \mathrm{MHz}$ NMR

$\mathrm{CDCl}_{3}$

$298 \mathrm{~K}$

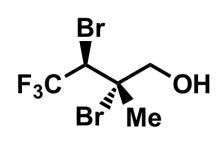

$( \pm)$

$\begin{array}{lllllllllll}-20 & -25 & -30 & -35 & -40 & -45 & -50 & -55 & -60 & -65 & -70\end{array}$

Figure S128. ${ }^{19} \mathrm{~F}$ NMR (377 MHz) spectrum of $\left(R^{*}, S^{*}\right)-12$ in $\mathrm{CDCl}_{3}$. 


\section{Theoretical Section}

\section{S1. Computational Methodology}

We generate the structures for the guest molecules 2-12 with the exhaustive conformer generator $\mathrm{CONFAB}^{[10]}$ implemented in the Open Babel package. ${ }^{[11]}$ All subsequent structure optimizations were carried out with ORCA (version 4.0.1). ${ }^{[12]}$ We applied the PBE functional ${ }^{[13]}$ including empirical dispersion corrections ("D3") with a Becke-Johnson damping function ${ }^{[14]}$ for all structure optimizations. We chose the def2-TZVPP basis set ${ }^{[15]}$ for all calculations and invoked the resolution-of-the-identity density fitting of the Coulomb integrals. For all molecules, we carried out structure optimizations in gas phase and in an implicit continuum solvent simulating acetonitrile ${ }^{[16]}$ (CPCM(Acetonitrile) keyword). The maximum norm of the Cartesian structure gradient was converged to $10^{-3}$ Hartree bohr $^{-1}$ in all cases.

We determined thermal corrections with the standard rigid-rotor-harmonic-oscillator (RRHO) model in the gas phase ${ }^{[17]}$ based on frequencies calculated with PBE-D3(BJ)/def2-TZVPP. Additionally, we carried out DLPNO-CCSD $(\mathrm{T})^{[18]}$ single-point energy evaluations with the ORCA program for the PBE/def2-TZVPP optimized gas-phase structures. We chose a def2TZVPP basis set ${ }^{[15]}$ and appropriate fitting bases. The single-point energies were converged to $10^{-8}$ Hartree (TightSCF).

A calculation of a single-point energy with one method (e.g., DLPNO-CCSD(T)) for a structure optimized with another method (e.g., PBE-D3(BJ)) is denoted by a combination of the two methods with a double slash (e.g., DLPNO-CCSD(T)/PBE-D3(BJ)).

The Cartesian coordinates of all optimized structures (in Ångstrom) are included as Supporting Material, the second line of each file contains the single-point energies obtained for the structure.

\section{S2. Conformational Analysis}

We determined the preferred conformations around the $\mathrm{C}(2)-\mathrm{C}(3)$ bond in the isolated guest molecules where " $\mathrm{C}(2)$ " refers to the second carbon atom in the butan-1-ol backbone and the "C(3)" refers to the third carbon atom (see also Table S41). For each of the $N$ conformers, we evaluate the respective Boltzmann weight $P$ at room temperature $(T=295 \mathrm{~K})$,

$$
P_{i}=\frac{\exp \left(-E_{i} /\left(k_{B} T\right)\right)}{\sum_{j=1}^{N} \exp \left(-E_{j} /\left(k_{B} T\right)\right)}
$$

where $k_{B}$ is the Boltzmann constant and $E_{i}$ the electronic energy of the $i$-th conformation of a molecule (see Table S41). Each structure 2-12 features a different substitution pattern for the 
substituents $R_{1}, R_{2}, R_{3}$, and $R_{4}$ which is also specified in Table S41. For easier readability, we present the results additionally in Figure S129.

Table S41. Boltzmann populations $\mathrm{P}$ at $295 \mathrm{~K}$ for the three groups of conformers shown as Newman projections for 2-12. The Boltzmann populations are based on PBE-D3(BJ)//PBE-D3(BJ) electronic energy differences in the gas phase (mimicking $n$-octane). We also provide Boltzmann populations based on PBE-D3(BJ)-CPCM//PBE$\mathrm{D} 3(\mathrm{BJ})-\mathrm{CPCM}$ energy differences in the condensed phase (acetonitrile) in parenthesis. We specify the rests $\mathrm{R}_{1-4}$ on $\mathrm{C}(3)$ (in the foreground of the Newman projections) and C(2) (in the background of the Newman projections) for each structure.

\begin{tabular}{|c|c|c|c|c|c|}
\hline & $\begin{array}{l}\text { Substituent on } \\
\qquad \mathrm{C}(3)\end{array}$ & $\begin{array}{l}\text { Substituent on } \\
\qquad \mathrm{C}(2)\end{array}$ & (R) & $\overbrace{\mathrm{R}_{2}}^{\mathrm{R}_{3}}$ & $\overbrace{R_{3}}^{\mathrm{R}_{\mathrm{R}_{2}}}$ \\
\hline 2 & $\mathrm{R}_{1}, \mathrm{R}_{2}=\mathrm{H}$ & $\mathrm{R}_{3}=\mathrm{H}, \mathrm{R}_{4}=\mathrm{Me}$ & $0.58(0.62)$ & $0.25(0.62)$ & $0.18(0.13)$ \\
\hline 3 & $\mathrm{R}_{1}, \mathrm{R}_{2}=\mathrm{H}$ & $\mathrm{R}_{3}, \mathrm{R}_{4}=\mathrm{Me}$ & $0.38(0.35)$ & $0.06(0.06)$ & $0.56(0.59)$ \\
\hline 4 & $\mathrm{R}_{1}=\mathrm{H}, \mathrm{R}_{2}=\mathrm{Me}$ & $\mathrm{R}_{3}=\mathrm{H}, \mathrm{R}_{4}=\mathrm{Me}$ & $0.57(0.60)$ & $0.29(0.26)$ & $0.14(0.14)$ \\
\hline 5 & $\mathrm{R}_{1}, \mathrm{R}_{2}=\mathrm{Me}$ & $\mathrm{R}_{3}=\mathrm{H}, \mathrm{R}_{4}=\mathrm{Me}$ & $0.42(0.45)$ & $0.28(0.29)$ & $0.29(0.27)$ \\
\hline 6 & $\mathrm{R}_{1}=\mathrm{H}, \mathrm{R}_{2}=\mathrm{Me}$ & $\mathrm{R}_{3}, \mathrm{R}_{4}=\mathrm{Me}$ & $0.32(0.27)$ & $0.17(0.18)$ & $0.49(0.54)$ \\
\hline$\left(R^{*}, S^{*}\right)-7$ & $\mathrm{R}_{1}=\mathrm{H}, \mathrm{R}_{2}=\mathrm{Cl}$ & $\mathrm{R}_{3}=\mathrm{Cl}, \mathrm{R}_{4}=\mathrm{Me}$ & $0.93(0.65)$ & $0.01(0.09)$ & $0.05(0.27)$ \\
\hline$\left(R^{*}, R^{*}\right)-7$ & $\mathrm{R}_{1}=\mathrm{Cl}, \mathrm{R}_{2}=\mathrm{H}$ & $\mathrm{R}_{3}=\mathrm{Cl}, \mathrm{R}_{4}=\mathrm{Me}$ & $0.31(0.74)$ & $0.65(0.15)$ & $0.04(0.10)$ \\
\hline$\left(R^{*}, S^{*}\right)-8$ & $\mathrm{R}_{1}=\mathrm{H}, \mathrm{R}_{2}=\mathrm{Br}$ & $\mathrm{R}_{3}=\mathrm{Br}, \mathrm{R}_{4}=\mathrm{Me}$ & $0.95(0.74)$ & $0.01(0.07)$ & $0.03(0.19)$ \\
\hline$\left(R^{*}, R^{*}\right)-8$ & $\mathrm{R}_{1}=\mathrm{Cl}, \mathrm{R}_{2}=\mathrm{H}$ & $\mathrm{R}_{3}=\mathrm{Br}, \mathrm{R}_{4}=\mathrm{Me}$ & $0.20(0.68)$ & $0.80(0.29)$ & $0.01(0.04)$ \\
\hline 9 & $\mathrm{R}_{1}, \mathrm{R}_{2}=\mathrm{Me}$ & $\mathrm{R}_{3}, \mathrm{R}_{4}=\mathrm{Me}$ & $0.44(0.45)$ & $0.14(0.15)$ & $0.42(0.40)$ \\
\hline$\left(R^{*}, S^{*}\right)-\mathbf{1 0}$ & $\mathrm{R}_{1}=\mathrm{Me}, \mathrm{R}_{2}=\mathrm{Br}$ & $\mathrm{R}_{3}=\mathrm{Br}, \mathrm{R}_{4}=\mathrm{Me}$ & $0.99(0.94)$ & $0.00(0.0)$ & $0.01(0.06)$ \\
\hline$\left(R^{*}, R^{*}\right)-10$ & $\mathrm{R}_{1}=\mathrm{Br}, \mathrm{R}_{2}=\mathrm{Me}$ & $\mathrm{R}_{3}=\mathrm{Br}, \mathrm{R}_{4}=\mathrm{Me}$ & $0.16(0.57)$ & $0.78(0.26)$ & $0.06(0.16)$ \\
\hline 11 & $\mathrm{R}_{1}=\mathrm{H}, \mathrm{R}_{2}=\mathrm{Br}$ & $\mathrm{R}_{3}=\mathrm{Br}, \mathrm{R}_{4}=\mathrm{CF}_{3}$ & $0.83(0.82)$ & $0.16(0.11)$ & $0.01(0.07)$ \\
\hline$\left(R^{*}, S^{*}\right)-12$ & $\mathrm{R}_{1}=\mathrm{Me}, \mathrm{R}_{2}=\mathrm{Br}$ & $\mathrm{R}_{3}=\mathrm{Br}, \mathrm{R}_{4}=\mathrm{CF}_{3}$ & $0.97(0.93)$ & $0.01(0.03)$ & $0.01(0.04)$ \\
\hline
\end{tabular}

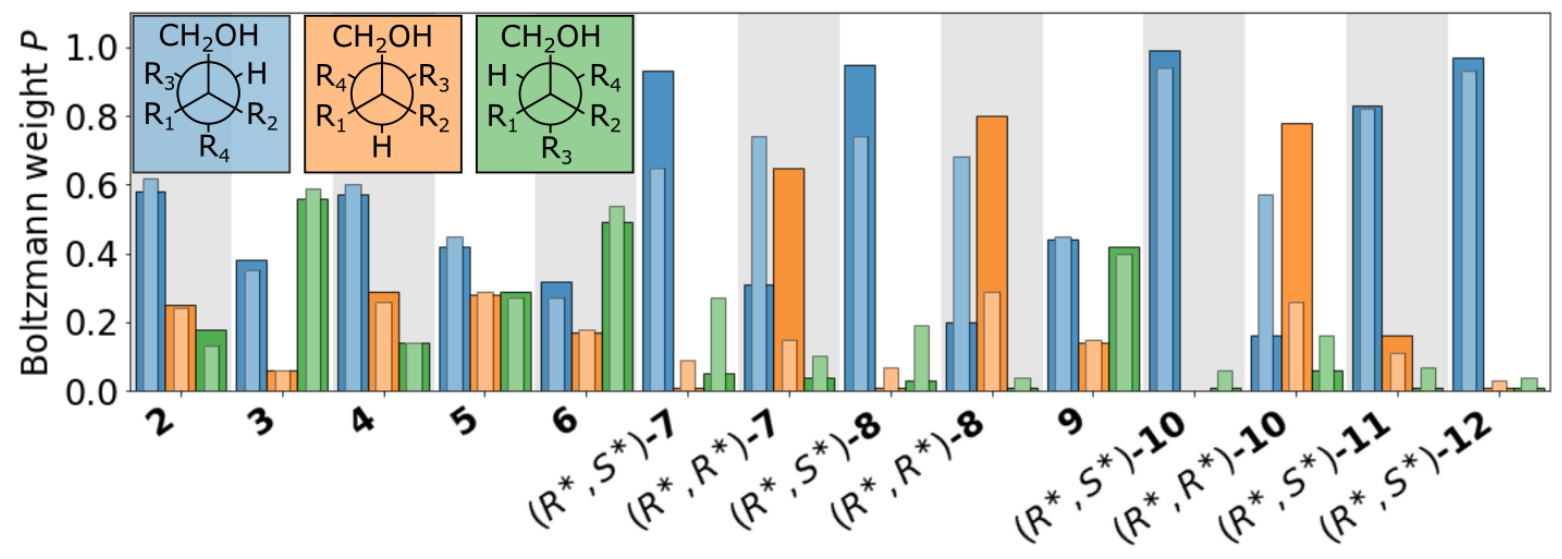

Figure S129. Boltzmann weights $P$ at $295 \mathrm{~K}$ for three conformers displayed as Newman projections (colored blue, orange, and green) for 2-12 based on PBE-D3(BJ)//PBE-D3(BJ) electronic energy differences in the gas phase (solid, dark colored bars) and based on PBE-D3(BJ)-CPCM//PBE-D3(BJ)-CPCM electronic energy 
differences in the condensed phase (translucent, light colored bars). The rests $\mathrm{R}_{1-4}$ for $\mathbf{2}-\mathbf{1 2}$ are specified in Table S41.

The structures which do not feature halogen substituents $(\mathbf{2}, \mathbf{3}, \mathbf{4}, \mathbf{5}, \mathbf{6}, \mathbf{9})$ preferentially adopt a conformation in which the largest substituents on the $\mathrm{C}(2)$ and $\mathrm{C}(3)$ are anti-periplanar to each other (see Table S41). The anti-periplanar conformation is also the conformation which is experimentally observed for $\mathbf{5}$ encapsulated in the host. The encapsulated alcohol $\mathbf{9}$ adopts an anti-periplanar and a gauche structure of the terminal methyl groups relative to the $\mathrm{CH}_{2} \mathrm{OH}$ group. This conformation is also the preferred conformation of 9 in the gas phase (Boltzmann population of 0.86 , see Table S41). The Boltzmann weights of the conformers of $\mathbf{2}, \mathbf{3}, \mathbf{4}, \mathbf{5}, \mathbf{6}$, and 9 change at most by 0.05 in a polar implicit solvent.

All halogen-substituted guests $(7,9,10,11$, and 12) adopt a conformation in the host where the halogens are gauche to each other. The isolated $\left(R^{*}, S^{*}\right)-7,\left(R^{*}, S^{*}\right)-\mathbf{8}$, and $\left(R^{*}, S^{*}\right)-\mathbf{1 0}$ preferentially adopt a conformation in which the halogen groups are placed anti-periplanar to each other (Boltzmann weights of 0.93, 0.95, and 0.99, respectively; see Table S41). The gauche conformation is significantly populated in the gas phase for $\left(R^{*}, R^{*}\right)-\mathbf{7},\left(R^{*}, R^{*}\right)-\mathbf{8}$, and $\left(R^{*}, R^{*}\right)-10$ (Boltzmann weights of $0.65,0.80$, and 0.78 , respectively; see Table S41). The higher association constants of $\left(R^{*}, R^{*}\right)-\mathbf{7},\left(R^{*}, R^{*}\right)-\mathbf{8}$, and $\left(R^{*}, R^{*}\right)-\mathbf{1 0}$ may be partially rationalized by the observation that the gauche conformation, which the guests adopt in the host, is already significantly populated in the gas phase. By contrast, $\left(R^{*}, S^{*}\right)-\mathbf{7},\left(R^{*}, S^{*}\right)-\mathbf{8}$, and $\left(R^{*}, S^{*}\right)$-10 strongly prefer the anti-periplanar conformation in the gas phase and, hence, they likely have to undergo a conformational change when being encapsulated in the host (see also main text).

In a polar solvent, such as acetonitrile, the conformation in which the halogen groups are gauche to each other becomes significantly populated for $\left(R^{*}, S^{*}\right)-\mathbf{7},\left(R^{*}, S^{*}\right)-\mathbf{8}$, and $\left(R^{*}, S^{*}\right)-\mathbf{1 0}$ (Boltzmann weights of $0.27,0.19$, and 0.06 , respectively). Nevertheless, the anti-periplanar conformation is still the preferred one $(0.65,0.74,0.94) .\left(R^{*}, R^{*}\right)-7,\left(R^{*}, R^{*}\right)-\mathbf{8}$, and $\left(R^{*}, R^{*}\right)-\mathbf{1 0}$ generally favor the anti-periplanar conformation in acetonitrile (Boltzmann weights of 0.74 , 0.68 , and 0.57 , respectively).

Replacing the terminal methyl group with a $\mathrm{CF}_{3}$-group in $\left(R^{*}, S^{*}\right)-\mathbf{8}$ and $\left(R^{*}, S^{*}\right)-\mathbf{1 0}$ yields $\left(R^{*}, S^{*}\right)-11$ and $\left(R^{*}, S^{*}\right)-12 .\left(R^{*}, S^{*}\right)-11$ and $\left(R^{*}, S^{*}\right)-12$ preferentially adopt the anti-periplanar conformation in the gas phase (0.83 and 0.97 , see Table S41) and in the polar solution phase ( 0.82 and 0.93 , see Table S41). The population of the other conformations in the gas phase and in the polar solution phase is below 0.16 . 


\section{S3. Effect of Computational Methodology}

The conformational analysis in the Theoretical Section, S2 is based on Boltzmann distributions obtained from electronic energy differences calculated with PBE-D3(BJ) for the PBE-D3(BJ) optimized structures in the gas phase and with PBE-D3(BJ)-CPCM for the PBE-D3(BJ)-CPCM optimized structures in the condensed phase. The application of the implicit solvation model CPCM has a large effect on the electronic energy differences calculated for PBE-D3(BJ)CPCM optimized structures (Figure S130, a). The difference between PBE-D3(BJ)$\mathrm{CPCM} / / \mathrm{PBE}-\mathrm{D} 3(\mathrm{BJ})-\mathrm{CPCM}$ and PBE-D3(BJ)//PBE-D3(BJ)-CPCM energies is on average $0.96 \mathrm{kcal} \mathrm{mol}^{-1}$. The inclusion of electrostatic effects in a polar environment as in CPCM, hence, has a large effect on the predicted Boltzmann distributions. Structures optimized with PBED3(BJ) are similar to the ones optimized with PBE-D3(BJ)-CPCM as evidenced by the fact that DLPNO-CCSD(T) single-point energies evaluated for both structures differ on average difference by only $0.22 \mathrm{kcal} \mathrm{mol}^{-1}$. (Figure S130, b). Furthermore, the DLPNO-CCSD(T)//PBED3(BJ) and DLPNO-CCSD(T)//PBE-D3(BJ)-CPCM energies are strongly correlated (Pearson correlation of 0.93 ). The PBE-D3(BJ) energies deviate on average by $0.31 \mathrm{kcal} \mathrm{mol}^{-1}$ from DLPNO-CCSD(T) energies for PBE-D3(BJ) optimized structures, and the two are strongly correlated with a Pearson correlation of 0.98 (Figure S130, c). We may take this as an indication that the density functional energies are reliable in this case. A calculation of the Boltzmann distributions based on the DLPNO-CCSD(T) energy leads to changes smaller than 0.04 . The consideration of thermal effects calculated within the RRHO model changes the energy differences on average by $0.20 \mathrm{kcal} \mathrm{mol}^{-1}$. Furthermore, the electronic energy differences and the gas-phase free energy differences are strongly correlated ( $R=0.99$, Figure S130, d). 

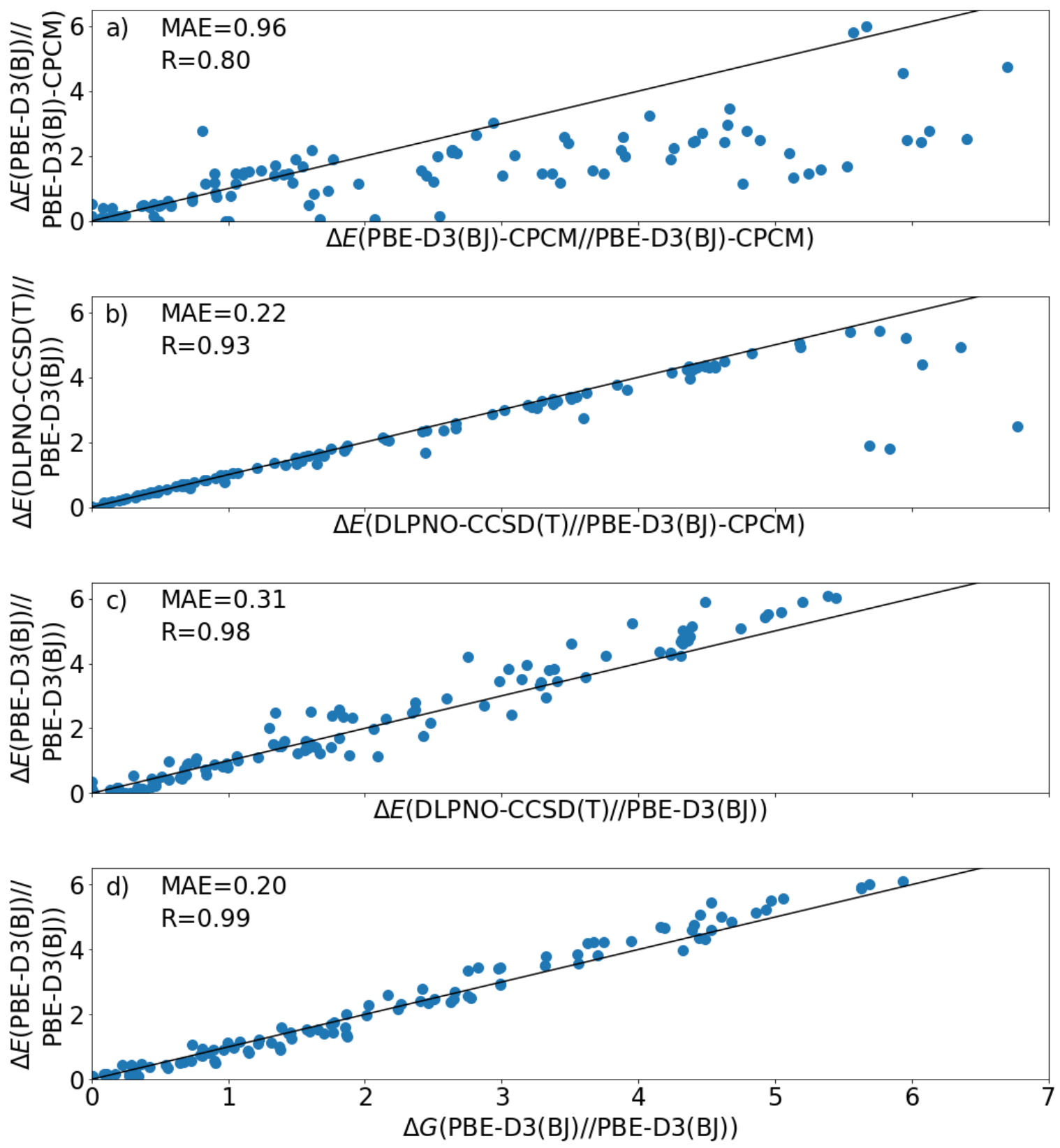

Figure S130. a) Comparison of the electronic energy differences $\Delta E$ obtained with PBE-D3(BJ) and PBE-D3(BJ)CPCM for PBE-D3(BJ)-CPCM optimized structures. b) Comparison of $\triangle E$ obtained with DLPNO-CCSD(T) for PBE-D3(BJ) and for PBE-D3(BJ)-CPCM optimized structures. c) Comparison of $\triangle E$ obtained with DLPNOCCSD(T) and PBE-D3(BJ) for PBE-D3(BJ) optimized structures. d) Comparison of Gibbs free energy differences at $295 \mathrm{~K} \Delta G$ and $\Delta E$ obtained with PBE-D3(BJ) for PBE-D3(BJ) optimized structures. In each panel a-d, we indicate the mean absolute error (MAE) in kcal mol${ }^{-1}$ of the compared data sets and their Pearson correlation (R). All energy differences are given in $\mathrm{kcal} \mathrm{mol}^{-1}$. 


\section{S4. Determination of Packing Coefficients}

We determined the packing coefficients, which are defined as the ratio of the volume of the encapsulated guest molecule to the volume of the cavity formed by the host, for the obtained crystal structures in Tables S42 and S43. The volumes were determined with the program VOIDOO ${ }^{[19]}$ with two different probe sizes as described in our previous work. ${ }^{[7]}$ The obtained cavity volumes (215-234 $\AA^{3}$ and $176-200 \AA^{3}$ for a probe radius of $1.0 \AA$ and of $1.6 \AA$, respectively) are similar to the ones obtained in our previous work ${ }^{[7]}\left(190-242 \AA^{3}\right.$ and 154 $191 \AA^{3}$ for a probe radius of $1.0 \AA$ and of $1.4 \AA$, respectively). This leads to packing coefficients of $41-53 \%$ for a probe radius of $1.0 \AA$ and of $48-61 \%$ for a probe radius of $1.6 \AA$ (see also Tables S42 and S43). In this case, a probe radius of $1.6 \AA$ leads to in better agreement with the Mecozzi-Rebek volume occupancy rule of $55 \%$ than a probe radius of $1.0 \AA .{ }^{[20 \mathrm{a}]}$ For molecular baskets encapsulating heteroalicyclic guests undergoing polar interactions, the volume occupancies can increase to $63 \%$. ${ }^{[20 b]}$

Table S42. Guest volumes in $\AA^{3}$, cavity volumes in $\AA^{3}$ with a probe size of $1.0 \AA$ and $1.6 \AA$, and packing coefficients in percent for co-crystal structures of AAC $(P)_{4}-\mathbf{1}$.

\begin{tabular}{|l|l|l|l|l|l|}
\hline \multicolumn{1}{|c|}{$\begin{array}{c}\text { Encapsulated } \\
\text { guest }\end{array}$} & $\begin{array}{c}\text { Guest } \\
\text { volume } \\
{\left[\AA^{3}\right]}\end{array}$ & $\begin{array}{c}\text { Cavity } \\
\text { volume } \\
{\left[\AA^{3}\right] \text { with }} \\
\text { probe size } \\
\text { of } 1.0 \AA\end{array}$ & $\begin{array}{c}\text { Packing } \\
\text { coefficient }[\%] \\
\text { with probe size } \\
\text { of } 1.0 \AA\end{array}$ & $\begin{array}{c}\text { Cavity } \\
\text { volume } \\
{\left[\AA^{3}\right] \text { with }} \\
\text { probe size } \\
\text { of } 1.6 \AA\end{array}$ & $\begin{array}{c}\text { Packing } \\
\text { coefficient } \\
{[\%] \text { with }} \\
\text { probe size of } \\
1.6 \AA\end{array}$ \\
\hline $\mathbf{5}$ & 92 & 223 & 41 & 188 & 48 \\
\hline$\left(R^{*}, S^{*}\right)-\mathbf{7}$ & 98 & 223 & 43 & 185 & 53 \\
\hline$\left(R^{*}, R^{*}\right)-\mathbf{7}$ & 100 & 215 & 46 & 176 & 56 \\
\hline$\left(R^{*}, S^{*}\right)-\mathbf{8}$ & 109 & 229 & 47 & 196 & 55 \\
\hline$\left(R^{*}, R^{*}\right)-\mathbf{8}$ & 109 & 224 & 48 & 188 & 57 \\
\hline $\mathbf{9}$ & 104 & 234 & 44 & 200 & 52 \\
\hline$\left(R^{*}, S^{*}\right)-\mathbf{1 0}$ & 121 & 229 & 52 & 197 & 61 \\
\hline$\left(R^{*}, R^{*}\right)-\mathbf{1 0}$ & 120 & 232 & 51 & 200 & 60 \\
\hline
\end{tabular}


Table S43. Guest volumes in $\AA^{3}$, cavity volumes in $\AA^{3}$ with a probe size of $1.0 \AA$ and $1.6 \AA$, and packing coefficients in percent for co-crystal structures of AAC $(M)_{4}-\mathbf{1}$.

\begin{tabular}{|l|l|l|l|l|l|}
\hline \multicolumn{1}{|c|}{\begin{tabular}{|} 
Encapsulated \\
guest
\end{tabular}} & $\begin{array}{c}\text { Guest } \\
\text { volume } \\
\left(\AA^{3}\right)\end{array}$ & $\begin{array}{c}\text { Cavity } \\
\text { volume } \\
\left(\AA^{3}\right) \\
\text { Probe size } \\
\text { of } 1.0 \AA\end{array}$ & $\begin{array}{c}\text { Packing } \\
\text { coefficient [\%] } \\
\text { Probe size of } \\
1.0 \AA\end{array}$ & $\begin{array}{c}\text { Cavity } \\
\text { volume } \\
\left(\AA^{3}\right) \\
\text { Probe size } \\
\text { of } 1.6 \AA\end{array}$ & $\begin{array}{c}\text { Packing } \\
\text { coefficient } \\
{[\%] \text { Probe }} \\
\text { size of } 1.6 \AA\end{array}$ \\
\hline$\left(S^{*}, R^{*}\right)-\mathbf{7}$ & 99 & 226 & 43 & 184 & 53 \\
\hline$\left(S^{*}, S^{*}\right)-\mathbf{7}$ & 97 & 226 & 42 & 187 & 52 \\
\hline$\left(S^{*}, R^{*}\right)-\mathbf{8}$ & 108 & 227 & 47 & 177 & 60 \\
\hline$\left(S^{*}, S^{*}\right)-\mathbf{8}$ & 109 & 222 & 48 & 183 & 59 \\
\hline $\mathbf{9}$ & 104 & 234 & 44 & 200 & 52 \\
\hline$\left(S^{*}, R^{*}\right)-\mathbf{1 0}$ & 122 & 230 & 53 & 193 & 62 \\
\hline$\left(S^{*}, S^{*}\right)-\mathbf{1 0}$ & 120 & 233 & 51 & 198 & 60 \\
\hline
\end{tabular}

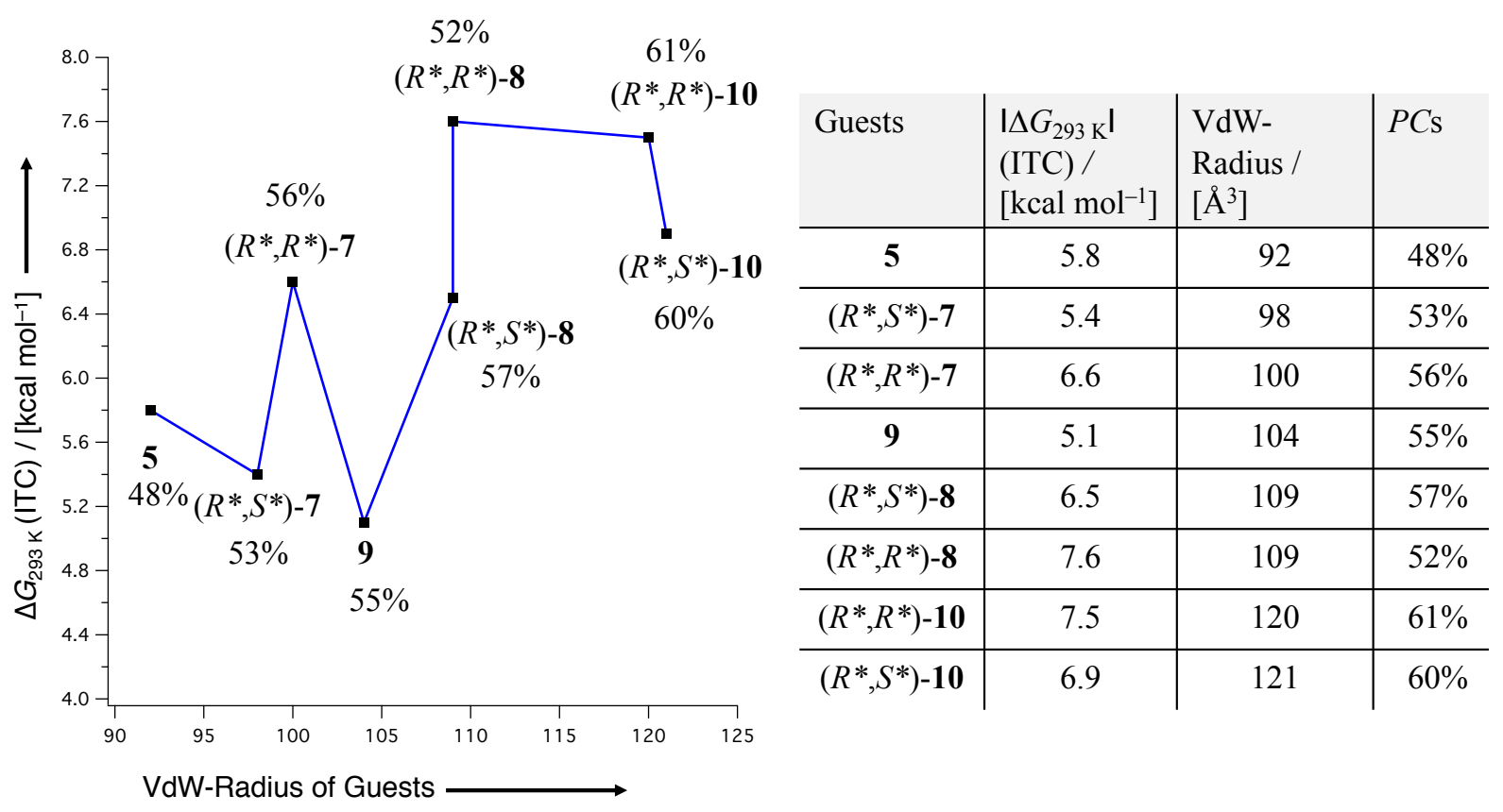

Figure S131. Plot of the experimental values of the association constants of the guests in $\mid \Delta G_{293 \mathrm{~K}} \mathrm{I}$ (ITC) in kcal $\mathrm{mol}^{-1}$ against the Van-der-Waals-Radii of the guests as determined in Section S4 of the Theoretical Section. The packing coefficients $\left(P C, P C=V_{\mathrm{VdW}}(\mathrm{Guest}) / V_{\mathrm{VdW}}(\right.$ Host $\left.) \times 100 \%\right)$, see Section $\mathrm{S} 4$ of the Theoretical Section. All $P C$-calculations are based on obtained $\mathrm{X}$-ray co-crystal structures. 


\section{References}

[1] Gropp, C.; Trapp, N.; Diederich, F. Alleno-Acetylenic Cage (AAC) Receptors: Chiroptical Switching and Enantioselective Complexation of trans-1,2Dimethylcyclohexane in a Diaxial Conformation. Angew. Chem. Int. Ed. 2016, 55, 14444-14449.

[2] MicroCal Origin, Version 5.0, Northampton, USA, 1998.

[3] CrysAlisPro, Rigaku Oxford Diffraction, 2015-2017.

[4] Dolomanov, O. V.; Bourhis, L. J.; Gildea, R. J.; Howard, J. A. K.; Puschmann, H. OLEX2: A Complete Structure Solution, Refinement and Analysis Program. J. Appl. Cryst. 2009, 42, 339-341.

[5] G. M. Sheldrick, Acta Cryst. A 2008, 64, 112-122.

[6] IgorPro, Wavemetrics, Version 6.37, Portland, USA, 2009.

[7] Gropp, C.; Husch, T.; Trapp, N.; Reiher, M.; Diederich, F. Dispersion and HalogenBonding Interactions: Binding of the Axial Conformers of Monohalo- and ( \pm )-trans1,2-Dihalocyclohexanes in Enantiopure Alleno-Acetylenic Cages. J. Am. Chem. Soc. 2017, 139, 12190-12200.

[8] Gropp, C.; Husch, T.; Trapp, N.; Reiher, M.; Diederich, F. Hydrogen-Bonded Networks: Molecular Recognition of Cyclic Alcohols in Enantiopure Alleno-Acetylenic Cage Receptors. Angew. Chem. Int. Ed. 2018, 57, 16296-16301.

[9] Koźmiński, W.; Nanz, D. HECADE: HMQC- and HSQC-Based 2D NMR Experiments for Accurate and Sensitive Determination of Heteronuclear Coupling Constants from E.COSY-Type Cross Peaks. J. Magn. Res. 1997, 124, 383-392.

[10] O’Boyle, N. M.; Vandermeersch, T.; Flynn, C. J.; Maguire, A. R.; Hutchison, G. R. Confab - Systematic Generation of Diverse Low-energy Conformers. J. Cheminform. 2011, 3, 8 .

[11] O’Boyle, N. M.; Banck, M.; James, C. A.; Morley, C.; Vandermeersch, T.; Hutchison, G. R Open Babel: An Open Chemical Toolbox. J. Cheminform. 2011, 3, 33.

[12] Neese F. WIREs Comput. Mol. Sci. 2018, 8, e1327.

[13] a) Perdew, J. P.; Wang, Y. Accurate and Simple Analytic Representation of the Electron-gas Correlation Energy. Phys. Rev. B 1992, 45, 13244-13249; b) Perdew, J. P.; Burke, K.; Ernzerhof, M. Generalized Gradient Approximation Made Simple. Phys. Rev. Lett. 1996, 77, 3865-3868.

[14] Grimme, S.; Antony, J.; Ehrlich, S.; Krieg, H. A Consistent and Accurate Ab Initio Parametrization of Density Functional Dispersion Correction (DFT-D) for the 94 Elements H-Pu. J. Chem. Phys. 2010, 132, 154104. 
[15] Weigend, F.; Ahlrichs, R. Balanced Basis Sets of Split Valence, Triple Zeta Valence and Quadruple Zeta Valence Quality for H to Rn: Design and Assessment of Accuracy. Phys. Chem. Chem. Phys. 2005, 7, 3297-3305.

[16] Klamt, A.; Schüürmann, G. COSMO: A New Approach to Dielectric Screening in Solvents with Explicit Expressions for the Screening Energy and its Gradient. J. Chem. Soc., Perkin Trans. 2, 1993, 799-805.

[17] McQuarrie, D. A. Statistical Mechanics; University Science Books: Sausalito, 2000.

[18] Riplinger, C.; Neese, F. An Efficient and Near Scaling Pair Natural Orbital Based Local Coupled Cluster Method. J. Chem. Phys. 2013, 138, 034106.

[19] Kleywegt, G. J. VOIDOO, version 3.3.4, Uppsala Software Factory, Uppsala, 2007.

[20] a) Mecozzi, S.; Rebek, J. Jr. The $55 \%$ Solution: A Formula for Molecular Recognition in the Liquid State. Chem. Eur. J. 1998, 4, 1016-1022; b) Hornung, J.; Fankhauser, D.; Shirtcliff, L. D.; Praetorius, A.; Schweizer, W. B.; Diederich, F. Cycloalkane and Alicyclic Heterocycle Complexation by New Switchable Resorcin[4]arene-based Container Molecules: NMR and ITC Binding Studies. Chem.-Eur. J. 2011, 17, 12362 12371. 